\title{
Exploring a unique vision for heavy ion fusion:
}

B. Grant Logan, August 6, 2007 Fifth Edition:study of ion two-sided polar direct drive of T-lean targets.

A quest for more efficient beam-to-fuel energy coupling via polar direct drive (30\% overall), to enable:

--> Self-T-breeding, self-neutron-energy-absorbing, large $\rho r$, T-Lean targets @ $<4$ MJ driver energies

--> Efficient fusion energy coupling into plasma for direct MHD conversion with moderate yields < $1 \mathrm{GJ}$

--> Balance-of-plant costs 10X lower than steam cycle (e.g., $<80 \$ / \mathrm{kWe}$ instead of $800 \$ / \mathrm{kWe}$ )

--> CoE low enough ( $<3 \mathrm{cts} / \mathrm{kWehr}$ ) for affordable water and $\mathrm{H}_{2}$ fuel for $10 \mathrm{~B}$ people on a hot planet.

--> Enough fissile fuel production for 38 LWR's per $\mathrm{GW}_{\text {fusion }}$ if uranium gets too expensive meantime.

\section{TABLE OF CONTENTS}

Title page and Table of Contents

Page 1

Introduction-what we are seeking and why

Comparing direct and indirect-drive coupling efficiency for T-lean targets

Why plasma MHD conversion is synergistic with large rho-r T-lean targets

Compact Fusion Advanced Rankine Cycle description

MathCAD Model for T-lean fuel capsule burn parameters

Comparison of model fuel gain vs Tabak burn calculations

Model for net T production in T-lean fuel assemblies

Comparison of Atzeni and Tabak T-lean burn calculations

CFAR direct conversion scheme applied to polar drive of T-Lean targets

Neutron attenuation factors

Fraction of yield captured in targets and target shells

Rocket efficiency with pure $\mathrm{H} 2$ ablators

Radiation losses, opacity and preheat avoidance

Pie-sector diagram for Power plant T-lean fuel capsule

Pie-sector diagram for DEMO T-lean fuel capsule

Summary of model fuel stagnation assemblies and burn parameters

Beam stopping range vs ion energy for various ions-HYDRA model

lon $\mathrm{dE} / \mathrm{dx}$ profiles for Argon and Krypton beams

Bragg peak effect enhancing beam delived to ablation front

Implosion dynamics for Case A Power Plant $E_{f}=1 M J, M_{o} / M_{f}=5$

Estimate of late spike method of reducing parasitic beam losses

Estimate local reduction of ablated column density for two-sided drive

Beam requirments for Case $A$ Power Plant $E_{f}=1 M J, M_{o} / M_{f}=5$

Page 2

Page 3

Page 4

Page 5

Page 6

Page 7

Page 8

Page 9

Page 11

Page 12

Page 13

Page 14

Page 15

Page 16

Page 17

Page 18

Page 19

Page 22

Page 23

Page 24

Page 28

Beam requirements for Case $B$ DEMO $E_{f}=0.2 \mathrm{MJ}, M_{o} / M_{f}=5$

Page 28

Page 30

Page 35

Beam requirements for Case $C$ Power Plant $E_{f}=1 M J, M_{o} / M_{f}=2$

Page 40

Rough power plant parameters and cost model

Summary for power plant and DEMO cases

2-D time-dependent polar (two-sided) beam requirements

Beam requirements for Case $A$ implosions with polar beam illumination

Summary of 2-D Beam requirements for large T-lean reactor Case A

Beam requirements for Case $B$ implosions with polar beam illumination

Summary of 2-D Beam Requirments for small DEMO Case B

Page 41

Page 43

Page 44

Page 47

Page 61

Page 62

Page 75

Comparison of range lengthening for DEMO cases with $M_{o} / M_{f}=5 \& M_{o} / M_{f}=2$

Estimating incident beam perveance, assuming no plasma neutralization

Page 78

Page 79

Summary comments

Page 80 


\section{Introduction- what we are seeking and why}

In 1996 and 1997 two studies of tritium-lean targets [1], [2] showed that large fuel assemblies $\left(\rho r_{\mathrm{f}} \sim 10 \mathrm{~g} / \mathrm{cm}^{2}\right)$ with about $1 \%$ molar tritium fraction concentrated in a small DT spark plug surrounded by deuterium could achieve fuel energy gains of 500 and 1000 with isobaric [1] and isochoric (fast ignited) [2] hot-spot ignition, respectively, in both cases with sufficient net $T$ breeding by $D(d, n) T$ side reactions to avoid the need for any external $T$-breeding blankets. Those studies also noted the possibility of such targets providing more extra neutrons that could be used for additional breeding in external blankets, such as for fissile fuel production, and noted larger fractions of fusion yield in charged particles for direct conversion, compared to all-DT targets. A major drawback was the large fuel masses $>20$ mg and fuel energies $\mathrm{E}_{\mathrm{f}}$ required at stagnation $\sim 1 \mathrm{MJ} @$ adiabat $\alpha=2$ and 1.5, respectively, which implied very large required driver energies ( $>10 \mathrm{MJ}$ for $=$ laser direct drive efficiency (beam-to-fuel) $\eta_{\mathbf{d f}}<0.1$, and $>17 \mathrm{MJ}$ for indirect drive with $\eta_{d f}<0.06$ ( 0.3 hohlraum coupling x 0.2 x-ray capsule implosion efficiency). Here we seek $\eta_{d f}>0.25$ with ion direct drive.

This MathCAD document presents a numerical implosion model for T-Lean targets driven in the ablative direct drive regime using the same heavy-ion $\mathrm{dE} / \mathrm{dx}$ deposition model as used in the LLNL HYDRA code. This model explores characteristic beam requirements for such targets, as a guide to motivate hydro code calculations for 2-D polar drive. Thick hydrogen ablators (e.g., $92 \mathrm{mg}$ initial mass) are divided into 30 Lagrangian mass layers, and the model specifies ion ablation of those layers at a rate to achieve a specified drive pulse shape at the peak of rocket efficiency $(80 \% \mathrm{mass}$ ablation fraction by the time the ablation front radius $=50 \%$ of initial ablator radius). Ion beams stop efficiently in hydrogen ablators (most electrons per unit mass) which also have lowest specific ionization energy $<<u^{2}{ }_{\text {ex }} / 2 . H i g h$ ablation velocities in these thick ablator rocket regimes lead to improved stability as well as lower exhaust velocities $u_{e x}=2 c_{s}<1.910^{7} \mathrm{~cm} / \mathrm{s}$ that imply low stagnation $T_{e}<30 \mathrm{eV}$ and low radiation losses. However, ion beams can suffer greater parasitic energy loss passing through ablation corona plasmas compared to laser or $x$-ray drive photons, despite the $\mathrm{dE} / \mathrm{dx}$ Bragg peak near the end of the ion range.

An initial ion beam range $0.004 \mathrm{~g} / \mathrm{cm} 2$ is selected to be $20 \%$ of the initial hydrogen ablator $\rho r_{h o}=0.02 \mathrm{~g} / \mathrm{cm} 2$. If the ion energy were constant, the ions would stop short of the ablation front early into the drive pulse due to rising ablation plasma column density which the model tracks during the drive pulse. Working backwards, the model calculates the higher incident ion beam energy and losses at each time step for ions required to penetrate the rising ablation plasma column density and then stop in the remaining hydrogen ablator shell. An example for a 1 MJ T-lean final fuel assembly finds required incident Argon ion beam energy rising from 250 to $750 \mathrm{MeV}$ during the pulse (which is actually synergistic with neutralized beam compression and focusing with high compression velocity chirp and time dependent focusing). A total incident ion beam energy of $4 \mathrm{MJ}$ is required (25\% overall drive efficiency assuming zooming), despite $40 \%$ loss of ion energy in the ablation corona. Ways to further mitigate the ion beam loss on ablation plasma are discussed for future two-sided (polar) direct drive. Examples for both a small 1 MJ drive DEMO as well as for a 4 MJ drive power plant with 50\%-efficient plasma-shell MHD direct conversion [3] are summarized in the table just below and compared with earlier indirect drive heavy ion target designs for DT targets. A summary at the end of this document gives more details for the two selected T-lean IFE examples.

[1] TABAK, M. Nuclear Fusion 36, No 2 (1996)

[2] ATZENI, S., and CIAMPI, C., Nuclear Fusion 37, 1665 (1997)

[3] LOGAN, B. G., Fusion Engineering and Design 22, 151 (1993)

[4] ATZEHI, S. and MEYER-TER-VEHN, J., "The Physics of Inertial Fusion" Clarendon press-Oxford, 2004 


\section{T-lean fuel assemblies for MHD direct conversion Develop and benchmark MCAD}

model based on Tabak's Case $C$ run 1 for isobaric hot spot ignition (see Fig. 1) with his $f=0.1$ tritium parameter (to maximize non-neutron yield for direct conversion with $\eta G_{f}>100$ for low recirculating power )

\section{TABAK}

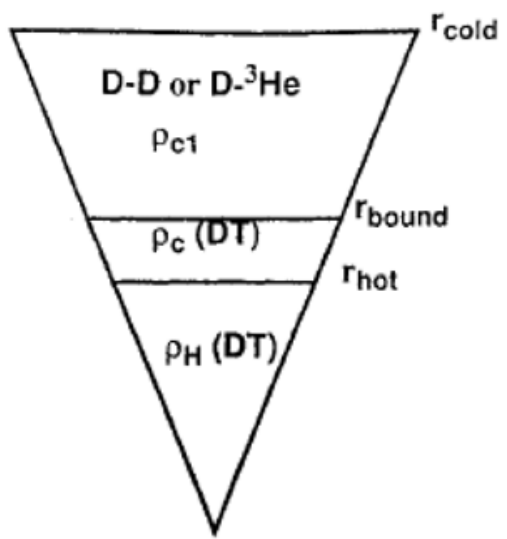

FIG. 1. Pie diagram of compressed core.
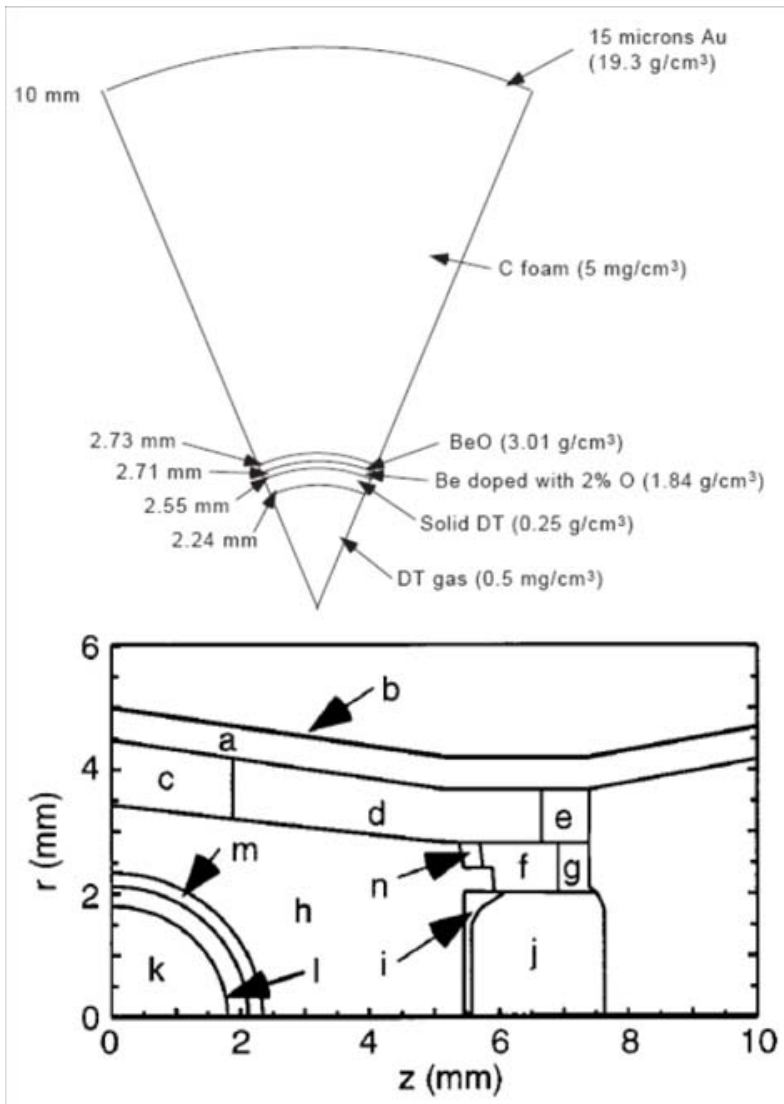

Ref [1] Calculates DD burn assuming $10 \mathrm{keV}$ initial DT hot spot. (If needed, a late shock (Betti- Perkins) could be applied to insure ignition at lower implosion velocities and at lower adiabats, say $\alpha=1.5$ instead of at $\alpha=2$ as Max assumed). We assume DD main fuel instead of D-3He for reasons Max stated.

Ref [1] calculates burn starting with stagnation with a range fuel energies, both below and above $1 \mathrm{MJ}$. The compressed core shown in Fig. 1 is representing the fuel assembly at stagnation after whatever ablator has burned off. Lets compare a few examples we will be developing for direct drive with previous heavy ion indirect drive targets, to motivate seeking higher beam-to-fuel coupling efficiencies to enable such T-lean targets.
The spherical illumination indirect drive lithium hohlraum target designed by Allshouse and Callahan (Nuclear Fusion, Vol. 39, No.7 (1999). The large case-to-capsule ratio was necessary to provide adequate implosion symmetry by both thermal and geometrical smoothing of $x$-rays from beam deposition non-uniformities. $16 \mathrm{MJ}$ of total lithium ion drive, with a $100 \mathrm{TW}, 40 \mathrm{~ns}$ foot pulse provided by 32 beams @ 17 to 22 $\mathrm{MeV}$, followed by a $600 \mathrm{TW}, 15 \mathrm{~ns}$ main pulse provided by 12 beams. (other parameters are in the table below)

A diagram of $1 / 4$ of the capsule and hohlraum for the close-coupled target by Callahan \& Tabak (Physics of Plasmas, Vol. 6, No 4, May 2000). This design had a smaller hohlraum for the same capsule size as a previous distributed radiator target (DRT) used for the Robust Point Design. (Callahan and Tabak, Physics of Plasmas, 5, 1895 (1998). See table below. The materials and densities used were as follows: (a) AuGd at $0.1 \mathrm{~g} / \mathrm{cc}$, (b) 15 microns layer of AuGd at $13.5 \mathrm{~g} / \mathrm{cc}$, (c) Fe at $16 \mathrm{mg} / \mathrm{cc}$, (d)(CD2)0.97Au0.03 at $11 \mathrm{mg} / \mathrm{cc}$, (e) AuGd at $0.11 \mathrm{~g} / \mathrm{cc}$, (f) Al at $70 \mathrm{mg} / \mathrm{cc}$, (g) AuGd at $0.26 \mathrm{~g} / \mathrm{cc}$, (h) $\mathrm{CD} 2$ at $1 \mathrm{mg} / \mathrm{cc}$, (i) $\mathrm{Al}$ at $55 \mathrm{mg} / \mathrm{cc}$, (j) AuGd sandwich with densities $0.1 \mathrm{~g} / \mathrm{cc}$, $1.0 \mathrm{~g} / \mathrm{cc}$, and $0.5 \mathrm{~g} / \mathrm{cc},(\mathrm{k}) \mathrm{DT}$ at $0.3 \mathrm{mg} / \mathrm{cc}$, (1) DT at $0.25 \mathrm{~g} / \mathrm{cc}$, (m) Be0.995Br0.005 at $1.845 \mathrm{~g} / \mathrm{cc}$, (n) (CD2) $0.97 \mathrm{Au} 0.03$ at $32 \mathrm{mg} / \mathrm{cc}$.

Fig. 2. Previous heavy ion indirect-drive target designs (published). 


\begin{tabular}{|l|l|l|l|l|l|l|l|l|l|l|l|l|}
\hline & $\begin{array}{l}\mathrm{E}_{\mathrm{d}} \\
\mathrm{MJ}\end{array}$ & $\begin{array}{l}\mathrm{T}_{\text {rad }} \\
(\mathrm{eV})\end{array}$ & $\begin{array}{l}\mathrm{E}_{\text {cap }} \\
(\mathrm{kJ})\end{array}$ & $\begin{array}{l}\mathrm{R}_{\mathrm{ho}} \\
(\mathrm{mm})\end{array}$ & $\begin{array}{l}\mathrm{A}_{\text {wall }} \\
\left(\mathrm{cm}^{2}\right)\end{array}$ & $\begin{array}{l}\mathrm{R}_{\text {cap }} \\
(\mathrm{mm})\end{array}$ & $\begin{array}{l}\mathrm{E}_{\text {fuel }} \\
(\mathrm{kJ})\end{array}$ & $\begin{array}{l}\text { Yield } \\
(\mathrm{MJ})\end{array}$ & $\begin{array}{l}\text { Target } \\
\text { gain }\end{array}$ & $\begin{array}{l}\mathrm{M}_{\text {conv }} \\
(\mathrm{mg})\end{array}$ & $\begin{array}{l}\eta_{\text {df }} \\
(\%)\end{array}$ & $\begin{array}{l}\mathrm{I}_{\text {b-peak }} \\
\text { W/cm }\end{array}$ \\
\hline $\begin{array}{l}\text { SNL Li } \\
\text { hohlraum }\end{array}$ & 16 & 220 & 1400 & 10 & 12.6 & 2.7 & 280 & 591 & 37 & 250 & 1.8 & $4.8 \mathrm{e} 13$ \\
\hline $\begin{array}{l}\text { DRT-Robust } \\
\text { point design }\end{array}$ & 6.7 & 245 & 1000 & 5.4 & 5.0 & 2.3 & 200 & 436 & 66 & 80 & 3.0 & $3.3 \mathrm{e} 14$ \\
\hline $\begin{array}{l}\text { DRT Close - } \\
\text { coupled }\end{array}$ & 3.3 & 245 & 1000 & 4.0 & 2.7 & 2.3 & 200 & 436 & 133 & 24 & 6.0 & $6.2 \mathrm{e} 14$ \\
\hline $\begin{array}{l}2.5 \text { MJ HI } \\
\text { Cannonball }\end{array}$ & 2.5 & 300 & 800 & 3.0 & 1.1 & 1.8 & 160 & 300 & 120 & 40 & 6.4 & $6 \mathrm{e} 14$ \\
\hline $\begin{array}{l}1.5 \text { MJ HI } \\
\text { Cannonball }\end{array}$ & 1.5 & --- & 530 & 2.4 & 0.72 & 1.4 & 100 & 150 & 100 & 25 & 6.7 & $8 \mathrm{e} 14$ \\
\hline NIF (laser) & 1.8 & 300 & 175 & 2.5 & 1.4 & 1 & 35 & 20 & 11 & na & 1.9 & $1 \mathrm{e} 15$ leh \\
\hline $\begin{array}{l}\text { HI-dd-direct } \\
\text { drive (1.2 GWe) }\end{array}$ & 4 & na & $\begin{array}{l}2550 \\
\text { ablator }\end{array}$ & na & na & 7.1 & 1000 & $\begin{array}{l}494 \\
(\text { Tlean })\end{array}$ & 123 & na & 25 & $2.1 \mathrm{e} 14$ \\
\hline $\begin{array}{l}\text { HI-dd-direct } \\
\text { drive DEMO }\end{array}$ & 0.8 & na & $\begin{array}{l}490 \\
\text { ablator }\end{array}$ & na & na & 3.7 & 200 & $\begin{array}{l}43 \\
(\text { Tlean })\end{array}$ & 52 & na & 24 & $3.1 \mathrm{e} 14$ \\
\hline
\end{tabular}

Table 1: The two entries referred to as "Cannonballs" are rough constructs of close-coupled spherical hohlraums using scalings from Lindl's book, not necessarily representing any previous designs. Symmetry is addressed in the three published indirect drive designs above, but symmetry in the close-coupled cannonball examples may be a challenge due to small case-to-capsule ratios. All close-coupled hohlraum examples, cylindrical or spherical, can get beam-to-fuel coupling efficiencies $\eta_{\mathrm{df}} \sim 6$ to $7 \%$ with DT gains $>100$ and higher peak intensity beams incident on

target $>600 \mathrm{TW} / \mathrm{cm}^{2}$ (average of overlapping incident beams $-10 \mathrm{x}$ higher than for the SNL Li hohlraum design). The last two rows are numerical calculations for heavy ion ablative direct drive cases for T-lean targets with $\sim 25 \%$ coupling (this work). Note that with lower overall coupling efficiencies, indirect drive would require higher driver energies and associated peak beam intensities to create the same fuel energies for the two T-lean cases. $\underline{T h e}$ important thing is that heavy ions with the right range can in principle achieve similar ablation velocities, stabilitv, and rocket efficiency with thick ablators as do $x$-ravs without having to incur the inefficiency of conversion to x-ravs. The analysis below finds that ion beams may suffer more parasitic losses on the ablation corona than $x$-rays, but that is compensated by using hydrogen ablators that have less ionization energy losses than $x$-ray ablators require for to be optically thick using atomic $Z>4$ to 6 for the hohlraum $x$-ray temperatures. The incident ion beam smoothness and symmetry will be required as much as with laser direct drive, including in polar direct drive geometry, and we will be investigating techniques to achieve required smoothness and symmetry by twirling arrays of ion beams around the polar axis using upstream R.F. wobblers -those topics will be addressed in a subsequent MathCAD model extension.

Why plasma MHD conversion is synergistic with large $\rho$ r T-lean targets.

Before beginning the description of the MathCAD model for ion direct drive, lets digress briefly on why we seek forms of fusion energy production into $1 \mathrm{-2} \mathrm{eV}$ plasmas for MHD conversion, and why the consideration now of $\underline{T-l e a n}$ targets offers a solution to enable efficient capture of fusion yield into that desired form at reasonable fusion yields $<1$ GJ. Ref. [3] above found that direct MHD conversion could be most efficient (greater than 50\%) for dense (10 to 100 bar) plasmas containing an alkali component such as lithium or potassium with optimal temperatures of 1 to $2 \mathrm{eV}$. Below that optimum temperature, the plasma conductivity decreases strongly with the ionization fraction, and above those temperatures, plasma radiation losses decrease the convertible plasma energy. Following the original idea proposed by Velikhov, the Compact Fusion Advanced Rankine cycle study [3] assumed a solid target shell of chosen working material around each target, where the shell had to be thick enough to stop $14 \mathrm{MeV}$ DT neutrons, and where the fusion yield had to be sufficient (typically several 10's of GJ) to vaporize and ionize the shell mass (typically 100 's of $\mathbf{~ k g}$ ) into an average 1-2 eV plasma temperature for optimal MHD conversion. Those earlier studies neglected the neutron energy losses within the target $\rho$, (typically 10 to $20 \%$ for DT targets), and the resulting required large target shell masses (to capture most of the $14 \mathrm{MeV}$ neutron energy) forced the requirement of very large fusion yields (10's of GJ) that discouraged further pursuit of the concept. Now, the possibility of creating T-lean fuel assemblies with large $\rho r \sim 10 \mathrm{~g} / \mathrm{cm}^{2}>2 \mathrm{x}$-average neutron mean-free-paths, and doing so with higher beam-to-fuel coupling efficiencies to keep the driver energies reasonable, opens the possibility of efficient capture of T-lean target fusion yields a hundred times smaller than in the previous $C F A R$ study, allowing correspondingly smaller fusion yields for efficient MHD conversion. Fig. 3 below provides basic information on plasma MHD conversion for those who may not be familiar with it. 
Momentum :

$\rho u \frac{d u}{d x}+\nabla p=j x B-\rho u^{2} \frac{d f}{d x}$

Energy :

$\rho u \frac{d}{d x}\left[\frac{u^{2}}{2}+h\right]=j \cdot E-q r$

Continuity :

ou $\mathbf{A}=$ constant
Load Factor :

$K=\frac{E}{u B}$

Electric Power Density :

$$
\mathrm{j} \cdot \mathrm{E}=-\frac{\mathrm{K}(1-\mathrm{K}) \sigma \mathrm{u}^{2} \mathrm{~B}^{2}}{1+\left(\omega \tau^{2}\right)}
$$

Magnet cost, Energy Density :

Magnet Cost $/ \mathrm{m}^{3} \sim \mathrm{B}^{2} /\left(2 \mu_{o}\right)$

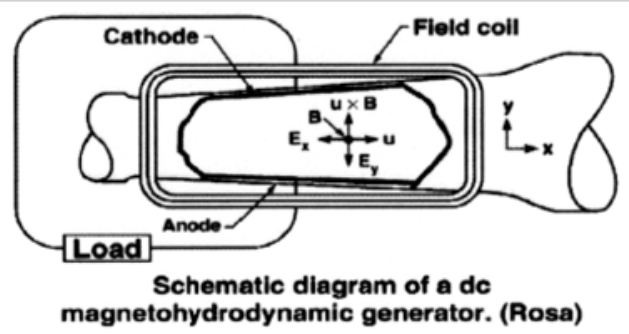

magnetohydrodynamic generator. (Rosa)

where $\rho=$ mass density, $\mathrm{u}=$ velocity, $\mathrm{x}=$ distance along channel, $\mathrm{p}=$ pressure, $j=$ current density, $B=$ magnetic field, $E=$ electric field, $\sigma=$ electrical conductivity, $\omega \tau=$ Hall parameter, $h=$ specific enthalpy, $f=$ friction due to mass inflow from wall transpiration cooling, $q r=$ heat loss $/ \mathrm{m}^{3}$ due to radiation losses, $A=$ channel area.

$\rightarrow$ MHD Magnet Cost / kWe $\sim \mathrm{B}^{2} / \mathrm{j} \cdot \mathrm{E} \quad \rightarrow$ Figure-of-merit $\$ / \mathrm{kWe} \sim\left(\sigma \mathrm{u}^{2}\right)^{-1}$
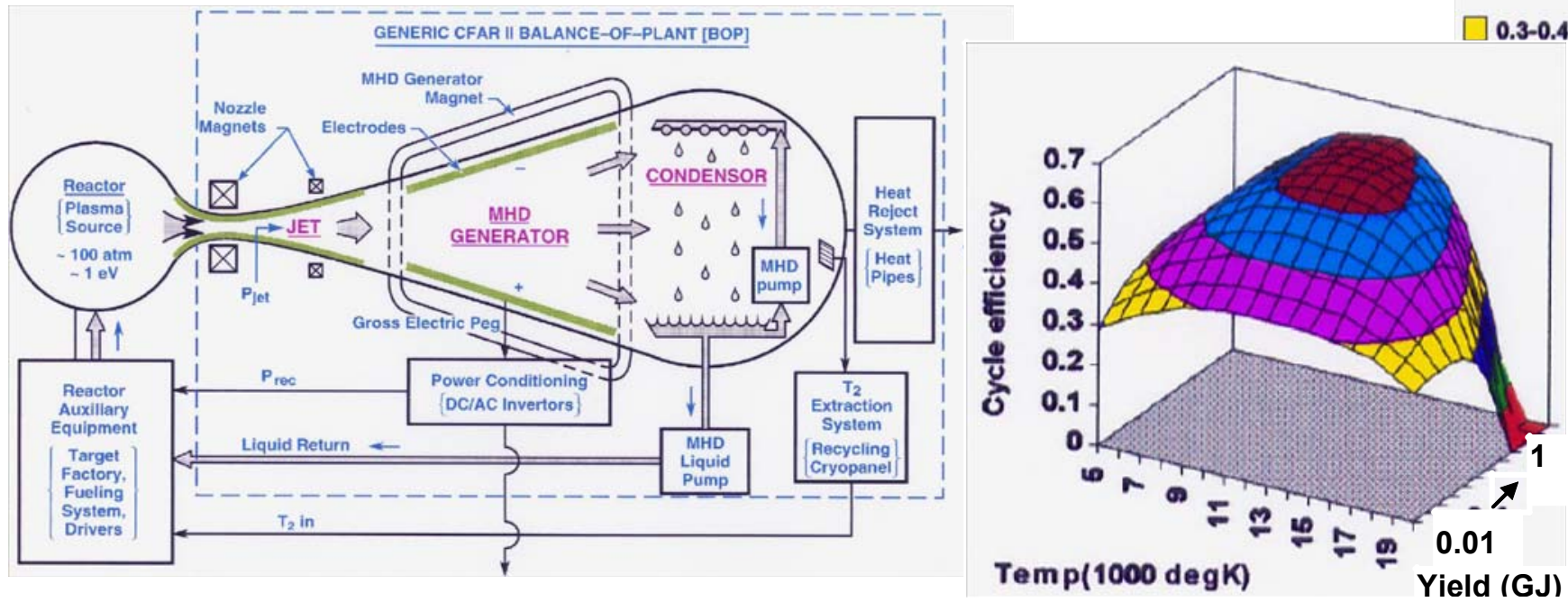

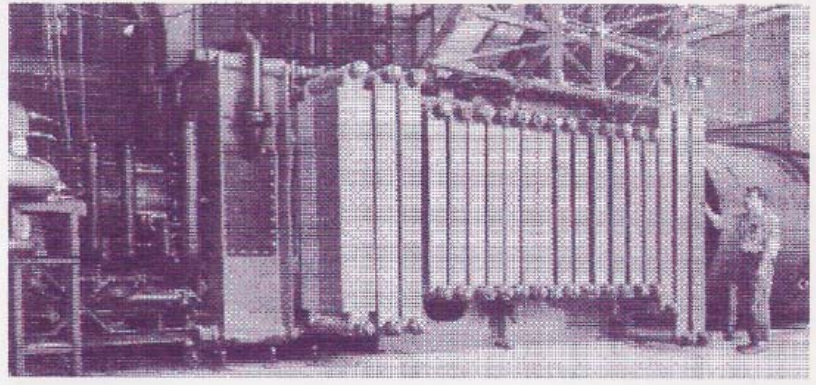

THE AVCO MARK V "ROCKET GENERATOR" ( from Rosa, MHD Energy Conversion, 1968)

Temp $\sim 3000$ deg $\mathrm{K}$

Conductivity $\sim 100 \mathrm{mhos} / \mathrm{m}$

Velocity $\sim 1000 \mathrm{~m} / \mathrm{s}$

Efficiency $\sim 10 \%$ (of chemical)

Power ( above unit) 20 MWe
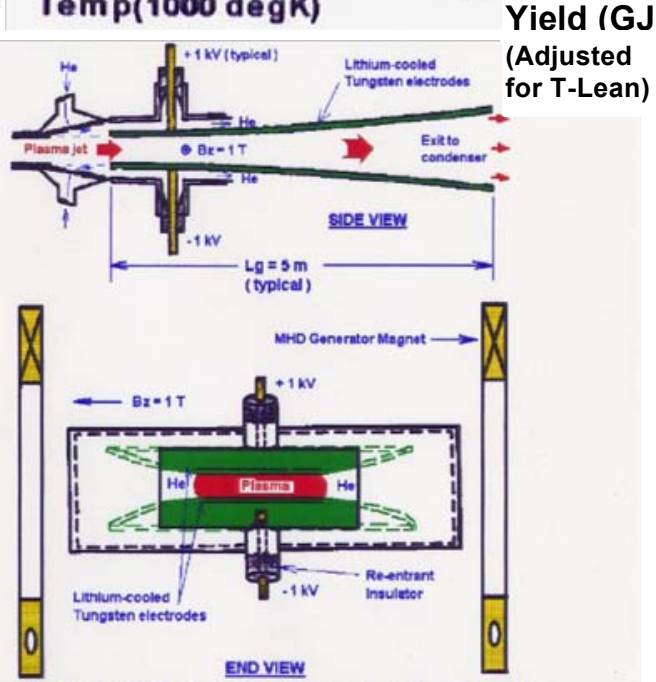

TYPICAL" CFARII MHD GENERATOR

Temp $\sim 12000 \mathrm{deg} \mathrm{K}$, Conductivity $\sim 500 \mathrm{mho} / \mathrm{m}$ Velocity $\sim 10000 \mathrm{~m} / \mathrm{s}$, Efficiency $\sim 80 \%$ (of kinetic) Power ( comparable size to MarkV) $2000 \mathrm{MWe}$

Fig. 3: Direct fusion yield into working fluids in CFAR MHD [3] -->10 X more energy per $\mathrm{kg}$ than chemical combustion $\rightarrow>10 \times$ higher temperatures $\rightarrow>100 \mathrm{X}$ more power density $\sim \sigma \mathrm{u}^{2}$ than "old" MHD, and $30 \mathrm{X}$ more kWe per ton power density than conventional steam balance-of-plant --> $10 \mathrm{X}$ lower costs! 


\section{ORIGIN := 1 Model for heavy-ion direct-drive T-Lean targets}

Fuel assembly energy (variable), for $\quad i:=1 . .6 \quad$ fuel energy cases, $\quad \operatorname{Ef}_{\mathbf{i}}:=0.2+\sum_{n=1}^{i}[0.2 \cdot(n-1)-0.1]$

DT radius with minimum T loading $\quad \mathbf{f}:=\mathbf{0 . 1} \quad \mathbf{r}_{\text {bound }}\left(\mathbf{r}_{\text {hot }}\right):=\mathbf{r}_{\text {hot }} \cdot(1+2 \cdot \mathbf{f})$

Nominal implosion adiabat $\quad \alpha:=1.5 \quad \zeta:=1-(1+2 \cdot \mathrm{f})^{-3} \quad$ DT load parameter

Use Max's formulary ${ }^{1}$ for fuel assemblies that optimize gain for given fuel energies:

Cold DD fuel density in $\mathrm{g} / \mathrm{cm} 3$ )

$$
\rho_{\text {cdd }}\left(\alpha, \mathbf{E}_{\mathbf{f}}\right):=\mathbf{0 . 8} \cdot 1050 \cdot\left(\alpha^{2} \cdot \mathbf{E}_{\mathbf{f}}\right)^{-0.3}
$$

Hot spot fuel density $(\mathrm{g} / \mathrm{cm} 3)$

$\rho_{\text {hdt }}\left(\mathbf{E}_{\mathbf{f}}\right):=63 \cdot \mathbf{E}_{\mathbf{f}}^{-0.5}$

Eq 2

Hot spot radius $(\mathrm{cm})$

$\mathbf{r}_{\text {hdt }}\left(\mathbf{E}_{\mathbf{f}}\right):=0.0063 \cdot \mathbf{E}_{\mathbf{f}}^{0.5}$

Eq 3

Radius of pure DT region (Case C)

$\mathbf{r}_{\text {bdt }}\left(\mathbf{E}_{\mathbf{f}}\right):=(\mathbf{1}+\mathbf{2} \cdot \mathbf{f}) \cdot \mathbf{r}_{\mathbf{h d t}}\left(\mathbf{E}_{\mathbf{f}}\right)$

Eq 4

Outer DD fuel radius (Tabak) (cm)

$\left.\left.r_{\text {cdd }}\left(E_{f}\right):=\llbracket\left(3 \cdot r_{h d t}\left(E_{f}\right)\right)^{3}-r_{b d t}\left(E_{f}\right)^{3}\right] \cdot 1.25+r_{b d t}\left(E_{f}\right)^{3}\right]^{0.333}$

Eq 5

Mass of cold D fuel (g)

$M_{d}\left(\alpha, E_{f}\right):=4 \cdot 3^{-1} \cdot \pi \cdot\left(r_{c d d}\left(E_{f}\right)^{3}-r_{h d t}\left(E_{f}\right)^{3}\right) \cdot \rho_{c d d}\left(\alpha, E_{f}\right)$

Eq 6

Mass of DT hot spot (g)

$M_{h d t}\left(E_{f}\right):=4 \cdot 3^{-1} \cdot \pi \cdot r_{h d t}\left(E_{f}\right)^{3} \cdot \rho_{h d t}\left(E_{f}\right)$

Eq 7

Total initial T fuel mass (g) (Case C)

$M_{t}\left(\alpha, E_{f}\right):=0.6 \cdot\left(M_{h d t}\left(E_{f}\right)+\frac{\zeta}{0.8} \cdot \frac{4}{3} \cdot \pi \cdot r_{b d t}\left(E_{f}\right)^{3} \cdot \rho_{\text {cdd }}\left(\alpha, E_{f}\right)\right)$

Eq 8

Rho-r of total fuel assembly $\left(\mathbf{g} / \mathrm{cm}^{2}\right) \quad \operatorname{\rho r}\left(\alpha, \mathbf{E}_{\mathbf{f}}\right):=\rho_{\mathbf{h d t}}\left(\mathbf{E}_{\mathbf{f}}\right) \cdot \mathbf{r}_{\mathbf{h d t}}\left(\mathbf{E}_{\mathbf{f}}\right) \ldots$

$$
\begin{aligned}
& +1.25 \cdot \rho_{\mathbf{c d d}}\left(\alpha, \mathbf{E}_{\mathbf{f}}\right) \cdot\left(\mathbf{r}_{\mathbf{b d t}}\left(\mathbf{E}_{\mathbf{f}}\right)-\mathbf{r}_{\mathbf{h d t}}\left(\mathbf{E}_{\mathbf{f}}\right)\right) \ldots \\
& +\rho_{\mathbf{c d d}}\left(\alpha, \mathbf{E}_{\mathbf{f}}\right) \cdot\left(\mathbf{r}_{\mathbf{c d d}}\left(\mathbf{E}_{\mathbf{f}}\right)-\mathbf{r}_{\mathbf{b d t}}\left(\mathbf{E}_{\mathbf{f}}\right)\right)
\end{aligned}
$$

Burnup fractions (Fig.2) $\rho r /\left(\rho r+H_{B}\right)$

depend on $T$ which increases with $\rho r$ :

\begin{tabular}{ccccc}
\hline$f$ & $\begin{array}{c}\text { Main fuel } \\
\text { composition }\end{array}$ & $\begin{array}{c}\text { Column density } \\
\left(\mathrm{g} / \mathrm{cm}^{2}\right)\end{array}$ & $\begin{array}{c}\text { Peak hotspot } \\
\text { temperature } \\
(\mathrm{keV})\end{array}$ & $\begin{array}{c}\text { Main fuel } \\
\text { temperature } \\
(\mathrm{keV})\end{array}$ \\
\hline 0.033 & $\mathrm{D}$ & 4.6 & 40 & 4 \\
0.033 & $\mathrm{D}$ & 12 & 100 & 8 \\
0.166 & $\mathrm{D}$ & 4.6 & 90 & 16 \\
0.166 & $\mathrm{D}$ & 12 & 210 & 50 \\
0.033 & $0.01 \mathrm{~T} / 0.5 \mathrm{D}$ & 4.6 & 70 & 13 \\
0.033 & $0.01 \mathrm{~T} / 0.5 \mathrm{D}$ & 12 & 210 & 80 \\
0.033 & $0.04 \mathrm{~T} / 0.5 \mathrm{D}$ & 4.6 & 180 & 160 \\
0.033 & $0.04 \mathrm{~T} / 0.5 \mathrm{D}$ & 12 & 310 & 160 \\
\hline
\end{tabular}

(Table 2 From Tabak [1]

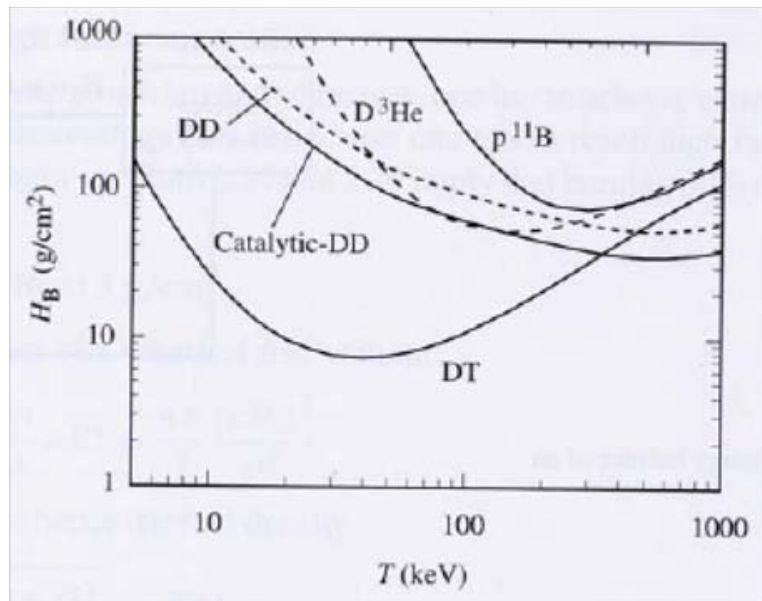

Figure 4: Burnup fractions vs T (from ref. [4]) 
Model for burnup fractions. Note in Table 1 as $\rho r$ increases from 4.6 to $12 \mathrm{~g} / \mathrm{cm} 2$ (factor of 2.6), temperatures for DT hotspot and DD main fuel increase by a similar factors. Fig. 2 shows those temperature changes raise $H_{B}$ for DT $\sim 2$ times, while decreasing $H_{B}$ for DD by $\sim 3$ times. We use this to fit the coefficients for the model $H_{B}$ terms in Eq. 10 and 11 below.

Est burnup fraction of DD (constant

$$
f_{b d}\left(\alpha, E_{f}\right):=\rho r\left(\alpha, E_{f}\right) \cdot\left(\rho r\left(\alpha, E_{f}\right)+1600 \cdot \rho r\left(\alpha, E_{f}\right)^{-1.2}\right)^{-1}
$$
fitted to Tabak's Case C runs).

Est burnup fraction of initial DT load

$$
\mathbf{f}_{\mathbf{b t}}\left(\alpha, \mathbf{E}_{\mathbf{f}}\right):=\rho \mathbf{r}\left(\alpha, \mathbf{E}_{\mathbf{f}}\right) \cdot\left(\rho \mathbf{r}\left(\alpha, \mathbf{E}_{\mathbf{f}}\right)+2 \cdot \rho \mathbf{r}\left(\alpha, \mathbf{E}_{\mathbf{f}}\right)^{\mathbf{0 . 8}}\right)^{-1}
$$

$D(d, p) T$ and $D(d, n) 3 H e$ branching @ 100 keV relative energy

$$
\mathbf{f}_{\mathbf{p}}:=\mathbf{0 . 4 8} \quad \mathbf{f}_{\mathbf{n}}:=\mathbf{0 . 5 2}
$$

Est. yield (MJ) = initial T burnup @ $\mathbf{f}_{\mathrm{bt}} \quad \mathrm{Y}_{\mathbf{f}}\left(\alpha, \mathbf{E}_{\mathbf{f}}\right):=5 \cdot 3^{-1} \cdot \mathbf{M}_{\mathbf{t}}\left(\alpha, \mathbf{E}_{\mathbf{f}}\right) \cdot \mathbf{f}_{\mathrm{bt}}\left(\alpha, \mathrm{E}_{\mathbf{f}}\right) \cdot\left(3.37 \cdot 10^{5}\right) \ldots$

+ D-D burnup yield @ $f_{b d}$

+ bred T burnup yield @ $f_{b t}$

+bred 3He burnup yield assuming

$$
\mathrm{fb}_{3 \mathrm{He}} \sim \mathrm{f}_{\mathrm{bd}} \text {. }
$$

\section{Internal (fuel) energy gains}

$$
\mathbf{G}_{\mathbf{f}}\left(\alpha, \mathbf{E}_{\mathbf{f}}\right):=\mathbf{Y}_{\mathbf{f}}\left(\alpha, \mathbf{E}_{\mathbf{f}}\right) \cdot \mathbf{E}_{\mathbf{f}}^{-1}
$$

M.Tabak results ${ }^{1}$ for fuel gain $G_{m t}$ for his Case $C$ :

$$
\mathbf{n}:=1 . .7
$$

$$
\text { Gmt } 1:=105 \quad \text { Gmt }_{2}:=160 \quad \text { Gmt }_{3}:=230 \quad \text { Gmt }_{4}:=295 \quad \text { Gmt5 }:=360 \quad \text { Gmt }_{6}:=430 \quad \text { Gmt } 7:=500
$$

$\mathrm{E}_{1}:=0.1 \quad \mathrm{E}_{2}:=0.25 \quad \mathrm{E}_{3}:=0.5 \quad \mathrm{E}_{4}:=0.85 \quad \mathrm{E}_{5}:=1.3 \quad \mathrm{E}_{6}:=1.85 \quad \mathrm{E}_{7}:=2.5$

Fig. 5: Comparing fuel gain $G_{f}$ (model) and in Tabak's Case $C$ vs. fuel energy $E_{f}$ (At adiabat $\alpha=2$ )

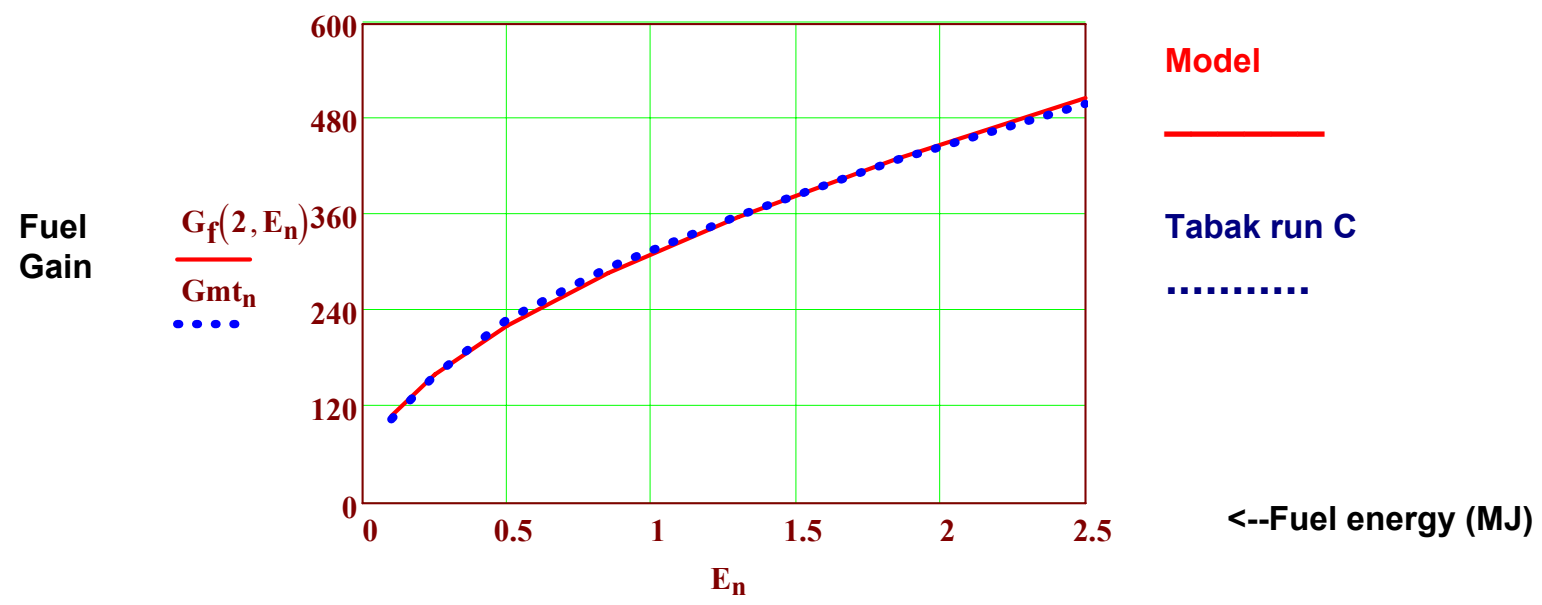

Fig. 5 shows the model and the Case $C$ runs agree well. (Good enough for government work). 


\section{Next lets consider T-breeding:}

Mass of T-bred in-situ by D+D-->T+p branch (g) $\quad M_{t b}\left(\alpha, E_{f}\right):=0.75 \cdot f_{p} \cdot M_{d}\left(\alpha, E_{f}\right) \cdot f_{b d}\left(\alpha, E_{f}\right)$

Eq 14

Mass of $3 \mathrm{He}-$ bred by $\mathrm{D}+\mathrm{D}-->3 \mathrm{He}+\mathrm{n}$ branch $(\mathrm{g})$

$\mathbf{M}_{3 \mathrm{Heb}}\left(\alpha, \mathbf{E}_{\mathbf{f}}\right):=\mathbf{0 . 7 5} \cdot \mathbf{f}_{\mathbf{n}} \cdot \mathbf{M}_{\mathbf{d}}\left(\alpha, \mathbf{E}_{\mathbf{f}}\right) \cdot \mathbf{f}_{\mathbf{b d}}\left(\alpha, \mathbf{E}_{\mathbf{f}}\right)$

Eq 15

Total T consumed by fusion $(g)$

$\mathbf{M}_{\mathbf{t c}}\left(\alpha, \mathbf{E}_{\mathbf{f}}\right):=\left(\mathbf{M}_{\mathbf{t}}\left(\alpha, \mathbf{E}_{\mathbf{f}}\right)+\mathbf{M}_{\mathbf{t b}}\left(\alpha, \mathbf{E}_{\mathbf{f}}\right)\right) \cdot \mathbf{f}_{\mathbf{b t}}\left(\alpha, \mathbf{E}_{\mathbf{f}}\right)$

Eq 16

Net T mass gain (loss) (g) $\quad \mathbf{M}_{\mathrm{tn}}\left(\alpha, \mathbf{E}_{\mathbf{f}}\right):=\mathbf{M}_{\mathrm{tb}}\left(\alpha, \mathbf{E}_{\mathbf{f}}\right)-\mathbf{M}_{\mathrm{tc}}\left(\alpha, \mathbf{E}_{\mathbf{f}}\right)$

Eq 17

In-situ Neutrons per Triton burned

$\mathbf{N}_{\text {ndd }}\left(\alpha, \mathbf{E}_{\mathbf{f}}\right):=\left(\mathbf{M}_{3 \mathbf{H e b}}\left(\alpha, \mathbf{E}_{\mathbf{f}}\right)+\mathbf{M}_{\mathbf{t c}}\left(\alpha, \mathbf{E}_{\mathbf{f}}\right)\right) \cdot \mathbf{M}_{\mathbf{t c}}\left(\alpha, \mathbf{E}_{\mathbf{f}}\right)^{-1}$

Eq 18

Note if all tritium were burned up, and the DD breeding of T dominated over the initial T load, the maximum $\mathbf{N}_{\mathrm{nt}} \max =2$. However, about 50 percent of tritium is burned, so the maximum $\mathbf{N}_{\mathrm{nt}}<3$, including some initial $\mathrm{T}$ load with the parameter $\mathrm{f}=0.1$.

No. of neutrons available (inc. multiplication and minus any needed for T-replacement) per T burned:

$$
\begin{array}{cl}
\text { For T-lean case } & \mathbf{N}_{\text {ndda }}\left(\alpha, \mathbf{E}_{\mathbf{f}}\right):=\left(\mathbf{M}_{3 \mathrm{Heb}}\left(\alpha, \mathbf{E}_{\mathbf{f}}\right)+\mathbf{1 . 4} \cdot \mathbf{M}_{\mathrm{tc}}\left(\alpha, \mathbf{E}_{\mathbf{f}}\right)\right) \cdot \mathbf{M}_{\mathrm{tc}}\left(\alpha, \mathbf{E}_{\mathbf{f}}\right)^{-1} \\
\text { For DT case } & \mathbf{N}_{\mathbf{n d t a}}:=\mathbf{1 . 4 - 1}
\end{array}
$$

No. of neutrons available for uses other than $\mathrm{T}$ replacement, per $\mathrm{MeV}$ of fusion yield

$$
\begin{aligned}
& \text { For T-lean case } \quad \mathrm{NY}_{\mathbf{d d}}\left(\alpha, \mathrm{E}_{\mathbf{f}}\right):=\mathrm{N}_{\text {ndda }}\left(\alpha, \mathrm{E}_{\mathbf{f}}\right) \cdot\left(\frac{\mathrm{M}_{\mathbf{d}}\left(2, \mathrm{E}_{\mathbf{f}}\right) \cdot 4.6+\mathrm{M}_{\mathbf{t}}\left(2, \mathrm{E}_{\mathbf{f}}\right) \cdot 17.6}{M_{d}\left(2, \mathrm{E}_{\mathbf{f}}\right)+\mathrm{M}_{\mathbf{t}}\left(2, \mathrm{E}_{\mathbf{f}}\right)}\right)^{-1} \\
& \text { For DT case } \quad \mathrm{NY}_{\mathrm{dt}}\left(\alpha, \mathrm{E}_{\mathrm{f}}\right):=\mathrm{N}_{\text {ndta }} \cdot \mathbf{1 7 . 6}^{-1}
\end{aligned}
$$

Ratio of neutrons available T-lean case over DT case, per MeV yield

$$
\operatorname{NpYR}\left(\alpha, \mathbf{E}_{\mathbf{f}}\right):=\mathrm{NY}_{\mathbf{d d}}\left(\alpha, \mathbf{E}_{\mathbf{f}}\right) \cdot \mathbf{N Y} \mathbf{d t}_{\mathbf{f t}}\left(\alpha, \mathbf{E}_{\mathbf{f}}\right)^{-1}
$$

Table 3: Model predictions for net tritium production versus fuel energy. (Adiabat $\alpha=1.5$ )

Fuel energy

$$
\begin{aligned}
& \text { Initial T } \\
& \text { mass }
\end{aligned}
$$

$$
\begin{aligned}
& \mathbf{M}_{\mathbf{t}}(\mathbf{1 . 5}, \\
& \hline \mathbf{1 0}^{-\mathbf{3}} \\
& \hline 0.03 \\
& \hline 0.06 \\
& \hline 0.18 \\
& \hline 0.42 \\
& \hline 0.78 \\
& \hline 1.29 \\
& \hline
\end{aligned}
$$

(mg)

(MJ)
T-mass bred
T-mass consumed
Net T--Mass produced
Neutrons per T burned

$\mathbf{i}=$
\begin{tabular}{|r|}
\hline \\
\hline 2 \\
\hline 3 \\
\hline 4 \\
\hline 5 \\
\hline 6 \\
\hline
\end{tabular}

$$
\begin{gathered}
=\frac{\mathbf{M}_{\mathbf{t b}}(\mathbf{1 . 5}}{\mathbf{1 0}^{-3}} \\
\begin{array}{|c|}
\hline 0.02 \\
\hline 0.06 \\
\hline 0.24 \\
\hline 0.71 \\
\hline 1.64 \\
\hline 3.21 \\
\hline
\end{array}
\end{gathered}
$$

(mg)

$$
=\frac{M_{t c}\left(1.5, E_{i}\right)}{10^{-3}}=\frac{M_{t n}\left(1.5, E_{i}\right)}{10^{-3}}=
$$

\begin{tabular}{|r|}
\hline 0.02 \\
\hline 0.05 \\
\hline 0.18 \\
\hline 0.5 \\
\hline 1.08 \\
\hline 2.03 \\
\hline
\end{tabular}

(mg)

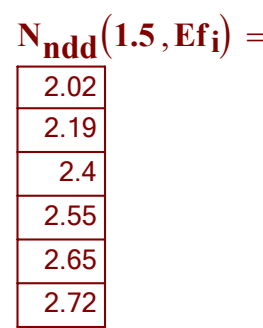

Note in Table 3 that net tritium self-breeding sufficiency occurs for fuel energies $\sim>0.2 \mathrm{MJ}$ for these cases with $1.3 \%$ molar fraction of tritium. Thus $E_{f}=0.2 \mathrm{MJ}$ will be our reference DEMO case. 
Table 4 compares the Tabak-based model @ adiabat $\alpha=1.5$ with the closest Atzeni/Ciampi's T-lean example [2] for marginal net T-sufficiency at adiabat $\alpha=1.5$, both cases near $1 \%$ molar fraction of tritium, and for $1 \mathrm{MJ}$ fuel assembly energy (which will be our reference case for a CFAR power plant. The Tabak-based model is based on isobaric DT hotspot ignition, while the Atzeni/Ciampi model is based on isochoric fast ignition. We assume the Tabak model would also be consistent with the new Betti-Perkins variant of hot spot ignition with a late shock but without needing a fast igniter pulse (easier for ion beam drive), in case implosions don't quite reach the $10 \mathrm{keV}$ hot spot DT temperatures postulated in the beginning of Max's burn calculations.

Table 4

Molar T-fraction

Adiabat $\alpha$

Fuel energy (MJ)

Fuel Mass (g)

Fuel density $\left(\mathrm{g} / \mathrm{cm}^{3}\right)$

Fuel Rho-r (g/cm $\left.{ }^{2}\right)$

Fuel gain $\mathrm{G}_{\mathrm{f}}$ and

Fusion Yield (in MJ)
Atzeni/Ciampi example

$1 \%$

1.5

1

0.020

800

10.7

1050

\section{Tabak-based MCAD Model}

$1.3 \%$

1.5

1

$M_{d}(1.5,1)+M_{t}(1.5,1)=0.023$

$\rho_{\text {cdd }}(1.5,1)=659$

$\operatorname{\rho r}(1.5,1)=9.9$

$\mathbf{G}_{\mathbf{f}}(1.5,1)=494$

Note that despite the difference in the two ignition/burn models, these cases compare reasonably at similar dd fuel masses and fuel energies at comparable seed molar tritium fractions and both at marginal T self-breeding sufficiency. If anything, the Tabak-based model is pessimistic with respect to the Atzeni/Ciampi calculation. We should expect the higher fuel gain for the Atzeni/Ciampi isochoric ignition assumption compared to the isobaric ignition Tabak-based MCAD model. We prefer the latter approach anyway because its easier to drive with $\mathrm{mm}$-spot radius ion beams. Fig. 6 compares neutrons per $\mathrm{T}$ burned and extra available neutrons (neutrons produced minus any needed for $\mathrm{T}$ breeding) per $\mathrm{MeV}$ of fusion yield for the T-lean assembles compared to conventional DT targets, where we assume a typical DT neutron multiplication of 1.4 for FLiBe blankets, but no multiplication for any dd neutrons.

Fig 6: Neutrons produced per $\mathrm{T}$ burned and the ratio of available neutrons per MeV yield vs fuel energy.

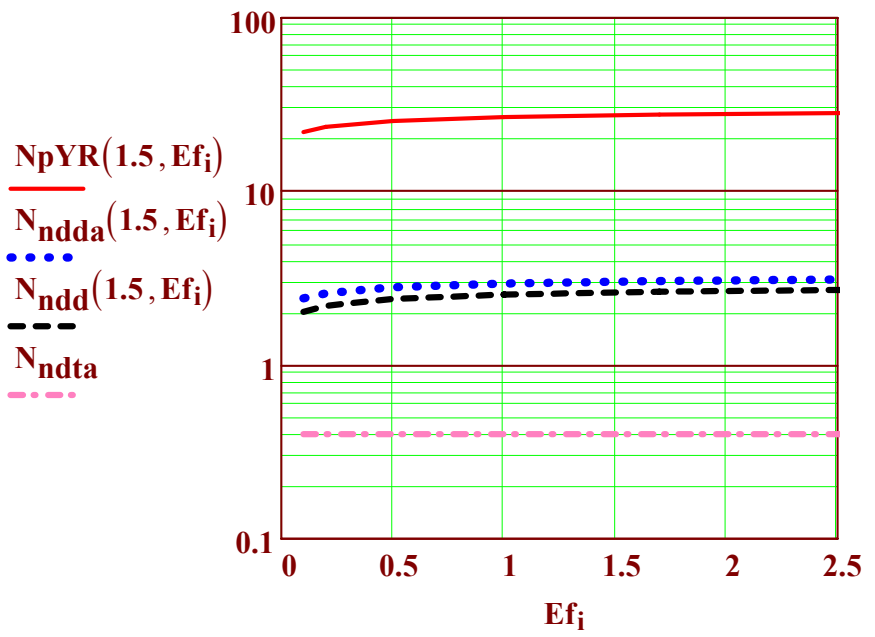

(Adiabat $\alpha=1.5$ )

Ratio of available neutrons per MeV yield (ratio of T-lean case over the pure DT case)

\# neutrons produced per T burned \# in-situ neutrons per T burned (T-lean cases)

\# neutrons available per T burned (DT case only) <--Fuel energy (MJ)

Figure 6 shows that T-lean targets can be 30 times more prolific neutron sources per fusion watt for purposes of various applications in blankets compared to DT targets, besides being tritium self-sufficient (sufficient in-situ breeding so that blanket material options are not restricted to contain lithium). 
The copious extra neutrons available with T-lean targets, as pointed out by Tabak ${ }^{1}$, can be used in external blankets of different materials, (in some cases without lithium) for several purposes:

(a) generate extra energy for direct conversion through exothermic neutron capture

(b) generate extra tritium for sale to other tritium-deficient reactors like ITER

(c) breed fissile fuel sufficient to support many client fission reactors

Direct MHD conversion. Next, lets look at the potential to use these T-lean cases for low-cost Balance-of-Plant with direct plasma MHD conversion, based on the Compact Fusion Advanced Rankine (CFAR) cycle ${ }^{2}$, in which a small vaporizable/ionizable/recylable shell material is inserted around and simultaneously with each target (See Fig. 8 below).

Scaling of capture fractions of T-lean target outputs into shells for plasmas Fraction of yield born in neutrons (neglecting inelastic neutron scattering) (un-attenuated neutrons)

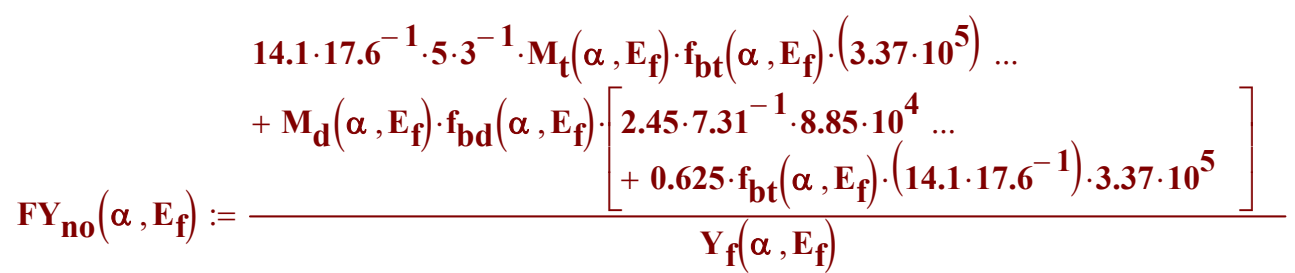

Figure 7 Neutron energy loss in hydrogen component of target shells

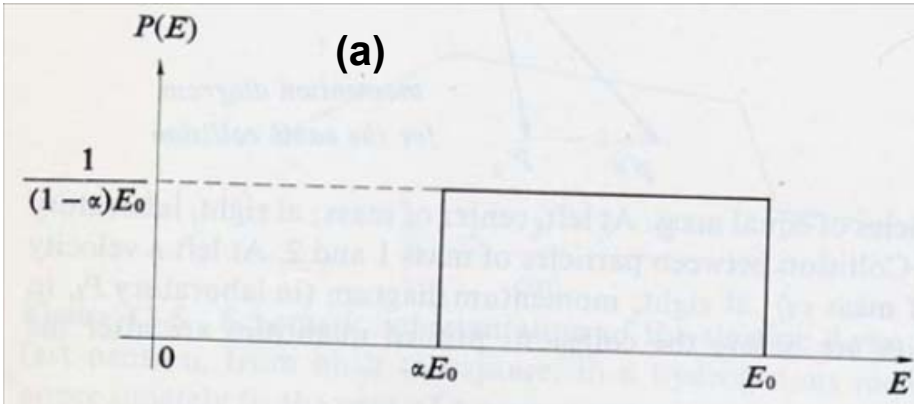

Distribution of the values of the neutron energy $E$ after one collision in the case of isotropic scattering in the centerof-mass system for a neutron of initial energy $E_{0}$. The quantity $\alpha=(A-1)^{2} /(A+1)^{2}$.

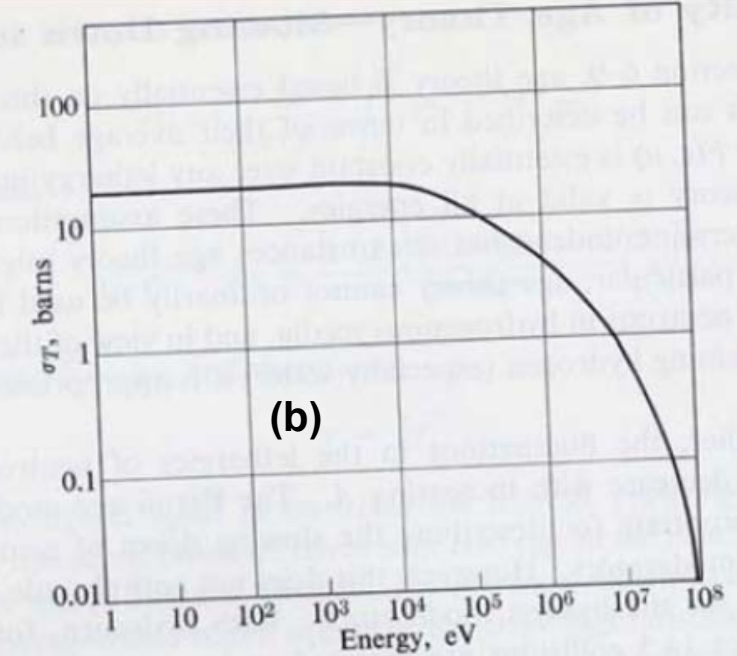

The total cross section of hydrogen.
Note for hydrogen in the target shell, this $\alpha=0$, for deuterium in the target, $\alpha=0.11$. For hydrogen/deuterium, neutrons don't diffuse much further from the point they have their first inelastic collision. For $14 \mathrm{MeV}$ neutrons from DT, the efolding $\rho r$ in hydrogen is $5 \mathrm{~g} / \mathrm{cm}^{2}$, or $10 \mathrm{~g} / \mathrm{cm}^{2}$ in deuterium, neglecting the neutron's cross section contribution in the deuterium nucleus. For $2.5 \mathrm{MeV}$ neutrons from $D D$, the e-folding $\rho r$ is about $0.7 \mathrm{~g} / \mathrm{cm}^{2}$ in $\mathrm{H}$, or $1.4 \mathrm{~g} / \mathrm{cm}^{2}$ in D. As we will see, the T-lean targets reduce the escaping neutron energy $\sim 50 \%$ as in this Fig $5(\mathrm{a})$, going into the shell. --->Estimated neutron e-folding $\rho r$ 's:

$$
\begin{array}{lll}
\rho r_{14 D}:=10 & \rho r_{14 H}:=5 & \rho r_{7 H}:=2 \\
\rho r_{2.5 D}:=1.4 & \rho r_{2.5 H}:=0.7 & \rho r_{1.2 H}:=0.4 \\
\text { in the target } & \text { all } \mathbf{g} / \mathbf{c m}^{2} & \text { in the shell }
\end{array}
$$



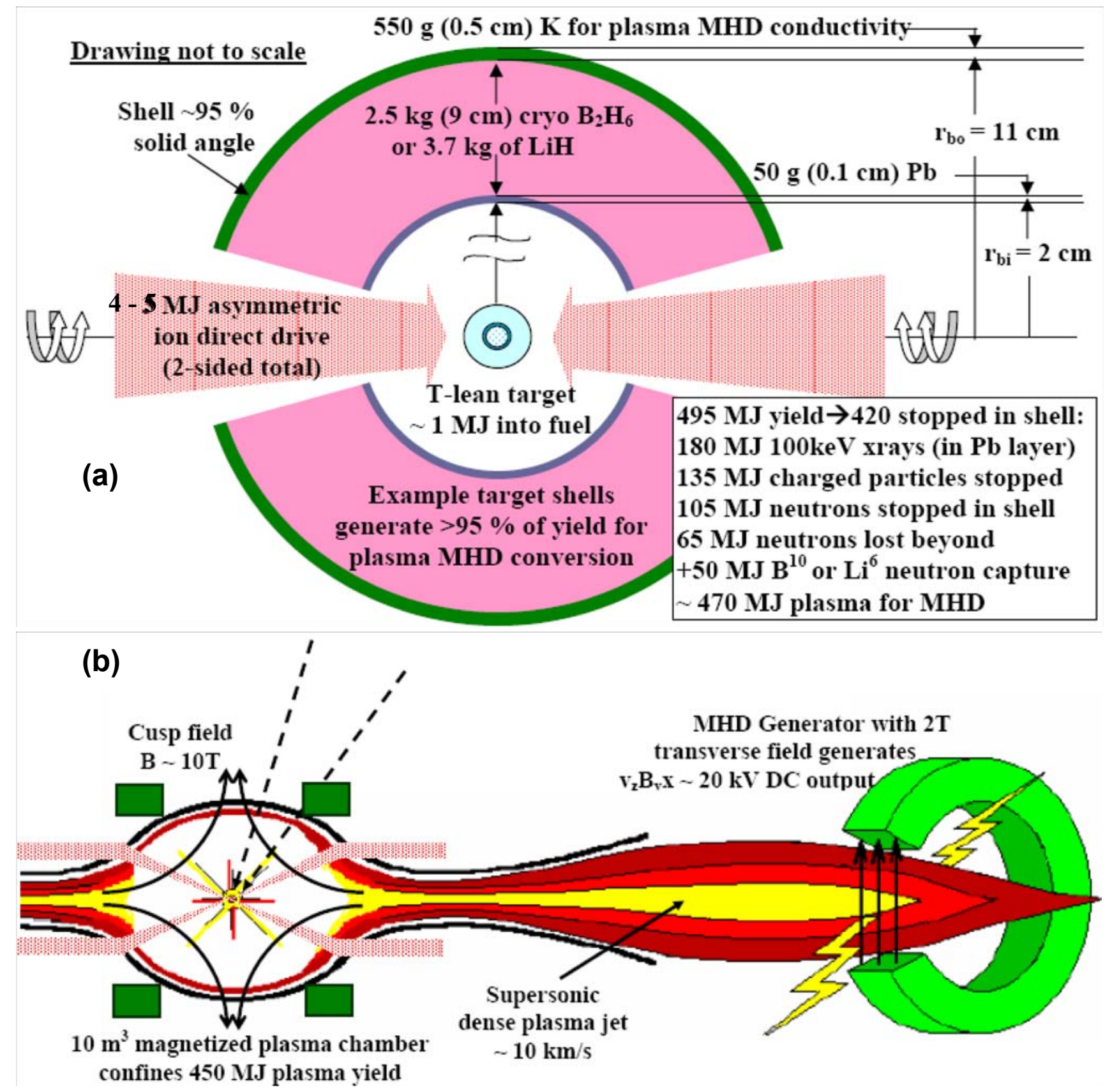

Figure 8: (a) Example target shell for efficient conversion of T-lean target output into 1 to $2 \mathrm{eV}$ dense plasma for direct MHD conversion. All shell materials condense and recycle (Rankine cycle).

(b) Schematic of the CFAR MHD scheme (adapting the old 1992 CFAR Logo!)--no detailed design yet.

The shell has $5 \%$ solid angle holes for driver beam access, and is used to capture target chargedparticle, $\mathrm{x}$-ray, and at least half of the remaining neutron energy escaping the target to create 1 to $2 \mathrm{eV}$ dense chamber plasma for direct conversion. (n contrast to ref. 3, most of the neutron energy is internally captured in T-lean cases because the fuel $\rho$ r exceeds the neutron mean-free-path. Also, a thin $(1 \mathrm{~mm})$ lead layer lines the inner surface of the target shell to capture the $40 \%$ of T-lean target output in the form of $100 \mathrm{keV}$ x-rays. The bulk of the target shell consists of some hydrogen hydride to efficiently absorb neutron energy (which may also provide some energy multiplication through exothermic neutron capture), and is capped by an outer layer of alkali metal such as Potassium to enhance the plasma conductivity of the subsequent mix for efficient ( $>50 \%$ direct) MHD conversion. 
Additional facts about Fig. 8- the marriage of T-lean targets to CFAR energy conversion: A 10 T cusp field on a $2-\mathrm{m}$ vessel/coil radius with a protective $0.6 \mathrm{~m}$-thick Flibe vortex layer inside aids final focusing of ion beams and prevents large shocks to the vessel wall with large yields. The plasma created from the T-lean target shell is conductive enough for the field to confine the $\mathbf{4 7 0} \mathrm{MJ}$ plasma until drained out through the MHD generators in $\sim 50 \mathrm{~ms}$. Unlike ref. 3, we assume here $<30 \%$ duty factor for MHD generation to allow the chamber pressure to drop to low values for target insertion. $65 \mathrm{MJ}$ of neutrons $+15 \mathrm{MJ}$ of $\mathrm{x}$-rays not stopped in the target shell act like a small $80 \mathrm{MJ}$ yield in the $10 \mathrm{~m}^{3}$ Flibe vortex pocket.

\section{Neutron energy attenuation factors A.}

The average $\rho r$ a neutron has to go to escape in the target depends on where it is born, so we need to define some spatial weighting factors fs when using the total target fuel $\rho r$ :

$\begin{array}{llll}\text { fsdti }:=1 & A_{n t}\left(\alpha, E_{\mathbf{f}}\right):=\exp \left(-\mathbf{f s d t i} \cdot \rho r\left(\alpha, E_{\mathbf{f}}\right) \cdot \rho r_{14 D}{ }^{-1}\right) & \text { for DT neutrons from hot spot } & \text { Eq } 25 \\ \text { fsddo }:=\mathbf{0 . 2} & A_{n d}\left(\alpha, E_{\mathbf{f}}\right):=\exp \left(-\mathbf{f s d d o} \cdot \rho r\left(\alpha, E_{\mathbf{f}}\right) \cdot \rho r_{2.5 D}{ }^{-1}\right) & \text { outer-born DD neutrons } & \text { Eq 26 } \\ \text { fsdto }:=\mathbf{0 . 4} & A_{\text {ndt }}\left(\alpha, E_{\mathbf{f}}\right):=\exp \left(-\mathbf{f s d t o} \cdot \rho r\left(\alpha, E_{\mathbf{f}}\right) \cdot \rho r_{14 D}{ }^{-1}\right) & \text { outer-born DT neutrons } & \text { Eq 27 }\end{array}$

The weighting factor for $2.5 \mathrm{MeV}$ deuterons should be less than the dd-mass weighting 0.33 , while the outer-born DT neutrons should be a bit more than 0.33 , because the burn wave $T$ increases with $r$. Model fraction of fusion energy escaping as neutrons [(1- FY) would be the fraction captured]

$$
\begin{aligned}
& 14.1 \cdot 17.6^{-1} \cdot 5 \cdot 3^{-1} \cdot M_{t}\left(\alpha, E_{f}\right) \cdot f_{b t}\left(\alpha, E_{f}\right) \cdot\left(3.37 \cdot 10^{5}\right) \cdot A_{n t}\left(\alpha, E_{f}\right) \ldots \\
& +M_{d}\left(\alpha, E_{f}\right) \cdot f_{b d}\left(\alpha, E_{f}\right) \cdot\left[\begin{array}{l}
2.45 \cdot 7.31^{-1} \cdot 8.85 \cdot 10^{4} \cdot A_{n d}\left(\alpha, E_{f}\right) \ldots \\
+0.625 \cdot f_{b t}\left(\alpha, E_{f}\right) \cdot\left(14.1 \cdot 17.6^{-1}\right) \cdot 3.37 \cdot 10^{5} \cdot A_{n d t}\left(\alpha, E_{f}\right)
\end{array}\right]
\end{aligned}
$$

M.Tabak results for escaping neutron fraction of yield for his Case C: $\quad$ FYmt $1:=0.45 \quad$ FYmt $2:=0.4$

$$
\text { FYmt } 3:=0.35 \quad \text { FYmt }_{4}:=0.33 \quad \text { FYmt } 5:=0.31 \quad \text { FYmt }_{6}:=0.30 \quad \text { FYmt }_{7}:=0.29
$$

Now lets add the additional neutron capture in the target shell. To maintain the optimum shell-produced plasma temperature of $1.5 \mathrm{eV}$ for MHD, we need to scale the shell-blanket mass (see figure 8) proportional to captured fusion yield to keep the average energy deposition in the shell $110 \mathrm{MJ} / \mathrm{kg}$. To simply the estimate, we note that the captured fusion yield is close enough to the fusion yield that we can scale the outer shell-blanket radius $\mathrm{r}_{\mathrm{bo}}$ simply as:

$$
\mathbf{r}_{\mathbf{b o}}\left(\alpha, \mathbf{E}_{\mathbf{f}}\right):=11 \cdot\left(\mathbf{Y}_{\mathbf{f}}\left(\alpha, \mathbf{E}_{\mathbf{f}}\right) \cdot \mathbf{Y}_{\mathbf{f}}(1.5,1)^{-1}\right)^{0.333}
$$

The resulting additional attenuation (capture) of neutron energy for direct conversion purposes (fraction of yield finally escaping both the target and the shell), estimating attenuation by hydrogen component density in the shell, and augmenting that by $25 \%$ to account for Boron/Lithium capture:

$$
\begin{aligned}
& \rho_{\mathbf{H b}}:=0.12 \quad \mathbf{g} / \mathrm{cm}^{3} \quad \text { density of hydrogen in blanket shell } \\
& A_{n t b}\left(\alpha, E_{f}\right):=\exp \left[-1.25 \cdot \rho_{H b} \cdot\left(r_{b o}\left(\alpha, E_{f}\right)-2\right) \cdot \rho r_{7 H}-1\right] \\
& A_{n d b}\left(\alpha, E_{f}\right):=\exp \left[-1.25 \cdot \rho_{\mathbf{H b}} \cdot\left(r_{b o}\left(\alpha, E_{f}\right)-2\right) \cdot \rho r_{1.2 H}-1\right]
\end{aligned}
$$




$$
\begin{aligned}
& 14.1 \cdot 17.6^{-1} \cdot 5 \cdot 3^{-1} \cdot M_{t}\left(\alpha, E_{f}\right) \cdot f_{b t}\left(\alpha, E_{f}\right) \cdot\left(3.37 \cdot 10^{5}\right) \cdot A_{n t}\left(\alpha, E_{f}\right) \cdot A_{n t b}\left(\alpha, E_{f}\right) \ldots \\
& +M_{d}\left(\alpha, E_{f}\right) \cdot f_{b d}\left(\alpha, E_{f}\right) \cdot\left[2.45 \cdot 7.31^{-1} \cdot 8.85 \cdot 10^{4} \cdot A_{n d}\left(\alpha, E_{f}\right) \cdot A_{n d b}\left(\alpha, E_{f}\right) \ldots\right. \\
& \frac{\left[+0.625 \cdot f_{b t}\left(\alpha, E_{f}\right) \cdot\left(\frac{14.1}{17.6}\right) \cdot 3.37 \cdot 10^{5} \cdot A_{n d t}\left(\alpha, E_{f}\right) \cdot A_{n t b}\left(\alpha, E_{f}\right)\right]}{Y_{f}\left(\alpha, E_{f}\right)}
\end{aligned}
$$

\begin{tabular}{|c|c|c|c|c|c|}
\hline $\mathbf{E} \mathbf{f}_{\mathbf{i}}=$ & $\operatorname{\rho r}(1.5$ & $\mathbf{Y}_{\mathbf{f}}\left(\mathbf{1 . 5}, \mathbf{E} \mathbf{f}_{\mathbf{i}}\right)=$ & $\mathbf{r}_{\mathbf{b o}}\left(1.5, \mathbf{E f _ { \mathbf { i } }}\right)=$ & $F Y_{n b}\left(1.5, \mathbf{E f}_{\mathbf{i}}\right)=$ & $1-F Y_{n b}\left(1.5, \mathbf{E f}_{\mathbf{i}}\right)=$ \\
\hline 0.1 & 6.4 & 16 & 3.5 & 0.34 & 0.66 \\
\hline 0.2 & \begin{tabular}{|l|}
7.3 \\
\end{tabular} & 43 & 4.9 & 0.27 & 0.73 \\
\hline 0.5 & 8.6 & 171 & 7.7 & 0.19 & 0.81 \\
\hline 1 & 9.9 & 494 & 11 & 0.14 & 0.86 \\
\hline 1.7 & 10.9 & 1124 & 14.5 & 0.1 & 0.9 \\
\hline 2.6 & 11.8 & 2181 & 18 & 0.07 & 0.93 \\
\hline
\end{tabular}

Table 5: Target shell radius $r_{b o}$ \& fusion yield loss fraction $F Y_{n b}$ vs fuel energy $E_{f}$ (Adiabat $\alpha=1.5$ )
MJ
$\mathrm{g} / \mathrm{cm}^{2}$
MJ
$\mathbf{c m}$
Yield fraction lost Yield fraction captured

Figure 9. Fraction of total T-lean target yield escaping target and shell as neutrons as a function of fuel energy $E_{f}$ (adiabat $\alpha=2$ ). The model (solid red curve), and Max Tabak's case $C$ runs agree well and show significant reduction of lost neutron energy below the un-attenuated neutron yield (blue dotted line) within the large $\rho r$ of T-lean targets. The shell captures $\sim 50 \%$ of remaining neutron energy escaping the target (depending on the fuel $\rho$ r- see dashed magenta curve for $F Y_{n b}$ ).

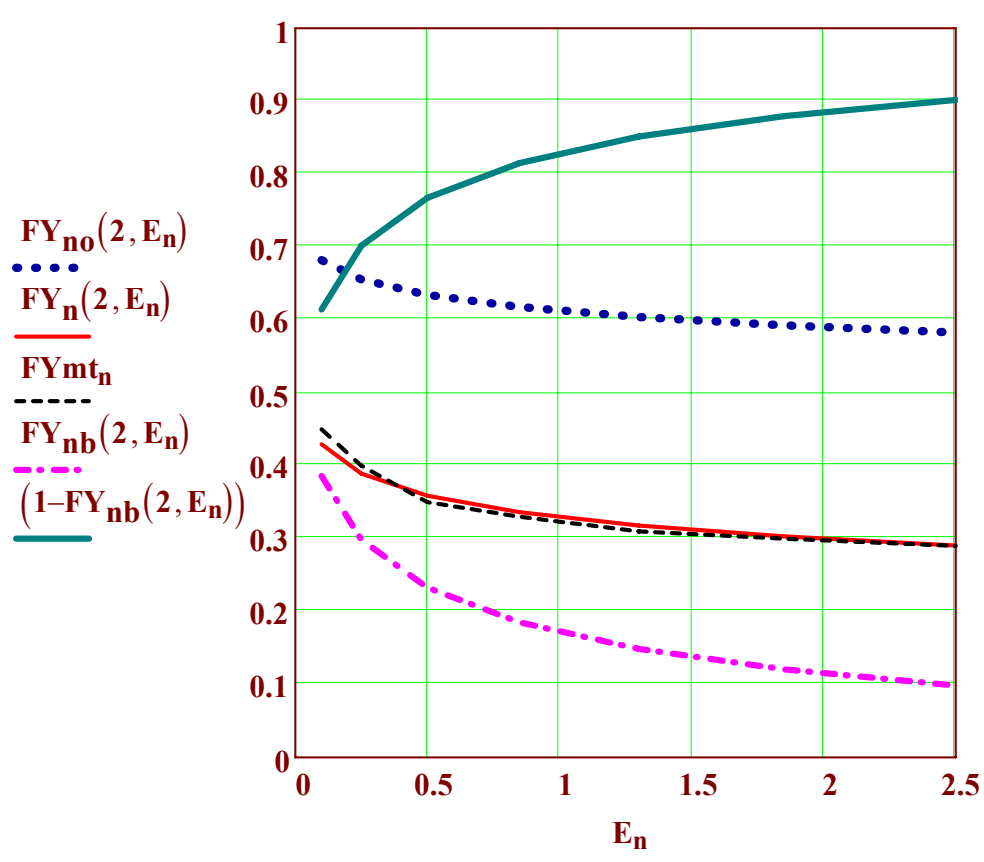

Fraction of yield captured in target + target shell (Model)

\section{Un-attenuated neutron energy} as a fraction of yield (model)

Max Tabak's Case $C$ neutron energy escaping the target as fraction of yield (black dashed) compared to model (red curve).

Fraction of neutron energy escaping both target and target shell (model)

<--Fuel energy (MJ)

Page 13 
Rocket efficiency and implosion efficiency with pure H2 ablators.

We assume a pure hydrogen ablator for ion beam direct drive because hydrogen provides the highest exhaust velocity for ablative drive for a given specific energy deposition by short range ions, as well as having the smallest specific ionization energy loss per specific radial kinetic energy of exhaust $0.5 \mathrm{u}_{\mathrm{ex}}{ }^{2}$. Working backwards, we will estimate ion beam drive requirements shortly. We assume thin coatings between the DT, DD, and $\mathrm{H}$ ablator layers such that the hot spot decay heat gives equilibrium temperatures of 19-->14-->10 deg $\mathrm{K}$ for these layers respectively. The DT layer would be filled through the hydrogen layer by a thin fill tube. (see Figure 12 below)

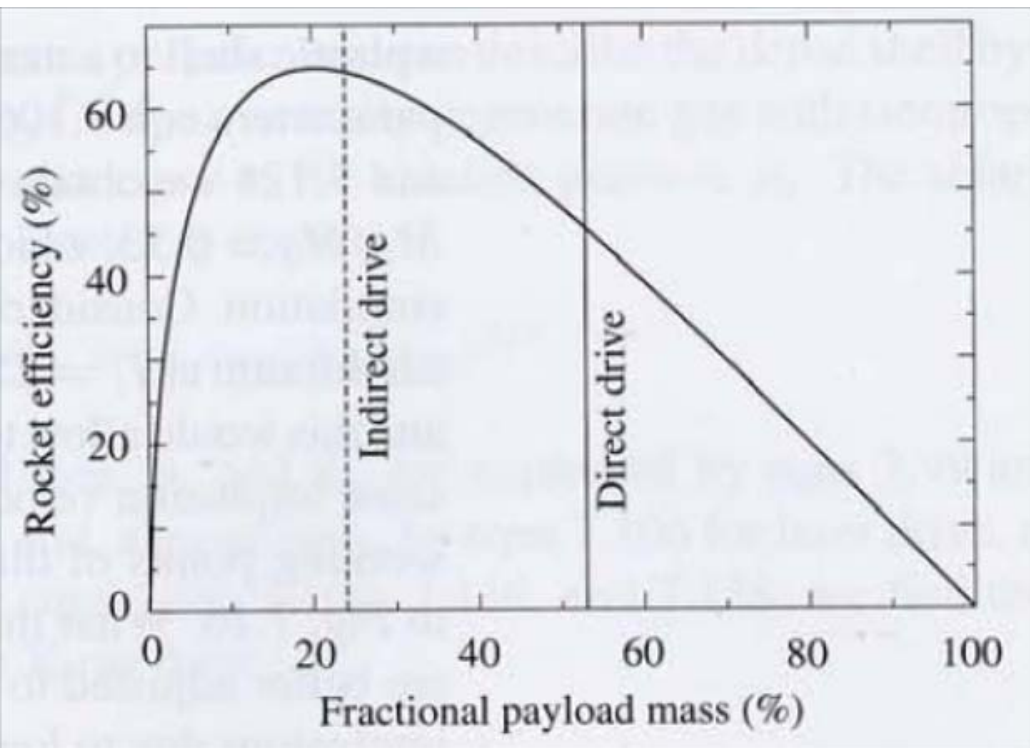

Figure 10: the classic spherical rocket efficiency $\eta_{r}$ as a function of the fractional payload mass (Figure from the book by Meyer-ter-Vehn and Atzeni)

An issue is managing space charge in accelerators delivering the energy with short enough range ions to allow pulse shaping for low adiabats. Another factor can be ionization energy losses and radiation losses which can reduce the capsule hydro efficiency $\eta_{c}$ below the rocket efficiency $\eta_{\mathrm{c}}$. However, pure hydrogen has a small ionization energy of $13.6 \mathrm{eV}$ per atom. So, from Fig. 10, we choose to drive at the peak rocket efficiency $\eta_{r}:=0.65$

with an associated fractional payload mass $M_{1}=0.2 M_{0}$, which requires an $H_{2}$ ablator mass $M_{h}$ :

$$
\mathbf{M}_{\mathbf{h}}\left(\alpha, \mathbf{E}_{\mathbf{f}}\right):=\mathbf{4} \cdot\left(\mathbf{M}_{\mathbf{d}}\left(\alpha, \mathbf{E}_{\mathbf{f}}\right)+\mathbf{M}_{\mathbf{t}}\left(\alpha, \mathbf{E}_{\mathbf{f}}\right)\right)
$$

The implosion velocity $u_{i m p}$ is that required to create the fuel internal energy $E_{f}$ upon stagnation:

$$
\mathbf{u}_{\mathbf{i m p}}\left(\alpha, \mathbf{E}_{\mathbf{f}}\right):=\left[2 \cdot \mathbf{E}_{\mathbf{f}} \cdot 10^{13} \cdot\left(\mathbf{M}_{\mathbf{d}}\left(\alpha, \mathbf{E}_{\mathbf{f}}\right)+\mathbf{M}_{\mathbf{t}}\left(\alpha, \mathbf{E}_{\mathbf{f}}\right)\right)^{-1}\right]^{0.5} \quad(\mathrm{~cm} / \mathbf{s}) \text {. }
$$

The ablation exhaust velocity $u_{e x}$ for the chosen peak rocket efficiency is given by

$$
u_{\text {ex }}\left(\alpha, E_{f}\right):=u_{i m p}\left(\alpha, E_{f}\right) \cdot \ln (5)^{-1} \quad(\mathrm{~cm} / \mathrm{s})
$$




\section{Radiation losses and avoidance of preheat}

We estimate the hydrogen ablator compresses by about a factor of 5 during much of the direct drive period, to an average density around $0.5 \mathrm{~g} / \mathrm{cm}^{2}$. The $\mathrm{H}_{2}$ temperature just behind the ablation front can be estimated by setting $u_{e x}=2 c_{s}$ and solving for $T$ :

$$
\rho_{\mathrm{Ho}_{0}}:=0.1 \quad \mathrm{~g} / \mathrm{cm}^{3} \text { for } \mathrm{H}_{2} \text { ablator } \rho_{0}
$$$$
\rho_{\mathbf{a}}:=\mathbf{5} \cdot \rho_{\mathbf{H o}}
$$

$$
\mathrm{m}_{\mathrm{h}}:=1.67 \cdot 10^{-24} \mathrm{~g} / \mathrm{H} \text { atom }
$$

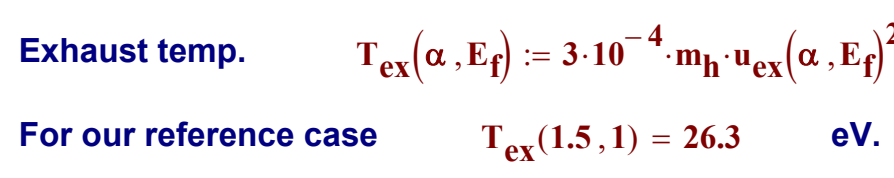

$$
\begin{aligned}
& \begin{array}{llll}
u_{\text {imp }}(1.5,1)=2.95 \times 10^{7} & \mathrm{~cm} / \mathrm{s} & \frac{u_{i m p}(1.5,1)}{u_{e x}(1.5,1)}=1.61 & \operatorname{Mr} / M_{f}=5 \\
u_{e x}(1.5,1)=1.83 \times 10^{7} & \mathrm{~cm} / \mathrm{s} & \ln (5)=1.61
\end{array}
\end{aligned}
$$

Figure 11 shows that under these conditions (large ablation fractions at the peak of rocket efficiency), and initial $\mathrm{H} 2$ ablator $\rho r \sim 0.02 \mathrm{~g} / \mathrm{cm}^{2}$ vs $\kappa_{R}^{-1}=3 \times 10^{-4} \mathrm{~g} / \mathrm{cm}^{2}$, that such ablators are optically thick.

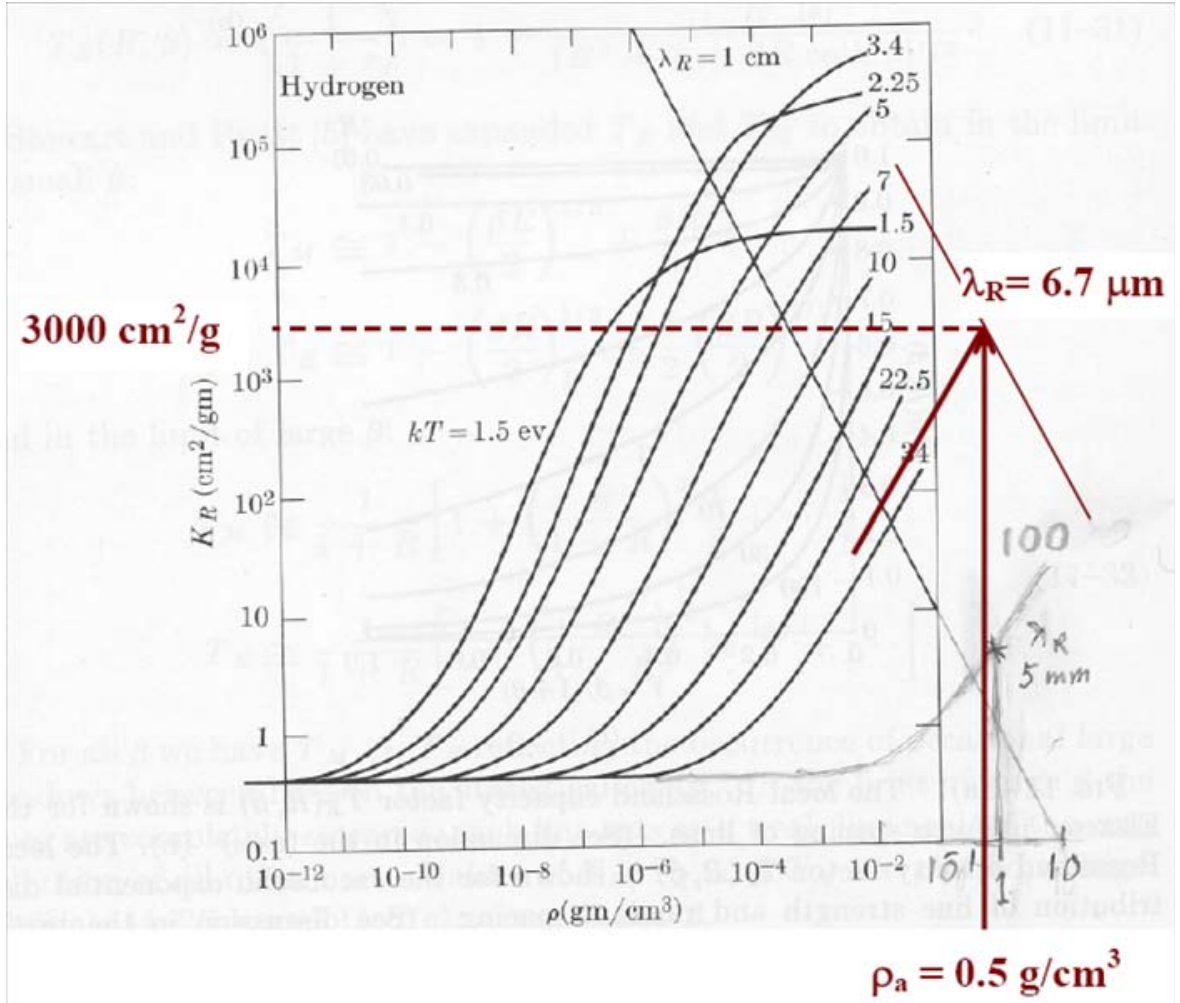

Figure 11: Opacity of $\mathrm{H} 2$ ablators are optically thick (marginally) at $\sim 30 \mathrm{eV}$. Note that if local $\mathrm{T}_{\mathrm{e}}$ at the ablation front increased for any reason to $100 \mathrm{eV}$, however, this would no longer be true, and a thin $\sim 1 \mathrm{mg} / \mathrm{cm}^{2}$ plastic $\mathrm{CH}$ layer between the outer DD fuel radius and the hydrogen ablator would be required to avoid preheat of the DD. The associated black body radiation loss flux is given by

$$
\Gamma_{\mathrm{bb}}\left(\alpha, \mathrm{E}_{\mathrm{f}}\right):=5.67 \cdot 10^{-12} \cdot\left(\mathrm{T}_{\mathrm{ex}}\left(\alpha, \mathrm{E}_{\mathrm{f}}\right) \cdot 11600\right)^{4} \quad \Gamma_{\mathrm{bb}}(1.5,1)=4.91 \times 10^{10} \quad \mathrm{~W} / \mathrm{cm}^{2} \quad \text { Eq } 38
$$

For targets with $\sim 1 \mathrm{~cm}$ square ablator, this radiation loss can be neglected compared to the $100 \mathrm{TW}$ scale of the PdV work by the ablation pressure, but at $100 \mathrm{eV}$, it would become significant at $10 \%$. 
For good hydrodynamic stability during implosion, we choose a moderate in-flight aspect ratio $A_{\text {if }}=\rho_{a} R_{0} /\left(\rho_{0} \Delta \mathbf{R}_{0}\right)$ :

$$
A_{\text {if }}:=15
$$

(half the "usual" value 30), where $\rho_{a}$ is the density of the compressed in-flight shell. Typically, $\rho_{a} \sim 5 \rho_{0}$, so the initial aspect ratio $A_{\text {in }}=R_{0} / \Delta R_{0} \sim 3$. This initial aspect ratio and the known initial

$$
\mathbf{A}_{\text {in }}:=\mathbf{3} \quad \xi:=\mathbf{1}-\mathbf{A}_{\text {in }}{ }^{-1}
$$
densities and masses of hydrogen and deuterium allows us to find the outer radius $r_{a}$ of the hydrogen ablator (we can neglect the $1 \%$ DT hotspot mass in this calculation). The initial deuterium layer

$$
\rho_{\text {Do }}:=0.2 \quad \mathrm{~g} / \mathrm{cm}^{3} \text { for } D_{2} \text { pintail } \rho_{0}
$$
thickness $\delta D$ and the outer hydrogen ablator radius $r_{a}$ are solved:

Given

$$
\text { Initial guesses } \quad r_{a g}:=0.5 \quad \mathrm{~cm} \quad \delta \mathrm{D}:=0.1 \quad \mathrm{~cm}
$$

$$
\begin{aligned}
& \delta \mathrm{D}=\left[\left(\frac{3 \cdot \mathbf{M}_{\mathbf{d}}\left(\alpha, \mathbf{E}_{\mathbf{f}}\right)}{4 \cdot \pi \cdot \rho_{\mathbf{D o}}}\right)+\left(\xi \cdot \mathbf{r}_{\mathrm{ag}}\right)^{3}\right]^{0.333}-\xi \cdot \mathbf{r}_{\mathrm{ag}} \\
& \mathbf{r}_{\mathrm{ag}}=\left[\left(\frac{3 \cdot \mathbf{M}_{\mathbf{h}}\left(\alpha, \mathbf{E}_{\mathbf{f}}\right)}{4 \cdot \pi \cdot \rho_{\mathbf{H o}}}\right)+\left(\xi \cdot \mathbf{r}_{\mathrm{ag}}+\delta \mathrm{D}\right)^{3}\right]^{0.333} \\
& \operatorname{Sol}\left(\alpha, \mathbf{E}_{\mathbf{f}}\right):=\operatorname{Find}\left(\delta \mathbf{D}, \mathbf{r}_{\mathbf{a g}}\right) \\
& \delta_{\mathbf{D}}\left(\alpha, \mathbf{E}_{\mathbf{f}}\right):=\operatorname{Sol}\left(\alpha, \mathbf{E}_{\mathbf{f}}\right) \mathbf{1} \quad \text { Initial D2 layer thickness } \\
& \mathbf{r}_{\mathbf{a o}}\left(\alpha, \mathbf{E}_{\mathbf{f}}\right):=\operatorname{Sol}\left(\alpha, \mathbf{E}_{\mathbf{f}}\right) \mathbf{2} \quad \text { Initial outer } \mathrm{H} 2 \text { ablator radius }
\end{aligned}
$$

Construct an target pie-sector diagram for a power plant:

$$
\begin{aligned}
& \Delta \mathbf{R}_{\mathbf{0}}:=\frac{\mathbf{r}_{\mathbf{a o}}(1.5,1)}{\mathbf{A}_{\text {in }}} \\
& \Delta \mathbf{R}_{\mathbf{0}}=\mathbf{0 . 2 4} \\
& \rho \mathbf{r}_{\mathrm{do}}:=\mathbf{0 . 2} \cdot\left(\mathbf{r}_{\mathrm{do}}-\mathbf{r}_{\mathrm{di}}\right) \\
& \rho r_{\text {do }}=0.008 \quad \mathrm{~g} / \mathrm{cm}^{2} \\
& \rho r_{h o}:=0.1 \cdot\left(r_{a o}(1.5,1)-\mathbf{r}_{\mathbf{d o}}\right) \\
& \rho r_{h o}=0.0197 \quad \mathrm{~g} / \mathrm{cm}^{2} \\
& \mathrm{M}_{\mathrm{h}}(1.5,1)=0.092 \quad \mathrm{~g} \\
& M_{d}(1.5,1)=0.023 \quad g \\
& M_{t}(1.5,1)=0.0004 \quad g
\end{aligned}
$$$$
\mathbf{r}_{\mathbf{d i}}:=\xi \cdot \mathbf{r}_{\mathbf{a o}}(1.5,1)
$$$$
\mathbf{r}_{\mathbf{d i}}=\mathbf{0 . 4 7}
$$$$
\mathbf{r}_{\mathbf{d o}}:=\mathbf{r}_{\mathbf{d i}}+\delta_{\mathbf{D}}(\mathbf{1 . 5 , 1 )}
$$$$
\mathbf{r}_{\text {do }}=0.51
$$

Figure 12: Pie-sector diagram for the CFAR Power Plant reference case (thin $\mathrm{mg} / \mathrm{cm}^{2}$ plastic layers may be used at $D_{2}-H_{2}$ and $D_{2}$-DT interfaces).

Power Plant $\mathbf{E}_{\mathrm{f}}=1 \mathrm{MJ}$

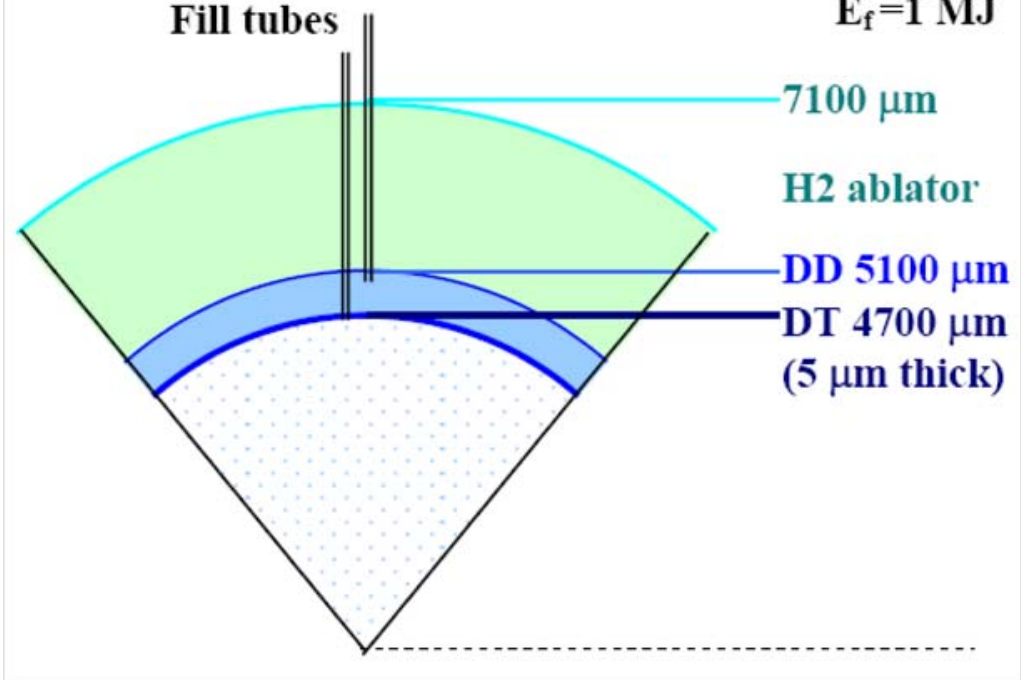

Page 16 


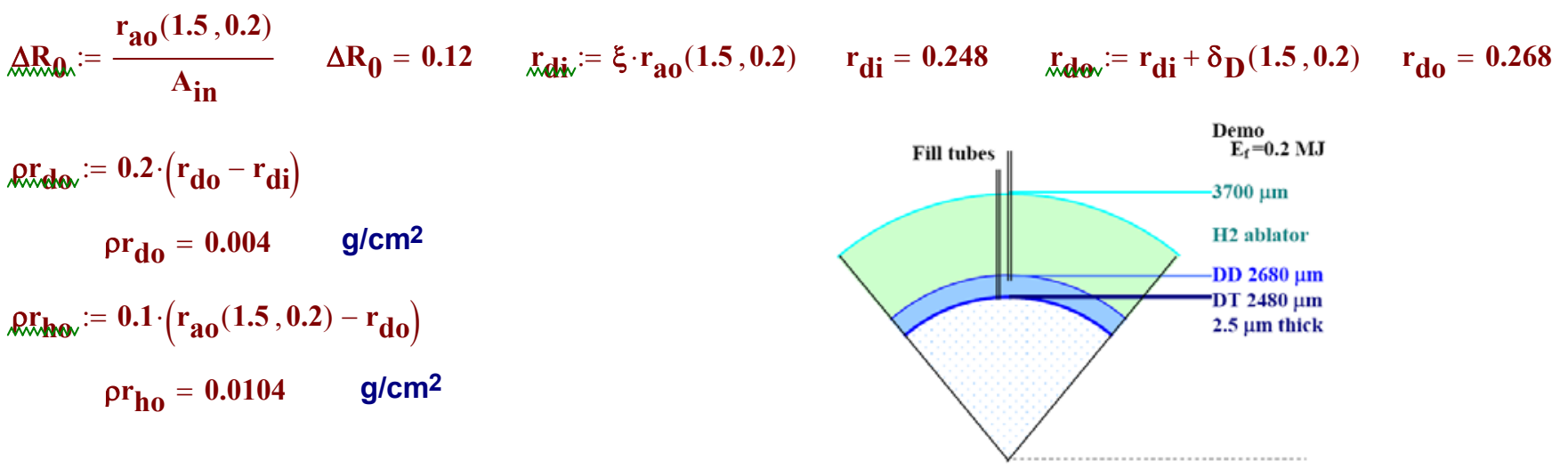

$M_{h}(1.5,0.2)=0.0134 \quad g$

$M_{d}(1.5,0.2)=0.0033 \quad g$

Figure 13: Pie-sector diagram for the CFAR DEMO reference case.

$M_{t}(1.5,0.2)=0.00006 \quad g$

(2) $3 T_{e x}$ temperature-gas energy carried by exhaust plasma implied by $u_{e x}=2 c_{T}=2\left[2 \gamma \mathrm{T} / \mathrm{m}_{\mathrm{h}}\right]^{0.5}$

(3) energy $E_{c s}$ required to pre-compress the D+DT payload to the in-flight shell density $\rho_{a}$ :

Shell compression $\quad \mathbf{E}_{\mathbf{c s}}\left(\alpha, \mathbf{E}_{\mathbf{f}}\right):=0.67 \cdot \alpha \cdot \rho_{\mathbf{a}}{ }^{0.67} \cdot\left(\mathbf{M}_{\mathbf{h}}\left(\alpha, \mathbf{E}_{\mathbf{f}}\right)+\mathbf{M}_{\mathbf{d}}\left(\alpha, \mathbf{E}_{\mathbf{f}}\right)+\mathbf{M}_{\mathbf{t}}\left(\alpha, \mathbf{E}_{\mathbf{f}}\right)\right) \quad$ (MJ)

The resulting required drive energy delivered to the ablation front is then:

$$
\begin{aligned}
\mathbf{E}_{\mathbf{d a}}\left(\alpha, \mathbf{E}_{\mathbf{f}}\right):= & \mathbf{0 . 5} \cdot 10^{-13} \cdot \mathbf{M}_{\mathbf{h}}\left(\alpha, \mathbf{E}_{\mathbf{f}}\right) \cdot \mathbf{u}_{\mathbf{e x}}\left(\alpha, \mathbf{E}_{\mathbf{f}}\right)^{2} \ldots \\
& +\left(13.6 \cdot 2+3 \cdot \mathbf{T}_{\mathbf{e x}}\left(\alpha, \mathbf{E}_{\mathbf{f}}\right)\right) \cdot 1.6 \cdot 10^{-25} \cdot \mathbf{M}_{\mathbf{h}}\left(\alpha, \mathbf{E}_{\mathbf{f}}\right) \cdot \mathbf{m}_{\mathbf{h}}{ }^{-1} \ldots \\
& +\mathbf{E}_{\mathbf{c s}}\left(\alpha, \mathbf{E}_{\mathbf{f}}\right)
\end{aligned}
$$

We can define an ion direct drive capsule implosion efficiency that takes these losses into account:

$$
\eta_{\mathbf{c}}\left(\alpha, \mathbf{E}_{\mathbf{f}}\right):=\mathbf{E}_{\mathbf{f}} \cdot \mathbf{E}_{\mathbf{d a}}\left(\alpha, \mathbf{E}_{\mathbf{f}}\right)^{-1}
$$

However, this $\eta_{c}$ is still less than the overall drive efficiency $\eta_{d f}$ because of parasitic ion beam losses by $\mathrm{dE} / \mathrm{dx}$ on outgoing ablation plasma which we will calculate later on in this model. The reason for including the latter losses separately (not in the definition of $\eta_{c}$ ) is because the parasitic beam losses are less fundamental, depending more on the selected beam ion species (Bragg peak profiles) used and on the beam illumination geometry (including 2-D effects). The fundamental upper limit to direct drive gain based on power deposited usefully at the ablation front (capsule gain, if you will) is

$$
\mathbf{G}_{\mathbf{c}}\left(\alpha, \mathbf{E}_{\mathbf{f}}\right):=\eta_{\mathbf{c}}\left(\alpha, \mathbf{E}_{\mathbf{f}}\right) \cdot \mathbf{G}_{\mathbf{f}}\left(\alpha, \mathbf{E}_{\mathbf{f}}\right)
$$

Lower overall direct drive target gains $G_{t}$ will be evaluated after we include actual beam deposition profiles, but for the next table we can preview overall target gain by using a flat $24 \%$ beam-to fuel coupling. 
Table 6: Summary of model fuel assembly parameters at stagnation for T-lean fuel assemblies versus fuel energy with $T$ localized near hotspot, $D_{2}$ main fuel, $H_{2}$ ablators with $M_{0} / M_{f}=5$ (rocket efficiency $\eta r=0.65$ ), $f=0.1 \mathrm{~T}$-load parameter, and implosion adiabat $\alpha=1.5$. (Parasitic beam loss NOT included (next section).

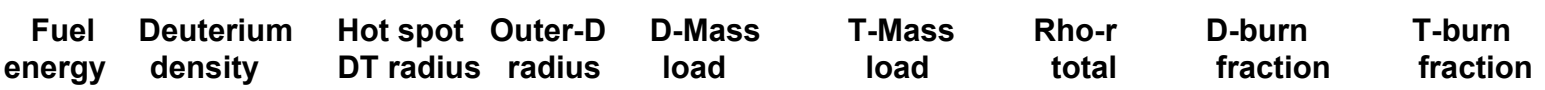

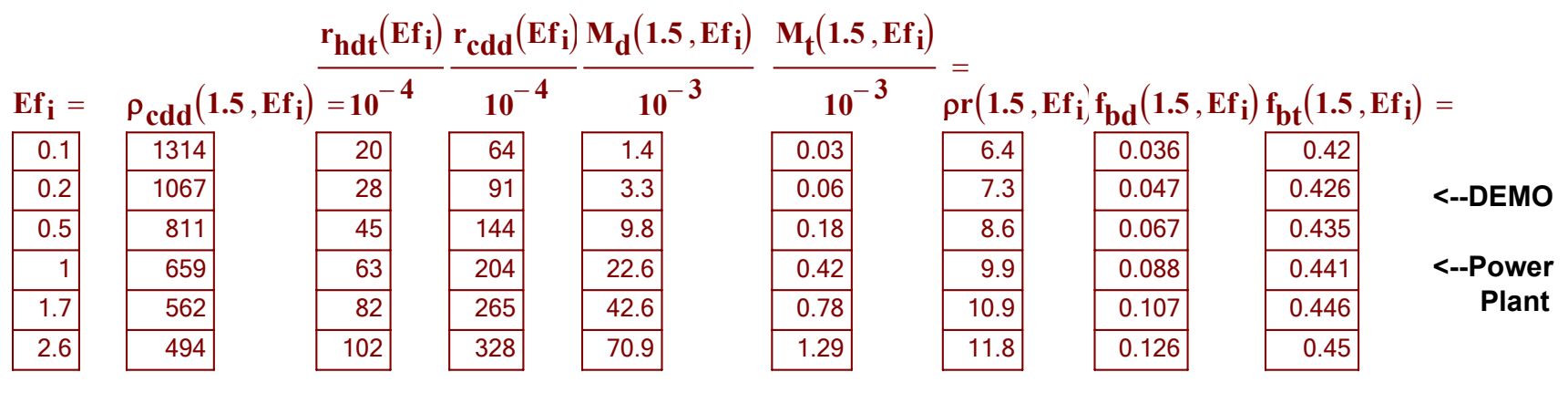

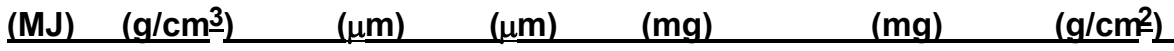

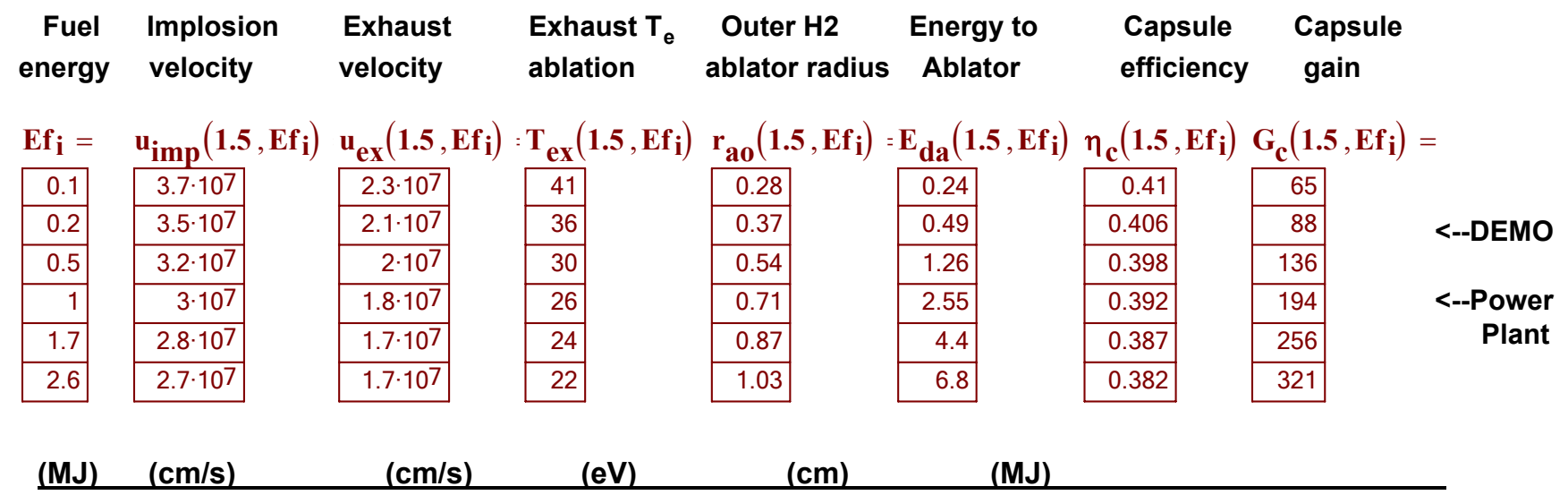

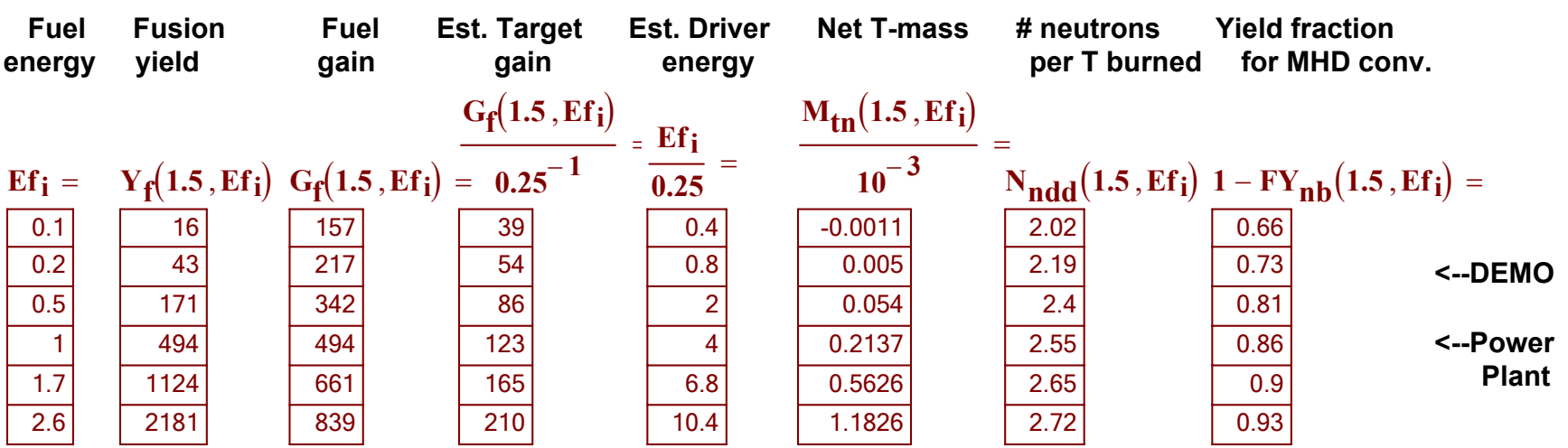

(MJ)

(MJ)

(MJ)

(mg) 


\section{lon beam range and $\mathrm{dE} / \mathrm{dx}$ profiles versus energy (and rho-r) scaling}

This MCAD model is used only as a guide to narrow the parameters to be calculated in detail with hydro codes. We'll use the ion dE/dx model used in HYDRA (Kaiser, Kerbel and Prasad), where for simplicity and the conditions given in Table 6, we can assume full ionization of the hydrogen, set:

$$
\mathbf{A}_{\mathbf{t}}:=\mathbf{1} \quad \mathrm{Z}_{\mathbf{t}}:=\mathbf{1} \quad \mathrm{Z}_{\mathrm{ta}}:=\mathbf{1}
$$

We use the same formulary as in HYDRA ion package documentation1..>

${ }^{1}$ LLNL presentation, "Implementing Ion Beams in Kull and Hydra," T. Kaiser, G. Kerbel, M. Prasad

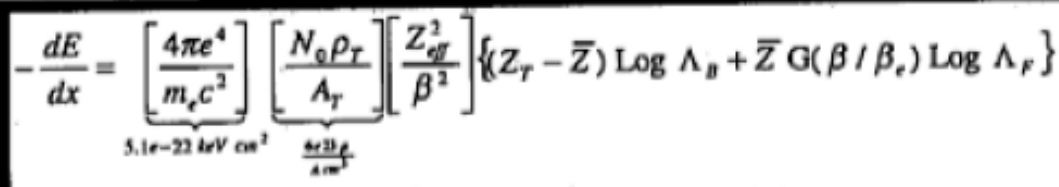

$$
\begin{aligned}
& \rho_{T}=\text { target density in } \mathrm{g} / \mathrm{cm}^{3}, A_{r}=\text { target atomic weight } \\
& Z_{r}=\text { target atomic number, } \overline{\mathrm{Z}}=\text { target ionization state } \\
& \Lambda_{b}=\frac{2 m_{t} c^{2} \beta^{2}}{\bar{l}}, \Lambda_{f}=\frac{m_{c} c^{2} \beta^{2}}{\hbar \omega_{p}}, G(x)=\operatorname{erf}(x)-x \text { erf } f^{\prime}(x)=1 \text { for } x \gg 1 \text {. } \\
& \bar{l}=\text { average ionization potential }=.01 Z_{T} \mathrm{keV} \text { (Bloch's rule) } \\
& \omega_{\rho}=\text { plasma frequency }=\sqrt{4 \pi e^{2} n_{e} / m_{e}}=56416 \sqrt{n_{e}} / \mathrm{sec} \\
& \hbar \omega_{p}=(3.7 e-14) \sqrt{n_{e}} \mathrm{keV}, n_{\mathrm{e}}=\text { electron density in } 1 / \mathrm{cm}^{3}=\bar{Z} N_{\rho} \rho_{T} / A_{T} \\
& \text { Ion Beam : } \beta=v / c, \gamma=\frac{1}{\sqrt{1-\beta^{2}}}=1+\frac{E}{M c^{2}} \\
& E=\text { Kinetic Energy of lon Beam in } k e V \text {, } \\
& M c^{2}=\text { Ion Beam Rest Energy }=A_{\text {laesrom }}(9.3 e 5) \mathrm{keV} \\
& m_{e} c^{2}=\text { Electron Rest Energy }=511 \mathrm{keV}
\end{aligned}
$$

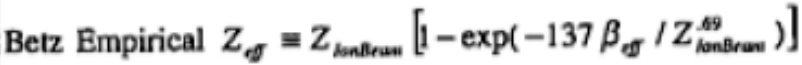

$$
\begin{aligned}
& \beta_{\sigma}^{2}=\beta^{2}+\beta_{+}^{2} \text {, with } \gamma_{\tau}=\frac{1}{\sqrt{1-\beta_{c}^{2}}}=1+\frac{k T_{\tau}}{m_{e} c^{2}}
\end{aligned}
$$

Beam nuclear Z: (nominal-we will keep ion mass dependence)

$$
\mathbf{Z}_{\mathbf{b}}:=18 \quad \mathbf{A}_{\mathbf{b}}:=40
$$

(Argon)

$$
\begin{aligned}
& M_{p}:=1.67 \cdot 10^{-27} \\
& \text { (kg), the rest mass of a proton, } \\
& \text { e }:=1.6 \cdot 10^{-19} \\
& \text { (C) electron charge, } \\
& \mathrm{c}:=\mathbf{3} \cdot \mathbf{1 0} 8 \\
& (\mathrm{~m} / \mathrm{s}) \text { the speed of light, } \\
& \mathrm{m}_{\mathrm{e}}:=9.1 \cdot 10^{-31} \\
& \varepsilon_{0}:=8.85 \cdot 10^{-12} \\
& \text { Vacuum permittivity (Farads/m), } \\
& I_{0}:=3.1 \cdot 10^{7} \\
& \mu_{0}:=4 \cdot \pi \cdot 10^{-7} \\
& \text { Vacuum permeability (Henrys/m) }
\end{aligned}
$$

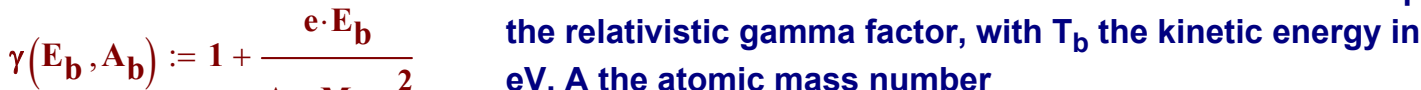
$\mathbf{A}_{\mathbf{b}} \cdot \mathbf{M}_{\mathbf{p}} \cdot \mathbf{c}^{2}$

$\beta\left(E_{b}, A_{b}\right):=\sqrt{1-\gamma\left(E_{b}, A_{b}\right)^{-2}}$ the ion velocity normalized to $c . \quad \beta\left(2 \cdot 10^{8}, A_{b}\right)=0.1$

$\gamma_{\mathbf{e}}\left(\mathbf{T}_{\mathbf{e}}\right):=1+\frac{\mathbf{e} \cdot \mathbf{T}_{\mathbf{e}}}{\mathbf{m}_{\mathbf{e}} \cdot \mathbf{c}^{\mathbf{2}}}$

Relativistic gamma for electrons of temperature $\mathrm{Te}(\mathrm{eV})$ 
$\beta_{\mathbf{e}}\left(\mathbf{T}_{\mathbf{e}}\right):=\sqrt{1-\gamma_{\mathbf{e}}\left(\mathbf{T}_{\mathbf{e}}\right)^{-2}}$

$\mathbf{T}_{\mathbf{e}}:=\mathbf{3 0}$

$\beta_{\mathrm{e}^{(30)}}=\mathbf{0 . 0 1 1}$

Eq 50

$\beta_{\text {eff }}\left(\mathbf{E}_{\mathbf{b}}, \mathbf{T}_{\mathbf{e}}, \mathbf{A}_{\mathbf{b}}\right):=\left(\beta\left(\mathbf{E}_{\mathbf{b}}, \mathbf{A}_{\mathbf{b}}\right)^{2}+\beta_{\mathbf{e}}\left(\mathbf{T}_{\mathbf{e}}\right)^{2}\right)^{0.5}$

Eq 51

The ion beam effective charge while slowing down in the ablator (Betz empirical formula)

$$
\begin{aligned}
& \mathbf{Z}_{\text {eff }}\left(\mathbf{E}_{\mathbf{b}}, \mathbf{T}_{\mathbf{e}}, \mathbf{A}_{\mathbf{b}}, \mathbf{Z}_{\mathbf{b}}\right):=\mathbf{Z}_{\mathbf{b}} \cdot\left(1-\exp \left(-137 \cdot \beta_{\text {eff }}\left(\mathbf{E}_{\mathbf{b}}, \mathbf{T}_{\mathbf{e}}, \mathbf{A}_{\mathbf{b}}\right) \cdot \mathbf{Z}_{\mathbf{b}}^{-0.69}\right)\right) \\
& G_{\mathbf{v}}(\mathbf{x}):=\operatorname{erf}(\mathbf{x})-\mathbf{x} \cdot\left(\frac{d}{d x} \operatorname{erf}(\mathbf{x})\right) \quad G_{\mathbf{v}}(0)=0 \quad G_{\mathbf{v}}(0.5)=0.08 \quad G_{\mathbf{v}}(1)=0.43 \quad G_{\mathbf{v}}(2)=0.954 \\
& \Lambda_{\mathbf{B}}\left(\mathbf{E}_{\mathbf{b}}, \mathbf{A}_{\mathbf{b}}, \mathbf{I}_{\mathbf{t}}\right):=\left(2 \cdot 511 \cdot \beta\left(\mathbf{E}_{\mathbf{b}}, \mathbf{A}_{\mathbf{b}}\right)^{2} \cdot \mathbf{I}_{\mathbf{t}}^{-1}\right)+1 \quad I_{\mathbf{t}}:=13.6 \cdot 10^{-3} \quad \mathrm{keV} \text {, ionization PE for hydrogen Eq } 53 \\
& \Lambda_{\mathbf{F}}\left(\mathbf{E}_{\mathbf{b}}, \mathbf{A}_{\mathbf{b}}, \mathbf{Z}_{\mathrm{ta}}, \rho_{\mathbf{a}}\right):=\left[511 \cdot \beta\left(\mathbf{E}_{\mathbf{b}}, \mathbf{A}_{\mathbf{b}}\right)^{2} \cdot\left[3.7 \cdot 10^{-14} \cdot \sqrt{\mathbf{Z}_{\mathrm{ta}} \cdot \rho_{\mathbf{a}} \cdot\left(1.67 \cdot 10^{-24} \cdot \mathbf{A}_{\mathbf{t}}\right)^{-1}}\right]^{-1}\right]+1
\end{aligned}
$$

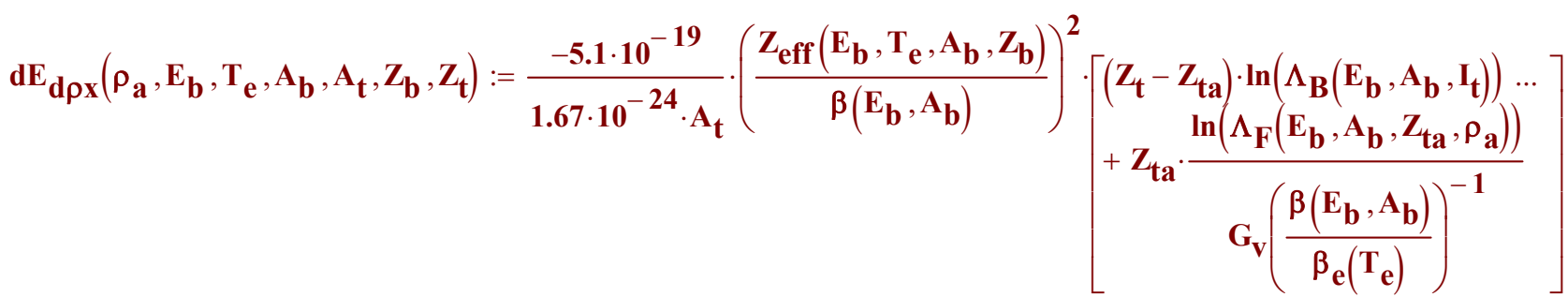

$d E / d(\rho x)$ in $\mathbf{g} / \mathrm{cm}^{2}$

Eq. 55

Consider DT first example to compare with the Barnard's HYDRA model calculations

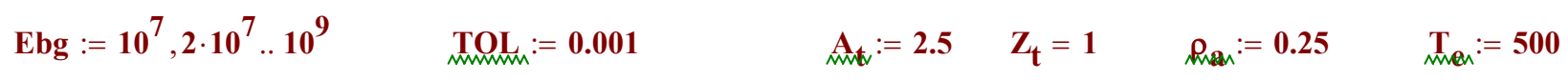

$$
\log _{10}\left(E_{o} /(d E / d \rho x)\left(g / \mathrm{cm}^{2}\right)\right.
$$

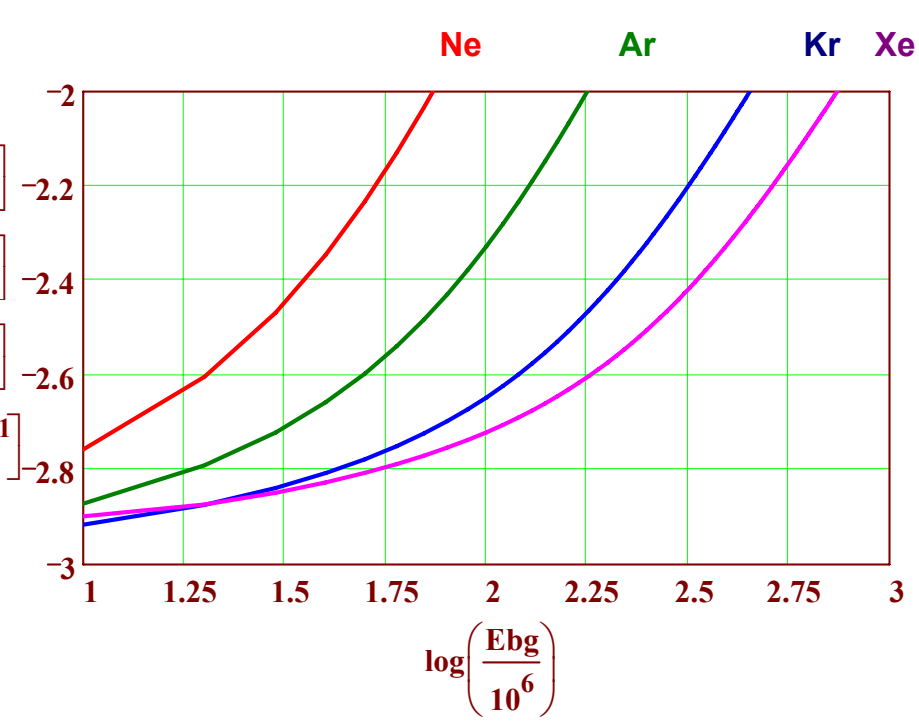

Fig. 14: Beam stopping range versus ion energy for various ions $\log _{10}\left(\mathrm{~Eb}_{\mathrm{o}}(\mathrm{Mev})\right)$ Page 20 
Now, for a more accurate representation on ion ranges, we should integrate $d E / d \rho x$ and plot the initial energies required for a specified range as a function of range, ion mass and atomic number:

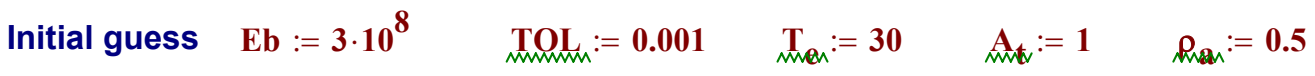

$\mathbf{E}_{\mathbf{b}}\left(\rho_{\mathbf{a}}, \rho \mathbf{\rho b}, \mathbf{T}_{\mathbf{e}}, \mathbf{A}_{\mathbf{b}}, \mathbf{A}_{\mathbf{t}}, \mathbf{Z}_{\mathbf{b}}, \mathbf{Z}_{\mathbf{t}}\right):=\operatorname{root}(\rho r b \ldots$

$+-\int_{\mathbf{E b}}^{0.02 \cdot E b} \mathbf{d E} \mathbf{d \rho x}\left(\rho_{\mathbf{a}}, \mathbf{E}, \mathbf{T}_{\mathbf{e}}, \mathbf{A}_{\mathbf{b}}, \mathbf{A}_{\mathbf{t}}, \mathbf{Z}_{\mathbf{b}}, \mathbf{Z}_{\mathbf{t}}\right)^{-1} \mathbf{d E}$

,Eb

Eq 56

$(\mathrm{eV})$

$E_{b}\left(\rho_{\mathbf{a}}, 0.01, T_{e}, 40, A_{t}, 18, Z_{t}\right)=3.916 \times 10^{8} \quad \rho r b:=0.001,0.002 \ldots 0.03$

Figure 15: Beam

range $\left(\rho r_{b}\right)$ in

$\log _{10}\left(\mathrm{~g} / \mathrm{cm}^{2}\right)$

vs beam energy

$\log _{10}(\mathrm{MeV})$,

for $\mathrm{Ar}$ and $\mathrm{Kr}$

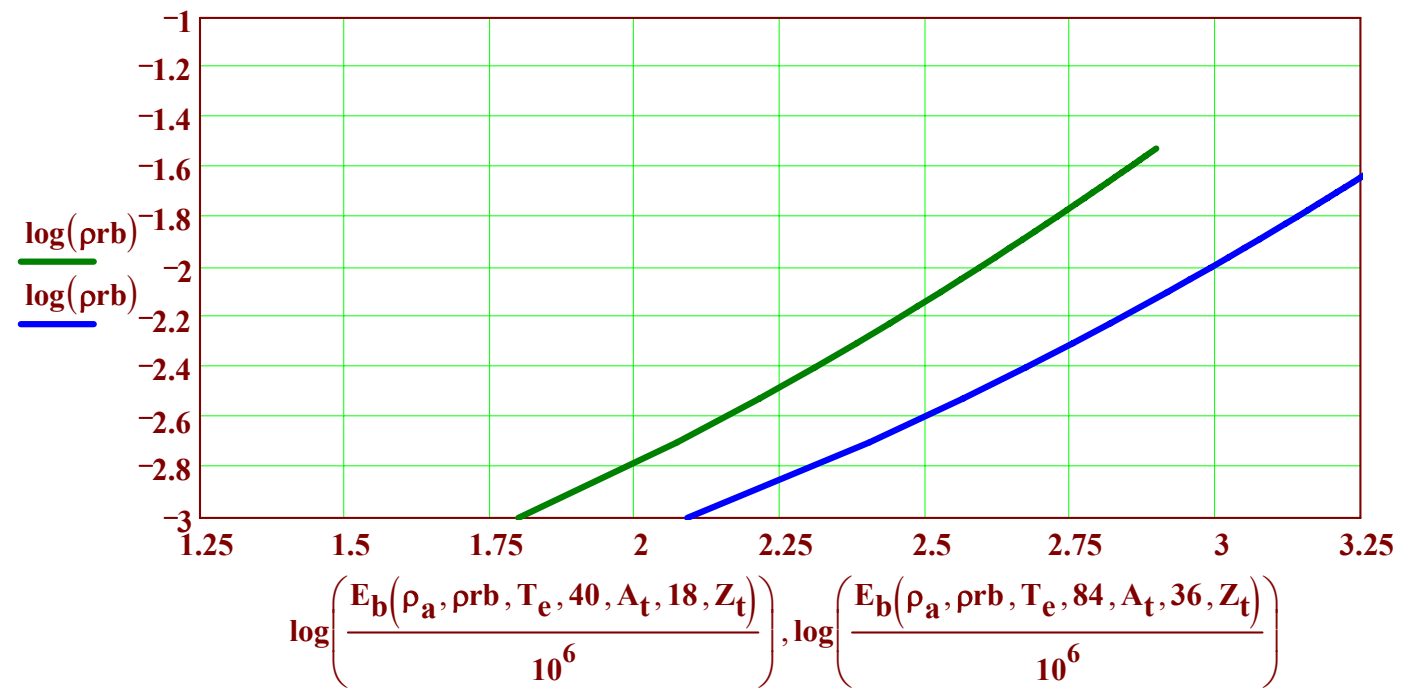

$$
\begin{aligned}
& \operatorname{Ebo}_{A r}:=\mathbf{E}_{\mathbf{b}}\left(\rho_{\mathbf{a}}, \mathbf{0 . 0 1}, \mathbf{T}_{\mathbf{e}}, 40, \mathbf{A}_{\mathbf{t}}, 18, \mathbf{Z}_{\mathbf{t}}\right) \quad \mathbf{E b o}_{\mathrm{Kr}}:=\mathbf{E}_{\mathbf{b}}\left(\rho_{\mathbf{a}}, \mathbf{0 . 0 1}, \mathbf{T}_{\mathbf{e}}, 84, \mathbf{A}_{\mathbf{t}}, 36, \mathbf{Z}_{\mathbf{t}}\right) \quad \quad \mathbf{E b o}_{\mathrm{Ar}}=3.9 \times 10^{8}
\end{aligned}
$$

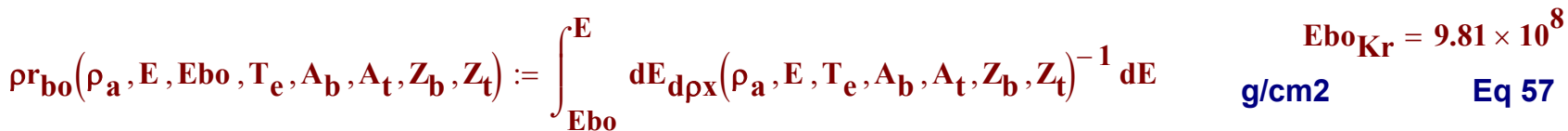

$$
\begin{aligned}
& \begin{array}{lll}
T_{\mathbf{e}}=30 & \mathbf{A}_{\mathbf{t}}=1 & \mathrm{Ebx}_{\mathrm{Ar}}:=0.01 \cdot \mathbf{E b o}_{\mathrm{Ar}}, \mathbf{0 . 0 2} \cdot \mathbf{E b o}_{\mathrm{Ar}} \cdots \mathrm{Ebo}_{\mathrm{Ar}} \\
\rho_{\mathbf{a}}=\mathbf{0 . 5} & \mathrm{Z}_{\mathrm{t}}=1 &
\end{array}
\end{aligned}
$$

Figure 16:

ion energy

vs range $\mathrm{g} / \mathrm{cm}^{2}$

for $\mathrm{Ar}$ and $\mathrm{Kr}$

Bragg peaking

(favorable to

reduce ion

loss in

ablated

plasma), is

not so

pronounced

at these low

ranges.

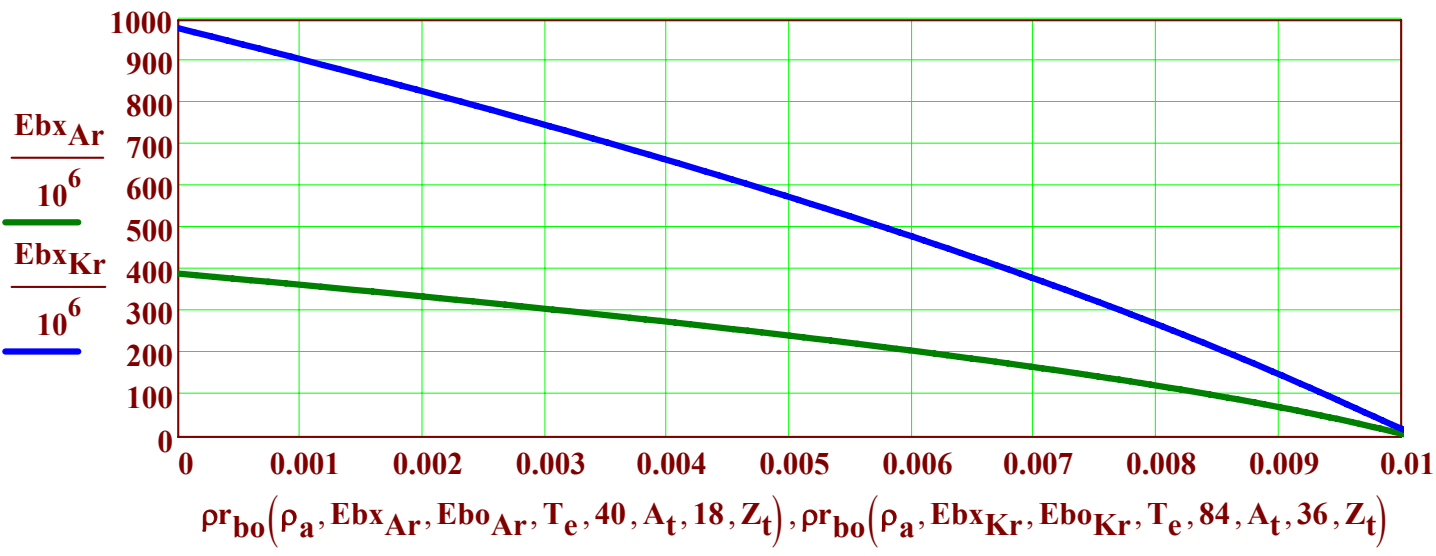

Page 21 


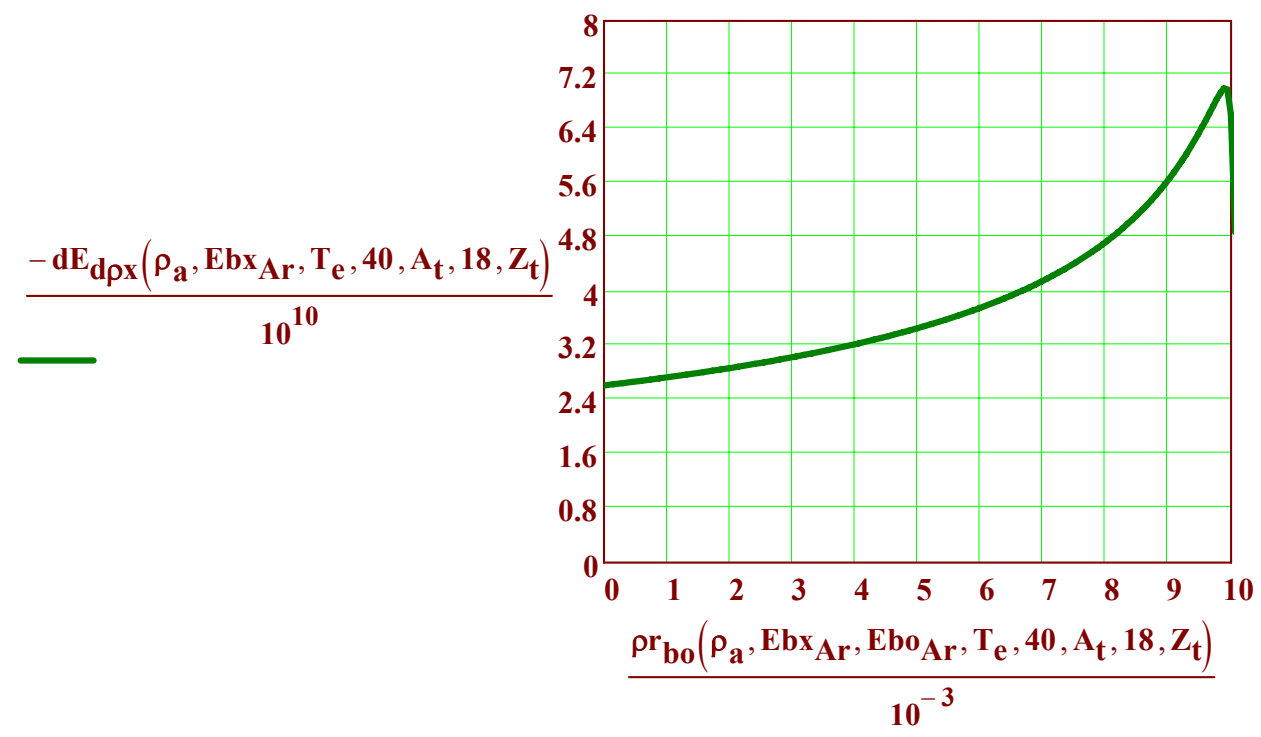

Fig. 17: Ion

dE/d $\rho x$ vs

range in

$\left(\mathrm{mg} / \mathrm{cm}^{2}\right)$ for

$\mathrm{Ar}$ (top) and

$\mathrm{Kr}$ (bottom ).

Note that

Argon Bragg

peaking is

more

pronounced

than for

Krypton for a

given range

(and even

more so

compared to

Xenon, which

would be less

peaked than

for Krypton)

$\frac{-\mathrm{dE}_{\mathbf{d} \rho \mathbf{x}}\left(\rho_{\mathbf{a}}, \mathrm{Ebx}_{\mathbf{K r}}, \mathrm{T}_{\mathbf{e}}, 84, \mathbf{A}_{\mathbf{t}}, 36, \mathbf{Z}_{\mathbf{t}}\right)}{10^{10}}$

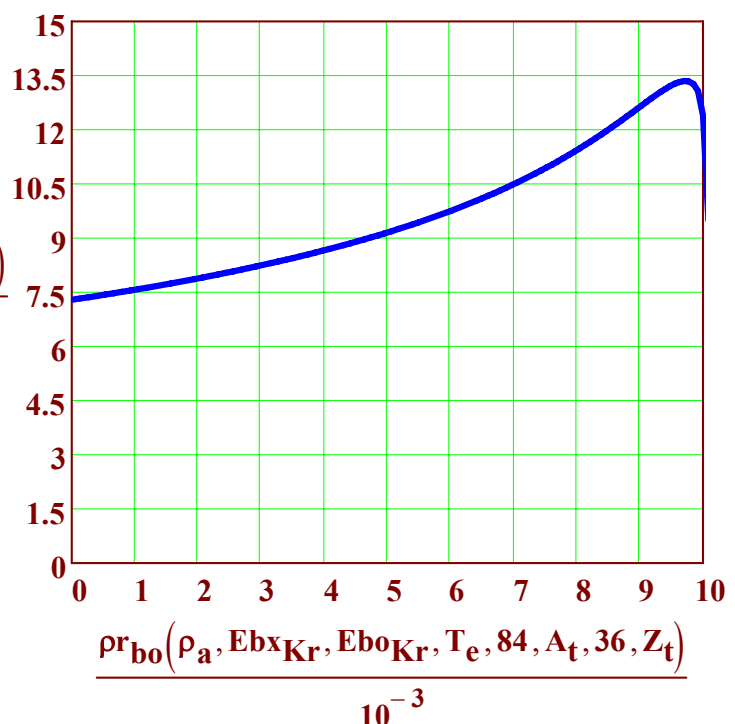

$\mathrm{mg} / \mathrm{cm}^{2}$

Now lets develop a function the ion energy lost in ablation plasma by calculating the reduced ion energy $E_{b f}$ at some specified fraction $f_{\rho r}=\rho r_{a} / \rho r_{b}$ of a given required total range $\rho r_{b}=\rho r_{a}+\delta \rho r_{h}$

Reduced ion energy $(\mathrm{eV})$ versus fraction of ion range

Ebf $:=3 \cdot 10^{8}$

$\mathbf{E}_{\mathbf{b f}}\left(\rho_{\mathbf{a}}, \rho \mathbf{\rho b}, \mathbf{f}_{\rho \mathbf{r}}, \mathbf{T}_{\mathbf{e}}, \mathbf{A}_{\mathbf{b}}, \mathbf{A}_{\mathbf{t}}, \mathbf{Z}_{\mathbf{b}}, \mathbf{Z}_{\mathbf{t}}\right):=\operatorname{root}\left(\mathbf{f}_{\rho \mathbf{r}} \cdot \rho \mathbf{r b} \ldots\right.$

$+-\int_{\mathbf{E}_{\mathbf{b}}\left(\rho_{\mathbf{a}}, \rho r \mathbf{b}, \mathbf{T}_{\mathbf{e}}, \mathbf{A}_{\mathbf{b}}, \mathbf{A}_{\mathbf{t}}, \mathbf{Z}_{\mathbf{b}}, \mathbf{Z}_{\mathbf{t}}\right)}^{\mathbf{E b f}} \mathbf{d E _ { \mathbf { d } } \mathbf { x }}\left(\rho_{\mathbf{a}}, \mathbf{E}, \mathbf{T}_{\mathbf{e}}, \mathbf{A}_{\mathbf{b}}, \mathbf{A}_{\mathbf{t}}, \mathbf{Z}_{\mathbf{b}}, \mathbf{Z}_{\mathbf{t}}\right)^{-1} \mathbf{d E}$

eV $\quad$ Eq 58 
The fraction $f_{b a}$ initial ion beam energy that is lost in the ablated plasma versus fraction of the ablation plasma $\rho$ r over total beam $\rho r_{b}$

$$
\begin{aligned}
\mathbf{f}_{\mathbf{b a}}\left(\rho_{\mathbf{a}}, \rho r b, \mathbf{f}_{\rho \mathbf{r}}, \mathbf{T}_{\mathbf{e}}, \mathbf{A}_{\mathbf{b}}, \mathbf{A}_{\mathbf{t}}, \mathbf{Z}_{\mathbf{b}}, \mathbf{Z}_{\mathbf{t}}\right):=\mathbf{1}-\frac{\mathbf{E}_{\mathbf{b f}}\left(\rho_{\mathbf{a}}, \rho r \mathbf{b}, \mathbf{f}_{\rho \mathbf{r}}, \mathbf{T}_{\mathbf{e}}, \mathbf{A}_{\mathbf{b}}, \mathbf{A}_{\mathbf{t}}, \mathbf{Z}_{\mathbf{b}}, \mathbf{Z}_{\mathbf{t}}\right)}{\mathbf{E}_{\mathbf{b}}\left(\rho_{\mathbf{a}}, \rho r b, \mathbf{T}_{\mathbf{e}}, \mathbf{A}_{\mathbf{b}}, \mathbf{A}_{\mathbf{t}}, \mathbf{Z}_{\mathbf{b}}, \mathbf{Z}_{\mathbf{t}}\right)} \\
\mathbf{f}_{\rho \mathrm{grg}}:=\mathbf{0 . 0 5 , 0 . 1 \ldots 1}
\end{aligned}
$$

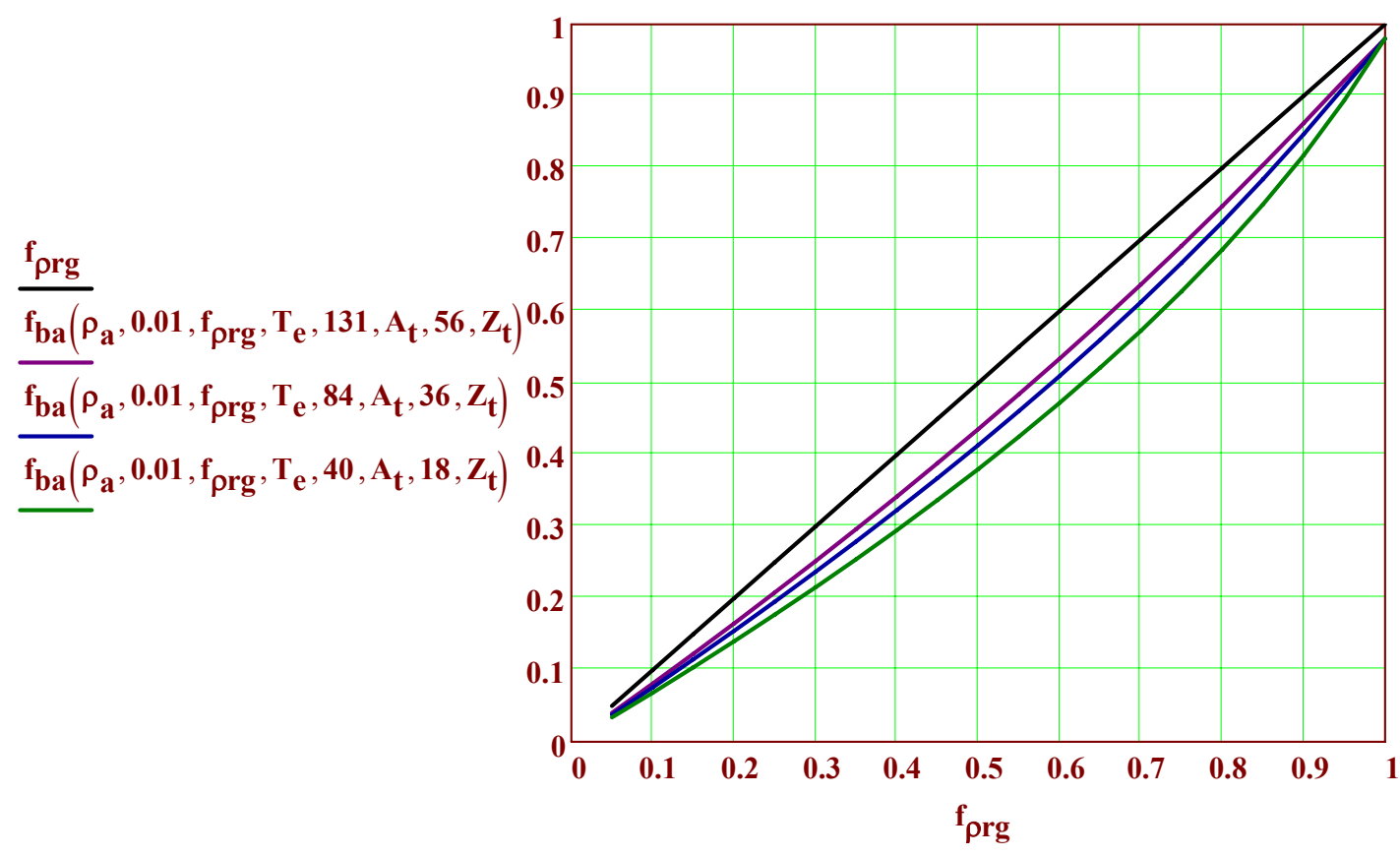

<-no Peaking effect for reference. Bragg effect $<$-for Xenon $<$-for Krypton $<$-for Argon

Figure 18. Fractions of ion beam energy loss in prior ablated plasma vs the fraction $f \rho r$ of the ablated plasma to the total ion range, for Xenon, Krypton, and Argon beams. The black line is for reference if there was no $\mathrm{dE} / \mathrm{dx}$ Bragg peaking effect at all.

Fig. 18 shows that Bragg peaking can help reduce fractional beam losses in ablated $\rho r_{a}=$ remaining ion range $\left(f_{\rho r}=0.5\right.$ ) from $50 \%$ (if there were no peaking, i.e. flat $d E / d x$ ) down to $35 \%$ for $\mathrm{A}$, and down to $39 \%$ for $\mathrm{Kr}$, and down to $41 \%$ for Xenon, due to the Bragg peaking profile effect that increases $\mathrm{dE} / \mathrm{dx}$ above some fractional range to that below. This will be useful when we calculate beam coupling efficiency in implosions with shaped beam pulses below. Since Argon gives the greatest Bragg peaking effect, we will assume Argon for the implosion calculations below. However, if accelerator issues should require it, Krypton and Xenon can be substituted for less beam space charge in the accelerator, if need be, with a small penalty in increased parasitic beam losses due to reduced Bragg peaking profiles. 


\section{Implosion Dynamics Case $A:$ at the peak of rocket efficiency $=0.65 \quad\left(M_{o} / M_{f}=5\right)$}

In the following example calculation of implosion dynamics we divide the initial hydrogen ablator into 30 Lagrangian mass layers, and calculate shell implosion dynamics by working backwards requiring the mass ablation rates satisfy the rocket equation for the chosen initial/final mass ratio. We make no attempt to calculate exact pulse shaping required for shock timing to produce the desired low $\alpha=1.5$ adiabat, that requiring actual hydro codes calculations, but we do pick a pulse shape that resembles the desired shapes we know will be required, to get the characteristics. We assume spherical symmetry, even though eventually we want to achieve the same final fuel payload conditions in two-sided asymmetric direct drive. Steve Slutz finds similar large-column density capsules can tolerate P2 asymmetries as large as $\mathbf{2 0} \%$. Our goal is to explore the implosion characteristics as a guide to later hydro code runs. By working backwards, derive the ion energies and ranges required to produce the ablation rates for the final fuel energy. We track the ablated plasma $\rho r_{a}$, increasing during the drive pulse, to estimate the beam losses incurred.

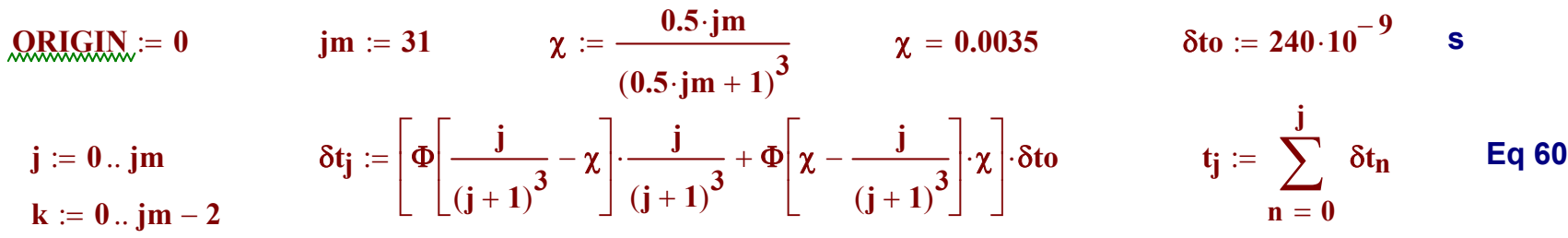

$$
\begin{aligned}
& \mathrm{jm}-1=30 \quad \mathrm{M}_{0}:=1.25 \cdot \mathrm{M}_{\mathbf{h}}(1.5,1) \quad \mathrm{M}_{\mathbf{0}}=\mathbf{0 . 1 1 4 9} \quad \mathrm{g} \quad \delta \mathrm{M}:=\frac{0.8}{\mathrm{jm}-1} \cdot \mathrm{M}_{\mathbf{0}} \\
& (\mathbf{j m}-\mathbf{1}) \cdot \delta \mathrm{M}=\mathbf{0 . 0 9 2} \quad \mathbf{M}_{\mathbf{0}}:=\mathbf{M}_{\mathbf{0}} \quad \mathbf{0 . 8} \cdot \mathbf{M}_{\mathbf{0}}=\mathbf{0 . 0 9 2} \quad \mathrm{M}_{\mathbf{j}}:=\mathrm{M}_{\mathbf{0}}-\mathbf{j} \cdot \delta M \\
& M_{d}(1.5,1)+M_{t}(1.5,1)=0.023 \quad \delta M=3.06 \times 10^{-3} \quad 0.8 \cdot M_{0} \cdot(j m-1)^{-1}=3.06 \times 10^{-3} \\
& \text { uex }:=u_{\text {ex }}(1.5,1) \quad \text { uex }=1.83 \times 10^{7} \quad u_{j}:=\operatorname{uex} \cdot \ln \left(\frac{M_{0}}{M_{j}}\right) \quad u_{i m p}(1.5,1)=2.95 \times 10^{7} \quad M_{0}:=0 \\
& M_{\mathbf{j}}:=M_{0}-M_{j} \quad \operatorname{Eex}_{\mathbf{j}}:=0.5 \cdot 10^{-13} \cdot \mathrm{Ma}_{\mathbf{j}} \cdot \mathbf{u e x}^{2}+10^{-5} \quad \mathrm{Ep}_{\mathbf{j}}:=0.5 \cdot 10^{-13} \cdot M_{\mathbf{j}} \cdot\left(\mathbf{u}_{\mathbf{j}}\right)^{2}
\end{aligned}
$$

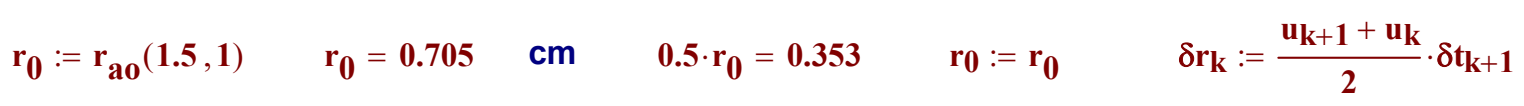

$$
\begin{aligned}
& \mathbf{r}_{\mathbf{k}}:=\mathbf{r}_{\mathbf{0}}-\sum_{\mathbf{n}=\mathbf{0}}^{\mathbf{k}}\left(\delta \mathbf{r}_{\mathbf{n}}\right) \quad \operatorname{Pex}_{\mathbf{k}}:=\frac{\left(\mathrm{Eex}_{\mathbf{k}+1}-\mathrm{Eex}_{\mathbf{k}}\right) \cdot 10^{6}}{\delta \mathbf{t}_{\mathbf{k}+1}} \sum_{\mathbf{n}=\mathbf{0}}^{\mathbf{j m}-2}\left(\operatorname{Pex}_{\mathbf{n}} \cdot \delta \mathbf{t}_{\mathbf{n}+1}\right)=1.54 \times 10^{6}
\end{aligned}
$$

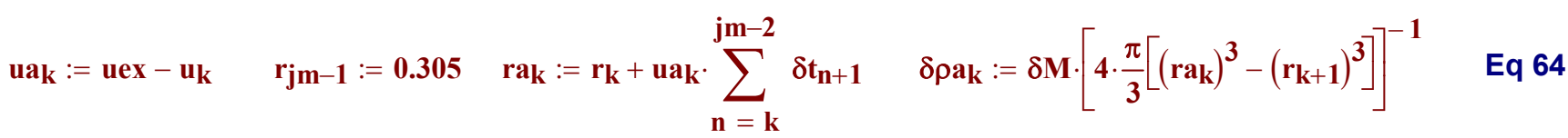

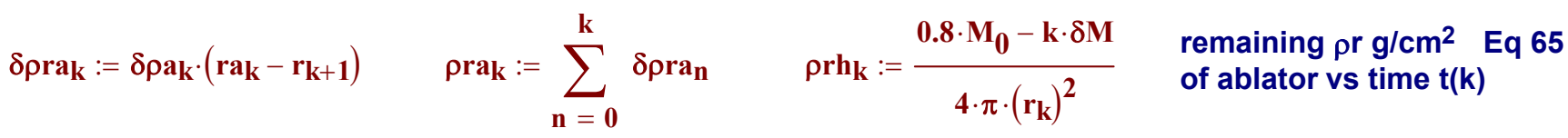

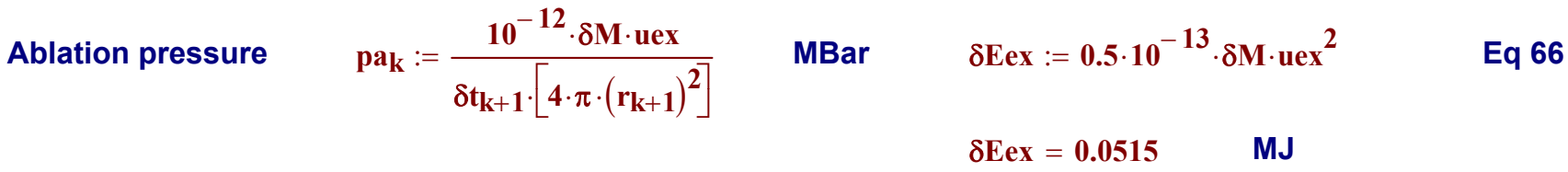


Table 7: Parameters during implosion for Case A with parasitic beam loss on uniform ablation plasma $\underset{m \sim}{m}:=0,2 . .30 \quad \operatorname{Pex}_{30}:=62 \cdot 10^{12} \quad \mathbf{r}_{30}:=0.305 \quad \operatorname{ra}_{30}:=0.3 \quad \operatorname{pra}_{30}:=27 \cdot 10^{-3} \quad \operatorname{prh}_{30}:=0 \quad \operatorname{pa}_{30}:=64$

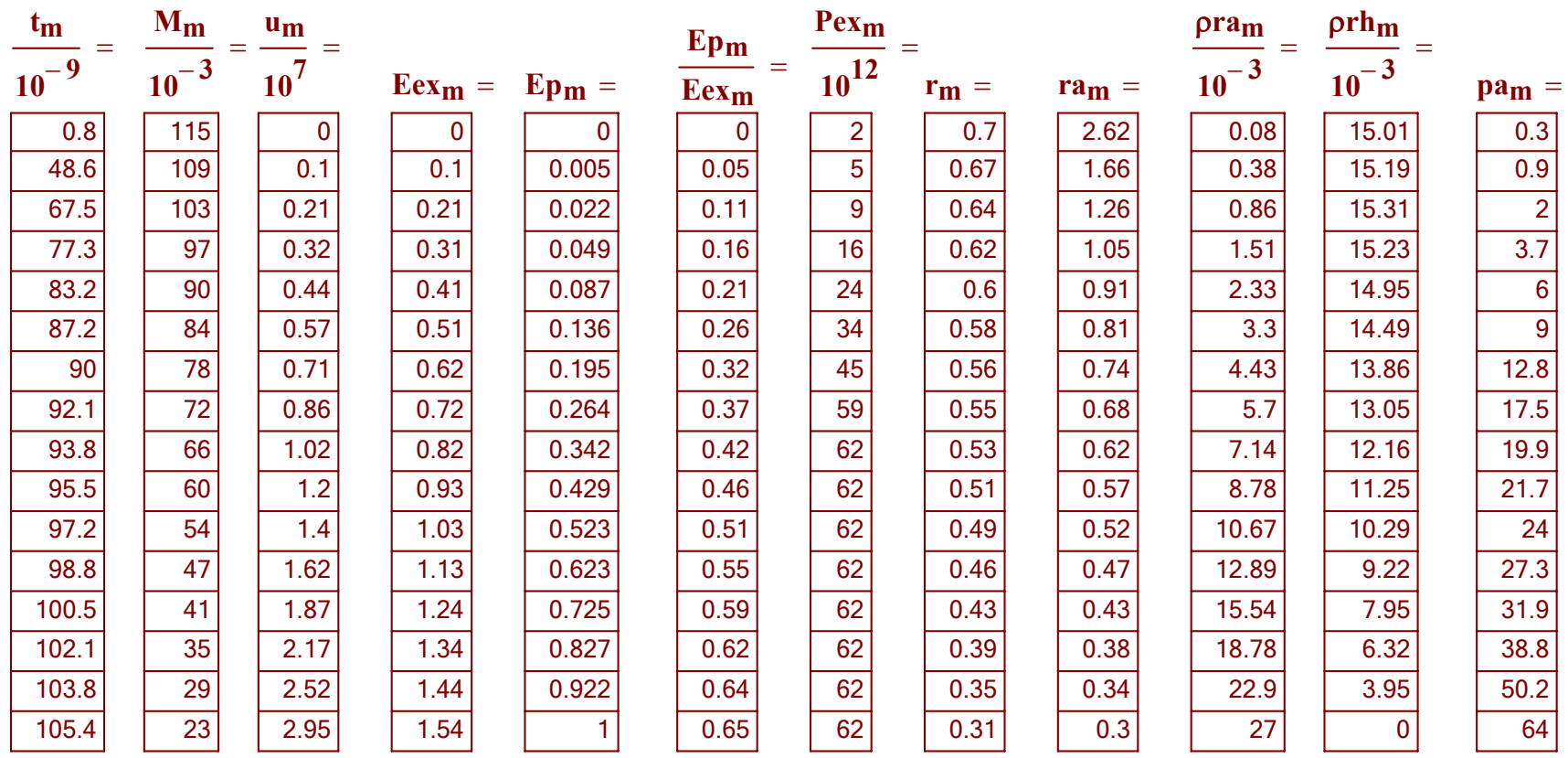

Figure 19: Case A implosion characteristics at the peak rocket efficiency $\left(M_{o} / M_{f}=5\right)$ for a fuel payload energy $E_{f}=1 M J$, final $\rho r_{f}=10 \mathrm{~g} / \mathrm{cm}^{2}$.

Ablation front radius $\mathrm{r}(\mathrm{cm})$

Exhaust energy $E_{e x}(M J)$ Ablation pressure $p_{a}$ (in100 Mbar units)

Rocket K.E. power $P_{\text {ex }}$ (in 100 TW units). (required beam power is roughly $3 \mathrm{X}$ higher)

Time axis in ns

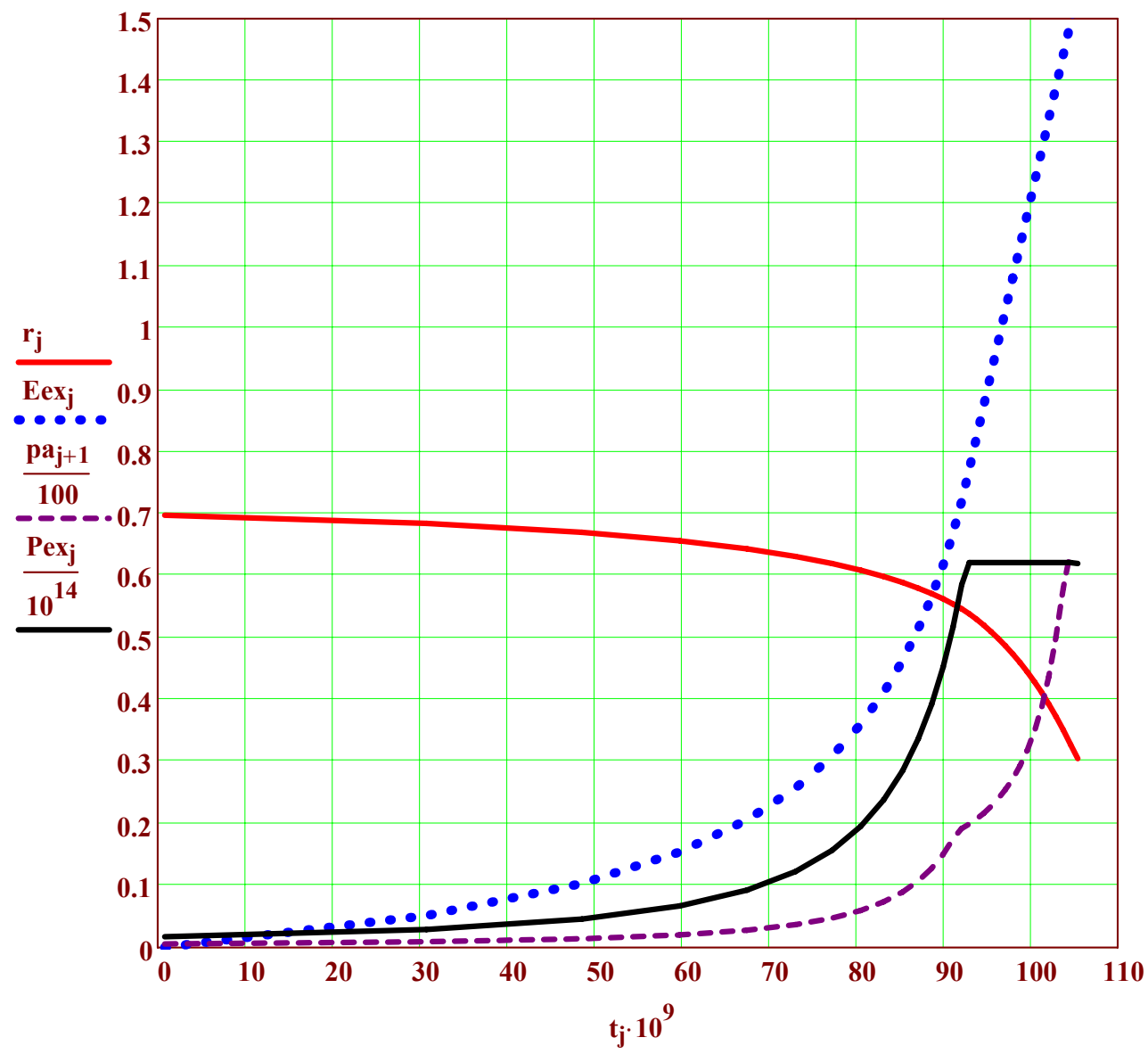

Page 25 


\section{Calculation of ion beam requirements for Case A (Fig. 19) Power Plant, $\mathrm{M}_{0} \underline{M}_{\mathrm{f}}=\underline{5}$}

$$
\delta M=0.0031 \quad \rho_{\mathrm{a}}=0.5 \quad \mathrm{~A}_{\text {if }}=15 \quad \text { uex }=1.83 \times 10^{7} \quad \mathrm{~A}_{\mathrm{b}}=40 \quad \mathrm{Z}_{\mathrm{b}}=18
$$

Given beam range penetrating ablator $\quad \delta \rho r_{b h}:=0.004 \quad \mathrm{~g} / \mathrm{cm}^{2}=0.25$ of total initial ablator $\rho r_{\text {ho }}$

Total incident required beam range $\rho \mathbf{r} \quad \rho \mathbf{r}_{\mathbf{b}}\left(\rho \mathbf{r}_{\mathbf{a}}\right):=\rho \mathbf{r}_{\mathbf{a}}+\delta \rho \mathbf{r}_{\mathbf{b h}} \quad \mathbf{g} / \mathbf{c m}^{2}$

$$
\text { Required beam range versus } \mathbf{t}(\mathbf{k}) \quad \rho \mathbf{r b}_{\mathbf{k}}:=\rho \mathbf{r}_{\mathbf{b}}\left(\rho \mathbf{r a}_{\mathbf{k}}\right) \quad \mathbf{g} / \mathrm{cm}^{2}
$$

\section{Figure 20: Case A}

Column

densities ( $\rho$ r)'s:

(g/cm² units)

Beam range in ablator $\delta \rho r_{\text {bh }}$ Ablated plasma $\rho r_{a}$ (dotted)

Total req. beam range incident

Remaining ablator $\rho r_{h}$

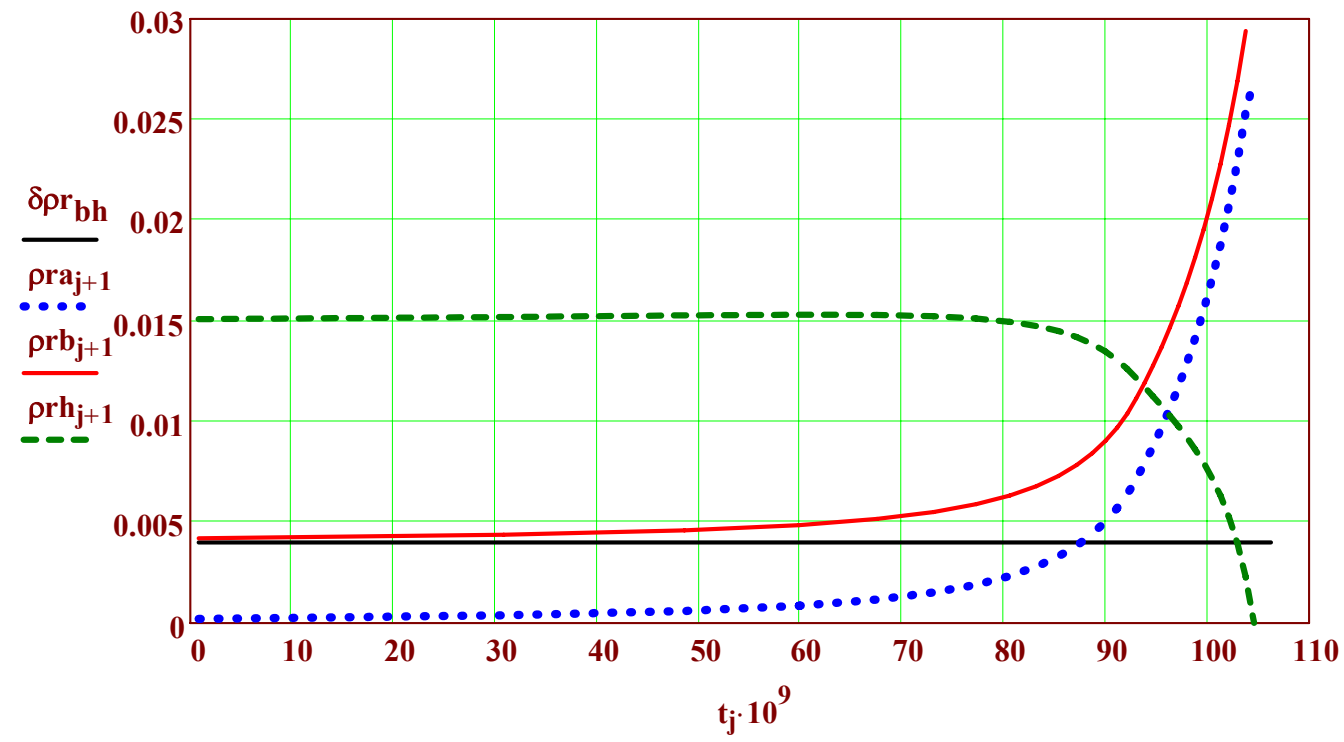

Time in ns

$$
\mathbf{f}_{\mathbf{k}}:=\frac{\rho \mathbf{r a}_{\mathbf{k}}}{\rho \mathbf{r}_{\mathbf{b}}\left(\rho \mathbf{r a}_{\mathbf{k}}\right)}
$$

Fraction of beam energy in ablated plasma

Incident beam ion K.E.

Ratio of exhaust kinetic energy to total exhaust energy

Required incident beam energy integrated up to time $t_{k}$

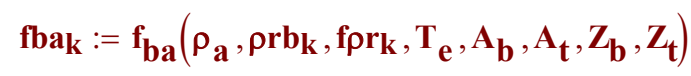

Ebok $_{\mathbf{k}}:=\mathbf{E}_{\mathbf{b f}}\left(\rho_{\mathbf{a}}, \rho \operatorname{\rho rb}_{\mathbf{k}}, 0.01, \mathbf{T}_{\mathbf{e}}, \mathbf{A}_{\mathbf{b}}, \mathbf{A}_{\mathbf{t}}, \mathbf{Z}_{\mathbf{b}}, \mathbf{Z}_{\mathbf{t}}\right) \quad \mathbf{M e V}$

$\eta_{e x}\left(\alpha, E_{f}\right):=0.5 \cdot 10^{-13} \cdot M_{h}\left(\alpha, E_{f}\right) \cdot u_{e x}\left(\alpha, E_{f}\right)^{2} \cdot E_{d a}\left(\alpha, E_{f}\right)^{-1}$

Ebinc $_{k}:=\sum_{n=0}^{k} \frac{\delta \text { Eex }}{\left(1-\text { fba }_{n}\right) \cdot \eta_{\text {ex }}(1.5,1)}$

Eq 68

Eq 69

Ebinc $_{\mathbf{j m}-2}=\mathbf{5 . 2} \quad$ (MJ) $19 \%$ beam to fuel coupling efficiency

Pbinc $_{\mathrm{k}}:=\frac{10^{-6} \cdot \delta \mathrm{Eex}}{\delta \mathrm{t}_{\mathrm{k}+1} \cdot\left(1-\mathrm{fba}_{\mathrm{k}}\right) \cdot \eta_{\mathrm{ex}}(1.5,1)} \quad$ TW $\quad$ Eq 70

Ibinc $_{k}:=$ Pbinc $_{k}\left[4 \cdot \pi \cdot\left(r_{k}\right)^{2}\right]^{-1} \quad$ TW/cm $2 \quad$ Eq 71

Page 26 


$$
A_{b}=40 \quad Z_{t}=1
$$

Argon beams

Incident beam energy $(\mathrm{GeV})$

Fractional beam loss in ablated plasma at local time $t_{k}$

Incident beam power (PW)

Incident beam intensity (PW/cm²)

Time axis in ns-->

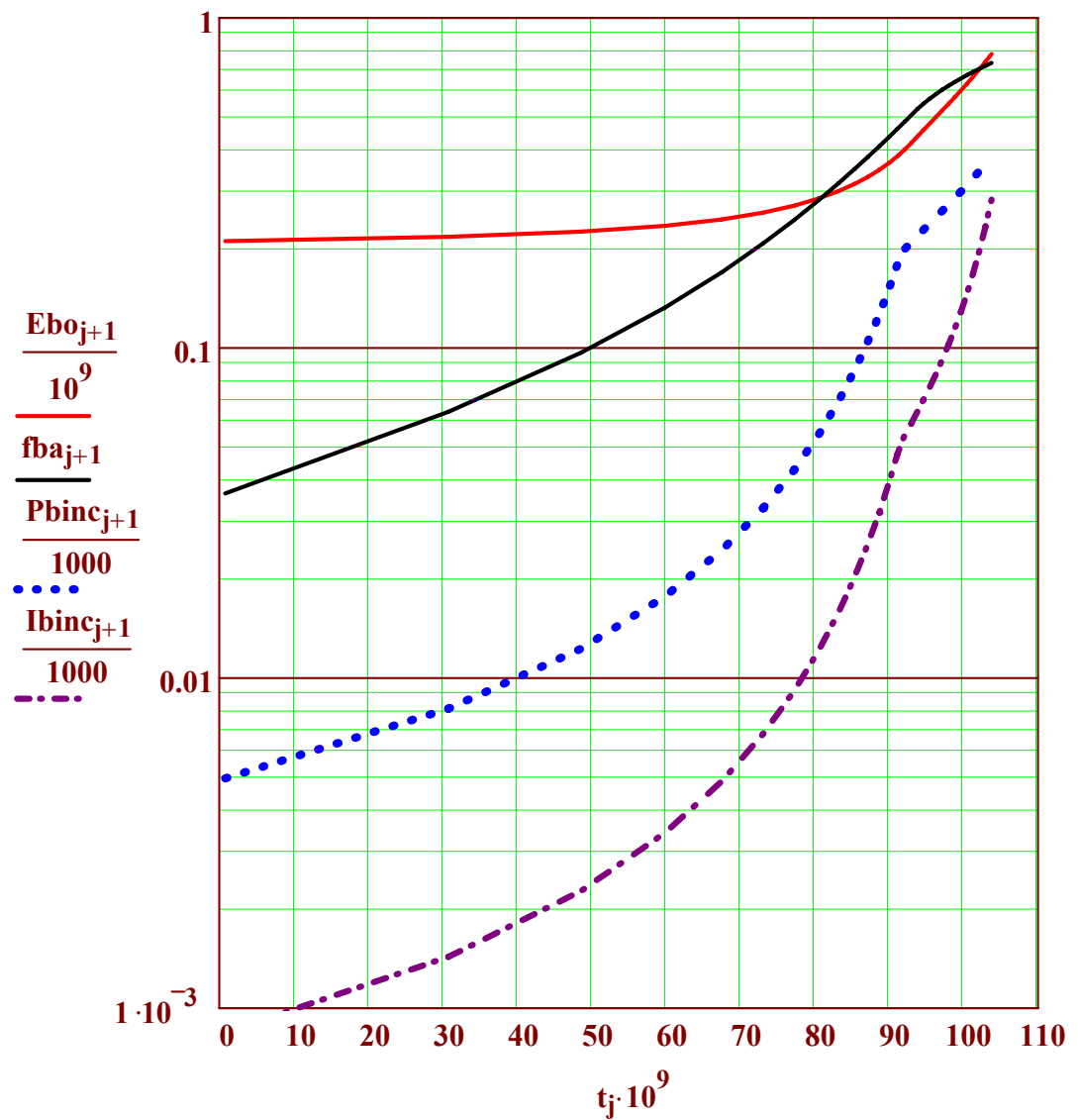

Figure. 21: Ion beam requirements for the Case A (Fig. 19) implosion example for Argon beams, $1 \mathrm{MJ}$ fuel energy at stagnation--> 5.2 $\mathrm{MJ}$ total beam deposited , 19\% total beam-to-fuel coupling efficiency.

This efficiency is still twice that of laser direct drive and 3 times more than for close-coupled indirect drive hohlraums. This case assumes zooming of the ion beam focus that follows the target ablator radius trajectory from $r_{0}=0.7 \mathrm{~cm}$ down to $r_{f}=0.35 \mathrm{~cm}$ over the $105 \mathrm{~ns}$ shaped pulse. Upstream beam time dependent focusing cab ability is included in the near term NDCX research agenda, because its required to correct for the effect of foot-to-peak velocity ramp with fixed final focus magnets on the focal spot radius. The velocity ramp is needed both to compress the accelerator bunches and here to provide the increasing ion range with time. Further requirements on time dependent upstream beam manipulations for target zooming (see work of Ed Lee in the HIF Skunkworks) will just become part of that same capability. Despite these features, the example shown in Fig. 15 is still not optimized because note the large fractional beam losses $\mathrm{f}_{\mathrm{ba}}$ in the peak power region (roughly half the incoming beam is wasted on outgoing ablation plasma despite the Bragg peak shapes shown in Fig. 12. There are three possible ways to reduce the parasitic beam losses on ablated plasma, as discussed below:

(1) A final beam energy input in a spike $5 \mathrm{~ns}$ before the last ablator burns off would couple peak power with much less than the peak ablation loss fraction, and also, by the way, would launch a final shock, that, with judicious timing, could aid ignition a la Betti-Perkins shock ignition technique.

(2) While this simple numerical model for implosions assumes spherical symmetry, the actual beam geometry we are headed for is two-sided polar as in Fig. 4, where the beam is required to deliver the same pressure to the spherical ablation front, except with a different prescription for beam intensity and ion range as a function of polar angle and time for a symmetric implosion of the fuel payload. In 2-D, the ablated plasma gets heated much more in the beam channel than with spherical beam illumination, and can expand transverse to the beam faster, thus reducing the plasma density in the beam channel. Both effects are very roughly estimated below, in such a fashion that target designers will be motivated (or outraged enough) to get the right answer with 2-D hydro code runs.

(3) Move off the rocket curve peak efficiency towards lower mass ablation fractions like laser fusion, to see if lower mass fractions and higher exhaust velocities reduce parasitic the beam loss see Case $C$.

Page 27 
(1) Late-spike method of reducing beam losses on ablation plasma. Make beam input spike up at peak intensity before end of ablation: consider inertial time for final beam spike last 5 time steps @ k=25, @ $t=101 \mathrm{~ns}, 4 \mathrm{~ns}$ before the end of ablation @ $\mathrm{t}=105 \mathrm{~ns}$ : remaining hydrogen ablator mass is $5 \delta \mathrm{M}$, and the ablation front is at $0.47 \mathrm{~cm}$ radius (about $2 / 3$ of initial radius):

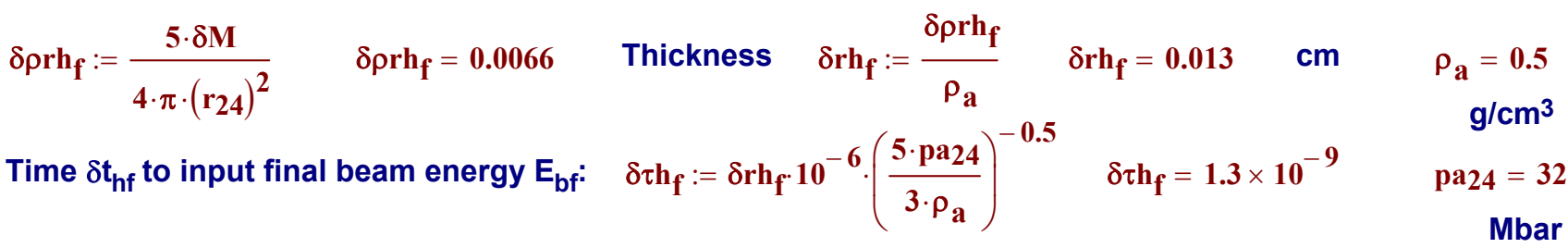

Final beam energy increment

$$
\mathrm{Eb}_{\mathrm{f}}:=5 \cdot \frac{\delta \mathrm{Eex}}{\left(1-\mathrm{fba}_{24}\right) \cdot \eta_{\mathrm{ex}}(1.5,1)} \quad \mathrm{Eb}_{\mathrm{f}}=1.23 \quad \mathrm{MJ} \quad \mathrm{fba}_{24}=0.65
$$

Total beam energy input $=\quad$ Ebinc $23_{23}+\mathbf{E b}_{\mathbf{f}}=4.76 \quad$ Ebinc $23_{23}=3.53 \quad-->$ One Petawatt final beam spike

Best case overall coupling efficiency @ $1 \mathrm{MJ}$ fuel energy= $\quad \frac{1}{\text { Ebinc } 23+\mathrm{Eb}_{\mathrm{f}}}=0.21 \quad$ with a spike

Could the spike aid Betti-Perkins-type shock ignition? It might if the timing were adjusted!

Peak pressure in the spike $\quad$ ps $:=\frac{10^{12} \cdot 10^{-11} \cdot \mathbf{E b}_{\mathrm{f}}}{\left[4 \cdot \pi \cdot\left(\mathrm{r}_{24}\right)^{2}\right] \cdot \delta \mathrm{rh}_{\mathrm{f}}} \quad \mathrm{ps}=402 \quad$ Mbar

Shock speed $\quad 10^{6} \cdot\left(\frac{5 \cdot \mathrm{ps}}{3 \cdot \rho_{\mathrm{a}}}\right)^{0.5}=3.66 \times 10^{7}$

Time to reach center

$$
\frac{\mathrm{r}_{24}}{10^{6} \cdot\left(\frac{5 \cdot \mathrm{ps}}{3 \cdot \rho_{\mathrm{a}}}\right)^{0.5}+\mathrm{u}_{\mathrm{imp}}(1.5,1)}=6.49 \times 10^{-9}
$$

Maybe a bit too late, looking at Fig. 14, but can be adjusted to have the shock aid the ignition pressure.

(2) Estimate local reduction of ablated plasma column density in the beam channel for two sided drive.

First check conditions at crossover point when $\rho_{a}>\rho r_{h}$ (see Fig. 14) $\quad t_{19}=9.63 \times 10^{-8}$

$$
\mathrm{M}_{19}=\mathbf{0 . 0 5 6 7} \quad \text { (half initial mass ablated) }
$$

In 2-D beam radius $\sim$ ablation front radius: $\quad r_{19}=0.499 \quad$ (Assume beams occupy cylinder =radius $r(t)$ )

Beam energy deposited in ablation plasma up to that point (assuming the abated plasma doesn't move)

$$
\text { Ebinc } 19=2.65_{\frac{\text { Eex19 }}{\eta_{\text {ex }}(1.5,1)}=1.62}^{\text {Ebinc19 }_{19}-\frac{\text { Eex 19 }}{\eta_{\text {ex }}(1.5,1)}=1.03} \quad \text { MJ } \quad \text { Eq } 74
$$

Now lets looks at characteristic plasma expansions versus time during the implosion

$$
\text { Beam deposition } \left.\Delta \operatorname{Eba}(\mathbf{t , k}) \quad \text { Fbac }:=0.5 \quad \Delta \text { Eba }_{\mathbf{k}}:=\text { Fbac }_{(} \cdot \text { Ebinc }_{\mathbf{k}}-\frac{\text { Eex }_{\mathbf{k}}}{\eta_{\mathrm{ex}}(1.5,1)}\right) \quad \text { MJ } \quad \text { Eq } 75
$$

Note the factor Fbac is inserted to make the net beam energy deposited smaller due to the ablated plasma expansion, it will be iterated to make the plasma expansion and beam deposition approx self consistent.

Mass of ablation plasma in the beam channel $\quad \rho r a 19=0.0097 \quad 2 \cdot \operatorname{\rho ra} 19 \cdot \pi \cdot\left(\mathbf{r}_{19}\right)^{2}=0.0152 \quad \mathrm{~g}$ 
Mean perpendicular velocity of heated ablation plasma within the channel:

$$
\begin{array}{ll}
v_{\operatorname{aperp}}\left(\Delta E_{b a}, M_{b a}\right):=10^{2} \cdot \sqrt{\frac{5 \cdot \Delta E_{b a} \cdot 10^{6}}{3 \cdot M_{b a} \cdot 10^{-3}}} & \mathrm{~cm} / \mathrm{s} \\
\operatorname{vaperp}_{k}:=v_{\text {aperp }}\left(\Delta \text { Eba }_{k}, M_{b a}\right) & t_{j}:=t_{j}
\end{array}
$$

Estimate characteristic reduction factor $f_{a r}$ for ablation plasma density in the beam current channel due to expansion caused by integrated beam heating prior to time $t(k)$ over the remaining time of the total drive pulse in the beam channel. (This characteristic method is optimistic in that expansion due to beam heating prior to time $t(k)$ doesn't apply to incremental ablation mass added after time $t(k)$, but is also pessimistic in that expansion after time $t(k)$ doesn't account for additional beam deposition heating after time $\mathbf{t}(\mathbf{k})$.

$$
\operatorname{far}_{k}:=\left(r_{k}\right)^{2} \cdot\left[r_{k}+v_{\text {aperp }}\left(\Delta \text { Eba }_{k}, M b a_{k}\right) \cdot\left(\sum_{n=k}^{j m-1} \delta t_{n+1}\right)\right]^{-2}
$$

Now lets reduce all $\rho$ ra's by the factor far: $\rho^{2}$

Fraction of ablated $\rho r$ in total beam $\rho r_{b}$

$$
\operatorname{forc}_{\mathbf{k}}:=\frac{\operatorname{\rho ra}_{\mathbf{k}} \cdot \mathbf{f a r}_{\mathbf{k}}}{\rho \mathbf{r}_{\mathbf{b}}\left(\operatorname{\rho ra}_{\mathbf{k}} \cdot \mathbf{f a r}_{\mathbf{k}}\right)}
$$

Fraction of beam energy in ablated plasma

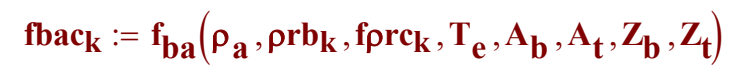

time in ns

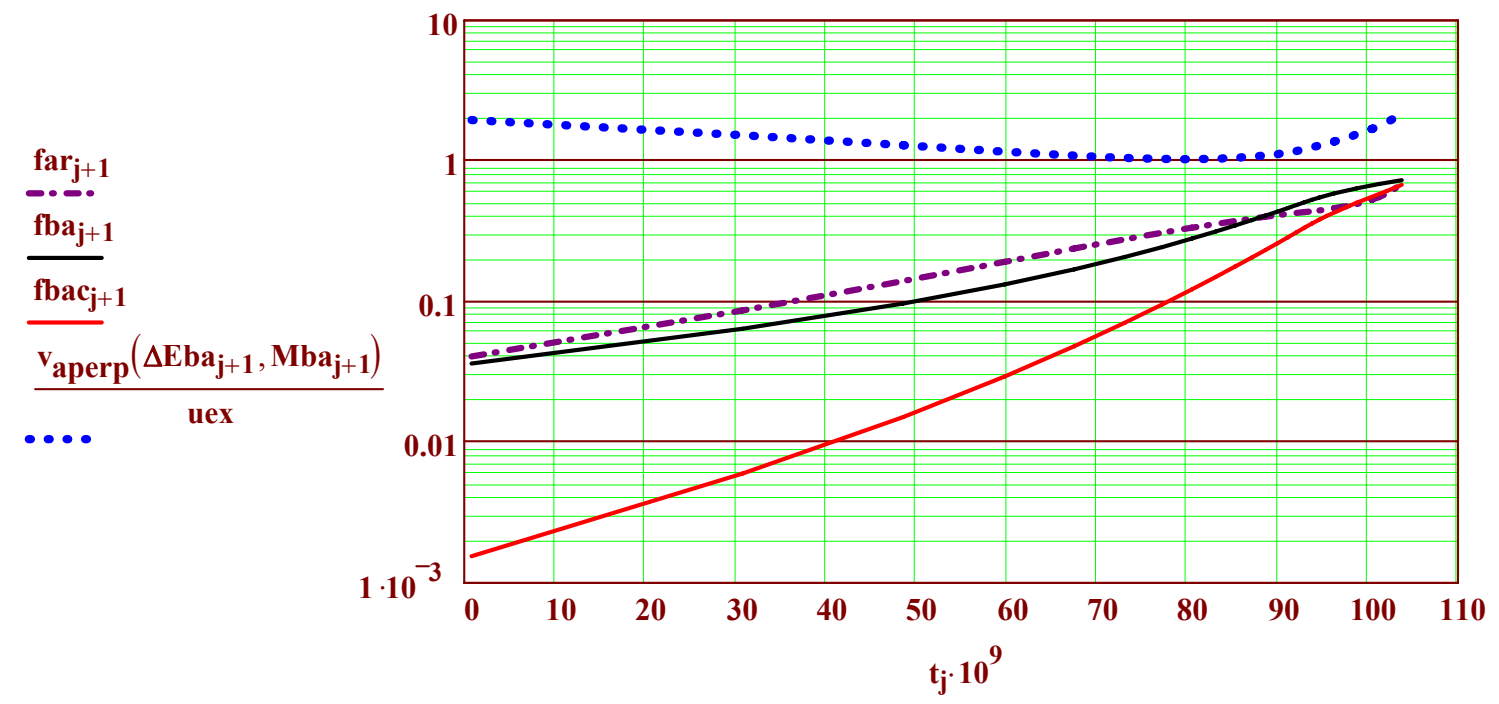

Figure 22: Ablated plasma expansion factors (column density $\rho r_{a}$ reduction factors) $f_{a r}$, and beam loss fractions without corrections for expansion $f_{\text {ba }}$ (as in Fig 21) versus time during implosion and with corrections for expansion $f_{\text {bac }}$ (red curve). Note that taking into account ablated plasma heating with expansion in the expected 2-D beam geometry reduces net beam attenuation significantly. The coefficient Fbac $=0.5$ used in the self consistent beam energy deposition was obtained from the value of fbac at the time of $96 \mathrm{~ns}$ when the remaining ablator $\rho$ rh equaled the ablated plasma $\rho$ ra in Fig. 20. Actual 2-D hydro implosion calculations will be needed to get more accurate estimates of beam loss in 2-D. Note the net beam heating is sufficient to give perpendicular plasma expansion velocities comparable to the radial uex. 
Now lets recompute Fig. 21 with the corrected (reduced) parasitic beam loss fractions $f_{b a c}$ :

Incident beam ion K.E.

Required incident beam energy integrated up to time $t_{k}$

$$
\mathbf{E b o}_{\mathbf{k}}:=\mathbf{E}_{\mathbf{b f}}\left(\rho_{\mathbf{a}}, \operatorname{\rho rb}_{\mathbf{k}}, \mathbf{0 . 0 1}, \mathbf{T}_{\mathbf{e}}, \mathbf{A}_{\mathbf{b}}, \mathbf{A}_{\mathbf{t}}, \mathbf{Z}_{\mathbf{b}}, \mathbf{Z}_{\mathbf{t}}\right) \quad \mathbf{M e V}
$$

$$
\text { Ebinc }_{k}:=\sum_{n=0}^{k} \frac{\delta \text { Eex }}{\left(1-\text { fbac }_{n}\right) \cdot \eta_{e x}(1.5,1)}
$$

$$
\begin{gathered}
\text { Ebinc }_{\mathbf{j m}-2}=4.06 \quad \eta_{\mathrm{dfA}}:=1 \cdot\left(\text { Ebinc }_{\mathrm{jm}-2}\right)^{-1} \quad \eta_{\mathrm{dfA}}=0.25 \quad<-- \text { beam to fuel coupling efficiency! } \\
\text { Required incident beam power versus } \mathrm{k} \quad \text { Pbinc }_{\mathrm{k}}:=\frac{10^{-6} \cdot \delta \mathrm{Eex}}{\delta \mathrm{t}_{\mathrm{k}+1} \cdot\left(1-\mathrm{fbac}_{\mathrm{k}}\right) \cdot \eta_{\mathrm{ex}}(1.5,1)} \quad \mathrm{TW}
\end{gathered}
$$

Incident beam intensity at radius $r_{k}$

$$
\text { Ibinck }_{\mathbf{k}}:=\text { Pbinc }_{\mathbf{k}} \cdot\left[4 \cdot \pi \cdot\left(\mathrm{r}_{\mathrm{k}}\right)^{2}\right]^{-1} \quad \text { TW/cm } 2
$$

$$
A_{b}=40 \quad Z_{t}=1
$$

\section{Argon beams}

Incident beam energy $(\mathrm{GeV})$

Fractional beam loss in ablated plasma at local time $t_{k}$ Incident beam power (PW) Incident beam intensity (PW/ $\left.\mathrm{cm}^{2}\right)$

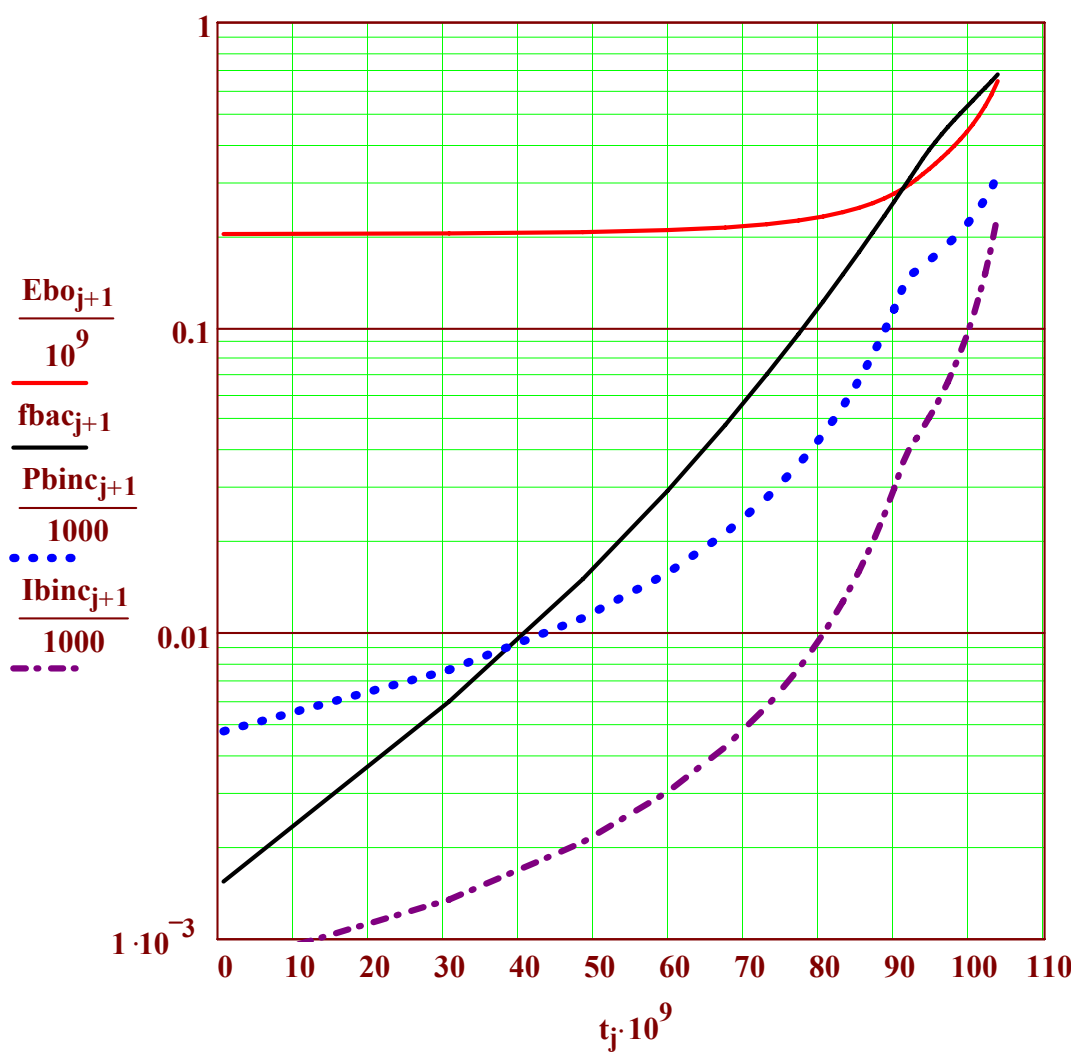

Time axis in ns-->

$$
\mathrm{t}_{\mathbf{j}} \cdot 10^{9}
$$

Fig. 23: Ion beam requirements for the Case A (Fig. 14) implosion example for Argon beams, $1 \mathrm{MJ}$ fuel energy at stagnation, corrected for 2-D expansion of ablated plasma due to beam heating $-\rightarrow 4 \mathrm{MJ}$ total beam deposited , $25 \%$ total beam-to-fuel coupling efficiency. This efficiency is $2.5 \mathrm{X}$ that of laser direct drive and 4 times more than for close-coupled indirect drive hohlraums. This case assumes zooming of the ion beam focus that follows the target ablator radius trajectory from $r_{0}=0.7 \mathrm{~cm}$ down to $r_{f}=0.35 \mathrm{~cm}$ over the $105 \mathrm{~ns}$ shaped pulse. Upstream beam time dependent focusing capability is included in the near term NDCX research agenda, because its required to correct for the effect of foot-to-peak velocity ramp with fixed final focus magnets on the focal spot radius. The velocity ramp is needed both to compress the accelerator bunches and here to provide the increasing ion range with time. Further requirements on time dependent upstream beam manipulations for target zooming (see work of Ed Lee in the HIF Skunkworks) will just become part of that same capability. 
Case B: Small DEMO at the peak of rocket efficiency $=0.65 \quad\left(M_{0} / M_{f}=5\right)$

Fuel energy $E_{f}=0.2 \mathrm{MJ}$ (nominal driver $E_{b i n c}=0.8 \mathrm{MJ} @ 20 \%$ overall coupling efficiency

Compressed D fuel density

$\rho_{\text {cdd }}(1.5,0.2)=1067 \quad \mathrm{~g} / \mathrm{cm}^{3}$

Hot spot DT radius

$\mathrm{r}_{\text {hdt }}(0.2) \cdot 10^{4}=28$ microns

Initial Outer $-D$ radius

$r_{\text {cdd }}(0.2) \cdot 10^{4}=91 \quad$ microns

D Mass load

$$
\mathrm{M}_{\mathrm{d}}(1.5,0.2) \cdot 10^{3}=3.3
$$

mg

Rho-r total at stagnation

$\operatorname{\rho r}(1.5,0.2)=7.3 \quad \mathrm{~g} / \mathrm{cm}^{2}$

D-burn fraction

$\mathbf{f}_{\text {bd }}(1.5,0.2)=0.047$

T-burn fraction

$f_{b t}(1.5,0.2)=0.426$

\section{Fusion yield}

$\mathbf{Y}_{\mathbf{f}}(1.5,0.2)=43$
Net T-mass produced

$\mathrm{M}_{\mathrm{tn}}(1.5,0.2) \cdot 10^{3}=0.005$

mg
Neutrons per T burned

$\mathrm{N}_{\text {ndd }}(1.5,0.2)=2.2$

Outer blanket shell radius

$\mathrm{r}_{\mathrm{bo}}(1.5,0.2)=4.9 \quad \mathrm{~cm}$

$$
\begin{aligned}
& \text { ORIGIN }:=0 \quad \text { im }:=31 \quad \chi_{n}:=\frac{0.5 \cdot \mathrm{jm}}{(0.5 \cdot \mathrm{jm}+1)^{3}} \quad \chi=0.0035 \quad \text { sto }:=95 \cdot 10^{-9} \quad \mathrm{~s} \\
& \begin{array}{l}
\mathbf{j}:=\mathbf{0} . . \mathbf{j m} \\
\mathbf{k}:=\mathbf{0} . . \mathbf{j m}-\mathbf{2}
\end{array} \quad \delta \mathbf{t}_{\mathbf{j}}:=\left[\Phi\left[\frac{\mathbf{j}}{(\mathbf{j}+\mathbf{1})^{\mathbf{3}}}-\chi\right] \cdot \frac{\mathbf{j}}{(\mathbf{j}+\mathbf{1})^{\mathbf{3}}}+\Phi\left[\chi-\frac{\mathbf{j}}{(\mathbf{j}+\mathbf{1})^{\mathbf{3}}}\right] \cdot \chi\right] \cdot \delta \mathbf{t o}_{\mathbf{0}} \quad \mathbf{t}_{\mathbf{j}}:=\sum_{\mathbf{n}=\mathbf{0}}^{\mathbf{j}} \delta \mathbf{t}_{\mathbf{n}} \\
& \text { jm-1 = 30 } \quad \mathrm{M}_{\mathrm{an}_{\mathrm{n}}}:=1.25 \cdot \mathrm{M}_{\mathbf{h}}(1.5,0.2) \quad \mathrm{M}_{\mathbf{0}}=0.0167 \quad \mathrm{~g} \quad \delta \mathrm{M}:=\frac{0.8}{\mathrm{jm}-1} \cdot \mathrm{M}_{\mathbf{0}} \\
& (\mathbf{j m}-\mathbf{1}) \cdot \delta \mathrm{M}=\mathbf{0 . 0 1 3} \quad \mathrm{M}_{\mathbf{0}}:=\mathrm{M}_{\mathbf{0}} \quad \mathbf{0 . 8} \cdot \mathrm{M}_{\mathbf{0}}=\mathbf{0 . 0 1 3} \quad \mathrm{M}_{\mathbf{j}}:=\mathrm{M}_{\mathbf{0}}-\mathbf{j} \cdot \delta \mathrm{M} \\
& M_{d}(1.5,0.2)+M_{t}(1.5,0.2)=3.3414 \times 10^{-3} \quad \delta M=4.46 \times 10^{-4} \quad 0.8 \cdot M_{0} \cdot(j m-1)^{-1}=4.46 \times 10^{-4} \\
& \mathrm{uex}_{\mathrm{u}}:=\mathbf{u}_{\text {ex }}(1.5,0.2) \quad \text { uex }=2.15 \times 10^{7} \quad \mathrm{u}_{\mathrm{j}}:=\operatorname{uex} \cdot \ln \left(\frac{\mathrm{M}_{0}}{\mathrm{M}_{\mathrm{j}}}\right) \quad \mathbf{u}_{\mathrm{imp}}(1.5,0.2)=3.46 \times 10^{7} \quad \operatorname{Ma}_{0}:=0 \\
& \mathrm{Ma}_{\mathbf{j}}:=\mathrm{M}_{\mathbf{0}}-\mathrm{M}_{\mathrm{j}} \quad \mathrm{Eex}_{\mathbf{j}}:=0.5 \cdot 10^{-13} \cdot \mathrm{Ma}_{\mathbf{j}} \cdot \mathbf{u e x}^{2}+10^{-5} \quad \mathrm{Ep}_{\mathrm{j}}:=0.5 \cdot 10^{-13} \cdot \mathrm{M}_{\mathrm{j}} \cdot\left(\mathrm{u}_{\mathrm{j}}\right)^{2} \\
& \mathrm{r}_{0}:=\mathrm{r}_{\mathbf{a 0}}(1.5,0.2) \quad \mathrm{r}_{0}=0.371 \quad \mathrm{~cm} \quad 0.5 \cdot \mathrm{r}_{0}=0.186 \quad \mathrm{r}_{0}:=\mathrm{r}_{\mathbf{0}} \quad \delta \mathrm{r}_{\mathrm{k}}:=\frac{\mathrm{u}_{\mathrm{k}+1}+\mathbf{u}_{\mathrm{k}}}{2} \cdot \delta \mathrm{t}_{\mathrm{k}+1} \\
& r_{k}:=r_{0}-\sum_{n=0}^{k}\left(\delta r_{n}\right) \quad \operatorname{Pex}_{k}:=\frac{\left(\operatorname{Eex}_{k}+1-E x_{k}\right) \cdot 10^{6}}{\delta t_{k+1}} \quad \sum_{n=0}^{j m-2}\left(\operatorname{Pex}_{n} \cdot \delta t_{n+1}\right)=3.09 \times 10^{5}
\end{aligned}
$$

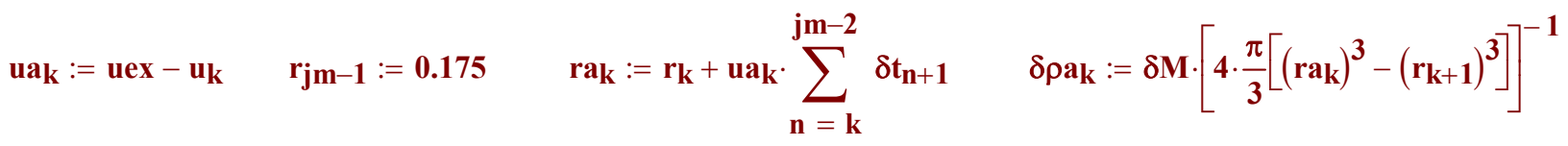

$$
\begin{aligned}
& \delta \rho \mathbf{r}_{\mathbf{k}}:=\delta \rho \mathbf{a}_{\mathbf{k}} \cdot\left(\mathbf{r a}_{\mathbf{k}}-\mathbf{r}_{\mathbf{k}+1}\right) \quad \rho \mathbf{r a}_{\mathbf{k}}:=\sum_{\mathbf{n}=\mathbf{0}}^{\mathbf{k}} \delta \rho \mathbf{r a}_{\mathbf{n}} \quad \rho \mathbf{r h}_{\mathbf{k}}:=\frac{\mathbf{0 . 8} \cdot \mathbf{M}_{\mathbf{0}}-\mathbf{k} \cdot \delta \mathbf{M}}{4 \cdot \pi \cdot\left(\mathbf{r}_{\mathbf{k}}\right)^{2}} \quad \mathbf{g} / \mathbf{c m}^{2} \\
& \text { Ablation pressure } \quad \mathrm{pa}_{\mathrm{k}}:=\frac{10^{-12} \cdot \delta \mathrm{M} \cdot \mathrm{uex}}{\delta \mathrm{t}_{\mathrm{k}+1} \cdot\left[4 \cdot \pi \cdot\left(\mathrm{r}_{\mathrm{k}+1}\right)^{2}\right]} \quad \text { MBar } \quad \begin{array}{ll}
\delta \mathrm{Eex}:=0.5 \cdot 10^{-13} \cdot \delta \mathrm{M} \cdot \mathrm{uex}^{2} \\
\text { Page 31 } & \delta \mathrm{Eex}=\mathbf{0 . 0 1 0 3}
\end{array}
\end{aligned}
$$


Table 8. Parameters during implosion for the small DEMO case B

$$
\operatorname{Pex} 30:=31 \cdot 10^{12} \quad r_{30}:=0.186 \quad \operatorname{ra}_{30}:=0.19 \quad \operatorname{pra}_{30}:=12.9 \cdot 10^{-3} \quad \operatorname{prh}_{30}:=0 \quad \operatorname{pa}_{30}:=80
$$

\begin{tabular}{|c|c|c|c|c|c|c|c|c|c|c|c|}
\hline$\frac{t_{m}}{10^{-9}}$ & $\frac{M_{m}}{10^{-3}}$ & $\frac{u_{m}}{10^{7}}$ & $\operatorname{Eex}_{\mathbf{m}}=$ & $\mathbf{E} \mathbf{p}_{\mathbf{m}}=$ & $\frac{\mathbf{E} p_{m}}{E x_{m}}$ & $\frac{P e x_{m}}{10^{12}}$ & $\mathbf{r}_{\mathbf{m}}=$ & $\mathbf{r a}_{\mathbf{m}}=$ & $\frac{\rho \mathrm{pra}_{\mathrm{m}}}{10^{-3}}$ & $\frac{\rho \mathbf{r h}_{m}}{10^{-3}}$ & $\mathbf{p a}_{\mathbf{m}}=$ \\
\hline 0.3 & 16.7 & 0 & 0 & 0 & 0 & 1 & 0.37 & 1.26 & 0.05 & 7.86 & 0.5 \\
\hline 19.2 & 15.8 & 0.12 & 0.02 & 0.0011 & 0.05 & 2 & 0.36 & 0.81 & 0.22 & 7.87 & 1.4 \\
\hline 26.7 & 14.9 & 0.24 & 0.04 & 0.0044 & 0.11 & 5 & 0.34 & 0.63 & 0.5 & 7.85 & 3.1 \\
\hline 30.6 & 14 & 0.37 & 0.06 & 0.0099 & 0.16 & 8 & 0.33 & 0.53 & 0.85 & 7.73 & 5.5 \\
\hline 32.9 & 13.1 & 0.52 & 0.08 & 0.0175 & 0.21 & 12 & 0.32 & 0.47 & 1.29 & 7.52 & 8.8 \\
\hline 34.5 & 12.3 & 0.67 & 0.1 & 0.0272 & 0.26 & 17 & 0.31 & 0.42 & 1.8 & 7.23 & 13.2 \\
\hline 35.6 & 11.4 & 0.83 & 0.12 & 0.039 & 0.32 & 23 & 0.31 & 0.39 & 2.38 & 6.85 & 18.6 \\
\hline 36.5 & 10.5 & 1 & 0.14 & 0.0528 & 0.37 & 30 & 0.3 & 0.36 & 3.03 & 6.4 & 25.3 \\
\hline 37.1 & 9.6 & 1.2 & 0.16 & 0.0685 & 0.42 & 31 & 0.29 & 0.33 & 3.75 & 5.91 & 28.5 \\
\hline 37.8 & 8.7 & 1.41 & 0.19 & 0.0858 & 0.46 & 31 & 0.28 & 0.31 & 4.55 & 5.4 & 30.6 \\
\hline 38.5 & 7.8 & 1.64 & 0.21 & 0.1046 & 0.51 & 31 & 0.27 & 0.29 & 5.46 & 4.87 & 33.4 \\
\hline 39.1 & 6.9 & 1.9 & 0.23 & 0.1246 & 0.55 & 31 & 0.26 & 0.26 & 6.5 & 4.28 & \begin{tabular}{|l|}
37.1 \\
\end{tabular} \\
\hline 39.8 & 6 & 2.2 & 0.25 & 0.1451 & 0.59 & 31 & 0.24 & 0.24 & 7.7 & 3.6 & 42.1 \\
\hline 40.4 & 5.1 & 2.54 & 0.27 & 0.1654 & 0.62 & 31 & 0.23 & 0.22 & 9.11 & 2.77 & 49.4 \\
\hline 41.1 & 4.2 & 2.95 & 0.29 & 0.1844 & 0.64 & 31 & 0.21 & 0.2 & 10.82 & 1.66 & 60.5 \\
\hline 41.7 & 3.3 & 3.46 & 0.31 & 0.2 & 0.65 & 31 & 0.19 & 0.19 & 12.9 & 0 & 80 \\
\hline
\end{tabular}

Figure 24: Case B DEMO implosion characteristics at the peak rocket efficiency $\left(M_{o} / M_{f}=5\right)$ for a fuel payload energy $E_{f}=0.2 \mathrm{MJ}$,

final $\rho r_{f}=7.3 \mathrm{~g} / \mathrm{cm}^{2}$.

Ablation front radius $\mathrm{r}(\mathrm{cm})$

Exhaust energy $E_{\text {ex }}(M J)$

Ablation pressure $p_{a}$ (in100 Mbar units)

Rocket K.E. power $P_{\text {ex }}$ (in 100 TW units). (required beam power is roughly $3 X$ higher)

Time axis in ns

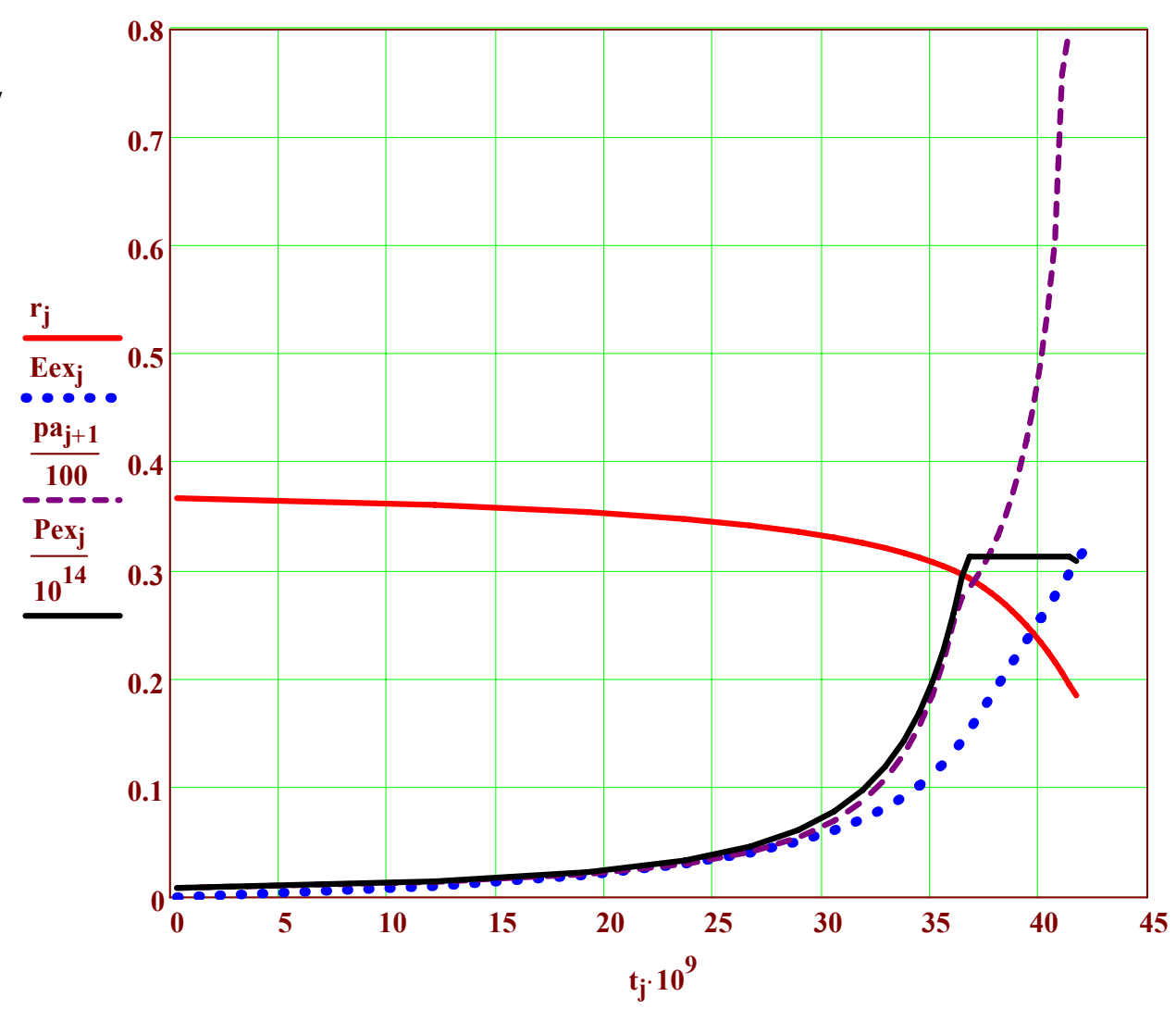

Page 32 
Given beam range penetrating ablator

$$
\delta_{\text {whrah: }}:=\mathbf{0 . 0 0 2}
$$

$\mathrm{g} / \mathrm{cm}^{2}$ (assumed constant) must be small fraction of total initial ablator $\rho r_{\text {ho }}$

$\rho \mathbf{r}_{\mathbf{b}}\left(\rho \mathbf{r}_{\mathbf{a}}\right):=\rho \mathbf{r}_{\mathbf{a}}+\delta \rho \mathbf{r}_{\mathbf{b h}} \quad \rho \mathbf{r b}_{\mathbf{k}}:=\rho \mathbf{r}_{\mathbf{b}}\left(\rho \mathbf{r a}_{\mathbf{k}}\right) \quad \mathbf{g} / \mathbf{c m}^{2}$

Fig. 25: Case B Column densities ( $\rho r)$ 's:

(g/cm² units)

Beam range in ablator $\delta \rho r_{b h}$ Ablated plasma $\rho r_{a}$ (dotted)

Total req. beam range incident

Remaining ablator $\rho r_{h}$

Time in ns

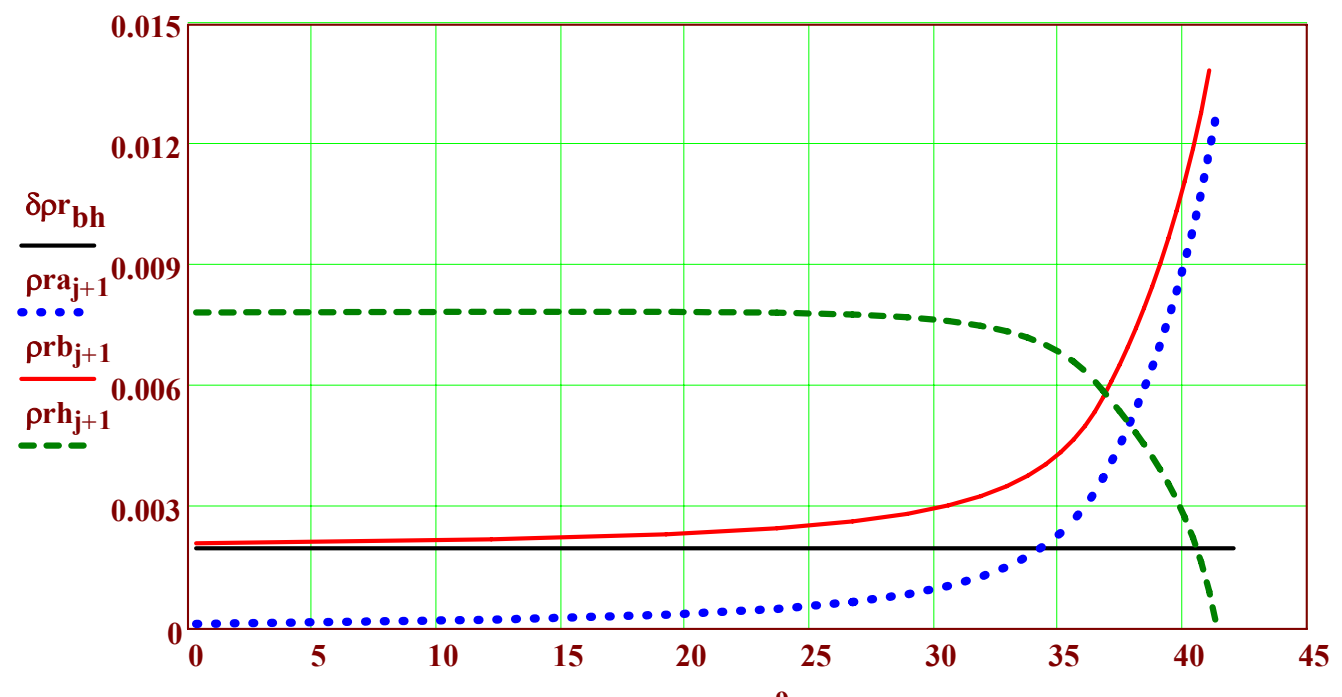
$\mathbf{t}_{\mathrm{j}} 10^{9}$

\section{Calculation of ion beam requirements for Case B-DEMO (Fig. 24)}

$$
\delta M=4.4553 \times 10^{-4} \quad \rho_{\mathrm{a}}=0.5
$$

$$
A_{\text {if }}=15
$$$$
\text { uex }=2.15 \times 10^{7}
$$

Fraction of ablated $\rho r$ in total beam $\rho r_{b}$

$$
\mathbf{f}_{\mathbf{k}}:=\frac{\rho \mathbf{r a}_{\mathbf{k}}}{\rho \mathbf{r}_{\mathbf{b}}\left(\rho \mathbf{r a}_{\mathbf{k}}\right)}
$$

Fraction of beam energy in ablated plasma

$$
\mathbf{f b a k}_{\mathbf{k}}:=\mathbf{f}_{\mathbf{b a}}\left(\rho_{\mathbf{a}}, \operatorname{\rho rb}_{\mathbf{k}}, \mathbf{f}_{\mathbf{k}}, \mathbf{T}_{\mathbf{e}}, \mathbf{A}_{\mathbf{b}}, \mathbf{A}_{\mathbf{t}}, \mathbf{Z}_{\mathbf{b}}, \mathbf{Z}_{\mathbf{t}}\right)
$$

$$
\begin{aligned}
& \text { Required incident beam energy } \\
& \text { Ebinc }_{k}:=\sum_{n=0}^{k} \frac{\delta \text { Eex }}{\left(1-\text { fba }_{n}\right) \cdot \eta_{e x}(1.5,0.2)}
\end{aligned}
$$

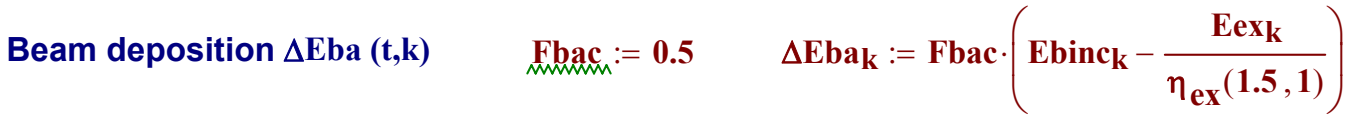

MJ

Note the factor Fbac is inserted to make the net beam energy deposited smaller due to the ablated plasma expansion, it will be iterated to make the plasma expansion and beam deposition approx self consistent.

Characteristic reduction factor $f_{a r}$ for ablation plasma density in the beam current channel due to expansion caused by integrated beam heating prior to time $t(k)$ over the remaining time of the total drive pulse in the beam channel. (This characteristic method is optimistic in that expansion due to beam heating prior to time $t(k)$ doesn't apply to incremental ablation mass added after time $t(k)$, but is also pessimistic in that expansion after time $t(k)$ doesn't account for additional beam deposition heating after time $\mathbf{t}(\mathbf{k})$. 


$$
\operatorname{Mba}_{\mathbf{k}}:=2 \cdot \operatorname{pra}_{\mathbf{k}} \cdot \pi \cdot\left(\mathbf{r}_{\mathrm{k}+1}\right)^{2} \mathbf{g}
$$

Now lets reduce all $\rho r a ' s$ by the factor far:

Fraction of ablated $\rho r$ in total beam $\rho r_{b}$

Fraction of beam energy in ablated plasma $\operatorname{far}_{k}:=\left(r_{k}\right)^{2} \cdot\left[r_{k}+v_{\operatorname{aperp}}\left(\Delta \text { Eba }_{k}, M_{b a}\right) \cdot\left(\sum_{n=k}^{j m-1} \delta t_{n+1}\right)\right]^{-2}$

$\rho \mathbf{r b}_{\mathbf{k}}:=\rho \mathbf{r}_{\mathbf{b}}\left(\operatorname{\rho ra}_{\mathbf{k}} \cdot \mathbf{f a r}_{\mathbf{k}}\right) \quad \mathbf{g} / \mathbf{c m}^{2}$

$\operatorname{forc}_{\mathbf{k}}:=\frac{\operatorname{\rho ra}_{\mathbf{k}} \cdot \mathbf{f a r}_{\mathbf{k}}}{\operatorname{\rho r}_{\mathbf{b}}\left(\operatorname{\rho ra}_{\mathbf{k}} \cdot \mathbf{f a r}_{\mathbf{k}}\right)}$

$\mathbf{f b a c}_{\mathbf{k}}:=\mathbf{f}_{\mathbf{b a}}\left(\rho_{\mathbf{a}}, \operatorname{\rho rb}_{\mathbf{k}}, \mathbf{f}_{\operatorname{coc}}, \mathbf{T}_{\mathbf{e}}, \mathbf{A}_{\mathbf{b}}, \mathbf{A}_{\mathbf{t}}, \mathbf{Z}_{\mathbf{b}}, \mathbf{Z}_{\mathbf{t}}\right)$

time in ns

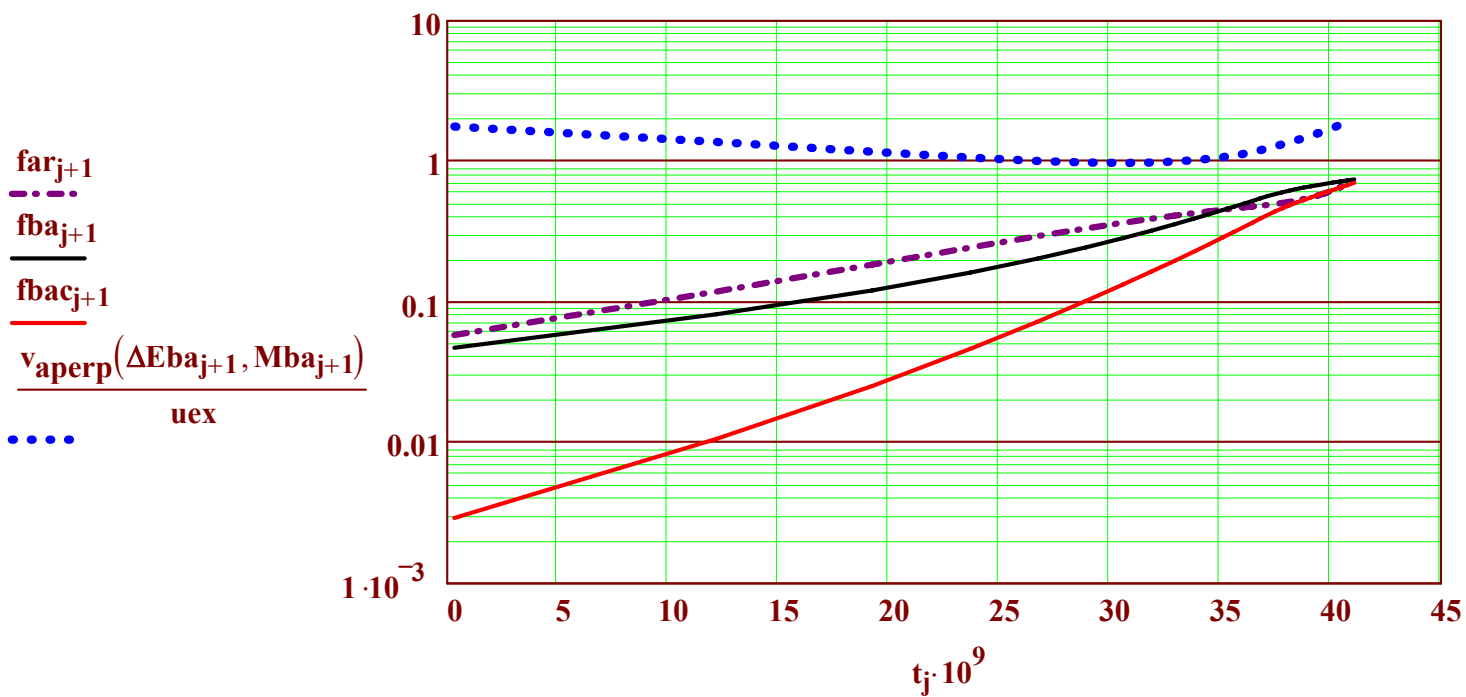

Figure 26: Ablated plasma expansion factors (column density $\rho r_{a}$ reduction factors) $f_{a r}$, and beam loss fractions without corrections for expansion $f_{b a}$ (For Case $B$ as in Fig 24) versus time during implosion and with corrections for expansion $f_{b a c}$ (red curve). Note that taking into account ablated plasma heating with expansion in the expected 2-D beam geometry reduces net beam attenuation significantly. The coefficient Fbac $=0.5$ used in the self consistent beam energy deposition was obtained from the value of fbac at the time of $38 \mathrm{~ns}$ when the remaining ablator $\rho$ rh equaled the ablated plasma $\rho_{\mathrm{a}}$ in Fig. 25.

Actual 2-D hydro implosion calculations will be needed to get more accurate estimates of beam loss in 2-D. Note the net beam heating is sufficient to give perpendicular plasma expansion velocities comparable to the radial uex.

Incident beam ion K.E.

Required incident beam energy integrated up to time $t_{k}$

$$
\mathbf{E b o}_{\mathbf{k}}:=\mathbf{E}_{\mathbf{b f}}\left(\rho_{\mathbf{a}}, \operatorname{\rho rb}_{\mathbf{k}}, \mathbf{0 . 0 1}, \mathbf{T}_{\mathbf{e}}, \mathbf{A}_{\mathbf{b}}, \mathbf{A}_{\mathbf{t}}, \mathbf{Z}_{\mathbf{b}}, \mathbf{Z}_{\mathbf{t}}\right) \quad \mathbf{M e V}
$$$$
\text { Ebinc }_{\mathrm{k}}:=\sum_{\mathrm{n}=\mathbf{0}}^{\mathrm{k}} \frac{\delta \mathrm{Eex}}{\left(1-\mathrm{fbac}_{\mathrm{n}}\right) \cdot \eta_{\mathrm{ex}}(1.5,0.2)}
$$
Ebinc $_{\mathbf{j m}-2}=\mathbf{0 . 8 5}$
$\eta_{\mathbf{d f B}}:=0.2 \cdot\left(\text { Ebinc }_{\mathbf{j m}-2}\right)^{-1}$
$\eta_{\mathrm{dfB}}=0.24$
$<--23 \%$ beam to fuel coupling efficiency for DEMO

Page 34 
Required incident beam power versus $k \quad$ Pbinc $_{k}:=\frac{10^{-6} \cdot \delta E \text { ex }}{\delta t_{k+1} \cdot\left(1-\mathrm{fbac}_{\mathrm{k}}\right) \cdot \eta_{\mathrm{ex}}(1.5,0.2)} \quad$ TW Incident beam intensity at radius $r_{k}$

$$
\text { Ibinc }_{k}:=\text { Pbinc }_{k} \cdot\left[4 \cdot \pi \cdot\left(\mathbf{r}_{\mathbf{k}}\right)^{2}\right]^{-1} \quad \mathrm{TW} / \mathrm{cm}^{2}
$$

$$
A_{b}=40 \quad Z_{t}=1
$$

\section{Argon beams}

Incident beam energy (GeV)

Fractional beam loss in ablated plasma at local time $t_{k}$ Incident beam power (PW) Incident beam intensity $\left(\mathrm{PW} / \mathrm{cm}^{2}\right)$

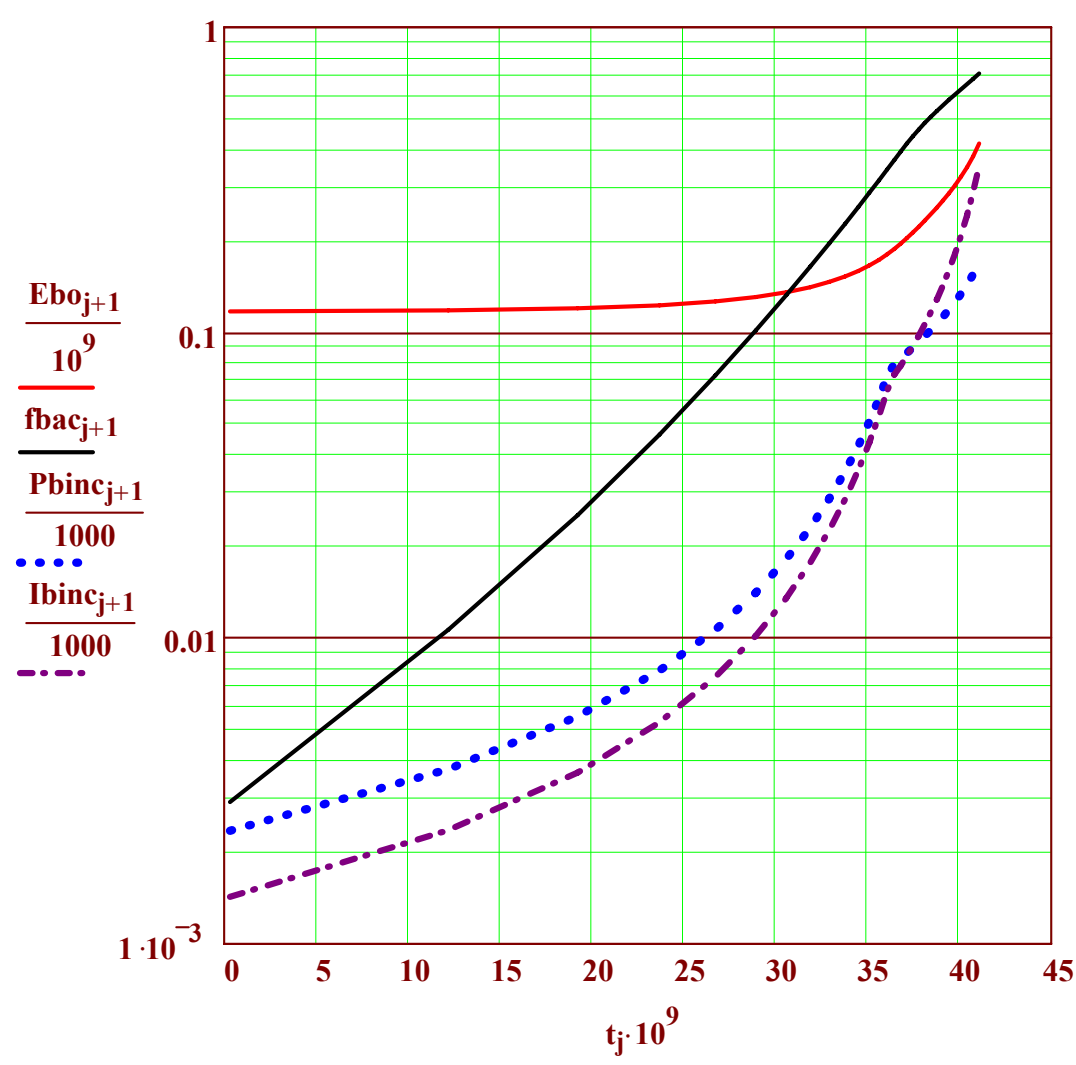

Time axis in ns-->

Figure 27. Beam requirements for the Case B -DEMO at the peak of rocket efficiency: $24 \%$ overall beam-to-fuel coupling efficiency. 
Implosion Dynamics Case C: at rocket efficiency $\sim 0.5\left(\mathrm{M}_{0} / \mathrm{M}_{\mathrm{f}}=2->\right.$ more like laser direct drive $)$ We investigate this case even though the rocket efficiency is lower (see Fig. 10) in hopes that the reduced ablator mass and higher exhaust velocities will reduce incoming ion beam losses on ablated plasma.

$$
\begin{aligned}
& \mathbf{M}_{\mathbf{h}}\left(\alpha, \mathbf{E}_{\mathbf{f}}\right):=\mathbf{M}_{\mathbf{d}}\left(\alpha, \mathbf{E}_{\mathbf{f}}\right)+\mathbf{M}_{\mathbf{t}}\left(\alpha, \mathbf{E}_{\mathbf{f}}\right) \\
& M_{h}(1.5,1)=0.023 \\
& \underset{\max }{\operatorname{un}}\left(\alpha, \mathbf{E}_{\mathbf{f}}\right):=\mathbf{u}_{\mathrm{imp}}\left(\alpha, \mathbf{E}_{\mathbf{f}}\right) \cdot \ln (2)^{-1}
\end{aligned}
$$

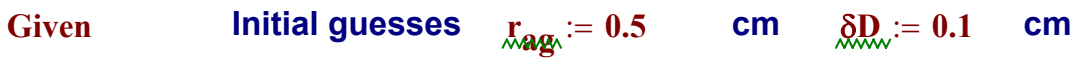

$$
\begin{aligned}
& \delta \mathbf{D}=\left[\left(\frac{3 \cdot \mathbf{M}_{\mathbf{d}}\left(\alpha, \mathbf{E}_{\mathbf{f}}\right)}{4 \cdot \pi \cdot \rho_{\mathbf{D o}}}\right)+\left(\xi \cdot \mathbf{r}_{\mathbf{a g}}\right)^{3}\right]^{0.333}-\xi \cdot \mathbf{r}_{\mathrm{ag}} \quad \mathbf{r}_{\mathbf{a g}}=\left[\left(\frac{3 \cdot \mathbf{M}_{\mathbf{h}}\left(\alpha, \mathbf{E}_{\mathbf{f}}\right)}{4 \cdot \pi \cdot \rho_{\mathbf{H o}}}\right)+\left(\xi \cdot \mathbf{r}_{\mathbf{a g}}+\delta \mathbf{D}\right)^{3}\right]^{0.333} \\
& \operatorname{Sol}\left(\alpha, \mathbf{E}_{\mathbf{f}}\right):=\operatorname{Find}\left(\delta \mathbf{D}, \mathbf{r}_{\mathbf{a g}}\right) \\
& \delta_{\mathrm{LD}}\left(\alpha, \mathbf{E}_{\mathbf{f}}\right):=\operatorname{Sol}\left(\alpha, \mathbf{E}_{\mathbf{f}}\right) \mathbf{0} \\
& \mathbf{w a o l}_{\mathbf{m}}\left(\alpha, \mathbf{E}_{\mathbf{f}}\right):=\operatorname{Sol}\left(\alpha, \mathbf{E}_{\mathbf{f}}\right) \mathbf{1} \\
& \text { Initial D2 layer thickness } \\
& \text { Initial outer } \mathrm{H} 2 \text { ablator radius } \quad \mathrm{r}_{\mathrm{ao}}(1.5,1)=0.489 \\
& \text { ORIGIN }:=0 \quad \quad \mathrm{im}:=31 \quad \lambda_{n}:=\frac{0.5 \cdot \mathrm{jm}}{(0.5 \cdot \mathrm{jm}+1)^{3}} \quad \chi=0 \quad \delta \mathrm{jto}_{\mathrm{w}}:=130 \cdot 10^{-9} \quad \mathrm{~s} \\
& \underset{\mathbf{k}:=\mathbf{0} . . \mathbf{j m}-\mathbf{2}}{\mathbf{j}:=\mathbf{0} . \mathbf{j m}} \quad \delta \mathbf{t}_{\mathbf{j}}:=\left[\Phi\left[\frac{\mathbf{j}}{(\mathbf{j}+\mathbf{1})^{\mathbf{3}}}-\chi\right] \cdot \frac{\mathbf{j}}{(\mathbf{j}+\mathbf{1})^{\mathbf{3}}}+\Phi\left[\chi-\frac{\mathbf{j}}{(\mathbf{j}+\mathbf{1})^{\mathbf{3}}}\right] \cdot \chi\right] \cdot \delta \mathbf{t o}^{\mathbf{3}} \quad \mathbf{t}_{\mathbf{j}}:=\sum_{\mathbf{n}=\mathbf{0}}^{\mathbf{j}} \delta \mathbf{t}_{\mathbf{n}} \\
& \text { jm-1 }=30 \quad \mathrm{M}_{\mathrm{a}_{\mathrm{h}}}:=2 \cdot \mathrm{M}_{\mathbf{h}}(1.5,1) \quad \mathrm{M}_{\mathbf{0}}=\mathbf{0 . 0 4 6} \quad \mathrm{g} \quad \delta \mathrm{M}:=\frac{0.5}{\mathrm{jm}-1} \cdot \mathrm{M}_{\mathbf{0}} \\
& (\mathbf{j m}-\mathbf{1}) \cdot \delta \mathrm{M}=\mathbf{0 . 0 2 3} \quad \mathrm{M}_{\mathbf{0}}:=\mathrm{M}_{\mathbf{0}} \quad \mathbf{0 . 5} \cdot \mathbf{M}_{\mathbf{0}}=\mathbf{0 . 0 2 3} \quad \mathrm{M}_{\mathbf{j}}:=\mathrm{M}_{\mathbf{0}}-\mathbf{j} \cdot \delta \mathrm{M} \\
& M_{d}(1.5,1)+M_{t}(1.5,1)=0.023 \quad \delta M=7.6601 \times 10^{-4} \quad 0.5 \cdot M_{0} \cdot(j m-1)^{-1}=7.6601 \times 10^{-4}
\end{aligned}
$$

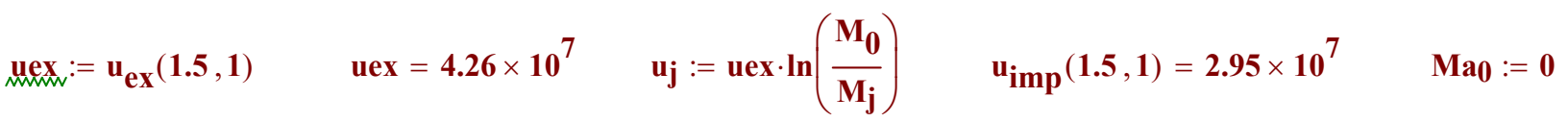

$$
\begin{aligned}
& M_{\mathbf{j}}:=M_{0}-M_{j} \quad \operatorname{Eex}_{\mathbf{j}}:=0.5 \cdot 10^{-13} \cdot \mathrm{Ma}_{\mathbf{j}} \cdot \mathbf{u e x}^{2}+10^{-5} \quad \mathrm{Ep}_{\mathrm{j}}:=0.5 \cdot 10^{-13} \cdot \mathrm{M}_{\mathrm{j}} \cdot\left(\mathrm{u}_{\mathrm{j}}\right)^{2}
\end{aligned}
$$

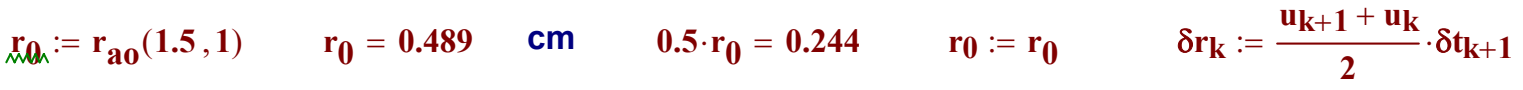

$$
\begin{aligned}
& r_{k}:=r_{0}-\sum_{n=0}^{k}\left(\delta r_{n}\right) \quad \operatorname{Pex}_{k}:=\frac{\left(\operatorname{Eex}_{k+1}-E x_{k}\right) \cdot 10^{6}}{\delta t_{k+1}} \quad \sum_{n=0}^{j m-2}\left(\operatorname{Pex}_{n} \cdot \delta t_{n+1}\right)=2.08 \times 10^{6} \\
& \text { uak }_{k}:=\text { uex }-u_{k} \quad r_{j m-1}:=0.22 \quad r a_{k}:=r_{k}+u a_{k} \cdot \sum_{n=k}^{j m-2} \delta t_{n+1} \quad \delta \rho a_{k}:=\delta M \cdot\left[4 \cdot \frac{\pi}{3}\left[\left(r_{k}\right)^{3}-\left(r_{k+1}\right)^{3}\right]\right]^{-1} \\
& \delta \rho r_{\mathbf{k}}:=\delta \rho \mathbf{a}_{\mathbf{k}} \cdot\left(\mathbf{r a}_{\mathbf{k}}-\mathbf{r}_{\mathbf{k}+1}\right) \quad \operatorname{\rho ra}_{\mathbf{k}}:=\sum_{\mathbf{n}=\mathbf{0}}^{\mathbf{k}} \delta \rho \mathrm{a}_{\mathbf{n}} \quad \operatorname{\rho rh}_{\mathbf{k}}:=\frac{\mathbf{0 . 5} \cdot \mathbf{M}_{\mathbf{0}}-\mathbf{k} \cdot \delta \mathbf{M}}{4 \cdot \pi \cdot\left(\mathbf{r}_{\mathbf{k}}\right)^{2}} \quad \mathbf{g} / \mathrm{cm}^{2}
\end{aligned}
$$

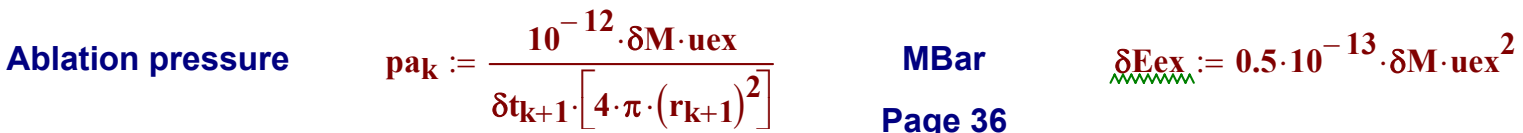


Table 9. Parameters during implosion for the $\mathrm{Mo} / \mathrm{Mf}=2$ case $\mathrm{C}$

$\operatorname{Pex}_{30}:=155 \cdot 10^{12} \quad \mathrm{r}_{30}:=0.21 \quad \operatorname{ra30}_{30}:=0.22 \quad \operatorname{pra}_{30}:=12 \cdot 10^{-3} \quad \operatorname{prh}_{30}:=0 \quad \operatorname{pa}_{30}:=130$

\begin{tabular}{|c|c|c|c|c|c|c|c|c|c|c|c|}
\hline$\frac{t_{m}}{10^{-9}}$ & $\frac{M_{m}}{10^{-3}}$ & $\frac{u_{m}}{10^{7}}$ & $\operatorname{Eex}_{\mathbf{m}}=$ & $\mathbf{E} \mathbf{p}_{\mathbf{m}}=$ & $\frac{\mathbf{E} \mathbf{p}_{m}}{E \mathbf{E x}_{m}}$ & $\frac{P e x_{m}}{10^{12}}$ & $\mathbf{r}_{\mathbf{m}}=$ & $\mathrm{ra}_{\mathbf{m}}=$ & $\frac{\rho \mathrm{pa}_{\mathrm{m}}}{10^{-3}}$ & $\frac{\rho \mathrm{rh}_{\mathrm{m}}}{10^{-3}}$ & $\mathbf{p a}_{\mathbf{m}}=$ \\
\hline 0.4 & 46 & 0 & 0 & 0 & 0 & 4 & 0.48 & 2.89 & 0.02 & 7.8 & 0.7 \\
\hline 26.3 & 44 & 0.14 & 0.14 & 0.005 & 0.03 & 11 & 0.46 & 1.73 & \begin{tabular}{l|l|}
0.1 \\
\end{tabular} & 8 & 2.1 \\
\hline 36.6 & 43 & 0.29 & 0.28 & 0.018 & $\begin{array}{l}0.07 \\
\end{array}$ & 23 & 0.44 & 1.25 & 0.24 & 8.2 & 4.6 \\
\hline $\begin{array}{l}41.9 \\
\end{array}$ & 41 & 0.45 & 0.42 & 0.042 & 0.1 & 39 & 0.42 & 1 & 0.45 & 8.2 & 8.5 \\
\hline \begin{tabular}{|l|}
45.1 \\
\end{tabular} & 40 & 0.61 & 0.56 & 0.074 & \begin{tabular}{|l|}
0.13 \\
\end{tabular} & 59 & 0.41 & 0.85 & 0.73 & \begin{tabular}{|l|}
8.1 \\
\end{tabular} & 13.9 \\
\hline $\begin{array}{l}47.2 \\
\end{array}$ & 38 & 0.78 & 0.69 & 0.115 & 0.17 & 84 & 0.39 & 0.74 & 1.09 & 7.9 & 20.9 \\
\hline \begin{tabular}{ll|}
48.8 \\
\end{tabular} & 37 & 0.95 & $\begin{array}{ll}0.83 \\
\end{array}$ & 0.166 & 0.2 & 113 & 0.38 & 0.66 & 1.52 & 7.6 & 30 \\
\hline 499.9 & 35 & 1.13 & 0.97 & 0.225 & 0.23 & 146 & 0.37 & 0.59 & 2.02 & 7.2 & 41.2 \\
\hline 50.8 & 34 & 1.32 & 1.11 & 0.294 & 0.26 & 155 & 0.36 & 0.54 & 2.6 & 6.7 & 47 \\
\hline 51.7 & 32 & 1.52 & 1.25 & 0.371 & 0.3 & 155 & 0.34 & 0.49 & 3.28 & 6.2 & 51.1 \\
\hline 52.6 & 31 & 1.73 & 1.39 & 0.456 & 0.33 & 155 & 0.33 & 0.44 & 4.08 & 5.7 & 56.5 \\
\hline 53.5 & 29 & 1.94 & 1.53 & 0.55 & 0.36 & 155 & 0.31 & 0.39 & 5.03 & 5 & 63.7 \\
\hline 54.4 & 28 & 2.17 & 1.67 & 0.652 & 0.39 & 155 & 0.29 & 0.35 & 6.2 & \begin{tabular}{ll|}
4.3 \\
\end{tabular} & 73.3 \\
\hline 55.3 & 26 & 2.42 & 1.8 & 0.761 & 0.42 & 155 & 0.27 & $\begin{array}{ll}0.3 \\
\end{array}$ & 7.66 & \begin{tabular}{|l|}
3.4 \\
\end{tabular} & 86.9 \\
\hline 56.2 & 25 & 2.68 & 1.94 & \begin{tabular}{|c|}
0.877 \\
\end{tabular} & 0.45 & 155 & 0.25 & 0.26 & 9.55 & 2 & \begin{tabular}{|l|}
106.8 \\
\end{tabular} \\
\hline 57.1 & 23 & 2.95 & 2.08 & 1 & 0.48 & 155 & 0.21 & 0.22 & 12 & 0 & 130 \\
\hline
\end{tabular}

Fig. 28: Case C $E_{f}=1 M J$ implosion dynamics for lower mass ablator case $M_{0} / M_{f}=2$ (more laser-like rocket)

Ablation front radius $\mathrm{r}(\mathrm{cm})$

Exhaust energy $E_{\text {ex }}(M J)$ Ablation pressure $p_{a}$ (in100 Mbar units)

Rocket K.E. power $P_{e x}$ (in 100 TW units). (required beam power is roughly $3 X$ higher)
Time axis in ns

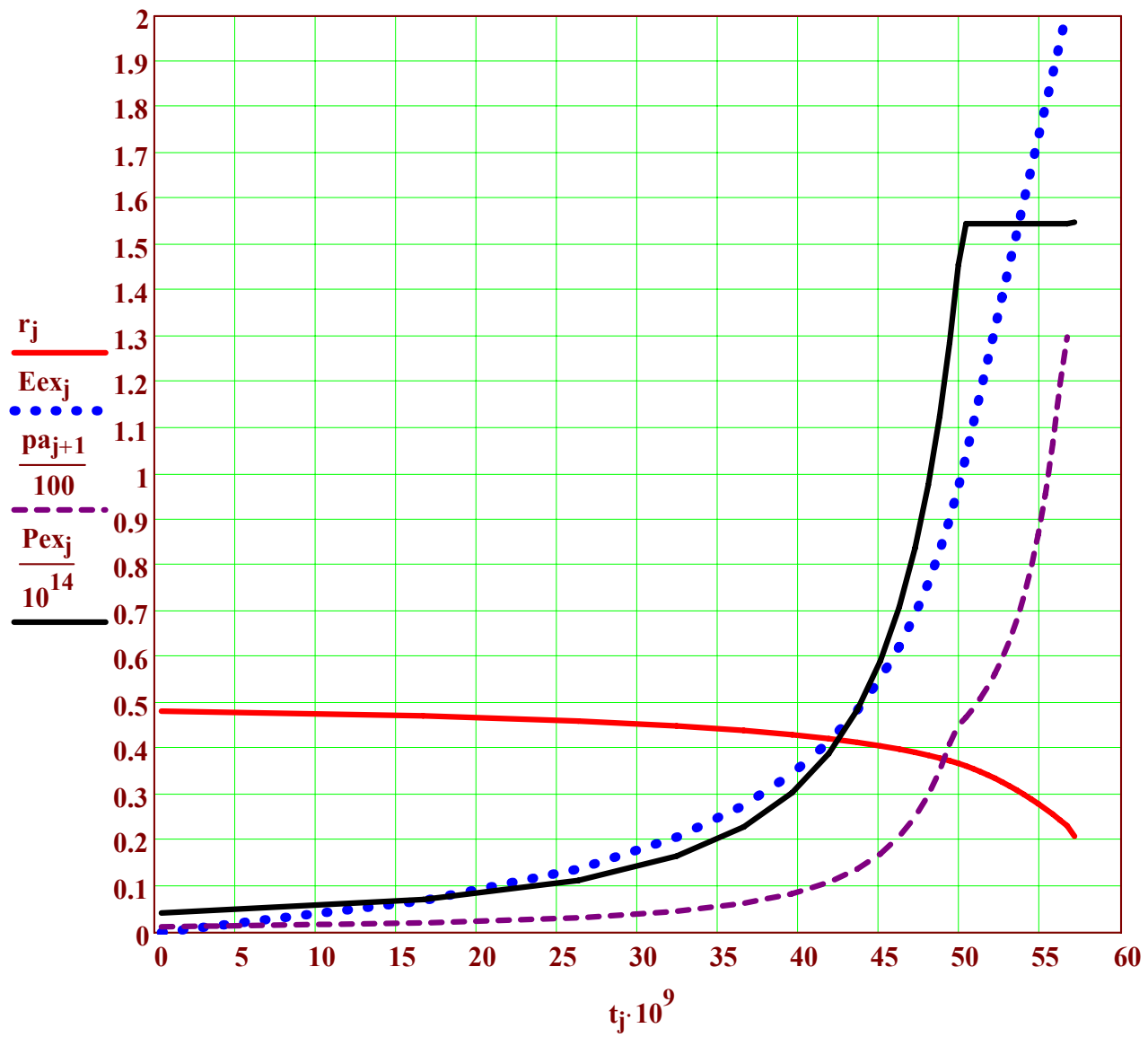

Page 37 
Given beam range penetrating ablator

Required beam range versus $t(k)$ for case C $\delta_{\text {dor }}:=\mathbf{0 . 0 0 2}$ $\mathrm{g} / \mathrm{cm}^{2}$ (assumed constant) must be small fraction of total initial ablator $\rho r_{\text {ho }}$

Fig. 29: Case B Column densities ( $\rho r)$ 's:

(g/cm² units)

Beam range in ablator $\delta \rho r_{b h}$ Ablated plasma $\rho r_{a}$ (dotted)

Total req. beam range incident

Remaining ablator $\rho r_{h}$

$\rho \mathbf{r}_{\mathbf{b}}\left(\rho \mathbf{r}_{\mathbf{a}}\right):=\rho \mathbf{r}_{\mathbf{a}}+\delta \rho \mathbf{r}_{\mathbf{b h}} \quad \rho \mathbf{r b}_{\mathbf{k}}:=\rho \mathbf{r}_{\mathbf{b}}\left(\rho \mathbf{r a}_{\mathbf{k}}\right) \quad \mathbf{g} / \mathbf{c m}^{2}$

Time in ns

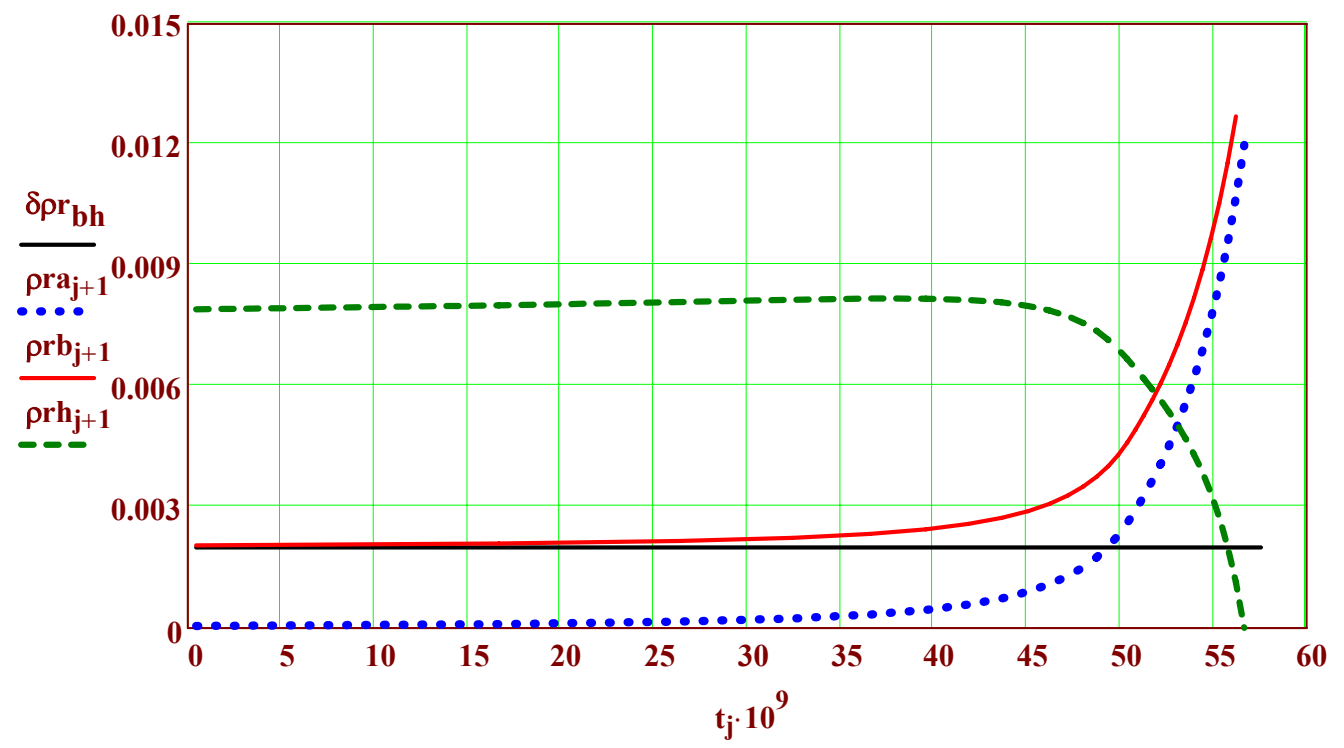

Calculation of ion beam requirements for Case C Power Plant $\mathrm{M}_{0} / \mathrm{M}_{\mathrm{f}}=2$ (Fig. 28)

$$
\begin{aligned}
& \delta M=7.66 \times 10^{-4} \quad \rho_{\mathrm{a}}=0.5 \quad \mathrm{~A}_{\text {if }}=15 \quad \text { uex }=4.26 \times 10^{7} \quad \mathrm{~A}_{\mathrm{b}}=40 \\
& \underset{\max }{\operatorname{Ta}}\left(\alpha, \mathrm{E}_{\mathrm{f}}\right):=3 \cdot 10^{-4} \cdot \mathrm{m}_{\mathrm{h}} \cdot \mathrm{u}_{\mathrm{ex}}\left(\alpha, \mathrm{E}_{\mathrm{f}}\right)^{2} \cdot 200^{-2} \cdot\left(1.6 \cdot 10^{-19}\right)^{-1} \quad \mathrm{~T}_{\mathrm{ex}}(1.5,1)=142 \quad \mathrm{eV} \\
& \underset{\mathrm{E}}{\mathbf{E}}\left(\alpha, \mathbf{E}_{\mathbf{f}}\right):=0.5 \cdot 10^{-13} \cdot \mathbf{M}_{\mathbf{h}}\left(\alpha, \mathbf{E}_{\mathbf{f}}\right) \cdot \mathbf{u}_{\mathrm{ex}}\left(\alpha, \mathbf{E}_{\mathbf{f}}\right)^{2} \ldots \\
& +\left(13.6 \cdot 2+3 \cdot T_{e x}\left(\alpha, E_{f}\right)\right) \cdot 1.6 \cdot 10^{-25} \cdot M_{h}\left(\alpha, E_{f}\right) \cdot m_{h}{ }^{-1} \ldots \\
& +\mathbf{E}_{\mathbf{c s}}\left(\alpha, \mathbf{E}_{\mathbf{f}}\right) \\
& \max \left(\alpha, \mathbf{E}_{\mathbf{f}}\right):=0.5 \cdot 10^{-13} \cdot \mathbf{M}_{\mathbf{h}}\left(\alpha, \mathbf{E}_{\mathbf{f}}\right) \cdot \mathbf{u}_{\mathrm{ex}}\left(\alpha, \mathbf{E}_{\mathbf{f}}\right)^{2} \cdot \mathbf{E}_{\mathbf{d a}}\left(\alpha, \mathbf{E}_{\mathbf{f}}\right)^{-1}
\end{aligned}
$$

Fraction of ablated $\rho$ in total beam $\rho r_{b} \quad f_{b} r_{k}:=\frac{\rho r_{k}}{\rho r_{b}\left(\rho r_{k}\right)} \quad Z_{b}=18$

Fraction of beam energy in ablated plasma $\mathbf{f b a}_{\mathbf{k}}:=\mathbf{f}_{\mathbf{b a}}\left(\rho_{\mathbf{a}}, \rho \operatorname{\rho rb}_{\mathbf{k}}, \mathrm{f}_{\mathrm{k}}, \mathbf{T}_{\mathbf{e}}, \mathbf{A}_{\mathbf{b}}, \mathbf{A}_{\mathbf{t}}, \mathbf{Z}_{\mathbf{b}}, \mathbf{Z}_{\mathbf{t}}\right)$

Required incident beam energy integrated up to time $t_{k}$

$$
\text { Ebinc }_{\mathbf{k}}:=\sum_{\mathbf{n}=\mathbf{0}}^{\mathbf{k}} \frac{\delta \text { Eex }}{\left(1-\mathrm{fba}_{\mathbf{n}}\right) \cdot \eta_{\mathrm{ex}}(1.5,1)}
$$

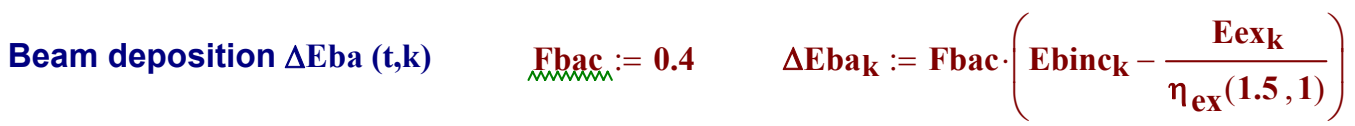

MJ

\section{Page 38}




$$
\operatorname{Mba}_{k}:=2 \cdot \rho \operatorname{ra}_{k} \cdot \pi \cdot\left(\mathbf{r}_{\mathrm{k}+1}\right)^{2}
$$

Now lets reduce all $\rho r a ' s$ by the factor far:

Fraction of ablated $\rho r$ in total beam $\rho r_{b}$

Fraction of beam energy in ablated plasma $\operatorname{far}_{\mathbf{k}}:=\left(\mathbf{r}_{\mathbf{k}}\right)^{2} \cdot\left[\mathbf{r}_{\mathbf{k}}+\mathbf{v}_{\operatorname{aperp}}\left(\Delta \mathbf{E b a}_{\mathbf{k}}, M_{\mathrm{Mba}}\right) \cdot\left(\sum_{\mathbf{n}=\mathbf{k}}^{\mathrm{jm}-1} \delta \mathbf{t}_{\mathbf{n}+1}\right)\right]^{-2}$ $\rho \operatorname{rb}_{\mathbf{k}}:=\rho \mathbf{r}_{\mathbf{b}}\left(\rho \mathbf{r a}_{\mathbf{k}} \cdot \mathbf{f a r}_{\mathbf{k}}\right) \quad \mathbf{g} / \mathbf{c m}^{2}$

$\operatorname{forc}_{\mathbf{k}}:=\frac{\rho \mathbf{r a}_{\mathbf{k}} \cdot \mathbf{f a r}_{\mathbf{k}}}{\rho \mathbf{r}_{\mathbf{b}}\left(\rho \mathbf{r a}_{\mathbf{k}} \cdot \mathbf{f a r}_{\mathbf{k}}\right)}$

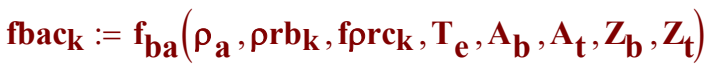

$$
\begin{aligned}
& \operatorname{far}_{\mathbf{j}+1} \\
& \frac{\text { fba }_{\mathbf{j}+1}}{\text { fbac }_{\mathbf{j}+1}} \\
& \frac{\mathbf{v a p e r p}_{\text {aex }}\left(\Delta \mathbf{E b a}_{\mathbf{j}+1}, \mathbf{M b a}_{\mathbf{j}+1}\right)}{u \text { uex }}
\end{aligned}
$$

time in ns

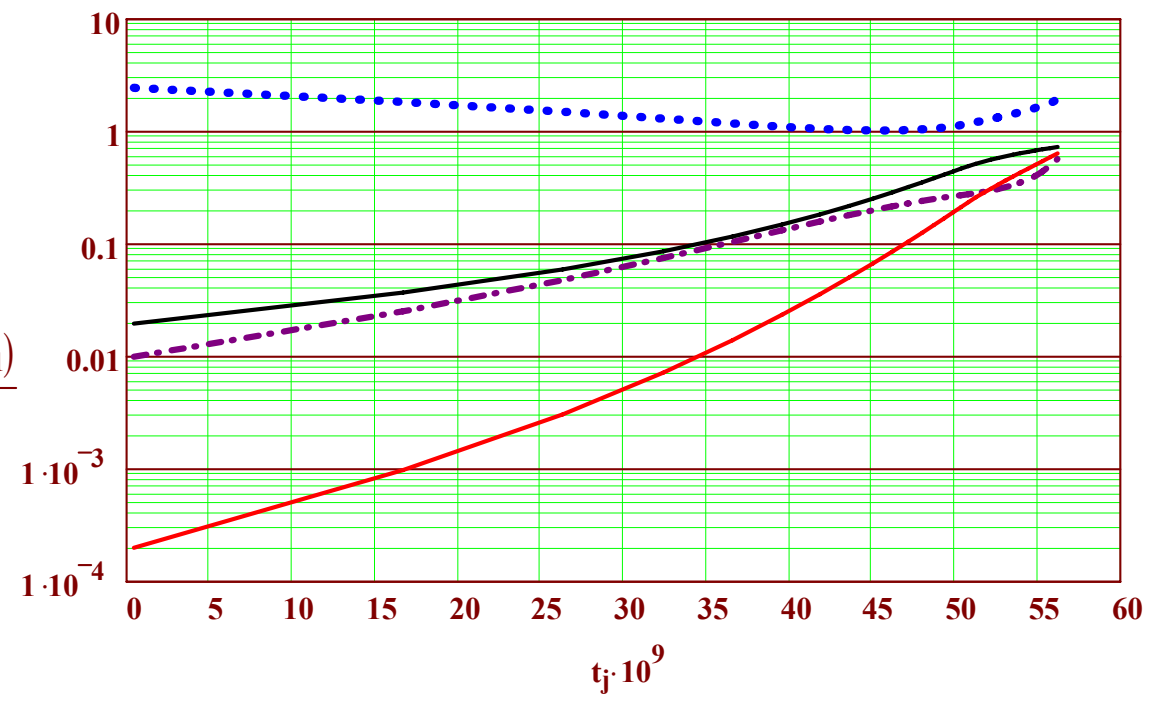

Figure 30: Ablated plasma expansion factors (column density $\rho r_{a}$ reduction factors) $f_{\text {ar }}$, and beam loss fractions without corrections for expansion $f_{b a}$ (For case $C$ as in Fig 28) versus time during implosion and with corrections for expansion $f_{b a c}$ (red curve). Note that taking into account ablated plasma heating with expansion in the expected 2-D beam geometry reduces net beam attenuation significantly. The coefficient Fbac $=0.4$ used in the self consistent beam energy deposition was obtained from the value of fbac at the time of 53 ns when the remaining ablator $\rho$ rh equaled the ablated plasma $\rho$ ra in Fig. 29. Actual 2-D hydro implosion calculations will be needed to get more accurate estimates of beam loss in 2-D. Note the net beam heating is sufficient to give perpendicular plasma expansion velocities comparable to the radial uex.

Incident beam ion K.E.

Required incident beam energy integrated up to time $t_{k}$

$$
\mathbf{E b o}_{\mathbf{k}}:=\mathbf{E}_{\mathbf{b f}}\left(\rho_{\mathbf{a}}, \rho \operatorname{prb}_{\mathbf{k}}, \mathbf{0 . 0 1}, \mathbf{T}_{\mathbf{e}}, \mathbf{A}_{\mathbf{b}}, \mathbf{A}_{\mathbf{t}}, \mathbf{Z}_{\mathbf{b}}, \mathbf{Z}_{\mathbf{t}}\right) \quad \mathbf{M e V}
$$$$
\text { Ebinc }_{\mathbf{k}}:=\sum_{\mathbf{n}=\mathbf{0}}^{\mathbf{k}} \frac{\delta \mathrm{Eex}}{\left(1-\mathrm{fbac}_{\mathbf{n}}\right) \cdot \eta_{\mathrm{ex}}(1.5,1)}
$$

Ebinc $_{j m-2}=4.39<--23 \%$ beam to fuel coupling efficiency- $2 \%$ less than for $M_{o} / M_{f}=5$ Case A

Required incident beam power versus $k$

Incident beam intensity at radius $r_{k}$

$$
\text { Pbinc }_{k}:=\frac{10^{-6} \cdot \delta \text { Eex }}{\delta t_{k+1} \cdot\left(1-\text { fback }_{k}\right) \cdot \eta_{e x}(1.5,1)}
$$$$
\text { Ibinck }_{k}:=\operatorname{Pbinc}_{\mathrm{k}} \cdot\left[4 \cdot \pi \cdot\left(\mathrm{r}_{\mathrm{k}}\right)^{2}\right]^{-1}
$$

TW

$\mathrm{TW} / \mathrm{cm}^{2}$

Page 39 


$$
A_{b}=40 \quad Z_{t}=1
$$

Argon beams

Incident beam energy $(\mathrm{GeV})$

Fractional beam loss in ablated plasma at local time $t_{k}$ Incident beam power (PW) Incident beam intensity $\left(\mathrm{PW} / \mathrm{cm}^{2}\right)$

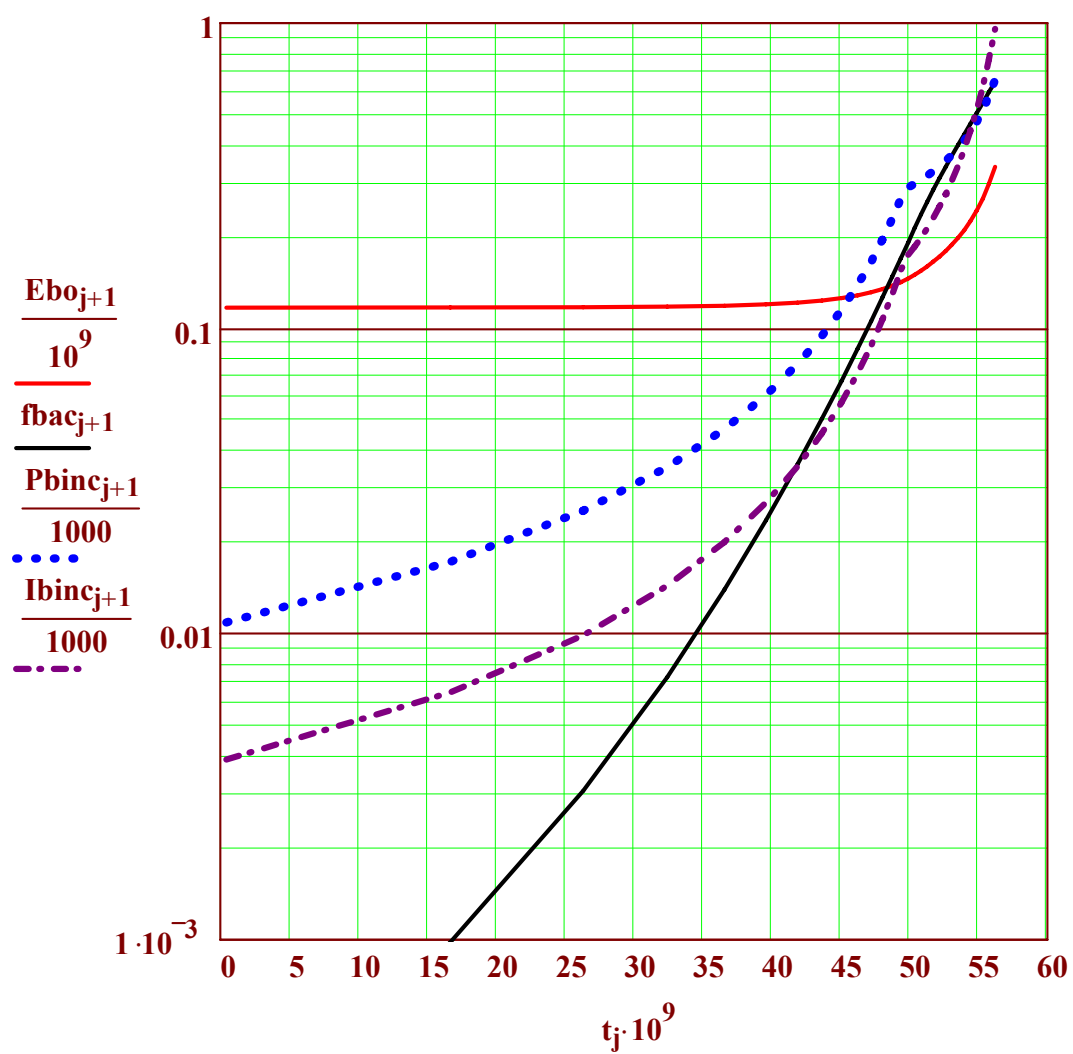

Time axis in ns-->

$$
\mathrm{t}_{\mathbf{j}} \cdot 10^{9}
$$

Figure 31. Beam requirements for the Case $C-M_{0} / M_{f}=2$ laser-like ablation regime. This case gives $2 \%$ less coupling efficiency than Case $A-M_{o} / M_{f}=5$, and has considerably more difficult beam requirements ( $\sim$ three times the peak beam power and intensity, $40 \%$ smaller focal spot radius, and at half the beam ion kinetic energy --much much higher combined difficulty for the modular induction driver to deliver. In addition, the ablator energy density and ablation front stagnation temperature $\sim 100 \mathrm{eV}$ is no longer optically thick to bremstrahlung radiation, so that preheat cannot be neglected in this case as it can be in Case A, or one must a small amount of carbon (plastic) to increase opacity.

Lets now change back to the $M_{o} / M_{f}=5$ case with higher rocket efficiency for the 2-D calculations.

$$
\begin{aligned}
& \mathbf{M}_{\mathbf{m}}\left(\alpha, \mathbf{E}_{\mathbf{f}}\right):=4 \cdot\left(\mathbf{M}_{\mathbf{d}}\left(\alpha, \mathbf{E}_{\mathbf{f}}\right)+\mathbf{M}_{\mathbf{t}}\left(\alpha, \mathbf{E}_{\mathbf{f}}\right)\right) \\
& M_{h}(1.5,1)=0.092 \\
& \underset{\operatorname{unx}}{u_{(}}\left(\alpha, \mathbf{E}_{\mathbf{f}}\right):=\mathbf{u}_{\mathrm{imp}}\left(\alpha, \mathbf{E}_{\mathbf{f}}\right) \cdot \ln (\mathbf{5})^{-1} \\
& \mathrm{u}_{\mathrm{ex}}(1.5,1)=1.83 \times 10^{7} \\
& \underset{\max }{\operatorname{Tox}}\left(\alpha, \mathrm{E}_{\mathrm{f}}\right):=3 \cdot 10^{-4} \cdot \mathrm{m}_{\mathrm{h}} \cdot \mathrm{u}_{\mathrm{ex}}\left(\alpha, \mathrm{E}_{\mathrm{f}}\right)^{2} \cdot 200^{-2} \cdot\left(1.6 \cdot 10^{-19}\right)^{-1} \\
& T_{\text {ex }}(1.5,1)=26 \\
& \mathbf{E}_{\mathbf{d a}}(1.5,1)=2.55 \\
& \underset{\mathrm{E} d a x}{\mathbf{E}_{\mathrm{f}}}\left(\alpha, \mathbf{E}_{\mathbf{f}}\right):=0.5 \cdot 10^{-13} \cdot \mathrm{M}_{\mathbf{h}}\left(\alpha, \mathbf{E}_{\mathbf{f}}\right) \cdot \mathbf{u}_{\mathrm{ex}}\left(\alpha, \mathbf{E}_{\mathbf{f}}\right)^{2} \ldots \\
& +\left(13.6 \cdot 2+3 \cdot T_{\text {ex }}\left(\alpha, E_{f}\right)\right) \cdot 1.6 \cdot 10^{-25} \cdot M_{h}\left(\alpha, E_{f}\right) \cdot m_{h}{ }^{-1} \ldots \\
& +\mathbf{E}_{\mathbf{c s}}\left(\alpha, \mathbf{E}_{\mathbf{f}}\right) \\
& \max \left(\alpha, \mathbf{E}_{\mathbf{f}}\right):=0.5 \cdot 10^{-13} \cdot \mathbf{M}_{\mathbf{h}}\left(\alpha, \mathbf{E}_{\mathbf{f}}\right) \cdot \mathbf{u}_{\mathrm{ex}}\left(\alpha, \mathbf{E}_{\mathbf{f}}\right)^{2} \cdot \mathbf{E}_{\mathrm{da}}\left(\alpha, \mathbf{E}_{\mathbf{f}}\right)^{-1} \\
& \eta_{\mathrm{ex}}(1.5,1)=0.61
\end{aligned}
$$

\section{Page 40}




\section{Rough power plant parameters and cost model}

We will consider both cases with CHFAR MHD Balance of Plant only, and cases with an added steam bottoming cycle. in the latter case the steam bottoming cycle will add $35 \%$ of the unconverted plasma power exiting the MHD generator, but the cost per kilowatt for the steam cycle is much higher, and the optimum choice we will see depends on the target gain, which is the biggest factor that determines the relative importance of the driver cost vs the balance of plant cost. We will adopt the cost scalings for both cases given in reference 5 , more or less reproduced below. To account for the scale lengths of chamber size with yield, we assign somewhat arbitrarily the following pulse repetition rates $R R$ as a function of fuel energy $E_{f}$ :

$8 \mathrm{~Hz}$ for $0.2 \mathrm{MJ}, 6 \mathrm{~Hz}$ for $1 \mathrm{MJ}$, and $5 \mathrm{~Hz}$ for $2.6 \mathrm{MJ}$.

[5] B. G. Logan, Lawrence Livermore National Laboratory Report UCRL-ID-110129 (July 1992)

Gross electric output per pulse

$$
\mathbf{W}_{\mathbf{e}}\left(\eta_{\operatorname{con}}, \alpha, \mathbf{E}_{\mathbf{f}}\right):=\eta_{\text {con }} \cdot\left(1-\mathbf{F Y}_{\mathbf{n b}}\left(\alpha, \mathbf{E}_{\mathbf{f}}\right)\right) \cdot \mathbf{Y}_{\mathbf{f}}\left(\alpha, \mathbf{E}_{\mathbf{f}}\right) \quad \mathbf{M J}_{\mathbf{e}} / \text { pulse }
$$

Net electric output per pulse

$$
\mathbf{W}_{\text {net }}\left(\eta_{\text {con }}, \eta_{\mathbf{d f}}, \eta_{\mathbf{d}}, \alpha, \mathbf{E}_{\mathbf{f}}\right):=\eta_{\mathbf{c o n}} \cdot \mathbf{0 . 9 5} \cdot\left(1-\mathbf{F Y} \mathbf{Y}_{\mathbf{n b}}\left(\alpha, \mathbf{E}_{\mathbf{f}}\right)\right) \cdot \mathbf{Y}_{\mathbf{f}}\left(\alpha, \mathbf{E}_{\mathbf{f}}\right)-\mathbf{E}_{\mathbf{f}} \cdot \eta_{\mathbf{d f}}{ }^{-1} \cdot \eta_{\mathbf{d}}{ }^{-1} \quad \mathbf{M J}_{\mathbf{e}} / \text { pulse }_{\text {Eq } 83}
$$

We will provide most detail for two cases $A$ (the reference CFAR Power Plant with $E_{f}=1 \mathrm{MJ}$ fuel energy and case $B$ ( a CFAR demo with $E_{f}=0.2 \mathrm{MJ}$, the net $T$-breeding breakeven point), and just a bit at the end for a very large plant with $E_{f}=2.6 \mathrm{MJ}$ fuel energy (ultimate low CoE). For the demo case we will find that the lowest $\mathrm{CoE}$ occurs with a steam bottoming plant included, as so for DEMO we use an estimated $\eta_{\text {con }}=\left(\eta_{\text {MHD }}=0.4\right)+\left(\eta_{\text {steam }}=0.35^{*}\left(1-\eta_{M H D}\right)\right)=0.65$, the same as the efficiency with MHD conversion alone at the larger yield power plant case A (see Fig. 3 efficiency plot). Powers in $M W$, costs in $M \$$ of direct costs, to be multiplied by 2.8 , indirect cost + assembly multiplier in CoE.

$$
W_{\text {netA }}:=W_{\text {net }}\left(0.65, \eta_{\text {dfA }}, 0.4,1.5,1\right) \quad W_{\text {netB }}:=W_{\text {net }}\left(0.65, \eta_{\text {dfB }}, 0.2,1.5,0.2\right) \quad \mathrm{MJ}_{\mathrm{e}} / \text { pulse }
$$

Net electric power

$$
\begin{aligned}
& \mathbf{P}_{\text {net }}\left(\mathbf{R R}, \eta_{\mathbf{c o n}}, \eta_{\mathbf{d f}}, \eta_{\mathbf{d}}, \alpha, \mathbf{E}_{\mathbf{f}}\right):=\mathbf{R R} \cdot \mathbf{w}_{\text {net }}\left(\eta_{\text {con }}, \eta_{\mathbf{d f}}, \eta_{\mathbf{d}}, \alpha, \mathbf{E}_{\mathbf{f}}\right) \\
& \text { MWe } \\
& P_{\text {netA }}:=P_{\text {net }}\left(6,0.65, \eta_{\text {dfA }}, 0.4,1.5,1\right) \quad P_{\text {netB }}:=P_{\text {net }}\left(8,0.65, \eta_{\text {dfB }}, 0.2,1.5,0.2\right)
\end{aligned}
$$

Modular solenoid driver (+driver bldg cost). Here we use last years study, ref

[6] B. G. Logan, "Small Modular Driver Study" Lawrence Berkeley National Lab Report May 2006. See web site www.osti.gov/servlets/purl/902800-eyJKrw/ ) We take the driver cost to be linear with driver energy because of high modularity.

\$/J beam direct cost.

$$
\begin{aligned}
& \mathbf{C}_{\text {driver }}\left(\eta_{\mathbf{d f}}, \mathbf{E}_{\mathbf{f}}\right):=\mathbf{U D C}_{\text {driver }} \cdot \frac{\mathbf{E}_{\mathbf{f}}}{\eta_{\mathbf{d f}}} \\
& C_{\text {driver }}\left(\eta_{\text {dfA }}, 1\right)=527 \quad C_{\text {driver }}\left(\eta_{\text {dfB }}, 0.2\right)=111 \quad M \$ \text { direct cost }
\end{aligned}
$$

$$
\text { UDC }_{\text {driver }}:=130
$$

Eq 85

Cost of reactor vessel inc vessel magnet (twice the HYLIFE-II vessel cost because of the magnets)

$$
\begin{gathered}
\mathrm{C}_{\text {vessel }}\left(\alpha, \mathrm{E}_{\mathbf{f}}\right):=60 \cdot\left(\frac{\mathrm{Y}_{\mathbf{f}}\left(\alpha, \mathrm{E}_{\mathbf{f}}\right)}{500}\right)^{0.8} \quad \begin{array}{l}
\mathrm{M} \$ \quad \begin{array}{l}
\text { (assumes magnet cost scales } \\
\text { with magnetic stored energy } \sim \text { plasma yield) }
\end{array} \\
\mathrm{C}_{\text {vessel }}(1.5,1)=59 \quad \mathrm{C}_{\text {vessel }}(1.5,0.2)=8 \quad \mathrm{M} \$, \text { direct cost }
\end{array}
\end{gathered}
$$


Balance of Plant costs (MHD only and MHD+steam bottoming)

$$
\begin{aligned}
& \mathrm{C}_{\text {mhdBoP }}(\text { Pnet }):=0.07 \cdot \text { Pnet } \cdot\left(\frac{1400}{\text { Pnet }}\right)^{0.27} \quad C_{\text {steamBoP }}(\text { Pnet }):=0.57 \cdot \frac{\text { Pnet }}{2} \cdot\left(\frac{1400}{\text { 0.5.Pnet }}\right)^{0.1} \quad \begin{array}{l}
\text { M\$ } \quad \text { Eq } 87 \\
\text { direct }
\end{array} \\
& \mathrm{C}_{\text {mhdBoP }}(1500)=103 \quad \mathrm{C}_{\text {steamBoP }}(1500)=455 \quad M \$ \text {, direct } \\
& \mathrm{C}_{\text {mhdBoP }}(120)=16 \quad \mathrm{C}_{\text {steamBoP }}(120)=47 \quad \mathrm{M} \$ \text { direct }
\end{aligned}
$$

Other costs: aux pumps security bldgs, maintenance equip, rad waste

$$
\begin{aligned}
& C_{\text {other }}(\text { Pnet }):=100 \cdot\left(\frac{\text { Pnet }}{1400}\right)^{0.4} \quad \text { M\$ direct } \\
& C_{\text {other }}(1500)=103 \quad C_{\text {other }}(120)=37
\end{aligned}
$$

Eq 88

Operating costs-here we treat operation fuel costs $\mathrm{OT}_{\text {fuel }}$ (target and target-shell factory on site with recycled materials in the Rankine cycle, and regular O\&M costs

$$
\begin{aligned}
& \text { Cost per target+target shell } \quad C_{\text {target }}\left(\mathrm{RR}, \alpha, \mathrm{E}_{\mathrm{f}}\right):=\left[0.15+6 \cdot\left(0.002 \cdot \frac{\mathrm{Y}_{\mathrm{f}}\left(\alpha, \mathrm{E}_{\mathrm{f}}\right) \cdot 3.7}{500}\right)^{0.8}\right] \cdot\left(\frac{6}{\mathrm{RR}}\right)^{0.4} \quad \$ / \text { target } \\
& \mathrm{C}_{\text {target }}(6,1.5,1)=0.27 \quad \mathrm{C}_{\text {target }}(8,1.5,0.2)=0.15
\end{aligned}
$$

Operating charge for fueling (target and target-shell factory)

$$
\begin{aligned}
& \mathrm{OT}_{\text {fuel }}\left(\mathbf{R R}, \eta_{\text {con }}, \eta_{\mathbf{d f}}, \eta_{\mathbf{d}}, \alpha, \mathbf{E}_{\mathbf{f}}\right):=\mathbf{C}_{\text {target }}\left(\mathbf{R R}, \alpha, \mathbf{E}_{\mathbf{f}}\right) \cdot \frac{3600 \cdot \mathbf{R R}}{\mathbf{P}_{\text {net }}\left(\mathbf{R R}, \eta_{\operatorname{con}}, \eta_{\mathbf{d f}}, \eta_{\mathbf{d}}, \alpha, \mathbf{E}_{\mathbf{f}}\right)} \quad \mathrm{mills} / \mathrm{kW}_{\mathrm{e}} \mathrm{hr} \\
& \mathrm{OT}_{\text {fuel }}\left(6,0.65, \eta_{\mathrm{dfA}}, 0.4,1.5,1\right)=3.8 \quad \mathrm{OT}_{\text {fuel }}\left(8,0.65, \eta_{\mathrm{dfB}}, 0.2,1.5,0.2\right)=35 \quad \mathrm{mills} / \mathrm{kW}_{\mathrm{e}} \mathrm{hr} \\
& \operatorname{OM}\left(R R, \eta_{\text {con }}, \eta_{\mathbf{d f}}, \eta_{\mathbf{d}}, \alpha, \mathrm{E}_{\mathbf{f}}\right):=8 \cdot\left(\frac{1200}{P_{\text {net }}\left(\mathrm{RR}, \eta_{\text {con }}, \eta_{\mathbf{d f}}, \eta_{\mathbf{d}}, \alpha, \mathrm{E}_{\mathbf{f}}\right)}\right)^{0.5} \quad \mathrm{mills} / \mathrm{kW} \mathrm{e} \mathrm{hr} \quad \text { Eq } 91
\end{aligned}
$$

$\begin{array}{lllll}\text { Cost of Electricity: Fixed charge rate } & \text { FCR }:=0.1 \quad \text { Indirect cost } & \text { IND }:=2.8 \quad \text { Capacity factor } & \text { CF }:=0.9\end{array}$

$$
\begin{aligned}
& \left(\begin{array}{l}
\mathbf{C}_{\text {driver }}\left(\eta_{\mathbf{d f}}, \mathbf{E}_{\mathbf{f}}\right)+\mathbf{C}_{\text {vessel }}\left(\alpha, \mathbf{E}_{\mathbf{f}}\right) \ldots \\
+\mathbf{C}_{\mathbf{m h d B o P}}\left(\mathbf{P}_{\mathbf{n e t}}\left(\mathbf{R R}, \eta_{\mathbf{c o n}}, \eta_{\mathbf{d f}}, \eta_{\mathbf{d}}, \alpha, \mathbf{E}_{\mathbf{f}}\right)\right)
\end{array}\right) \cdots
\end{aligned}
$$

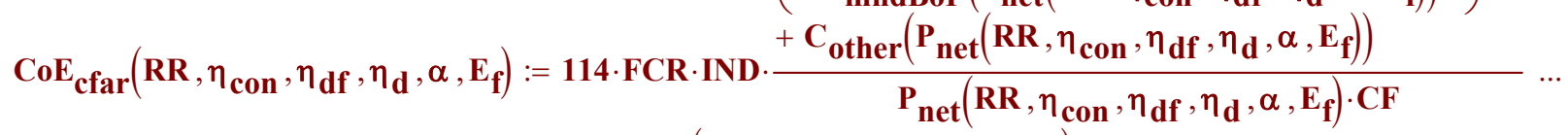

$$
\begin{aligned}
& +\operatorname{OT}_{\text {fuel }}\left(R R, \eta_{\text {con }}, \eta_{\mathbf{d f}}, \eta_{\mathbf{d}}, \alpha, \mathbf{E}_{\mathbf{f}}\right) \ldots \\
& +\operatorname{OM}\left(R R, \eta_{\text {con }}, \eta_{\mathbf{d f}}, \eta_{\mathbf{d}}, \alpha, \mathbf{E}_{\mathbf{f}}\right) \\
& \text { mills/kW } \mathrm{e}^{\mathrm{hr}} \quad \text { Eq } 92 \\
& \left(\begin{array}{l}
\mathbf{C}_{\text {driver }}\left(\eta_{\mathbf{d f}}, \mathbf{E}_{\mathbf{f}}\right)+\mathbf{C}_{\text {vessel }}\left(\alpha, \mathbf{E}_{\mathbf{f}}\right) \ldots \\
+\mathbf{C}_{\mathbf{m h d B o P}}\left(\mathbf{P}_{\mathbf{n e t}}\left(\mathbf{R R}, \eta_{\mathbf{c o n}}, \eta_{\mathbf{d f}}, \eta_{\mathbf{d}}, \alpha, \mathbf{E}_{\mathbf{f}}\right)\right) \ldots \\
+\mathbf{C}_{\text {steamBoP }}\left(\mathbf{P}_{\text {net }}\left(\mathbf{R R}, \eta_{\text {con }}, \eta_{\mathbf{d f}}, \eta_{\mathbf{d}}, \alpha, \mathbf{E}_{\mathbf{f}}\right)\right)
\end{array}\right)
\end{aligned}
$$

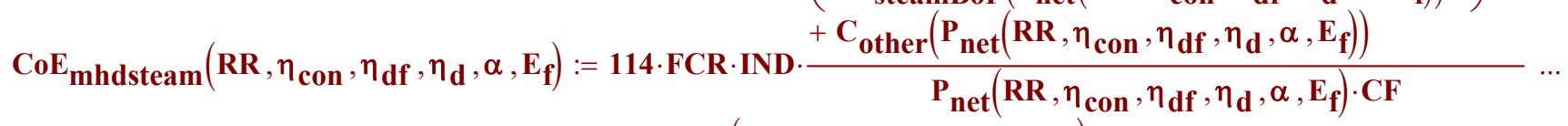

$$
\begin{aligned}
& +\mathbf{O T}_{\text {fuel }}\left(\mathbf{R R}, \eta_{\text {con }}, \eta_{\mathbf{d f}}, \eta_{\mathbf{d}}, \alpha, \mathbf{E}_{\mathbf{f}}\right) \ldots \\
& +\operatorname{OM}\left(R R, \eta_{\text {con }}, \eta_{\mathbf{d f}}, \eta_{\mathbf{d}}, \alpha, \mathbf{E}_{\mathbf{f}}\right) \\
& \text { mills/kW } \mathrm{e} \text { hr Eq } 93 \\
& \operatorname{CoE}_{A}:=\operatorname{CoE}_{\operatorname{cfar}}\left(6,0.65, \eta_{\text {dfA }}, 0.4,1.5,1\right) \quad \overline{\operatorname{CoE}_{A}=29.4} \quad \operatorname{CoE}_{\operatorname{cfar}}\left(8,0.4, \eta_{\text {dfB }}, 0.2,1.5,0.2\right)=195 \\
& \mathrm{CoE}_{\text {mhdsteam }}\left(6,0.77, \eta_{\text {dfA }}, 0.4,1.5,1\right)=36.2 \quad \mathrm{CoE}_{B}:=\operatorname{CoE}_{\text {mhdsteam }}\left(8,0.65, \eta_{\text {dfB }}, 0.2,1.5,0.2\right) \quad \operatorname{CoE}_{B}=125
\end{aligned}
$$

\section{Page 42}




\section{TABLE 10: SUMMARY}

T-lean fuel energy at ignition

Energy delivered to ablation front

Capsule implosion efficiency Overall coupling efficiency beam-to-fuel corrected for parasitic loss on ablation plasma

$\mathrm{H}_{2}$ ablation front temperature

$$
\text { Fusion yield }
$$

Driver energy

Driver efficiency

Driver electric input energy/pulse

Target gain

Fusion energy conversion eff. (lowest CoE for Demo requires $35 \%$ steam bottoming cycle to get $\mathbf{0 . 6 5}$ conversion overall)

Gross electric output (per pulse)

Net electric output per pulse, inc $5 \%$ aux

Pulse repetition rate

Net electric power

Driver direct cost

Vessel direct cost

Balance-of-Plant direct cost

Other direct costs

Cost of Electricity, inc.

targets and O\&M

\section{POWER PLANT}

$1 \mathrm{MJ}$

$\mathrm{E}_{\mathbf{d a}}(1.5,1)=2.55$

$\eta_{\mathbf{c}}(1.5,1)=0.39$

$\eta_{\text {dfA }}=0.25$

$\mathrm{T}_{\mathrm{ex}}(1.5,1)=26.3$

$Y_{f}(1.5,1)=494$

$1 \cdot \eta_{\text {dfA }}{ }^{-1}=4.06$

$\eta_{\mathbf{d A}}:=\mathbf{0 . 4}$

$1 \cdot \eta_{\mathrm{dAA}}{ }^{-1} \cdot \eta_{\mathrm{dfA}}{ }^{-1}=10.1$

$Y_{f}(1.5,1) \cdot \eta_{d f A} \cdot 1^{-1}=122$

$\eta_{\text {MHD }}:=0.65$

^| lowest CoE this case

$\mathrm{W}_{\mathrm{e}^{(0.65,1.5,1)}=278}$

$\mathrm{W}_{\text {netA }}=254$

$\mathbf{R R}_{\mathbf{A}}:=6$

$P_{\text {net } \mathrm{A}}=1522$

$C_{\text {driver }}\left(\eta_{\text {dfA }}, 1\right)=527$

$C_{\text {vessel }}(1.5,1)=59$

$C_{\text {mhdBoP }}(1522)=104$

$C_{\text {mhdBoP }}(122)+C_{\text {steamBoP }}(122)=64$

$\mathrm{C}_{\text {other }}(122)=38$

$\mathrm{CoE}_{\mathrm{B}}=125$

--> total capital < 1 B \$ for DEMO for net power and tritium production

MJ

eV

MJ

MJ

$\eta_{\mathrm{dB}}:=0.2$

$0.2 \cdot \eta_{\mathrm{dB}}{ }^{-1} \cdot \eta_{\mathrm{dfB}}{ }^{-1}=4.3 \quad \mathrm{MJ}_{\mathrm{e}}$

$Y_{f}(1.5,0.2) \cdot \eta_{d f B} \cdot 0.2^{-1}=51 \quad M_{f}$

MuMund : $=0.4$

(see Fig 3)

$\eta_{\text {MHDsteam }}:=0.65$

$\mathrm{W}_{\mathrm{e}}(0.65,1.5,0.2)=20.5 \quad \mathrm{MJ}_{\mathrm{e}}$

$\mathrm{W}_{\text {netB }}=\mathbf{1 5 . 2 1}$

$\mathbf{M J}_{\mathbf{e}}$

$\mathbf{R R}_{\mathbf{B}}:=\mathbf{8}$

$\mathrm{Hz}$

$P_{\text {netB }}=122$

$\mathrm{MW}_{\mathrm{e}}$

$C_{\text {driver }}\left(\eta_{\text {dfB }}, 0.2\right)=111$

M\$

M\$

$\mathrm{C}_{\text {other }}(\mathbf{1 5 2 2 )}=103$

$\mathrm{CoE}_{\mathrm{A}}=29.4$

->may meet affordable CoE goal for 10 billion people

As a final teaser, peek at largest T-lean 2.6 MJ fuel case: $\quad 2.6 \cdot 0.26^{-1}=10 \quad M J$ driver,

$Y_{\mathbf{f}}(1.5,2.6)=2181 \quad M J$ yield $(3 \mathrm{~m}$ radius, $10 \mathrm{~T}$ plasma chamber $), \quad P_{\text {net }}(5,0.7,0.26,0.5,1.5,2.6)=6651 \quad M W_{e}$

$$
\mathrm{CoE}_{\text {cfar }}(5,0.7,0.26,0.5,1.5,2.6)=16 \quad \mathrm{mills} / \mathrm{kW}_{\mathrm{e}} \mathrm{hr} !
$$




\section{2-D time-dependent polar (two-sided) beam requirements}

$\underline{I}_{\text {binc }}(\mathbf{r}, \mathbf{t})$ and $\rho \underline{r}_{\underline{b}}(r, t)$ for symmetric implosions

In this section we examine more closely how the goal of two-sided beam illumination might be met, and the time-dependant beam requirements, including zooming and parasitical beam loss on ablated plasma, taking into account the 2-D geometery shown in Fig. 32 below. Recent implosion calculations by John Perkins at LLNL (June 2007) indicates that one important self-consistent effect of parasitical beam heating of outgoing ablation plasma is increases in ion range (for constant ion incident energy) with increased beam intensity, and we show below this is likely the result of the electron thermal speed increased by heating beyond the ion beam speed. In the calculations on pages 28 and 29 , and equation 78, we already found one beneficial effect of beam heating in reducing ablated plasma density in the beam channel in two -sided illumination geometry (see Fig 32 below) and this is a 2-D effect for ablated plasma density reduction, but neglects range lengthening due to increases in electron temperature. On the other hand, the 2-D effect of ablated plasma expansion in a beam channel doesn't apply to Perkins' case of symmetric 1-D radial beam geometry, and moreover, beam heating of Te in a beam channel would be more concentrated in 2-D geometry. So, now we will construct a model for ion parasitic loss on ablated plasma with both Te increasing and density decreasing due to beam heating, as a guide to future HIF direct drive 2-D (two-sided) implosion calculations.

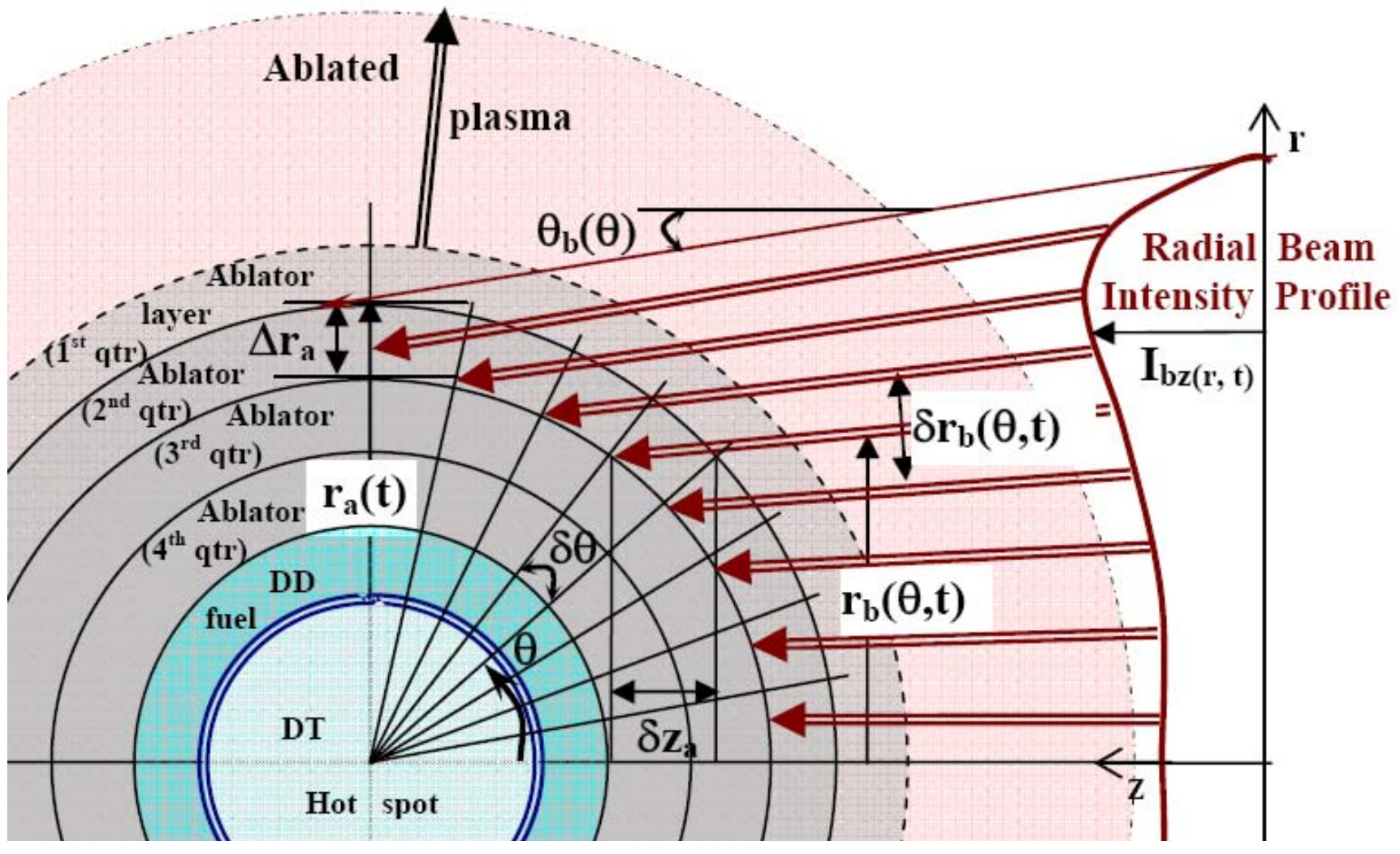

Figure 32: Geometry for two sided beam illumination of a direct drive target (only one beam side is shown). lon range is initally set $=0.25$ of initial ablator $\rho r$. The model derives beam intensity and range profiles required for spherically symmetric beam energy deposition in four layers of the ablator at four different times during the implosion. The incident beam energy is calculated to penetrate the ablation plasma in the beam channel at each time, and deposit the energy required in each ablator layer around the sphere uniformly to match the spherically symmetric ablation pressure ( $t$ ) calculated for a shaped drive pulse for a low adiabat from 1-D implosion calculations. 


$$
\begin{aligned}
& \text { uex }_{d t}:=4 \cdot \frac{10^{7}}{\ln (2)} \quad \text { uex }_{d t}=5.8 \times 10^{7} \quad \mathrm{~cm} / \mathrm{s} \text { exhaust velocity necessary to get John's implosion v } \\
& \text { Tex }_{\mathrm{dt}}:=3 \cdot 10^{-4} \cdot 2.5 \cdot \mathrm{m}_{\mathrm{h}} \cdot 1.4 \cdot \mathrm{uex}_{\mathrm{dt}}{ }^{2} \cdot 200^{-2} \cdot\left(1.6 \cdot 10^{-19}\right)^{-1} \quad \mathrm{Tex}_{\mathrm{dt}}=912 \quad \mathrm{eV} \text {. Note bremsstrahlung } \\
& \text { 0.25.0.11·0.25 }=0.007 \quad \mathrm{~g} / \mathrm{cm} 2 \text { nominal initial ion range } \quad \text { TOL }=0.001 \quad \text { DT or H ablators without } \\
& \text { han: }:=0.25 \quad \text { for DT } \quad A_{b}=40 \quad Z_{b}=18 \quad A_{t}=1 \quad Z_{t}=1 \quad T_{e}=30 \\
& \mathbf{R}_{\mathbf{b}}\left(\mathbf{E b}, \rho_{\mathbf{a}}, \mathbf{T}_{\mathbf{e}}, \mathbf{A}_{\mathbf{b}}, \mathbf{A}_{\mathbf{t}}, \mathbf{Z}_{\mathbf{b}}, \mathbf{Z}_{\mathbf{t}}\right):=\rho_{\mathbf{a}}{ }^{-1} \cdot \int_{\mathbf{E b}}^{0.02 \cdot \mathrm{Eb}} \mathbf{d E}_{\mathbf{d} \rho \mathbf{x}}\left(\rho_{\mathbf{a}}, \mathbf{E}, \mathbf{T}_{\mathbf{e}}, \mathbf{A}_{\mathbf{b}}, \mathbf{A}_{\mathbf{t}}, \mathbf{Z}_{\mathbf{b}}, \mathbf{Z}_{\mathbf{t}}\right)^{-1} \mathbf{d E} \quad \underset{\text { Eq. } 94}{\text { range, }} \mathbf{c m} \\
& \mathbf{R}_{\mathbf{b}}\left(5 \cdot 10^{7}, \rho_{\mathbf{a}}, 30, \mathbf{A}_{\mathbf{b}}, 2.5, \mathbf{Z}_{\mathbf{b}}, 1\right)=0.007 \quad \mathrm{~cm} \text {-close agreement with John's inital ion range } \\
& \rho_{\mathbf{a}} \cdot \mathbf{R}_{\mathbf{b}}\left(5 \cdot 10^{7}, \rho_{\mathbf{a}}, 30, \mathbf{A}_{\mathbf{b}}, 2.5, \mathrm{Z}_{\mathbf{b}}, 1\right)=0.002 \quad \text { rho-r range in } \mathrm{g} / \mathrm{cm} 2
\end{aligned}
$$

Table 11: Range vs temperature. Note how the ion beam it $:=1 . .12 \quad T_{i t}:=2^{\text {it }-1} \quad$ increases Te during the pulse in this table!

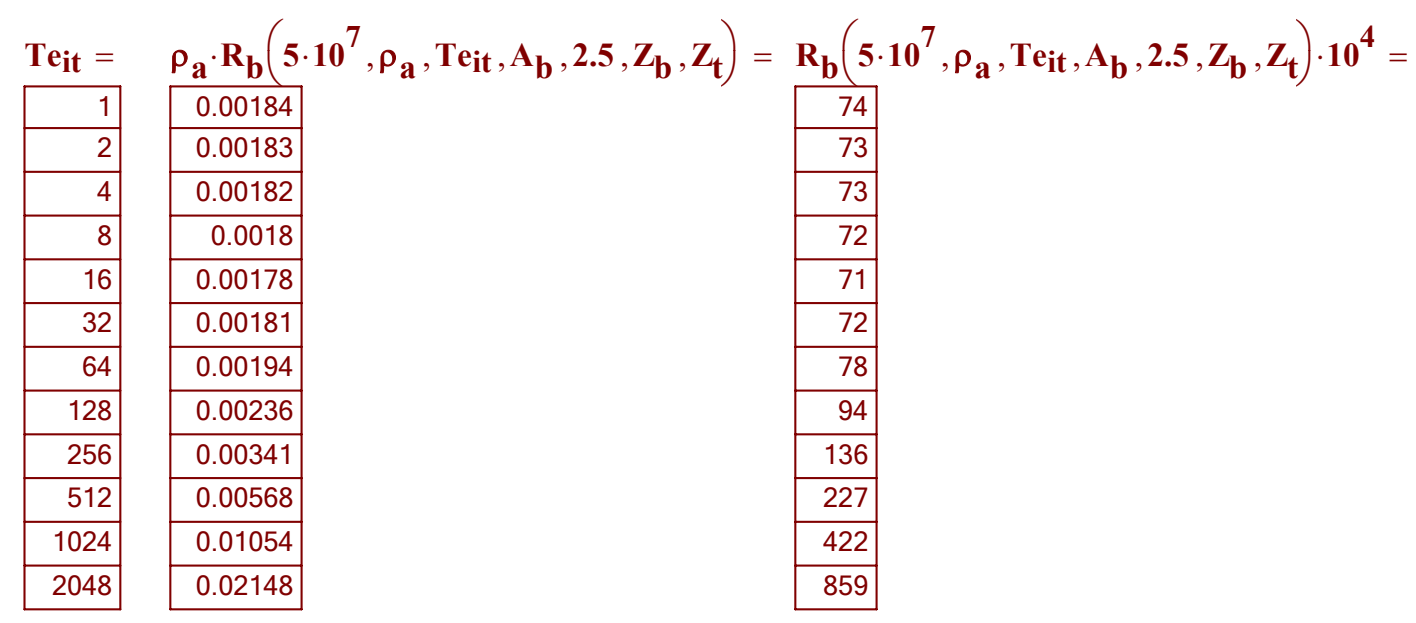

$\mathrm{eV} \quad \mathrm{g} / \mathrm{cm}^{2} \quad$ range in microns

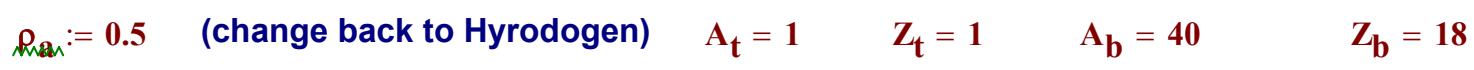

$$
\begin{array}{ll}
E_{b f}\left(\rho_{a}, 0.0008,0.01,30, A_{b}, A_{t}, Z_{b}, Z_{t}\right)=4.94 \times 10^{7} & \text { Small } 1 \text { MJ DT case (Perkins) } \\
E_{b f}\left(\rho_{a}, 0.004,0.01,30, A_{b}, A_{t}, Z_{b}, Z_{t}\right)=2.03 \times 10^{8} & 5 M J \text { T-lean case }
\end{array}
$$

We have already estimated the radial expansion of plasma in the beam channel (see Eq 77 page 29), from which we can infer an ablation plasma electron temperature due to beam heating:

$$
\text { Tea }_{k}:=\frac{0.5}{2} \cdot m_{h} \cdot 10^{-3} \cdot\left(\text { vaperp }_{k}\right)^{2} \cdot \frac{10^{-4}}{\mathrm{e}}
$$

Eq 95.

See plot on next page, Fig. 34 . 


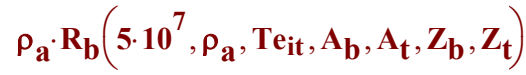

$$
\begin{aligned}
& \overline{\frac{\rho_{\mathbf{a}}}{10} \cdot \mathbf{R}_{\mathbf{b}}}\left(\mathbf{5 \cdot 1 0}, \frac{\rho_{\mathbf{a}}}{\mathbf{1 0}}, \mathbf{T e}_{\mathbf{i t}}, \mathbf{A}_{\mathbf{b}}, \mathbf{A}_{\mathbf{t}}, \mathbf{Z}_{\mathbf{b}}, \mathbf{Z}_{\mathbf{t}}\right) \\
& \rho_{\mathbf{a}} \cdot \mathbf{R}_{\mathbf{b}}\left(2 \cdot 10^{8}, \rho_{\mathrm{a}}, \mathrm{Te}_{\mathrm{it}}, \mathbf{A}_{\mathrm{b}}, \mathbf{A}_{\mathrm{t}}, \mathbf{Z}_{\mathrm{b}}, \mathbf{Z}_{t}\right) \\
& \overline{\frac{\rho_{\mathbf{a}}}{10} \cdot \mathbf{R}_{\mathbf{b}}}\left(2 \cdot 10^{8}, \frac{\rho_{\mathbf{a}}}{10}, \mathbf{T e}_{\mathbf{i t}}, \mathbf{A}_{\mathbf{b}}, \mathbf{A}_{\mathbf{t}}, \mathbf{Z}_{\mathbf{b}}, \mathbf{Z}_{\mathbf{t}}\right) \\
& \begin{array}{r}
0.0 \\
1 \cdot 10^{-3}
\end{array} \\
& \text { ) }
\end{aligned}
$$

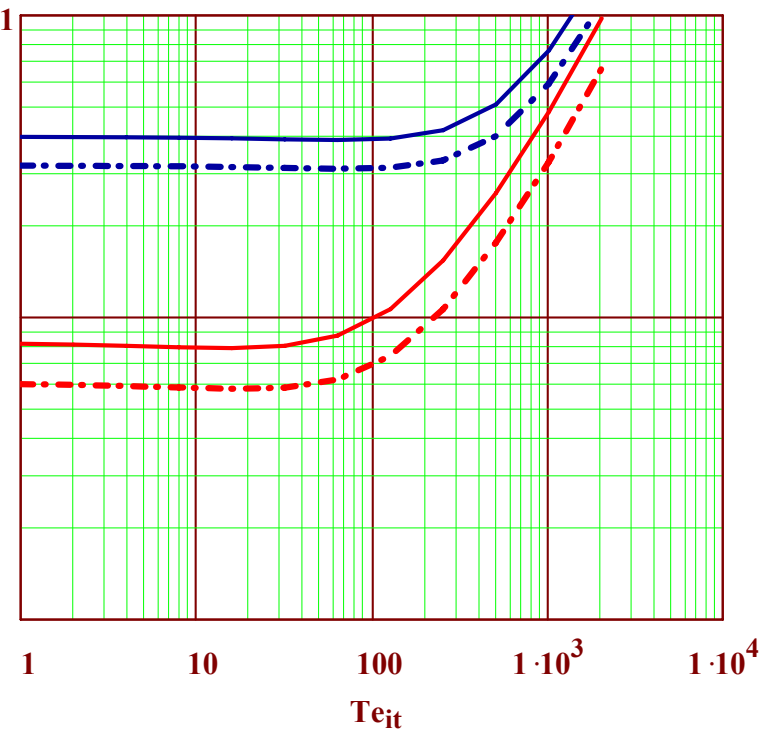$$
1 \cdot 10^{-4}
$$

$\mathrm{Te}_{\text {it }}$

\author{
<- 5MJ Reactor \\ case@ \\ $200 \mathrm{MeV}$ Ar, \\ 0.5 and 0.05 \\ $\mathrm{g} / \mathrm{cm}^{2}$ density
}

<- 1 MJ Demo

@ 50 MeV Ar,

0.5 and 0.05

$\mathrm{g} / \mathrm{cm}^{2}$ density

-->Ablated

plasma Te

Figure 33: Ion range versus plasma temperature. Note range first shortens slightly as Te goes from $1 \mathrm{eV}$ to $100 \mathrm{eV}$, and then increases several fold with Te up to $2 \mathrm{keV}$. There is also a range shortening $\left(20 \% \log \lambda_{D}\right.$ effect) at $10 X$ lower density of ablated plasma, partially offsetting the effect of ablated plasma expansion.

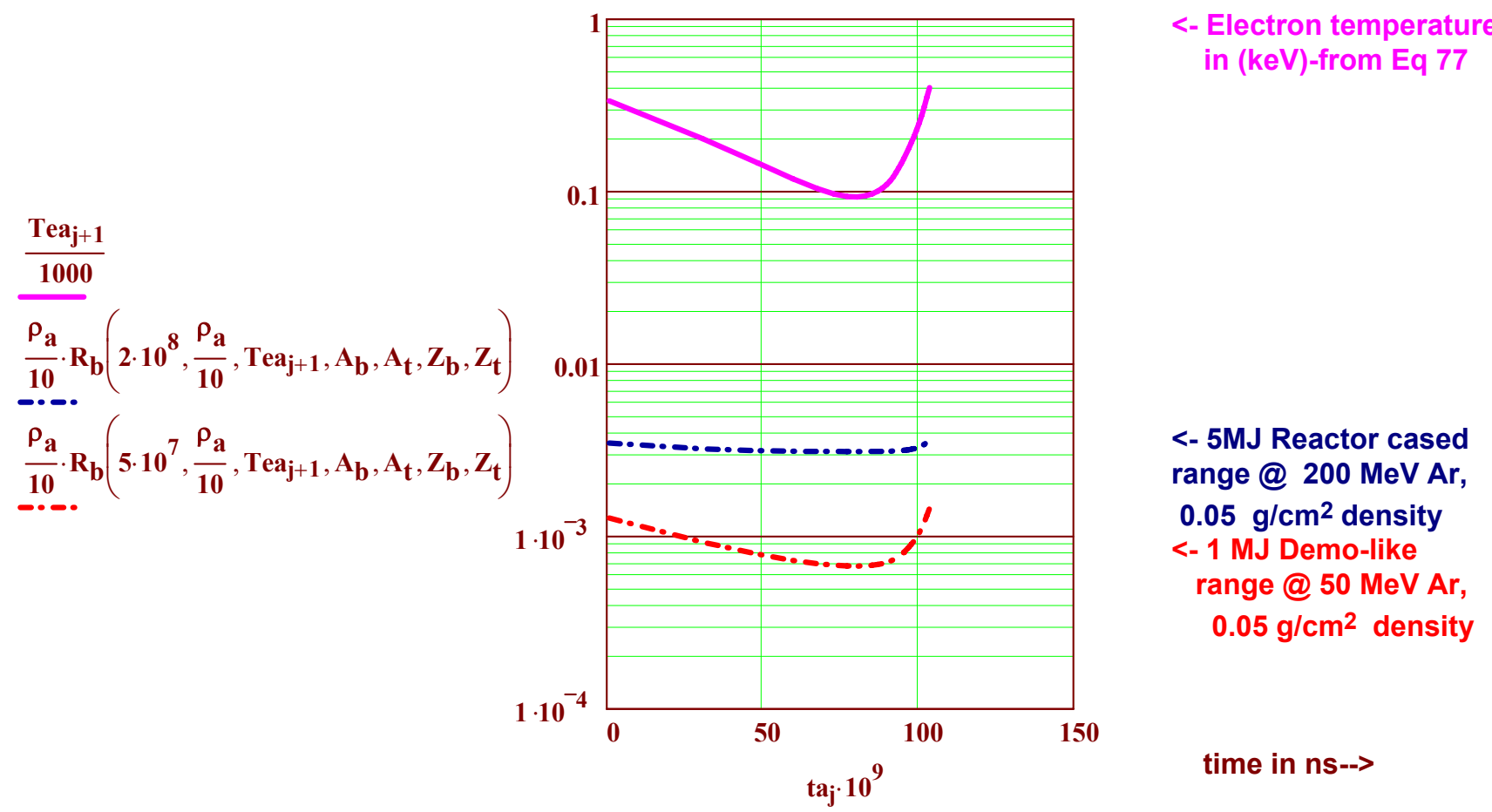

Figure 34: Ion ranges and Te during Case A implosions for constant energy $200 \mathrm{MeV}$ and 50 MeV Argon ion beams. The beam heating effect on Te and range is most pronounced for the lower velocity $50 \mathrm{MeV}$ beams. Early in time during the foot of the implosion, Te and range both decrease because ablated mass increases faster than beam input to it minus expansion cooling, but then later, net beam input exceeds ablation mass rates ( $\mathrm{J} / \mathrm{g}$ goes up) in the ramp up to peak power, when Te and range again increase. This may explain qualitatively why, in John Perkins implosion calculations, the beam $\mathbf{5 0} \%$ deposition radius first migrates away from the imploding shell, up until the big ramp up to peak power begins, after which the $50 \%$ absorption point migrates back to the dense shell. Quantitatively, this range variation should be larger in John Perkins 1 MJ example than this at lower beam intensity and Te for a 4 MJ case A target. 
lon beam requirements for Case A implosions with polar (two-sided) beam illumination.

With initial ion range set equal to one fourth of the initial ablator $\rho$ r, determine required $\quad$ js $:=1 . .4$ two sided beam input intensity and range at four times $t_{j s}$, when the ablator mass is 1 ,

$3 / 4,1 / 2$, and $1 / 4$ of initial mass. Determine beam intensity and range $\left[I_{b z}(r, t)\right.$ and $\left.\rho r_{b}(r, t)\right]$ is := $1 . .9$

for beam deposition uniform in $\theta$ and in $\mathrm{J} / \mathrm{g}$ (Fig. 32) into 8 polar segments of angles $\theta_{\text {is }}$

and width $\pi / 16$. Account for beam attenuation in ablated plasma at each time and $\theta$.

$\theta_{\text {is }}:=\left(\frac{\pi}{16}\right) \cdot($ is -1$)$

Work for Case A power plant (page 25) and then for Case B Demo (page 32).

1st quarter ablator mass $\quad \delta \mathrm{MaA}_{\mathrm{js}}:=(115-23) \cdot\left(4 \cdot 10^{3}\right)^{-1} \quad \delta \mathrm{MaA}_{1}=0.023 \quad \mathrm{~g}$, @ density $\quad \rho \mathrm{AA}_{1}:=\rho_{\mathrm{Ho}}$

1st qtr outer radius $\quad \operatorname{raA} 0:=0.71 \quad \mathrm{~cm}$, ablated over time interval $\quad \mathrm{tA}_{1}:=0 \quad \delta \mathrm{tA}_{1}:=81 \cdot 10^{-9} \mathrm{~s}$

Ist qtr inner radius $\quad \operatorname{raA}_{1}:=\left[\mathrm{raA}^{3}-\left(\frac{3 \cdot \delta \mathrm{MaA}_{1}}{4 \cdot \pi \cdot \rho \mathrm{aA}_{1}}\right)\right]^{0.333} \quad \mathrm{~cm} \quad \mathrm{raA}_{1}=0.67 \quad \mathrm{~cm} \quad \delta \mathrm{raA}_{1}:=\mathrm{raA}-\mathrm{raA}_{1}$

1st qtr layer thickness $\quad \delta \mathrm{raA}_{1}=0.038 \quad \mathrm{~cm}$. Radial KE/exhaust energy efficiency $\quad \eta_{\text {ex }}(1.5,1)=0.61$

1st qtr drive energy $\quad \operatorname{EdA}_{1}:=0.385 \cdot 10^{6} \cdot \eta_{\mathrm{ex}}(1.5,1)^{-1} \quad \mathrm{EdA}_{1}=6.4 \times 10^{5} \quad$ J. Power: $\quad \operatorname{PdA}_{1}:=\operatorname{EdA}_{1} \cdot\left(\delta \mathrm{\delta AA}_{1}\right)^{-1}$

$\operatorname{PdA}_{1} \cdot 10^{-12}=7.85 \quad$ TW. 1st qtr energy density $\quad \operatorname{WdA}_{1}:=\operatorname{EdA}_{1} \cdot\left(\delta \mathrm{MaA}_{1}\right)^{-1} \quad \mathrm{WdA}_{1}=2.8 \times 10^{7} \quad \mathrm{~J} / \mathrm{g}$

Ist quarter ablation front pressure $\quad \mathrm{WdA}_{1} \cdot \rho \mathrm{aA}_{1} \cdot 10^{6} \cdot 2^{-1} \cdot 10^{-11}=13.8 \quad \mathrm{MB} \quad \mathrm{WdA} \cdot \frac{\mathrm{m}_{\mathrm{h}}}{10 \cdot \mathrm{e}}=29 \quad \mathrm{eV}$

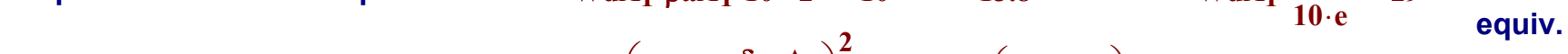

1 st qtr shell volume $\quad \delta \mathrm{VaA}_{1}$, is $:=2 \cdot \pi \cdot\left(\operatorname{raA}_{1}+\frac{\delta \mathrm{raA}_{1}}{2}\right)^{2} \cdot \delta \mathrm{raA}_{1} \cdot \sin \left(\theta_{\text {is }}+\frac{\pi}{32}\right) \cdot \frac{\pi}{16} \quad \mathrm{~cm}^{3}$

2. $\sum_{\text {is }=1}^{8} \delta \mathrm{VaA}_{1}$, is $=0.229 \quad \mathrm{~cm}^{3} \quad \frac{4}{3} \cdot \pi \cdot\left[\left(\mathrm{raA}_{1}+\delta \mathrm{raA}_{1}\right)^{3}-\left(\mathrm{raA}_{1}\right)^{3}\right]=0.228 \quad$ Shell volume checks OK!

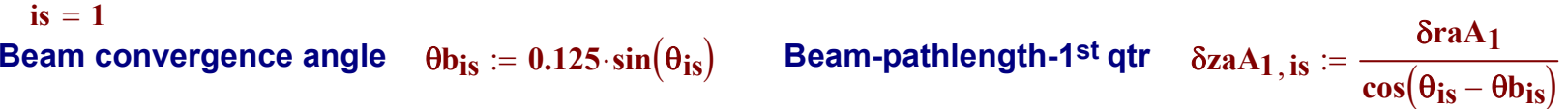

Beam range $\quad \operatorname{praA}_{1}$, is $:=\rho \mathrm{aA}_{1} \cdot \delta \mathrm{zaA}_{1}$, is $\quad$ \& energy $\mathbf{E b o}_{1}$, is $:=\mathbf{E}_{\mathbf{b f}}\left(\rho \mathrm{aA}_{1}, \rho \operatorname{raA} \mathbf{A}_{1}\right.$, is $\left., 0.01,28, \mathbf{A}_{\mathbf{b}}, \mathbf{A}_{\mathbf{t}}, \mathbf{Z}_{\mathbf{b}}, \mathbf{Z}_{\mathbf{t}}\right)$

Incident beam radius vs polar angle $\theta \quad \mathrm{rbA}_{1}$, is $:=\operatorname{raA}_{1} \cdot \sin \left(\theta_{\mathrm{is}}+\frac{\pi}{32}\right) \quad \operatorname{rbA}_{1}, 9:=\operatorname{raA} 0+\delta \mathrm{zaA}_{1}, 9 \cdot \theta \mathrm{bag}$

Beam illumination width (cm) per $\theta$ increment $\quad \delta \mathrm{rbA}_{1}$, is $:=\left(\operatorname{raA}_{1}+\frac{\delta \mathrm{raA}_{1}}{2}\right) \cdot \sin \left(\theta_{\text {is }}+\frac{\pi}{16}\right)-\operatorname{raA} \cdot \sin \left(\theta_{\text {is }}\right)$

Beam deposition intensity $\left(r_{b}\right) \quad \operatorname{IdA} A_{1}$, is := $\operatorname{WdA}_{1} \cdot \delta \operatorname{VaA}_{1}$, is $\cdot \rho \mathrm{aA}_{1} \cdot\left(\delta \mathrm{tA}_{1} \cdot 2 \cdot \pi \cdot \operatorname{rbA} A_{1} \text {, is } \cdot \delta \mathrm{rbA}_{1}, \text { is }\right)^{-1} \quad \operatorname{IdA} A_{1}, 9:=0$

Table 12: 2-D polar drive requirements for Case A, $1^{\text {st }}$ quarter ablation period. $\quad$ Ebo1,9:= Ebo1,8

\begin{tabular}{|c|c|c|c|c|c|}
\hline$\theta_{\mathrm{is}}=$ & $\operatorname{rbA}_{1, \text { is }}=$ & $\delta z A_{1}$, is $=$ & $\operatorname{\rho raA} A_{1}$, is $=$ & $\operatorname{Id} A_{1}$, is $\cdot 10^{-12}=$ & Ebo1, is $\cdot 10^{-6}=$ \\
\hline 0 & 0.07 & 0.04 & 0.0038 & 1.3 & 219 \\
\hline 0.2 & 0.2 & 0.04 & 0.0039 & 1.4 & 222 \\
\hline 0.39 & 0.32 & 0.04 & 0.004 & 1.4 & 229 \\
\hline 0.59 & 0.43 & 0.04 & 0.0044 & 1.6 & 243 \\
\hline 0.79 & 0.52 & 0.05 & 0.005 & 1.8 & 265 \\
\hline 0.98 & 0.59 & 0.06 & 0.006 & 2.3 & 301 \\
\hline 1.18 & 0.64 & 0.08 & 0.0078 & 3.2 & 362 \\
\hline 1.37 & 0.67 & 0.12 & 0.0121 & 5.7 & 483 \\
\hline 1.57 & 0.75 & 0.31 & 0.0305 & 0 & 483 \\
\hline $\begin{array}{l}\text { Polar } \\
\text { angle (rad) }\end{array}$ & $\begin{array}{l}\text { Beam } \\
\text { radius }(\mathrm{cm})\end{array}$ & $\begin{array}{l}\text { Shell } \\
\text { z depth }(\mathrm{cm})\end{array}$ & $\begin{array}{l}\text { Beam Page } 47 \\
\text { range } \mathrm{g} / \mathrm{cm}^{2}\end{array}$ & $\begin{array}{l}\text { Beam } \\
\text { Intensity } \mathrm{TW} / \mathrm{cm}^{2}\end{array}$ & $\begin{array}{l}\text { Argon Beam } \\
\text { Energy }(\mathrm{MeV})\end{array}$ \\
\hline
\end{tabular}


$\sum_{\text {is }=1}^{8} \delta \operatorname{rbA}_{1}$ is $=0.778 \quad \mathrm{~cm} \quad 2 \cdot \sum_{\text {is }=1}^{8}\left(\mathrm{WdA}_{1} \cdot \delta \mathrm{VaA}_{1}\right.$, is $\left.\cdot \rho \mathrm{aA}_{1}\right)=6.3 \times 10^{5} \quad$ J-Energy Checks-OK! $\sum_{\text {is }=1}^{8}\left[2 \cdot \pi \cdot\left(\operatorname{rbA}_{1}\right.\right.$, is $) \cdot \delta \mathrm{rbA} 1$, is $]=1.77 \quad \mathrm{~cm}^{2} \quad \pi \cdot \mathrm{raA0}^{2}=1.58 \quad \mathrm{~cm}^{2}$. OK as converging beam sees $>2 \pi$

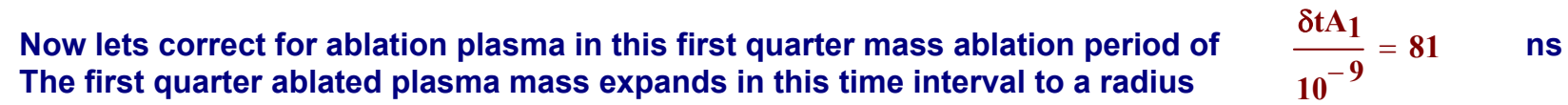
$\underset{\text { uimpA }}{1}:=2 \cdot 10^{6} \quad \mathrm{~cm} / \mathrm{s} \quad \operatorname{rpA}_{1}:=\operatorname{raA} 0+\left(\mathrm{u}_{\mathrm{ex}}(1.5,1)-\mathrm{uimpA}_{1}\right) \cdot \delta \mathrm{tA}_{1} \quad \mathrm{~cm} \quad \mathrm{rpA}_{1}=2.03 \quad \mathrm{~cm}$ average expansion velocity and to a mass density $\quad \operatorname{ppA}_{1}:=\delta \mathrm{MaA}_{1} \cdot\left[\frac{4}{3} \cdot \pi \cdot\left[\left(\operatorname{rpA}_{1}\right)^{3}-\mathrm{raA0}^{3}\right]\right]^{-1} \quad{\rho p A_{1}}^{-1}=6.8 \times 10^{-4} \quad \mathrm{~g} / \mathrm{cm} 3$ and to a rho-r $\quad \operatorname{prpA}_{1}:=\rho \mathrm{pA}_{1} \cdot\left(\operatorname{rpA}_{1}-\mathrm{raA} 0\right) \quad \rho \mathrm{prA}_{1}=9.03 \times 10^{-4} \mathrm{~g} / \mathrm{cm}^{2}, \sim 20 \%$ of the ave first quarter beam range.

(Henceforth, to avoid confusion. we switch to subscript "p" to denote ablated plasma quantities, leaving the subscript "a" to apply to the dense remaining ablator shell.

$$
\begin{aligned}
& E b=3 \times 10^{8} \quad A_{b}=40 \quad Z_{b}=18 \quad A_{t}=1 \quad Z_{t}=1 \quad n_{p}:=\rho p A_{1} \cdot m_{h}{ }^{-1} \quad n_{p}=4.09 \times 10^{20} \\
& \tau_{p}:=6.4 \cdot 10^{17} \cdot 0.1^{1.5} \cdot\left(4 \cdot 10^{26} \cdot 7\right)^{-1} \quad \tau_{p}=7.23 \times 10^{-12} \quad \text { s.-->ablation plasma is very collisional }
\end{aligned}
$$

Now lets solve for the initial incident beam energy, which first slows down partially in the ablation plasma to an intermediate energy $\mathrm{Eb}_{\mathrm{p}}$, then enters the dense remaining dense ablator shell where it gives up its remaining energy wihtin the specified shell $\rho_{a} \Delta r_{a}$

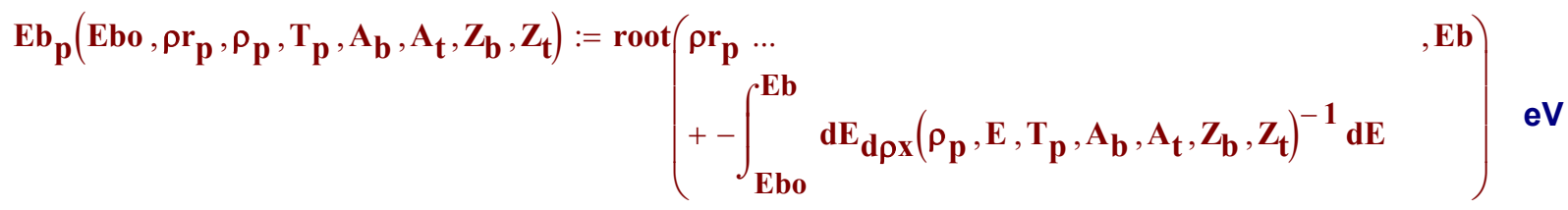
Required ion range neglecting ablation plasma: $\quad \operatorname{Eb}_{\mathbf{p}}\left(5 \cdot 10^{8}, \rho \operatorname{\rho rp} \mathrm{A}_{1}, \rho p \mathrm{~A}_{1}, 30, \mathrm{~A}_{\mathrm{b}}, \mathrm{A}_{t}, \mathrm{Z}_{\mathrm{b}}, \mathrm{Z}_{\mathrm{t}}\right)=4.69 \times 10^{8}$
Required ion range with ablation plasma

$$
\begin{aligned}
& \text { Initial guess } \quad \mathbf{E b}:=10^{9} \\
& \mathbf{E}_{\mathbf{b o}}\left(\rho r_{\mathbf{p}}, \rho r_{\mathbf{a}}, \rho_{\mathbf{p}}, \rho_{\mathbf{a}}, \mathbf{T}_{\mathbf{p}}, \mathbf{T}_{\mathbf{a}}, \mathbf{A}_{\mathbf{b}}, \mathbf{A}_{\mathbf{t}}, \mathbf{Z}_{\mathbf{b}}, \mathbf{Z}_{\mathbf{t}}\right):=\operatorname{root}\left(\rho \mathbf{r}_{\mathbf{a}} \ldots\right. \\
& +-\int_{\mathbf{E} b_{p}\left(\mathbf{E b}, \rho r_{p}, \rho_{p}, T_{p}, A_{b}, A_{t}, Z_{b}, Z_{t}\right)}^{0.02 \cdot E b} \mathbf{d E}_{\mathbf{d} \rho \mathbf{x}}\left(\rho_{\mathbf{a}}, \mathbf{E}, \mathbf{T}_{\mathbf{a}}, \mathbf{A}_{\mathbf{b}}, \mathbf{A}_{\mathbf{t}},\right.
\end{aligned}
$$


Table 13: required incident beam energies, Case A, 1st qtr ablation

With ablation plasma loss (1st qtr) assuming $T_{p}=80 \mathrm{eV}$

\begin{tabular}{|r|}
$\mathbf{E}_{\text {bo }}(\boldsymbol{\rho} \mathbf{p} \mathbf{A}$ \\
\hline $2.7 \cdot 108$ \\
\hline $2.73 \cdot 108$ \\
\hline $2.79 \cdot 108$ \\
\hline $2.91 \cdot 108$ \\
\hline $3.12 \cdot 108$ \\
\hline $3.44 \cdot 108$ \\
\hline $4.01 \cdot 108$ \\
\hline $5.16 \cdot 108$ \\
\hline $8.8 \cdot 108$ \\
\hline
\end{tabular}

Without ablation plasma (1st qtr)

Ebo1, is =
\begin{tabular}{|c|}
\hline $2.19 \cdot 108$ \\
\hline $2.22 \cdot 108$ \\
\hline $2.29 \cdot 108$ \\
\hline $2.43 \cdot 108$ \\
\hline $2.65 \cdot 108$ \\
\hline $3.01 \cdot 108$ \\
\hline $3.62 \cdot 108$ \\
\hline $4.83 \cdot 108$ \\
\hline $4.83 \cdot 108$ \\
\hline
\end{tabular}

More exact beam energy after passing through ablation plasma (very close to the simpler form of Ebo):

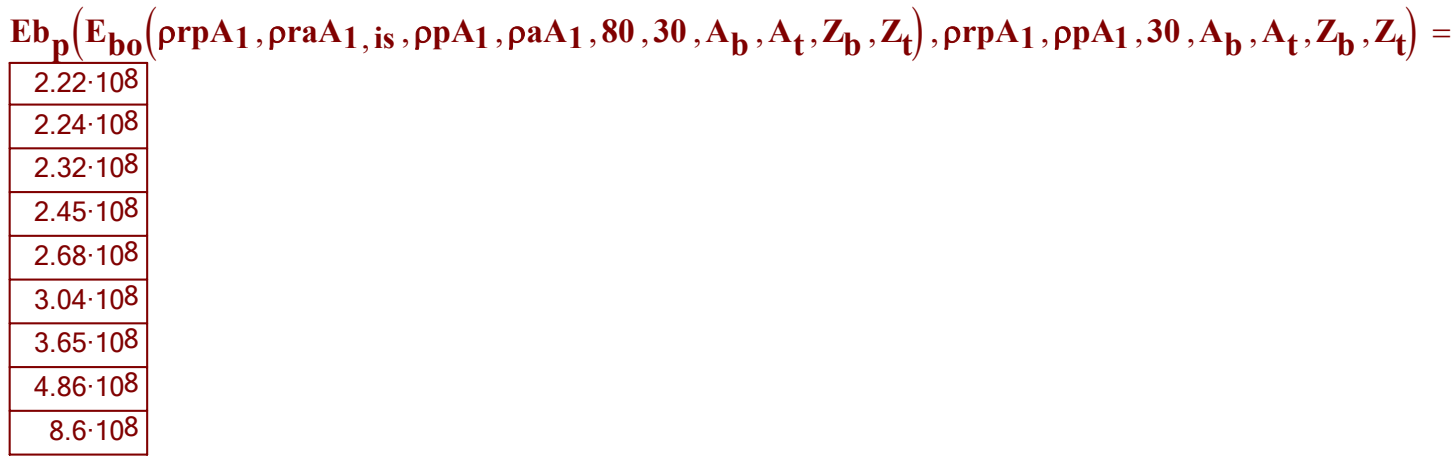

Lets make a first order correction to the exhaust plasma temperature, assuming half of the incremental beam energy deposited into the ablation plasma mass within the beam channel

$$
\triangle \operatorname{EbpA}_{1} \text { is }:=\left(\mathbf{E}_{\mathbf{b o}}\left(\operatorname{\rho rpA_{1}}, \operatorname{\rho raA}_{1}, \text { is }, \rho p A_{1}, \rho A_{1}, 80,30, \mathbf{A}_{\mathbf{b}}, \mathbf{A}_{\mathbf{t}}, \mathbf{Z}_{\mathbf{b}}, \mathbf{Z}_{\mathbf{t}}\right)-\mathbf{E b o 1}_{1} \text {, is }\right) \cdot\left(\mathbf{E b o 1}_{1} \text {, is }\right)^{-1}
$$

goes into increased hydro motion (both radial and transverse), the other half into incremental thermal energy $3 \Delta T_{p}$, and for the moment lets assume the ablated plasma density does not change; then we have

$$
\begin{aligned}
\operatorname{TpA}_{1, \text { is }}:= & \frac{\operatorname{IdA}_{1, \text { is }} \cdot \delta \mathrm{tA}_{1} \cdot \Delta \mathrm{EbpA}_{1}, \text { is }}{\rho \mathrm{mpA}_{1}} \cdot \frac{\mathbf{m}_{\mathbf{h}}}{6 \cdot \mathbf{e}} \ldots \\
& +\mathbf{T}_{\mathbf{e x}}(1.5,1)
\end{aligned}
$$$$
\text { Table 14: Effects of beam heating of ablation plasma as a }
$$
funtion of polar angle $\theta$ in the beam channel

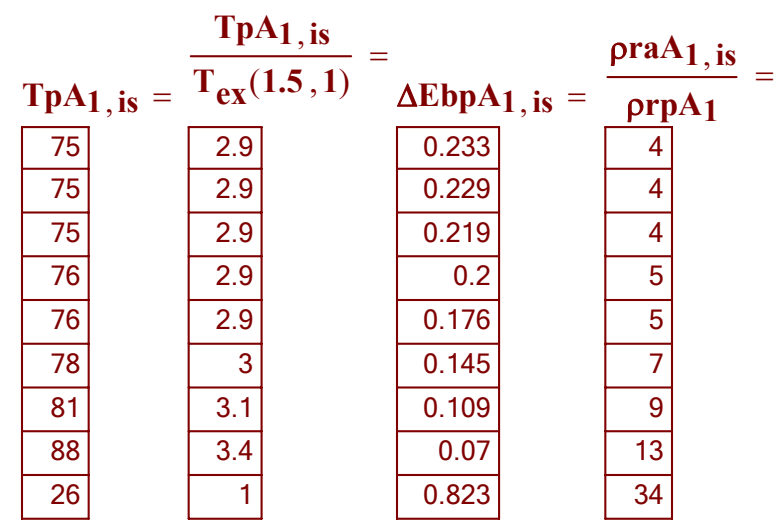

One can see in this table that the ablation plasma is substantially heated (Te increases several-fold) during the 1st qtr of the pulse, but not enough to significantly increase ion range with ve>vi. 
The next question to ask is, does the heating (pressurization) cause significant enhanced expansion in the shorter transverse to the beam channel (polar axis) direction? One estimate is the fractional radial expansion of ablated plasma possible in the 1st qtr time of $\delta \mathrm{tA}$, following eq77:

$\operatorname{vperpA}_{1}$, is $:=10^{2} \cdot \sqrt{\frac{5 \cdot\left[\left(\mathrm{TpA}_{1}, \text { is }\right)-\mathrm{T}_{\mathrm{ex}}(1.5,1)\right] \cdot \mathrm{e}}{3 \cdot \mathrm{m}_{\mathrm{h}} \cdot 10^{-3} \mathrm{~cm} / \mathrm{s}}}$ Table 15: Displacement of ablated plasma by beam heating

Table 15 showing vperp* $\delta t / \delta$ rb $\gg>1$ means that beam heating of ablation plasma will reach pressure equilibrium locally within the beam channel very quickly, and vperp* $\delta$ t/ $\mathrm{ra} \sim 1$ means significant expansion transverse to the polar axis over the whole channel, justifying allocating half the incremental beam energy input going into hydro motion. The first effect of local pressure balance means the local

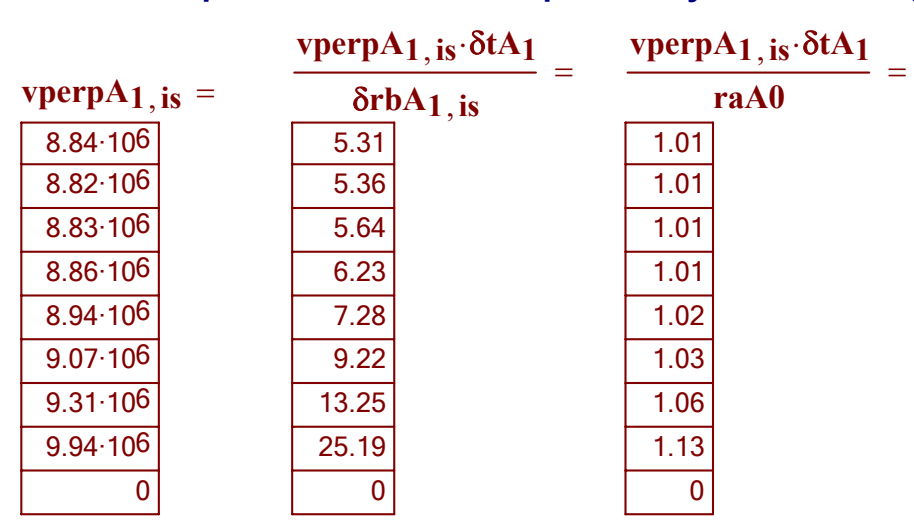

ablation plasma density will be depressed inversely with the local increase in beam temperature. The second effect will reduce the overall pressure withing the beam channel by roughly a factor of $(1+v p e r p * \delta t / r a)^{\wedge}-1$ (The net $T_{p}$ will stay roughly the same as beam energy $d E / d x$ transfer as "per electron"remains rougly the same. Using "pressure balance" and channel expansion factors, the corrected ablation plasma densities and associated rho-r's are reduced by beam heating are estimated by

$$
\begin{aligned}
& \rho_{\mathbf{p A}_{1, \text { is }}}:=\rho p \mathbf{A}_{1} \cdot\left(\frac{\operatorname{TpA}_{1,1}}{\operatorname{TpA}_{1}, \text { is }}\right) \cdot\left(1+\frac{\operatorname{vperpA_{1},\text {is}} \cdot \delta \mathbf{t A}_{1}}{\operatorname{raA} 0}\right)^{-2} \\
& \rho_{\mathbf{p A}_{1,9}}:=\rho \mathbf{p A}_{1} \\
& \text { and } \quad \operatorname{\rho r}_{\mathbf{p A}_{1, \text { is }}}:=\operatorname{\rho rpA_{1}} \cdot\left(\frac{\operatorname{TpA}_{1,1}}{\operatorname{TpA}_{1}, \text { is }}\right) \cdot\left(1+\frac{\operatorname{vperpA_{1},\text {is}} \cdot \delta \mathbf{t A}_{1}}{\operatorname{raA} 0}\right)^{-2} \\
& \rho \mathbf{p A}_{1,9}:=\rho \operatorname{prpA_{1}}
\end{aligned}
$$

We can now plot all these adjusted beam requirements versus beam radius in the next Figure 35 . The corrected incident beam energy requirement adjusted for heated ablation plasma loss is

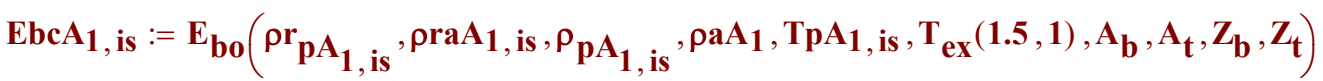

$$
\begin{aligned}
& \text { Page } 50 \\
& \operatorname{EbcA1}_{1,9}:=\operatorname{EbcA}_{1}, 8
\end{aligned}
$$




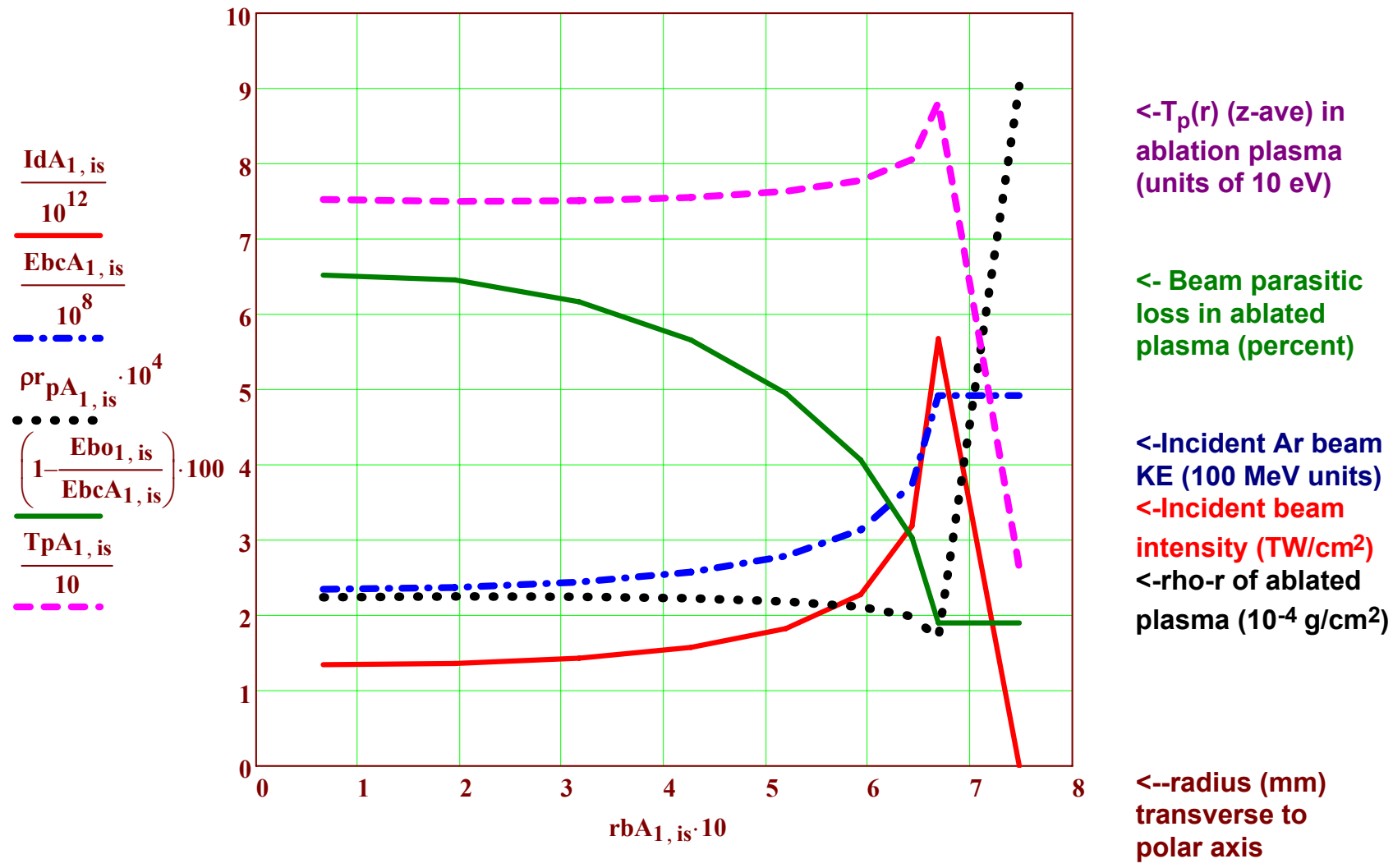

Figure 35: Plots of polar beam drive intensity (TW/ $\mathrm{cm}^{2}$, one of two sides), incident Ar beam energy (in $100 \mathrm{MeV}$ units), rho-r of ablated plasma column density $\left(10^{-4} \mathrm{~g} / \mathrm{cm}^{2}\right.$ units) (dotted black line), percent beam loss in ablated plasma, and the temperature $T_{p}$ of the ablation plasma(units of $10 \mathrm{eV}$ ), as

functions of radius in the beam channel, transverse to the polar axis near the target, during the first quarter of the ablation drive pulse, (the foot part of the pulse) for the large T-lean target case A (see Figs 19 to 23 above for case A details). Note required beam intensity is sharply higher (peak is $\sim 4 \mathrm{X}$ intensity on axis) in the beam channel "rim", as expected due to the polar drive geometry shown in Fig. 32. Also, note the local ablation temperature $\left(T_{p}\left(r_{b}\right)\right.$ increases with the beam intensity, resulting in pressurization digging a "hole" in ablated plasma rho-r just in the annulus through which most beam energy is delivered, reducing parasitic beam loss. This beneficial effect will increase later in the drive.

$$
\begin{aligned}
& \begin{array}{l}
\text { 1st qtr beam input inc } \\
\text { loss on ablation plasma }
\end{array} \quad \operatorname{EdcA}_{1}:=\sum_{\text {is }=1}^{8}\left[\frac{\operatorname{EbcA}_{1} \text {, is }}{\operatorname{Ebo}_{1}, \text { is }} \cdot 2 \cdot\left(\operatorname{WdA}_{1} \cdot \delta \operatorname{VaA}_{1}, \text { is } \cdot \rho A_{1}\right)\right] \quad \quad \operatorname{EdcA}_{1}=6.6 \times 10^{5} \\
& \text { Neglecting beam ablation loss } \quad \operatorname{EdA}_{1}=6.36 \times 10^{5} \quad-\rightarrow \text { fractional loss }\left(\operatorname{EdcA}_{1}-\operatorname{EdA}_{1}\right) \cdot\left(\operatorname{EdcA}_{1}\right)^{-1}=0.037
\end{aligned}
$$

Fig. 35 shows a key feature of polar drive geometry-the local peaking of beam intensity and locally higher beam ion energy in the "rim" of the beam channel driving the limb of the ablator shell. This ideal beam variation provides symmetric ablation drive for a spheical implosion, but may prove difficult to achieve in practice, and so further work will explore ways to relax the locally-sharp, beam intensity "rim":

(a) Most important, add appropriate $\Delta \mathrm{ra}(\theta)$ capsule ablator shimming to accept a more uniform beam profile;

(b) Allow 20\% low mode-P2 asymmetries at large rho-r fuel (Steve Slutz at San Ramon IFE meeting);

(c) Possibly in conjuction with (a), overdrive the foot intensity at the rim for early 5 to $20 \%$ P2 prolate asymmetry with beam spill beyond the limb (small drive energy penalty, and then under-drive the rim later; (d) If ignition still fails in a too-asymmetric implosion, then add a powerful late shock. 
Polar drive parameters for Case A, 2nd quarter of ablation drive

$2^{\text {nd }}$ quarter ablator mass $\quad \delta \mathrm{MaA}_{2}=0.023 \quad \mathrm{tA}_{2}:=\delta \mathrm{tA}_{1} \quad \mathrm{tA}_{2}=8.1 \times 10^{-8} \quad \mathrm{~g}$, @ density $\rho \mathrm{aA}_{2}:=3 \cdot \rho_{\mathrm{Ho}}$ $2^{\text {nd }}$ qtr outer radius $\quad \operatorname{raA1}:=0.61 \quad \mathrm{~cm}$, ablated over time interval $\quad \delta \mathrm{tA}_{2}:=11.95 \cdot 10^{-9} \mathrm{~s}$

2nd qtr inner radius $\quad \operatorname{raA}_{2}:=\left[\mathrm{raA1}^{3}-\left(\frac{3 \cdot \delta \mathrm{MaA}_{2}}{4 \cdot \pi \cdot \rho \mathrm{aA}_{2}}\right)\right]^{0.333} \quad \mathrm{raA}_{2}=0.59 \quad \delta \mathrm{raA}_{2}:=\mathrm{raA}-\mathrm{raA}_{2}$ $2^{\text {nd }}$ qtr layer thickness $\quad \delta \mathrm{raA}_{2}=0.017 \quad \mathrm{~cm}$. Radial KE/exhaust energy efficiency $\eta_{\text {ex }}(1.5,1)=0.61$ $2^{\text {nd }}$ qtr drive energy $\quad \operatorname{EdA}_{2}:=0.385 \cdot 10^{6} \cdot \eta_{e x}(1.5,1)^{-1} \quad \operatorname{EdA}_{2}=6.36 \times 10^{5} \quad$ J. Power: $\quad \operatorname{PdA} 2:=\operatorname{EdA}_{2} \cdot\left(\delta \mathrm{tA}_{2}\right)^{-1}$ $\operatorname{PdA}_{2} \cdot 10^{-12}=53 \quad$ TW. 2nd qtr energy density $\quad \mathrm{WdA}_{2}:=\operatorname{EdA}_{2} \cdot\left(\delta \mathrm{MaA}_{2}\right)^{-1} \quad \mathrm{WdA}_{2}=2.77 \times 10^{7} \quad \mathrm{~J} / \mathrm{g}$ $2^{\text {nd }}$ quarter ablation front pressure $\quad \mathrm{WdA}_{2} \cdot \rho \mathrm{aA}_{2} \cdot 10^{6} \cdot 2^{-1} \cdot 10^{-11}=41.5 \quad \mathrm{MB} \quad \mathrm{WdA} \cdot \frac{\mathrm{m}_{\mathrm{h}}}{10 \cdot \mathrm{e}}=29 \mathrm{eV}$ equiv. $2^{\text {nd }}$ qtr shell volume $\delta \mathrm{VaA}_{2}$, is $:=2 \cdot \pi \cdot\left(\mathrm{raA}_{2}+\frac{\delta \mathrm{raA}_{2}}{2}\right)^{2} \cdot \delta \mathrm{raA}_{2} \cdot \sin \left(\theta_{\text {is }}+\frac{\pi}{32}\right) \cdot \frac{\pi}{16} \quad \mathrm{~cm}^{3}$ 2. $\sum_{\text {is }=1}^{8} \delta \mathrm{VaA}_{2}$ is $=0.075 \quad \mathrm{~cm}^{3} \quad \frac{4}{3} \cdot \pi \cdot\left[\left(\mathrm{raA}_{2}+\delta \mathrm{raA}_{2}\right)^{3}-\left(\mathrm{raA}_{2}\right)^{3}\right]=0.075 \quad$ Shell volume checks OK! is $=1$
Beam convergence angle $\quad \theta b_{\text {is }}:=0.125 \cdot \sin \left(\theta_{\text {is }}\right) \quad$ Beam-pathlength-2 Beam range $\quad \rho \mathrm{raA}_{2}$, is $:=\rho \mathrm{aA}_{2} \cdot \delta \mathrm{zaA}_{2}$, is $\quad$ \& energy $\mathbf{E b o 2}_{2}$ is $:=\mathbf{E}_{\mathbf{b f}}\left(\rho \mathrm{AA}_{2}, \rho \operatorname{raA}\right.$, is $\left., 0.01,29, \mathbf{A}_{\mathbf{b}}, \mathbf{A}_{\mathbf{t}}, \mathbf{Z}_{\mathbf{b}}, \mathbf{Z}_{\mathbf{t}}\right)$ Incident beam radius vs polar angle $\theta \quad \mathrm{rbA}_{2}$ is $:=\operatorname{raA}_{2} \cdot \sin \left(\theta_{\text {is }}+\frac{\pi}{32}\right) \quad \operatorname{rbA}_{2}, 9:=\operatorname{raA} 1+\delta z_{2} A_{2}, 9 \cdot \theta b_{9}$ Beam illumination width (cm) per $\theta$ increment $\quad \delta \mathrm{rbA}_{2}$, is $:=\left(\mathrm{raA}_{2}+\frac{\delta \mathrm{raA}_{2}}{2}\right) \cdot \sin \left(\theta_{\text {is }}+\frac{\pi}{16}\right)-\mathrm{raA}_{2} \cdot \sin \left(\theta_{\text {is }}\right)$ Beam deposition intensity $\left(r_{b}\right) \quad \operatorname{IdA}_{2}$, is $:=\operatorname{WdA}_{2} \cdot \delta \operatorname{VaA}_{2}$, is $\cdot \rho \mathrm{aA}_{2} \cdot\left(\delta \mathrm{tA}_{2} \cdot 2 \cdot \pi \cdot \operatorname{rbA} 2 \text {, is } \cdot \delta \mathrm{rbA}_{2}, \text { is }\right)^{-1} \quad \operatorname{IdA}, 9:=0$ $\operatorname{Ebo2,9}:=\operatorname{Ebo2,8}$

Table 16: 2-D polar drive requirements for Case A, $2^{\text {nd }}$ quarter ablation period.

\begin{tabular}{|c|c|c|c|c|c|}
\hline$\theta_{\text {is }}=$ & $\operatorname{rbA}_{2}$, is $=$ & $\delta \mathbf{z a A}_{2}$, is $=$ & $\operatorname{\rho raA}_{2}$, is $=$ & $\operatorname{IdA} A_{2}$ is $\cdot 10^{-12}=$ & Ebo2, is $\cdot 10^{-6}=$ \\
\hline 0 & 0.06 & 0.02 & 0.005 & 12 & 247 \\
\hline 0.2 & 0.17 & 0.02 & 0.005 & 12 & 250 \\
\hline 0.39 & 0.28 & 0.02 & 0.0053 & 13 & 258 \\
\hline 0.59 & 0.38 & 0.02 & 0.0057 & 14 & 273 \\
\hline 0.79 & 0.46 & 0.02 & 0.0065 & 17 & 298 \\
\hline 0.98 & 0.52 & 0.03 & 0.0078 & 22 & 338 \\
\hline 1.18 & 0.57 & 0.03 & 0.0102 & 33 & 406 \\
\hline 1.37 & 0.59 & 0.05 & 0.0158 & 70 & 541 \\
\hline 1.57 & 0.63 & 0.13 & 0.0398 & 0 & 541 \\
\hline $\begin{array}{l}\text { Polar } \\
\text { angle (rad) }\end{array}$ & $\begin{array}{l}\text { Beam } \\
\text { radius }(\mathrm{cm})\end{array}$ & $\begin{array}{l}\text { Shell } \\
z \text { depth }(\mathrm{cm})\end{array}$ & $\begin{array}{l}\text { Beam } \\
\text { range } \mathrm{g} / \mathrm{cm}^{2}\end{array}$ & $\begin{array}{l}\text { Beam } \\
\text { Intensity } \mathrm{TW} / \mathrm{cm}^{2}\end{array}$ & $\begin{array}{l}\text { Argon Beam Energy } \\
\text { (MeV) (No parasitic } \\
\text { ablation plasma loss) }\end{array}$ \\
\hline
\end{tabular}


Now lets correct for ablation plasma in this ablation period of The second quarter ablated plasma mass expands in this time interval to a radius $u_{\text {ave } 2}:=0.7 \cdot 10^{7}$ $\operatorname{rpA}_{2}:=\operatorname{raA} 1+\left(u_{e x}(1.5,1)-u_{a v e 2}\right) \cdot \delta t_{2} \quad \operatorname{rpA}_{2}=0.7 \quad \mathbf{c m}$

and to a mass density ${\rho \mathrm{pA}_{2}}_{2}:=\delta \mathrm{MaA}_{2} \cdot\left[\frac{4}{3} \cdot \pi \cdot\left[\left(\operatorname{rpA}_{2}\right)^{3}-\operatorname{raA1}^{3}\right]\right]^{-1}$

$$
\rho \mathrm{pA}_{2}=\mathbf{0 . 0 2 9} \quad \mathrm{g} / \mathrm{cm} 3
$$

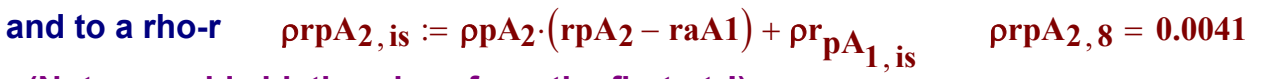
(Note we add ablation rho-r from the first qtr!)

$\frac{\delta \mathrm{tA}_{2}}{10^{-9}}=11.95 \quad \mathrm{~ns}$

Lets make a first order correction to the exhaust plasma temperature, assuming input goes both to hydro

$$
\Delta \operatorname{EbpA}_{2}, \text { is }:=\left(\mathbf{E}_{\mathbf{b o}}\left(\rho \operatorname{\rho p} \mathbf{A}_{2}, \text { is }, \rho r a A_{2}, \text { is }, \rho p A_{2}, \rho A_{2}, 190,29, \mathbf{A}_{\mathbf{b}}, \mathbf{A}_{\mathbf{t}}, \mathbf{Z}_{\mathbf{b}}, \mathbf{Z}_{\mathbf{t}}\right)-\mathbf{E b o 2 , \text { is }}\right) \cdot(\mathbf{E b o 2}, \text { is })^{-1}
$$

and into incremental thermal energy $3 \Delta T_{p}$, and for the moment lets assume the ablated plasma density does not change; then we have, adding the last qtr beam input to heating on top:

$$
\begin{aligned}
& \operatorname{TpA}_{2} \text {, is }:=\frac{\operatorname{IdA}_{2}, \text { is } \cdot \delta \mathrm{tA}_{2} \cdot \Delta \operatorname{EbpA_{2},\text {is}}}{\rho r p A_{2}, \text { is }} \cdot \frac{m_{h}}{6 \cdot \mathrm{e}} \ldots \\
& +\operatorname{TpA}_{1}, \text { is } \\
& T_{\mathrm{ex}}(1.5,1)=26.3
\end{aligned}
$$

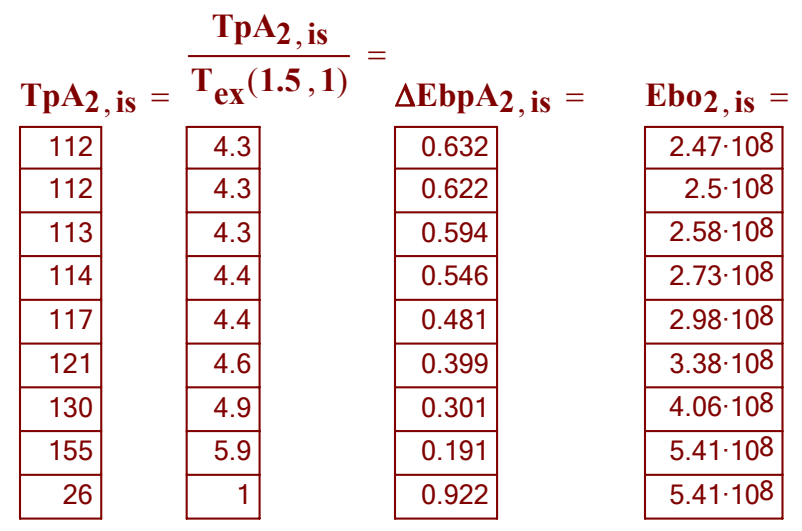

One can see in this table that the ablation plasma is substantially heated (Te increases 10X)

\begin{tabular}{|c|c|c|}
\hline $1.17 \cdot 107$ & 1.19 & 0.24 \\
\hline $1.17 \cdot 107$ & 1.22 & 0.24 \\
\hline $1.18 \cdot 107$ & 1.31 & 0.24 \\
\hline $1.19 \cdot 107$ & 1.48 & 0.24 \\
\hline $1.2 \cdot 107$ & 1.78 & 0.24 \\
\hline $1.23 \cdot 107$ & 2.36 & 0.25 \\
\hline $1.29 \cdot 107$ & 3.67 & 0.26 \\
\hline $1.44 \cdot 107$ & 8.71 & 0.29 \\
\hline 0 & 0 & 0 \\
\hline
\end{tabular}
(more in the 2 nd qtr of the pulse). Check if heating (pressurization) can still cause significant enhanced expansion in the shorter transverse to the beam channel (polar axis) direction in the shorter time $\delta$ t $A_{2}$ :

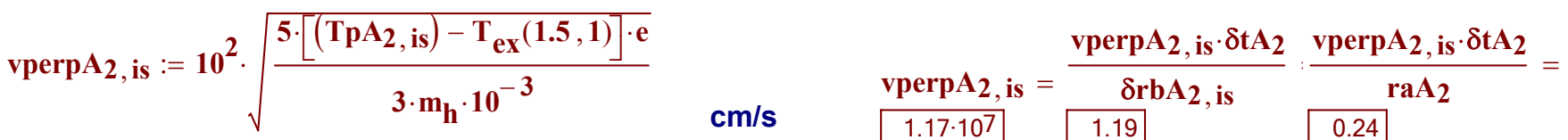

$$
\begin{aligned}
& \rho_{\mathbf{p A}_{2, \text { is }}}:=\rho p \mathbf{A}_{2} \cdot\left(\frac{\operatorname{TpA}_{2,1}}{\operatorname{TpA_{2},\text {is}}}\right) \cdot\left(1+\frac{\operatorname{vperpA_{2},\text {is}} \cdot \delta \mathbf{t} \mathbf{A}_{2}}{\operatorname{raA_{2}}}\right)^{-2} \\
& \text { and } \quad \rho_{\mathbf{p A}_{2,9}}:=\rho \mathbf{p} \mathbf{A}_{2} \\
& \rho \mathbf{p}_{2, \text { is }}:=\operatorname{\rho rpA_{2},\text {is}} \cdot\left(\frac{\operatorname{Tp} A_{2,1}}{\operatorname{Tp} A_{2}, \text { is }}\right) \cdot\left(1+\frac{\operatorname{vperpA_{2},\text {is}} \cdot \delta t A_{2}}{\operatorname{raA} A_{2}}\right)^{-2} \\
& \rho \mathbf{p}_{\mathbf{2}, 9}:=\operatorname{\rho rpA_{2},9}
\end{aligned}
$$

The corrected incident beam energy requirement adjusted for heated ablation plasma loss is

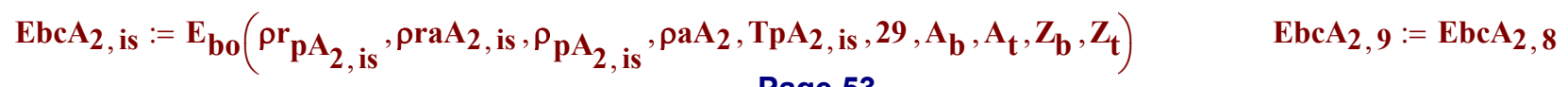

\section{Page 53}




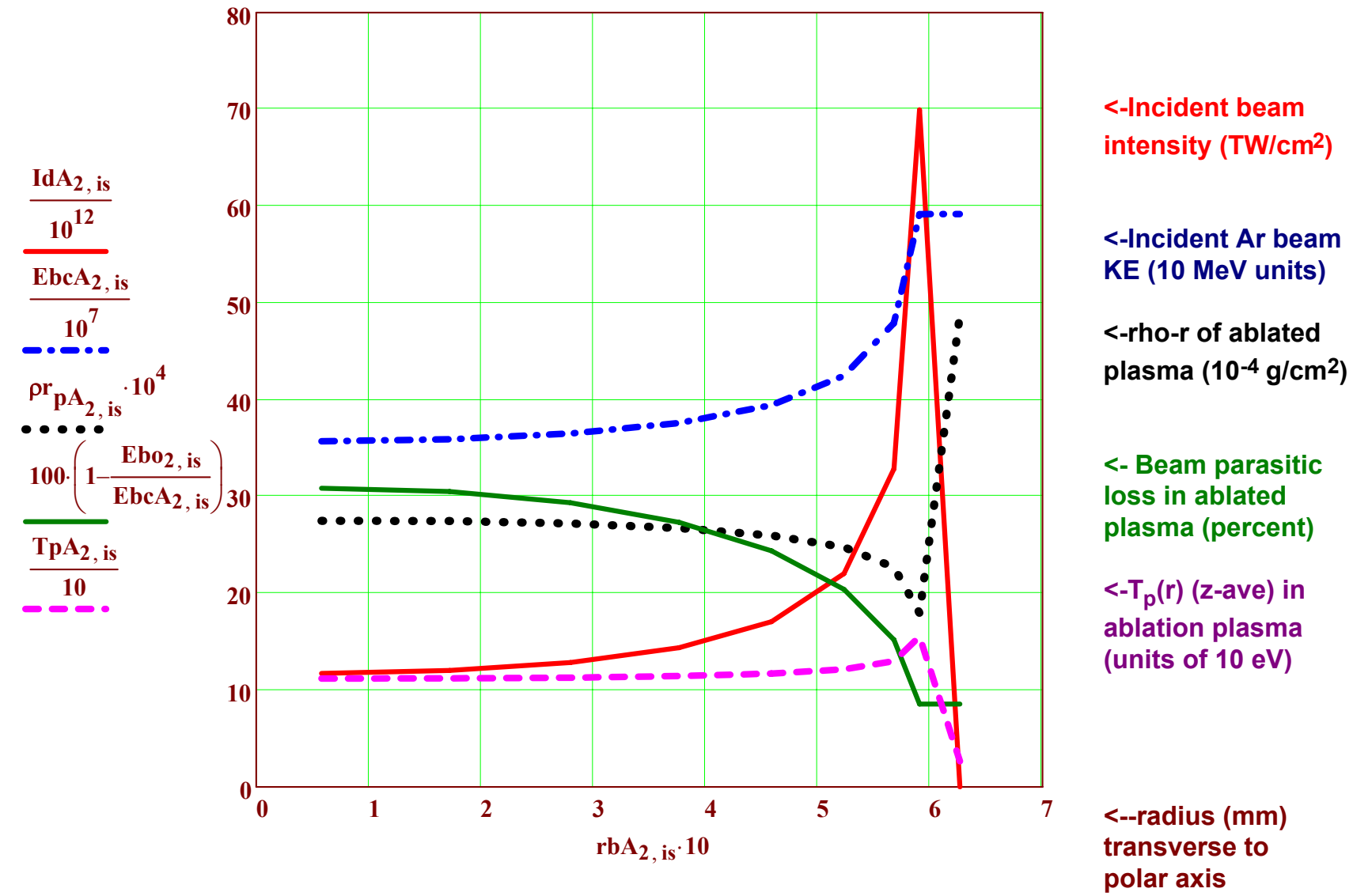

Figure 36: Plots of polar beam drive intensity (TW/ $\mathrm{cm}^{2}$, one of two sides), incident Ar beam energy (in $10 \mathrm{MeV}$ units), rho-r of ablated plasma column density $\left(10^{-4} \mathrm{~g} / \mathrm{cm}^{2}\right.$ units) (dotted black line), percent beam loss in ablated plasma, and the temperature $T_{p}$ of the ablation plasma (in $10 \mathrm{eV}$ units), as functions of radius in the beam channel, transverse to the polar axis near the target, during the second quarter of the ablation drive pulse for the large T-lean target case $A$. Note beam intensity peak is now $\sim 6 X$ intensity on axis, and percent beam loss on ablated plasma is higher. Also, note the local ablation temperature $\left(T_{p}\left(r_{b}\right)\right.$ has increased with the beam intensity, and a greater beam heating effect digging a hole in the density at the beam rim position, compared to the 1 st quarter ablation period.

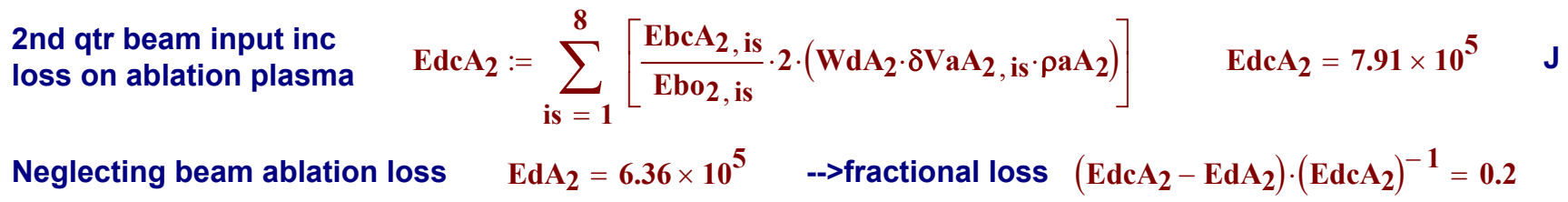


Polar drive parameters for Case $\mathrm{A}, 3 \underline{\text { rd }} \underline{\text { quarter of ablation drive }} \quad \mathrm{tA}_{3}:=\delta \mathrm{tA}_{1}+\delta \mathrm{tA}_{2}$

$3^{\text {rd }}$ quarter ablator mass $\quad \delta \mathrm{MaA}_{3}=0.023 \quad \mathrm{~g} \quad \mathrm{tA}_{3}=9.295 \times 10^{-8} \mathrm{~s} \quad @$ density $\quad \rho \mathrm{aA}_{3}:=5 \cdot \rho_{\mathrm{Ho}}$

$3^{\text {rd }}$ qtr outer radius $\quad \mathrm{raA2}:=0.54 \quad \mathrm{~cm}, \delta \mathrm{MaA}_{3}$ ablated over time interval $\quad \delta$ tA3 $:=6 \cdot 10^{-9} \mathrm{~s}$

3rd qtr inner radius $\quad \mathrm{raA}_{3}:=\left[\mathrm{raA}^{3}-\left(\frac{3 \cdot \delta \mathrm{MaA}_{3}}{4 \cdot \pi \cdot \rho \mathrm{aA}_{3}}\right)\right]^{0.333} \quad \mathrm{raA}_{3}=0.53 \quad \delta \mathrm{raA}_{3}:=\mathrm{raA}_{2}-\mathrm{raA}_{3}$

$3^{\text {rd }}$ qtr layer thickness $\quad \delta \mathrm{raA}_{3}=0.013 \quad \mathrm{~cm}$. Radial KE/exhaust energy efficiency $\eta_{\text {ex }}(1.5,1)=0.61$

$3^{\text {rd }}$ qtr drive energy $\quad \operatorname{EdA}_{3}:=0.385 \cdot 10^{6} \cdot \eta_{\mathrm{ex}}(1.5,1)^{-1} \quad \operatorname{EdA}_{3}=6.36 \times 10^{5} \quad$ J. Power: $\quad \operatorname{PdA} 3:=\operatorname{EdA} 3 \cdot\left(\delta \mathrm{tA}_{3}\right)^{-1}$

$\mathbf{P d A}_{3} \cdot 10^{-12}=106 \quad$ TW. $3^{\text {rd }}$ qtr energy density $\quad \mathrm{WdA}_{3}:=\mathrm{EdA}_{3} \cdot\left(\delta \mathrm{MaA}_{3}\right)^{-1} \quad \mathrm{WdA}_{3}=2.77 \times 10^{7} \quad \mathrm{~J} / \mathrm{g}$

3rd quarter ablation front pressure $\quad \mathrm{WdA}_{3} \cdot \rho \mathrm{aA}_{3} \cdot 10^{6} \cdot 2^{-1} \cdot 10^{-11}=69 \quad \mathrm{MB} \quad \mathrm{WdA} \cdot \frac{\mathrm{m}_{\mathrm{h}}}{10 \cdot \mathrm{e}}=29 \quad \mathrm{eV}$

equiv.

$3^{\text {rd }}$ qtr shell volume $\quad \delta \mathrm{VaA}_{3}$, is $:=2 \cdot \pi \cdot\left(\operatorname{raA}_{3}+\frac{\delta \mathrm{raA}_{3}}{2}\right)^{2} \cdot \delta \mathrm{raA}_{3} \cdot \sin \left(\theta_{\text {is }}+\frac{\pi}{32}\right) \cdot \frac{\pi}{16} \quad \mathrm{~cm}^{3}$

2. $\sum_{\text {is }=1}^{8} \delta \mathrm{VaA}_{3}$, is $=0.045 \quad \mathrm{~cm}^{3} \quad \frac{4}{3} \cdot \pi \cdot\left[\left(\mathrm{raA}_{3}+\delta \mathrm{raA}_{3}\right)^{3}-\left(\mathrm{raA}_{3}\right)^{3}\right]=0.045 \quad$ Shell volume checks OK!

Beam convergence angle $\quad \theta b_{\text {is }}:=\mathbf{0 . 1 2 5} \cdot \sin \left(\theta_{\text {is }}\right) \quad$ Beam-pathlength-3rd qtr $\quad \delta z^{2} A_{3}$, is $:=\frac{\delta \mathrm{raA}_{3}}{\cos \left(\theta_{\text {is }}-\theta b_{\text {is }}\right)}$

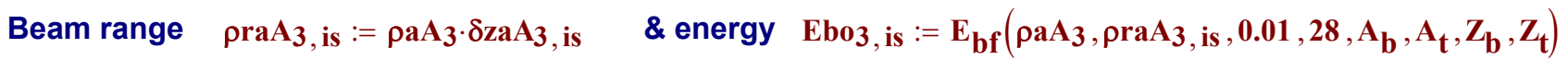

Incident beam radius vs polar angle $\theta \quad \mathrm{rbA}_{3}$, is $:=\operatorname{raA}_{3} \cdot \sin \left(\theta_{\mathrm{is}}+\frac{\pi}{32}\right) \quad \quad \mathrm{rbA}_{3}, 9:=\operatorname{raA} 2+\delta \mathrm{zaA}_{3}, 9 \cdot \theta \mathrm{bb}_{9}$

Beam illumination width (cm) per $\theta$ increment $\quad \delta \mathrm{rbA}_{3}$, is : $=\left(\mathrm{raA}_{3}+\frac{\delta \mathrm{raA}_{3}}{2}\right) \cdot \sin \left(\theta_{\text {is }}+\frac{\pi}{16}\right)-\mathrm{raA}_{3} \cdot \sin \left(\theta_{\text {is }}\right)$

Beam deposition intensity $\left(r_{b}\right) \quad \operatorname{IdA}_{3}$, is $:=\mathrm{WdA}_{3} \cdot \delta \mathrm{VaA}_{3}$, is $\cdot \rho \mathrm{AA}_{3} \cdot\left(\delta \mathrm{tA}_{3} \cdot 2 \cdot \pi \cdot \operatorname{rbA} 3 \text {, is } \cdot \delta \mathrm{rbA}_{3}, \text { is }\right)^{-1} \quad \operatorname{IdA} 3,9:=0$

$\operatorname{Ebo3,9}:=\operatorname{Ebo3}, 8$

Table 17: 2-D polar drive requirements for Case A, $3^{\text {rd }}$ quarter ablation period.

\begin{tabular}{|c|c|c|c|c|c|}
\hline$\theta_{\text {is }}=$ & $\operatorname{rbA}_{3}$, is $=$ & $\delta \mathrm{zaA}_{3}$, is $=$ & $\operatorname{praA}_{3}$, is $=$ & $\operatorname{IdA} 3$, is $\cdot 10^{-12}=$ & Ebo3, is $\cdot 10^{-6}=$ \\
\hline 0 & 0.05 & 0.013 & 0.0063 & 29 & 281 \\
\hline 0.2 & 0.15 & 0.013 & 0.0064 & 30 & 284 \\
\hline 0.39 & 0.25 & 0.013 & 0.0067 & 32 & 294 \\
\hline 0.59 & 0.33 & 0.014 & 0.0072 & 36 & 311 \\
\hline 0.79 & 0.41 & 0.016 & 0.0082 & 43 & 339 \\
\hline 0.98 & 0.47 & 0.02 & 0.0098 & 56 & 384 \\
\hline 1.18 & 0.5 & 0.026 & 0.0129 & 85 & 460 \\
\hline 1.37 & 0.52 & 0.04 & 0.02 & 187 & 610 \\
\hline 1.57 & 0.55 & 0.1 & 0.0502 & 0 & 610 \\
\hline $\begin{array}{l}\text { Polar } \\
\text { angle (rad) }\end{array}$ & $\begin{array}{l}\text { Beam } \\
\text { radius }(\mathrm{cm})\end{array}$ & $\begin{array}{l}\text { Shell } \\
z \text { depth }(\mathbf{c m})\end{array}$ & $\begin{array}{l}\text { Beam } \\
\text { range } \mathrm{g} / \mathrm{cm}^{2}\end{array}$ & $\begin{array}{l}\text { Beam } \\
\text { Intensity } \mathrm{TW} / \mathrm{cm}^{2}\end{array}$ & $\begin{array}{l}\text { Argon Beam Energy } \\
\text { (MeV) (Neglect parasitic } \\
\text { ablation plasma loss) }\end{array}$ \\
\hline
\end{tabular}

Page 55 
Now lets correct for ablation plasma in this ablation period of The third quarter ablated plasma mass expands in this time interval to a radius

$$
\operatorname{rpA}_{3}:=\operatorname{raA} 2+\left(u_{e x}(1.5,1)-u_{\text {ave3 }}\right) \cdot \delta \mathbf{t A}_{3}
$$

$u_{\text {ave3 }}:=1.3 \cdot 10^{7} \quad \operatorname{rpA}_{3}:=\operatorname{raA} 2+\left(u_{e x}(1.5,1)-u_{\text {ave3 }}\right) \cdot \delta \mathrm{tA}_{3} \quad \operatorname{rpA}_{3}=0.6 \quad \mathrm{~cm}$

$$
\frac{\delta \mathrm{tA}_{3}}{10^{-9}}=6
$$

and to a mass density $\rho \mathrm{pA}_{3}:=\delta \mathrm{MaA}_{3} \cdot\left[\frac{4}{3} \cdot \pi \cdot\left[\left(\mathrm{rpA}_{3}\right)^{3}-\mathrm{raA}_{2}\right]^{-1} \quad \rho\right]_{3}=0.185 \quad \mathrm{~g} / \mathrm{cm} 3$

and to a rho-r $\quad \rho r p A_{3}$, is $:=\rho p A_{3} \cdot\left(\operatorname{rp} A_{3}-\operatorname{raA} 2\right)+\rho r_{p} A_{2, \text { is }} \quad \rho r p A_{3}, 8=0.0077$

(Note we add in ablation rho-r from the $2^{\text {nd }}$ qtr!)

$\mathrm{g} / \mathrm{cm}^{2}$, now $\sim 95 \%$ of the ave $3^{\text {rd }}$ qtr ablator range.

Lets make a first order correction to the exhaust plasma temperature, assuming all beam energy deposited into the ablation plasma mass within the beam channel

$$
\Delta \mathbf{E b p A}_{3}, \text { is }:=\left(\mathbf{E}_{\mathbf{b o}}\left(\operatorname{\rho rp} \mathbf{A}_{3}, \text { is }, \rho \operatorname{\rho ra} \mathbf{A}_{3}, \text { is }, \rho p \mathbf{A}_{3}, \rho \mathbf{A A}_{3}, 300,29, \mathbf{A}_{\mathbf{b}}, \mathbf{A}_{\mathbf{t}}, \mathbf{Z}_{\mathbf{b}}, \mathbf{Z}_{\mathbf{t}}\right)-\mathbf{E b o 3}, \text { is }\right) \cdot(\mathbf{E b o 3}, \text { is })^{-1}
$$

goes into incremental thermal energy $3 \Delta T_{p}$, and for the moment lets assume the ablated plasma density does not change; then we have, adding the last qtr beam input to heating on top:

$$
\begin{aligned}
& \operatorname{TpA}_{3}, \text { is }:=\frac{\operatorname{IdA}_{3}, \text { is } \cdot \delta t A_{3} \cdot \Delta \operatorname{Ebp} A_{3}, \text { is }}{\rho r p A_{3}, \text { is }} \cdot \frac{m_{h}}{6 \cdot e} \ldots \\
& +\mathbf{T p A} \mathbf{A}_{2} \text {, is } \\
& T_{\text {ex }}(1.5,1)=26.3
\end{aligned}
$$

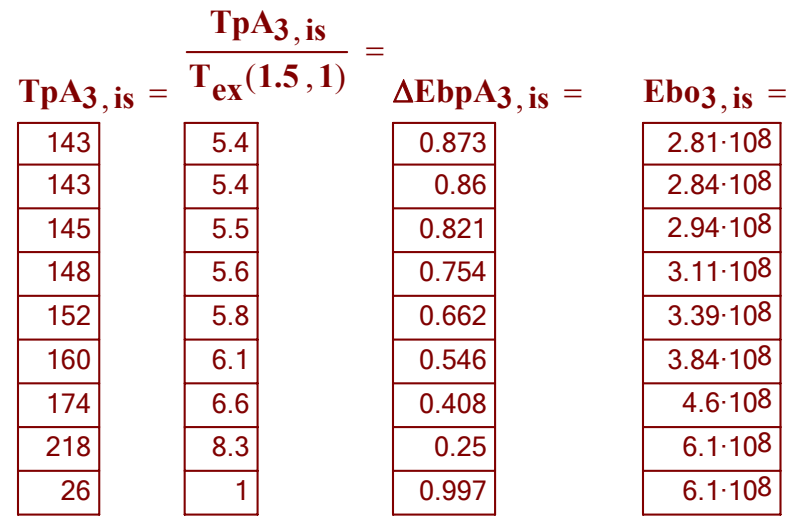

One can see in this table that the ablation plasma is substantially heated (Te increases 10X) (more in the 3rd qtr of the pulse). Check if heating (pressurization) can still cause significant enhanced expansion in the shorter transverse to the beam channel (polar axis) direction in the shorter time $\delta$ t $A_{3}$ :

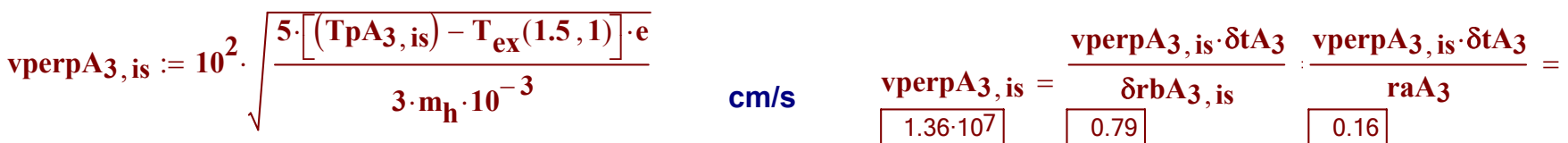

$$
\begin{aligned}
& \rho_{\mathbf{p A}_{3, \text { is }}}:=\rho \mathbf{p} \mathbf{A}_{3} \cdot\left(\frac{\operatorname{TpA}_{3}, \mathbf{1}}{\operatorname{Tp} \mathbf{A}_{3, \text { is }}}\right) \cdot\left(1+\frac{\operatorname{vperpA_{3},\text {is}} \cdot \delta \mathbf{t} \mathbf{A}_{3}}{\operatorname{raA}_{3}}\right)^{-2} \\
& \text { and } \quad \rho_{\mathbf{p A}_{3,9}}:=\rho \mathbf{p A _ { 3 }} \\
& \rho \mathbf{p A}_{3, \text { is }}:=\operatorname{\rho rpA_{3},\text {is}} \cdot\left(\frac{\operatorname{Tp} A_{3}, 1}{\operatorname{Tp} A_{3}, \text { is }}\right) \cdot\left(1+\frac{\operatorname{vperp} A_{3}, \text { is } \cdot \delta A_{3}}{\operatorname{raA} A_{3}}\right)^{-2} \\
& \rho \mathbf{p A}_{3,9}:=\rho \operatorname{prpA_{3},9}
\end{aligned}
$$

\begin{tabular}{|r|}
\hline 0.79 \\
\hline 0.81 \\
\hline 0.87 \\
\hline 0.99 \\
\hline 1.2 \\
\hline 1.6 \\
\hline 2.55 \\
\hline 6.41 \\
\hline 0 \\
\hline
\end{tabular}

\begin{tabular}{|r|}
\hline 0.16 \\
\hline 0.16 \\
\hline 0.16 \\
\hline 0.16 \\
\hline 0.16 \\
\hline 0.17 \\
\hline 0.17 \\
\hline 0.2 \\
\hline 0 \\
\hline
\end{tabular}

The corrected incident beam energy requirement adjusted for heated ablation plasma loss is

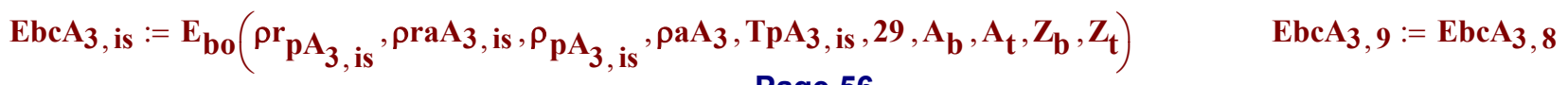




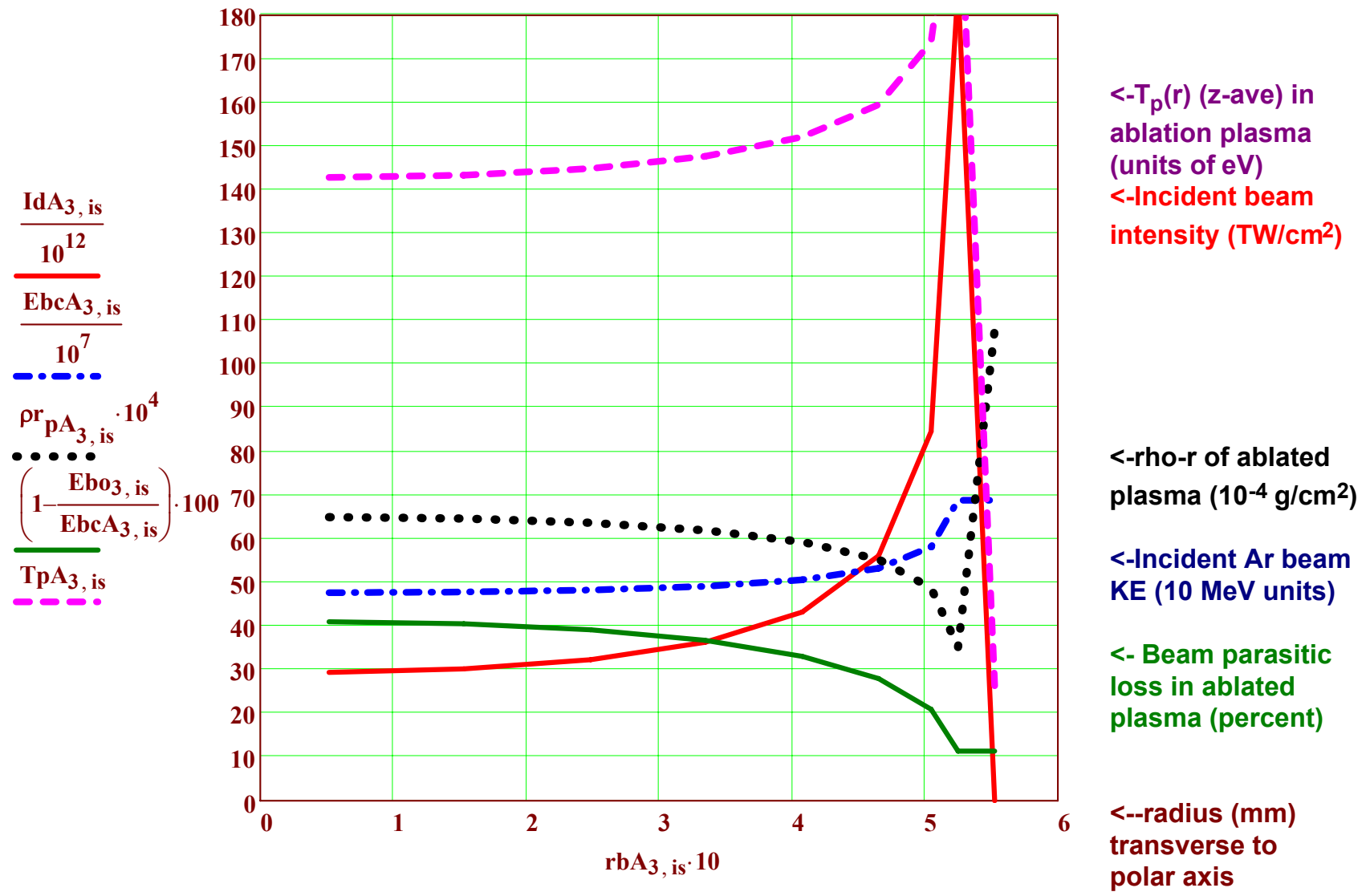

Figure 37: Plots of polar beam drive intensity (TW/ $\mathrm{cm}^{2}$, one of two sides), incident Ar beam energy (in $10 \mathrm{MeV}$ units), rho-r of ablated plasma column density $\left(10^{-4} \mathrm{~g} / \mathrm{cm}^{2}\right.$ units) (dotted black line), percent beam loss in ablated plasma, and the temperature $T_{p}$ of the ablation plasma (in eV units), as functions of radius in the beam channel, transverse to the polar axis near the target, during the third quarter of the ablation drive pulse for the large T-lean target case A. Note the ablation cloud rho-r is now comparable to the 3rd quarter shell rho-r, essentially doubling the required ion range on axis. However, the beam losses on ablation plasma over the whole profile, while larger than in the 2nd quarter, are greatly mitigated compared to what they would have been without taking hole-boring into account, especially because of 2-D polar drive and beam heating effects included.

\begin{tabular}{|c|c|c|c|c|}
\hline $\begin{array}{l}\text { 3rd qtr beam input inc } \\
\text { loss on ablation plasma }\end{array}$ & $:=$ & $\frac{\text { EbcA3, is }}{\text { Ebo3, is }}$ & $\cdot\left(\mathrm{WdAA}_{3} \cdot \delta \mathrm{VaA}_{3}\right.$, is $\left.\cdot \rho \mathrm{aA}_{3}\right)$ & $\mathrm{EdcA}_{3}=8.69 \times 10^{5}$ \\
\hline Neglecting beam ablation loss & EdA & $.36 \times 10^{5}$ & -->fractional loss $(E$ & $\left.3-\operatorname{EdA}_{3}\right) \cdot\left(\operatorname{EdcA}_{3}\right)^{-1}=0.27$ \\
\hline
\end{tabular}




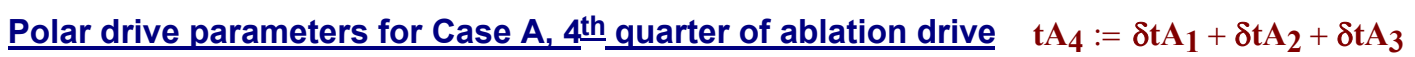

$4^{\text {th }}$ quarter ablator mass $\quad \delta \mathrm{MaA}_{4}=0.023 \quad \mathrm{~g} \quad \mathrm{tA}_{4}=9.9 \times 10^{-8} \quad \mathrm{~s}, @$ density $\quad \rho_{4}:=12 \cdot \rho_{\mathrm{Ho}}$

$4^{\text {th }}$ qtr outer radius $\quad \operatorname{raA3}:=0.445 \quad \mathrm{~cm}$, ablated over time interval $\delta \mathrm{tA}_{4}:=6 \cdot 10^{-9} \mathrm{~s}$

$4^{\text {th }}$ qtr inner radius $\quad \operatorname{raA}_{4}:=\left[\operatorname{raA}^{3}-\left(\frac{3 \cdot \delta \mathrm{MaA}_{4}}{4 \cdot \pi \cdot \rho \mathrm{AA}_{4}}\right)\right]^{0.333} \quad \operatorname{raA}_{4}=0.44 \quad \delta \mathrm{raA}_{4}:=\operatorname{raA}-\mathrm{raA}_{4}$

$4^{\text {th }}$ qtr layer thickness $\quad \delta \mathrm{raA}_{4}=0.007 \quad \mathrm{~cm}$. Radial KE/exhaust energy efficiency $\eta_{\mathrm{ex}}(1.5,1)=0.61$

$4^{\text {th }}$ qtr drive energy $\quad \operatorname{EdA4}_{4}:=0.385 \cdot 10^{6} \cdot \eta_{\mathrm{ex}}(1.5,1)^{-1} \quad \operatorname{EdA}_{4}=6.36 \times 10^{5} \quad \mathrm{~J} . \operatorname{Power}^{*}: \operatorname{PdA}_{4}:=\operatorname{EdA4} \cdot\left(\delta \mathrm{tA}_{4}\right)^{-1}$

$\operatorname{PdA}_{4} \cdot 10^{-12}=106 \quad$ TW. $4^{\text {th }}$ qtr energy density $\quad \mathrm{WdA}_{4}:=\operatorname{EdA4} \cdot\left(\delta \mathrm{MaA}_{4}\right)^{-1} \quad \mathrm{WdA}_{4}=2.77 \times 10^{7} \quad \mathrm{~J} / \mathrm{g}$

$4^{\text {th }}$ quarter ablation front pressure $\quad \mathrm{WdA}_{4} \cdot \rho \mathrm{AA}_{4} \cdot 10^{6} \cdot 3^{-1} \cdot 10^{-11}=111 \quad \mathrm{MB} \quad \mathrm{WdA4} \cdot \frac{\mathrm{m}_{\mathrm{h}}}{10 \cdot \mathrm{e}}=29 \quad \mathrm{eV}$

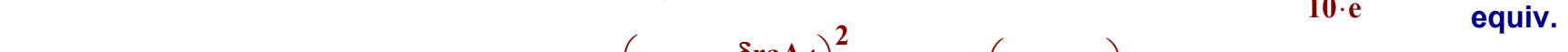

$4^{\text {th }}$ qtr shell volume

$\delta \mathrm{VaA}_{4}$, is $:=2 \cdot \pi \cdot\left(\operatorname{raA}_{4}+\frac{\delta \mathrm{raA}_{4}}{2}\right)^{2} \cdot \delta \mathrm{raA}_{4} \cdot \sin \left(\theta_{\mathrm{is}}+\frac{\pi}{32}\right) \cdot \frac{\pi}{16} \quad \mathrm{~cm}^{3}$

2. $\sum_{\text {is }=1}^{8} \delta \mathrm{VaA}_{4}$, is $=0.018 \quad \mathrm{~cm}^{3} \quad \frac{4}{3} \cdot \pi \cdot\left[\left(\mathrm{raA}_{4}+\delta \mathrm{raA}_{4}\right)^{3}-\left(\mathrm{raA}_{4}\right)^{3}\right]=0.018 \quad$ Shell volume checks OK!

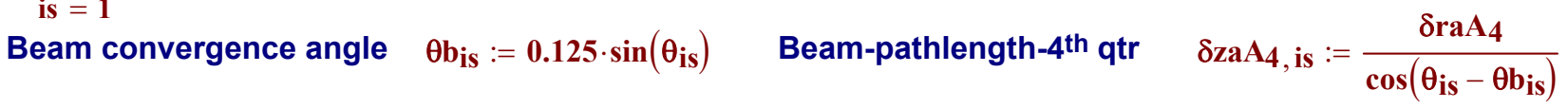

Beam range $\rho \mathrm{AA}_{4}$, is $:=\rho \mathrm{aA}_{4} \cdot \delta \mathrm{zaA}_{4}$, is $\quad$ \& energy* $\quad \mathbf{E b o 4}_{4}$ is $:=\mathbf{E}_{\mathbf{b f}}\left(\rho \mathbf{a A}, \rho \mathrm{ArA}_{4}\right.$, is $\left., 0.01,29, \mathbf{A}_{\mathbf{b}}, \mathbf{A}_{\mathbf{t}}, \mathbf{Z}_{\mathbf{b}}, \mathbf{Z}_{\mathbf{t}}\right)$ Incident beam radius vs polar angle $\theta \quad \operatorname{rbA}_{4}$, is $:=\operatorname{raA}_{4} \cdot \sin \left(\theta_{\text {is }}+\frac{\pi}{32}\right) \quad \quad \operatorname{rbA} 4,9:=\operatorname{raA} 3+\delta z a A_{4}, 9 \cdot \theta b 9$ Beam illumination width (cm) per $\theta$ increment $\quad \delta \mathbf{r b A}_{4}$, is $:=\left(\operatorname{raA}_{4}+\frac{\delta \mathbf{r a A}_{4}}{2}\right) \cdot \sin \left(\theta_{\text {is }}+\frac{\pi}{16}\right)-\operatorname{raA} 4 \cdot \sin \left(\theta_{\text {is }}\right)$

Beam deposition intensity $\left(r_{b}\right) \quad \operatorname{IdA}_{4}$, is $:=\mathrm{WdA}_{4} \cdot \delta \mathrm{VaA}_{4}$, is $\cdot \rho \mathrm{AA}_{4} \cdot\left(\delta \mathrm{tA}_{4} \cdot 2 \cdot \pi \cdot \operatorname{rbA} 4, \text { is } \cdot \delta \mathrm{rbA}_{4}, \text { is }\right)^{-1} \quad \operatorname{IdA}_{4}, 9:=0$ *Beam power, energy before taking beam losses into account

$\operatorname{Ebo4,9}:=\operatorname{Ebo4,8}$

Table 18: 2-D polar drive requirements for Case A, $4^{\text {th }}$ quarter ablation period.

\begin{tabular}{|c|c|c|c|c|c|}
\hline$\theta_{\mathbf{i s}}=$ & $\operatorname{rbA}_{4}$, is $=$ & $\delta \operatorname{zaAA}_{4}$, is $=$ & $\operatorname{\rho raA}_{4}$, is $=$ & $\operatorname{IdA} 4$, is $\cdot 10^{-12}=$ & Ebo4, is $\cdot 10^{-6}=$ \\
\hline 0 & 0.04 & 0.01 & 0.009 & 42 & 341 \\
\hline 0.2 & 0.13 & 0.01 & 0.0091 & 43 & 344 \\
\hline 0.39 & 0.21 & 0.01 & 0.0095 & 47 & 355 \\
\hline 0.59 & 0.28 & 0.01 & 0.0103 & 52 & 376 \\
\hline 0.79 & 0.34 & 0.01 & 0.0117 & 63 & 409 \\
\hline 0.98 & 0.39 & 0.01 & 0.014 & 82 & 462 \\
\hline 1.18 & 0.42 & 0.02 & 0.0184 & 127 & 552 \\
\hline 1.37 & 0.44 & 0.02 & 0.0286 & 298 & 730 \\
\hline 1.57 & 0.45 & 0.06 & 0.072 & 0 & 730 \\
\hline $\begin{array}{l}\text { Polar } \\
\text { angle (rad) }\end{array}$ & $\begin{array}{l}\text { Beam } \\
\text { radius }(\mathbf{c m})\end{array}$ & $\begin{array}{l}\text { Shell } \\
\text { z depth }(\mathrm{cm})\end{array}$ & $\begin{array}{l}\text { Beam } \\
\text { range } \mathrm{g} / \mathrm{cm}^{2}\end{array}$ & $\begin{array}{l}\text { Beam } \\
\text { Intensity } \mathrm{TW} / \mathrm{cm}^{2}\end{array}$ & $\begin{array}{l}\text { Argon Beam Energy } \\
\text { (MeV) (No parasitic } \\
\text { ablation plasma loss) }\end{array}$ \\
\hline
\end{tabular}

Page 58 
Now lets correct for ablation plasma in this ablation period of The fourth quarter ablated plasma mass expands in this time interval to a radius

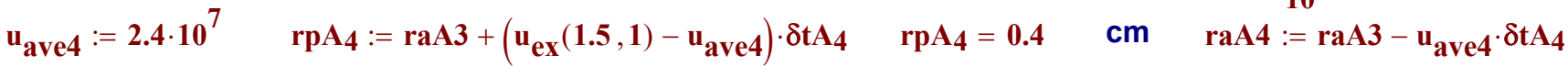
and to a mass density $\rho \mathrm{pA}_{4}:=\delta \mathrm{MaA}_{4} \cdot\left[\frac{4}{3} \cdot \pi \cdot\left[\left(\operatorname{rpA}_{4}\right)^{3}-\mathrm{raA}_{4}^{3}\right]\right]^{-1} \quad \rho \mathrm{pA}_{4}=0.13 \quad \mathrm{~g} / \mathrm{cm} 3$ and to a rho-r $\quad \rho r p A_{4}$, is $:=\rho \mathrm{pA}_{4} \cdot\left(\operatorname{rpA}_{4}-\mathrm{raA}_{4}\right)+\rho \mathrm{pA}_{3}$, is $\quad \rho r p A_{4}, 8=0.018 \quad \mathrm{~g} / \mathrm{cm}^{2}$, now $\sim$ equal to the (Note we add ablation rho-r's accumulated up through the third qtr!) ave $4^{\text {th }}$ qtr ablator range.

Lets make a first order correction to the exhaust plasma temperature, assuming all beam energy deposited into the ablation plasma mass within the beam channel

$$
\Delta \mathbf{E b p A}_{4}, \text { is }:=\left(\mathbf{E}_{\mathbf{b o}}\left(\rho \operatorname{\rho rp} \mathbf{A}_{4}, \text { is }, \rho \operatorname{\rho raA} 4, \text { is }, \rho p A_{4}, \rho \mathbf{A A}_{4}, 250,29, \mathbf{A}_{\mathbf{b}}, \mathbf{A}_{\mathbf{t}}, \mathbf{Z}_{\mathbf{b}}, \mathbf{Z}_{\mathbf{t}}\right)-\mathbf{E b o 4}_{4} \text { is }\right) \cdot\left(\mathbf{E b o 4}_{\text {, is }}\right)^{-1}
$$

goes into incremental thermal energy $3 \Delta T_{p}$, and for the moment lets assume the ablated plasma density does not change; then we have, adding the last qtr beam input to heating on top :

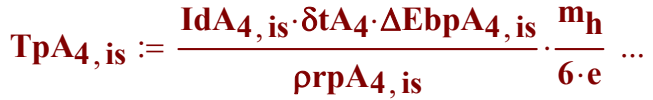

$$
\begin{aligned}
& +\operatorname{TpA}_{3} \text {, is } \\
& T_{\text {ex }}(1.5,1)=26.3
\end{aligned}
$$

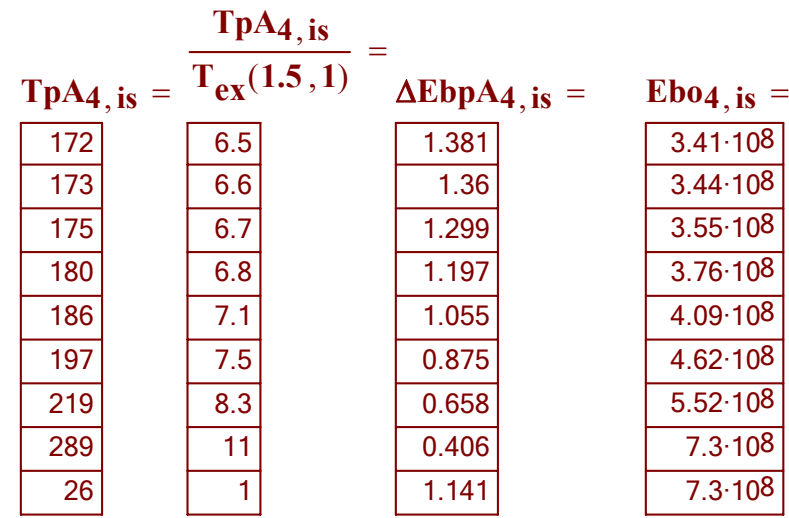

One can see in this table that the ablation plasma is substantially heated (Te increases 10-17X) (most in this 4th qtr of the pulse). Check if heating (pressurization) can still cause significant enhanced expansion in the shorter transverse to the beam channel (polar axis) direction in the shorter time $\delta$ tA $\mathrm{A}_{4}$

\begin{tabular}{|c|c|}
\hline $1.52 \cdot 107$ & 1.06 \\
\hline $1.53 \cdot 107$ & 1.1 \\
\hline $1.54 \cdot 107$ & 1.19 \\
\hline $1.56 \cdot 107$ & 1.36 \\
\hline $1.6 \cdot 107$ & 1.67 \\
\hline $1.65 \cdot 107$ & 2.26 \\
\hline $1.75 \cdot 107$ & 3.69 \\
\hline $2.05 \cdot 107$ & 10.12 \\
\hline 0 & 0 \\
\hline
\end{tabular}

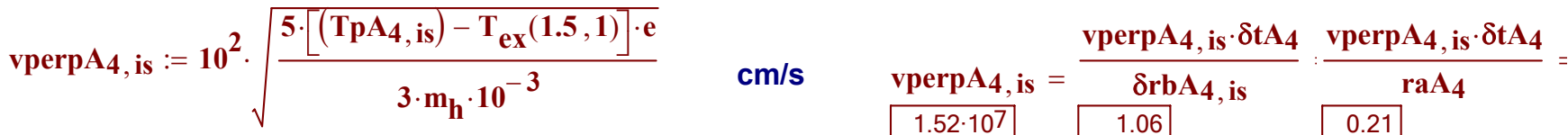

$$
\begin{aligned}
& \rho_{\mathbf{p A}_{4, \text { is }}}:=\rho \mathbf{p} \mathbf{A}_{4} \cdot\left(\frac{\operatorname{TpA_{4},1}}{\operatorname{Tp} \mathbf{A}_{4, \text { is }}}\right) \cdot\left(1+\frac{\operatorname{vperpA_{4},\text {is}} \cdot \delta \mathbf{t A}_{4}}{\operatorname{raA}_{4}}\right)^{-2} \\
& \text { and } \quad \rho_{\mathbf{p A}_{4,9}}:=\rho \mathbf{p A _ { 4 }} \\
& \operatorname{\rho r}_{\mathbf{p A}_{4, \text { is }}}:=\operatorname{\rho rpA_{4},\text {is}} \cdot\left(\frac{\operatorname{TpA} 4,1}{\operatorname{TpA} 4, \text { is }}\right) \cdot\left(1+\frac{\operatorname{vperpA_{4},\text {is}} \cdot \delta \mathbf{t A}_{4}}{\operatorname{raA}_{4}}\right)^{-2} \\
& \rho \mathbf{p A}_{4,9}:=\rho \operatorname{prp} \mathbf{A}_{4,9}
\end{aligned}
$$

\begin{tabular}{|r|}
\hline 0.21 \\
\hline 0.21 \\
\hline 0.21 \\
\hline 0.21 \\
\hline 0.22 \\
\hline 0.23 \\
\hline 0.24 \\
\hline 0.28 \\
\hline 0 \\
\hline
\end{tabular}

The corrected incident beam energy requirement adjusted for heated ablation plasma loss is

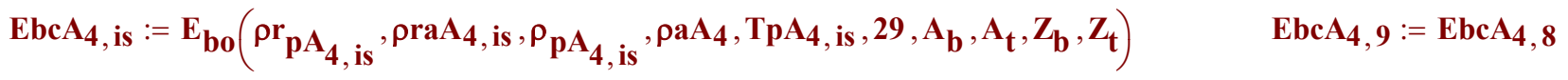




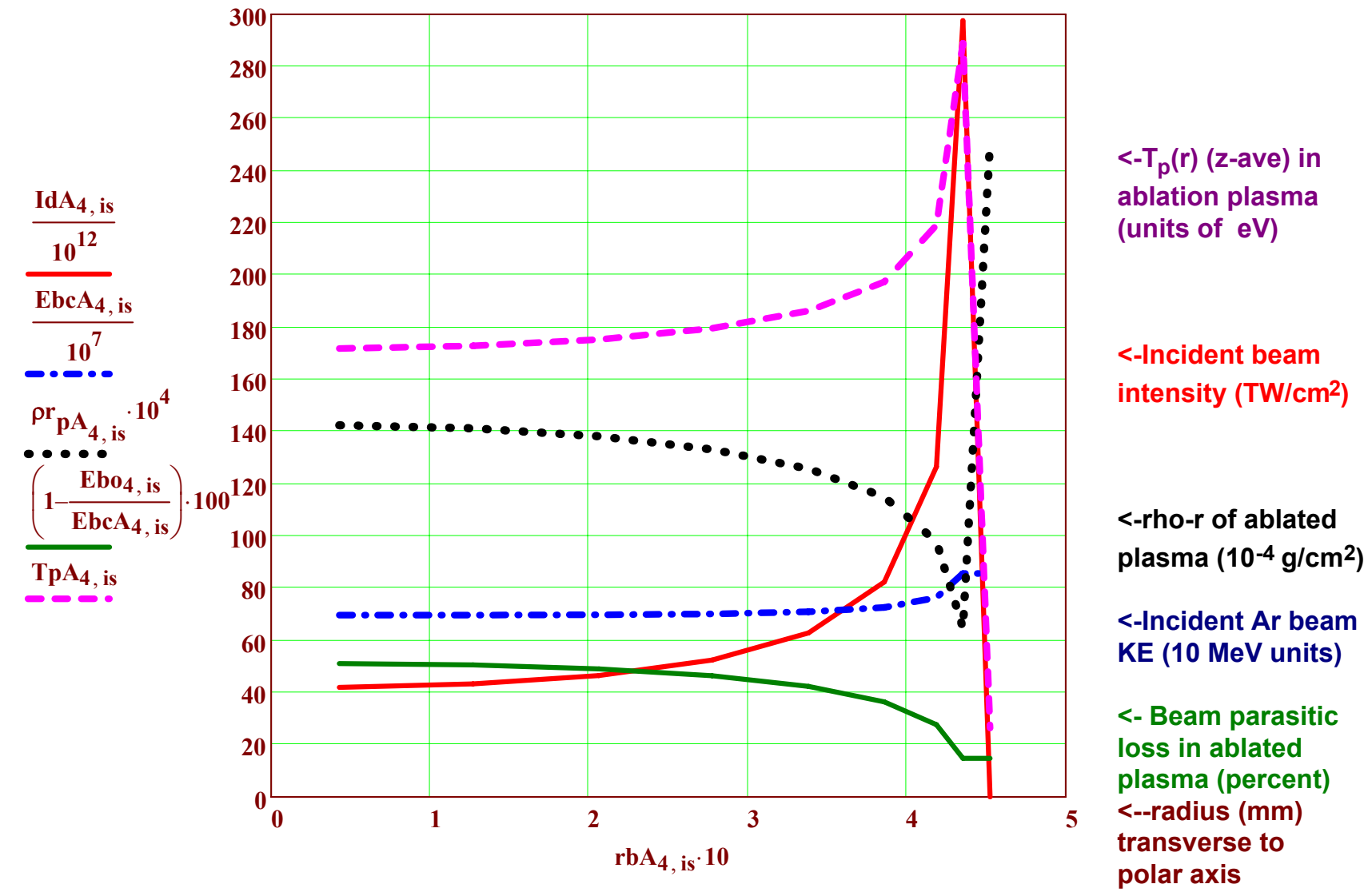

Figure 38: Plots of polar beam drive intensity (TW/ $\mathrm{cm}^{2}$, one of two sides), incident Ar beam energy (in $10 \mathrm{MeV}$ units), rho-r of ablated plasma column density $\left(10^{-4} \mathrm{~g} / \mathrm{cm}^{2}\right.$ units) (dotted black line), percent beam loss in ablated plasma, and the temperature $T_{p}$ of the ablation plasma (in eV units), as functions of radius in the beam channel, transverse to the polar axis near the target, during the fourth quarter of the ablation drive pulse for the large T-lean target case $A$.

$4^{\text {th }}$ qtr beam input inc loss on ablation plasma

$$
\text { EdcA }_{4}:=\sum_{\text {is }=1}^{8}\left[\frac{\text { EbcA }_{4}, \text { is }}{\text { Ebo }_{4}, \text { is }} \cdot 2 \cdot\left(\operatorname{WdA}_{4} \cdot \delta \mathrm{VaA}_{4} \text {, is } \cdot \operatorname{aA}_{4}\right)\right]
$$$$
\mathrm{EdA}_{4}=6.36 \times 10^{5}
$$$$
\text { -->fractional loss }
$$

Edrive $_{\text {tot }}=3.29 \times 10^{6}$

$$
\eta_{\text {dbAc }}:=\frac{\mathbf{E}_{\text {fuelA }}}{\text { Edrive }_{\text {tot }}}
$$

$$
\eta_{\text {dbAc }}=0.304
$$

$\operatorname{EdcA}_{4}=9.68 \times 10^{5} \quad J$

$\left(\operatorname{EdcA}_{4}-\operatorname{EdA}_{4}\right) \cdot\left(\operatorname{EdcA}_{4}\right)^{-1}=0.34$

Compressed fuel energy

$$
\mathbf{E}_{\text {fuelA }}:=1 \cdot 10^{6}
$$

(a new record > three times laser direct drive (if it holds up)

This increased overall coupling efficiency of $30 \%$ for a complete modelling of 2-D beam interaction with 2-D ablation plasma expansion, compares to $19 \%$ estimated for spherically-symmetric beam losses on ablation plasma (page 27), or compares to $25 \%$ taking 2-D effects only in expansion of ablation plasma (page 30). The highest ablation plasma temperatures $(300 \mathrm{eV})$ late in the drive pulse for the large targets (Case A) (Fig 38 above) is not high enough to cause significant range increase in for the $700 \mathrm{MeV}$ Ar beams (in view of Fig. 33), so all of the improvements in coupling efficiency in 2-D is due to beam geometry and plasma expansion alone. 

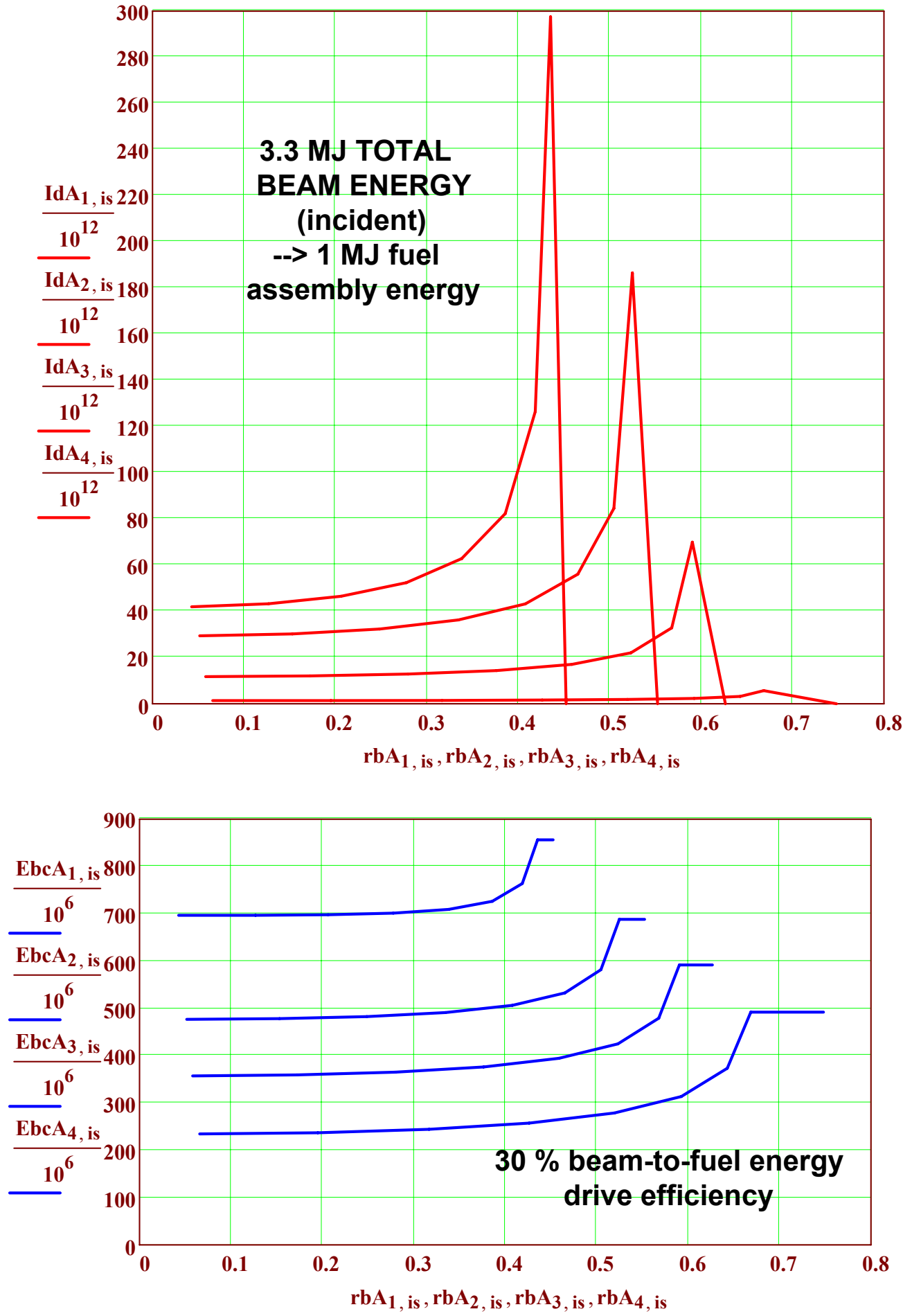

Beam intensity (one of two sides) (TW/ $\mathrm{cm}^{2}$ )

$<-@ \mathrm{t}=99$ ns

$<-@ t=93$ ns

$<-@ t=81$ ns

$<-@ \mathbf{t}=0$

<--beam radius at the target

Incident beam ion energy ( $\mathrm{MeV}$ ) $<-@ \mathrm{t}=99$ ns

$<-@ \mathrm{t}=93$ ns

$<-@ t=81$ ns

$<-@ \mathrm{t}=0$

<--beam radius at the target

Figure 39. Beam intensity profiles (TW/cm2) (one of two sides) (red curves-top) and beam ion energies for Argon (in MeV) (blue curves-bottom) vs radius for the large Case A reactor T-Lean DD target required for symmetric polar (two sided) drive with spherically symmetric ablators, at each time the $\mathrm{H} 2$ ablator loses $1 / 4$ of its initial mass. Beam losses in ablated plasma are accounted for in a 2-D model including heating Tp and "hole-boring" effects. Future work will seek use of P2 variations of ablator thickness $\Delta \operatorname{ra}(\theta)$ [shims] to enable time-dependent symmetry using beams with less-peaked rims. 
Beam requirements for DEMO Case B implosions with polar beam illumination

We now repeat the 2-D calculations that we did for Case $A$ for the small DEMO example (Case B).

Will more beam intensity at lower ion energies--> more range shortening (higher $T_{p}$, lower $\left.\rho_{p}\right)$ ?

$1^{\text {st }}$ quarter ablator mass $\quad \delta \mathrm{MaB}_{\mathrm{js}}:=(16.7-3.3) \cdot\left(4 \cdot 10^{3}\right)^{-1} \quad \delta \mathrm{MaB}_{1}=0.003 \quad$ g, @ density $\quad \rho \mathrm{B}_{1}:=\rho_{\mathrm{Ho}}$

1st qtr outer radius $\quad \operatorname{raB} 0:=0.371 \quad \mathrm{~cm}$, ablated over time interval $\quad \mathrm{tB}_{1}:=0 \quad \delta \mathrm{tB}_{1}:=32 \cdot 10^{-9} \quad \mathrm{~s}$

Ist qtr inner radius $\quad \mathrm{raB}_{1}:=\left[\mathrm{raB}^{3}-\left(\frac{3 \cdot \delta \mathrm{MaB}_{1}}{4 \cdot \pi \cdot \mathrm{\rho aB}_{1}}\right)\right]^{0.333} \quad \mathrm{~cm} \quad \mathrm{raB}_{1}=0.35 \quad \mathrm{~cm} \quad \delta \mathrm{raB}_{1}:=\mathrm{raB}-\mathrm{raB}_{1}$

1st qtr layer thickness $\delta \mathrm{raA}_{1}=\mathbf{0 . 0 3 8} \mathrm{cm}$. Radial KE/exhaust energy efficiency $\quad \eta_{\text {ex }}(1.5,0.2)=0.63$

1st qtr drive energy $\quad \mathrm{EdB}_{1}:=7.75 \cdot 10^{4} \cdot \eta_{\mathrm{ex}}(1.5,0.2)^{-1} \quad \mathrm{EdB}_{1}=1.2 \times 10^{5} \quad \mathrm{~J}$. Power: $\quad \operatorname{PdB}_{1}:=\mathrm{EdB}_{1} \cdot\left(\delta \mathrm{tB}_{1}\right)^{-1}$

$\mathrm{PdB}_{1} \cdot 10^{-12}=3.87 \quad$ TW. 1st qtr energy density $\quad \mathrm{WdB}_{1}:=\mathrm{EdB}_{1} \cdot\left(\delta \mathrm{MaB}_{1}\right)^{-1} \quad \mathrm{WdB}_{1}=3.7 \times 10^{7} \quad \mathrm{~J} / \mathrm{g}$

Ist quarter ablation front pressure $\quad \mathrm{WdB}_{1} \cdot \mathrm{\rho aB}_{1} \cdot 10^{6} \cdot 2^{-1} \cdot 10^{-11}=18.5 \quad \mathrm{MB} \quad \mathrm{WdB}_{1} \cdot \frac{\mathrm{m}_{\mathrm{h}}}{10 \cdot \mathrm{e}}=39 \quad \mathrm{eV}$

equiv.

1 st qtr shell volume $\quad \delta \mathrm{VaB}_{1}$, is $:=2 \cdot \pi \cdot\left(\operatorname{raB}_{1}+\frac{\delta \mathrm{raB}_{1}}{2}\right)^{2} \cdot \delta \mathrm{raB}_{1} \cdot \sin \left(\theta_{\text {is }}+\frac{\pi}{32}\right) \cdot \frac{\pi}{16} \quad \mathrm{~cm}^{3}$

2. $\sum_{\text {is }=1}^{8} \delta \mathrm{VaB}_{1}$, is $=0.033 \quad \mathrm{~cm}^{3} \quad \frac{4}{3} \cdot \pi \cdot\left[\left(\mathrm{raB}_{1}+\delta \mathrm{raB}_{1}\right)^{3}-\left(\mathrm{raB}_{1}\right)^{3}\right]=0.033 \quad$ Shell volume checks OK!

Beam convergence angle $\quad \theta b_{\text {is }}:=0.125 \cdot \sin \left(\theta_{\text {is }}\right) \quad$ Beam-pathlength-1st qtr $\quad \delta z^{2} B_{1}$, is $:=\frac{\delta \mathrm{raB}_{1}}{\cos \left(\theta_{\text {is }}-\theta b_{\text {is }}\right)}$

Beam range $\quad \rho \mathrm{raB}_{1}$, is $:=\rho \mathrm{aB}_{1} \cdot \delta \mathrm{zaB}_{1}$, is $\quad$ \& energy $\mathrm{EbB}_{1, \text { is }}:=\mathbf{E}_{\mathbf{b f}}\left(\rho \mathrm{B}_{1}, \rho \mathrm{BaB}_{1}\right.$, is $\left., 0.01,39, \mathbf{A}_{\mathbf{b}}, \mathbf{A}_{\mathbf{t}}, \mathbf{Z}_{\mathbf{b}}, \mathbf{Z}_{\mathbf{t}}\right)$

Incident beam radius vs polar angle $\theta \quad \mathrm{rbB}_{1}$, is $:=\operatorname{raB}_{1} \cdot \sin \left(\theta_{\text {is }}+\frac{\pi}{32}\right) \quad \quad \operatorname{rbB}_{1}, 9:=\operatorname{raB} 0+\delta z_{1} B_{1}, 9 \cdot \theta b 9$

Beam illumination width (cm) per $\theta$ increment $\quad \delta \mathrm{rbB}_{1}$, is $:=\left(\mathrm{raB}_{1}+\frac{\delta \mathrm{raB}_{1}}{2}\right) \cdot \sin \left(\theta_{\text {is }}+\frac{\pi}{16}\right)-\mathrm{raB}_{1} \cdot \sin \left(\theta_{\mathrm{is}}\right)$

Beam deposition intensity $\left(r_{b}\right) \quad \operatorname{IdB}_{1}$, is $:=\mathrm{WdB}_{1} \cdot \delta \mathrm{VaB}_{1}$, is $\cdot \operatorname{\rho aB}_{1} \cdot\left(\delta \mathrm{tB}_{1} \cdot 2 \cdot \pi \cdot \operatorname{rbB}_{1} \text {, is } \cdot \delta \mathrm{rbB}_{1} \text {, is }\right)^{-1} \quad \operatorname{IdB}_{1,9}:=0$

Table 19: 2-D polar drive requirements for DEMO Case $B, 1^{\text {st }}$ quarter ablation period. $\mathrm{EbB}_{1,9}:=\mathrm{EbB}_{1,8}$

\begin{tabular}{|c|c|c|c|c|c|}
\hline$\theta_{\text {is }}=$ & $\operatorname{rbB}_{1}$, is $=$ & $\delta \mathrm{zaB}_{1}$, is $=$ & $\operatorname{\rho raB}_{1, \text { is }}=$ & $\mathrm{IdB}_{1, \text { is }} \cdot 10^{-12}=$ & $\mathbf{E b B}_{\mathbf{1}}$, is $\cdot \mathbf{1 0}^{-\mathbf{6}}=$ \\
\hline 0 & 0.03 & 0.02 & 0.002 & 2.4 & 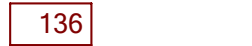 \\
\hline 0.2 & 0.1 & 0.02 & 0.002 & 2.4 & 137 \\
\hline 0.39 & 0.17 & 0.02 & 0.0021 & 2.6 & 142 \\
\hline 0.59 & 0.22 & 0.02 & 0.0023 & \begin{tabular}{|l|}
2.8 \\
\end{tabular} & 152 \\
\hline 0.79 & 0.27 & 0.03 & 0.0026 & 3.3 & 167 \\
\hline 0.98 & 0.31 & 0.03 & 0.0031 & 4.1 & 191 \\
\hline 1.18 & 0.34 & 0.04 & 0.0041 & 5.7 & 233 \\
\hline 1.37 & 0.35 & 0.06 & 0.0064 & \begin{tabular}{ll|}
10.1 \\
\end{tabular} & 317 \\
\hline 1.57 & 0.39 & 0.16 & 0.0161 & 0 & 317 \\
\hline $\begin{array}{l}\text { Polar } \\
\text { angle (rad) }\end{array}$ & $\begin{array}{l}\text { Beam } \\
\text { radius }(\mathrm{cm})\end{array}$ & $\begin{array}{l}\text { Shell } \\
z \text { depth }(\mathrm{cm})\end{array}$ & $\begin{array}{l}\text { Beam } \\
\text { range } \mathbf{g} / \mathrm{cm}^{2}\end{array}$ & $\begin{array}{l}\text { Beam } \\
\text { Intensity } \mathrm{TW} / \mathrm{cm}^{2}\end{array}$ & $\begin{array}{ll}\text { Argon Beam } \\
\text { Energy }(\mathrm{MeV})\end{array}$ \\
\hline
\end{tabular}

Page 62 
$\sum_{\text {is }=1}^{8} \delta \mathrm{rbB}_{1}$, is $=0.407 \quad \mathrm{~cm} \quad 2 \cdot \sum_{\text {is }=1}^{8}\left(\mathrm{WdB}_{1} \cdot \delta \mathrm{VaB}_{1}\right.$, is $\left.\cdot \rho \mathrm{aB} 1\right)=1.2 \times 10^{5} \quad \mathrm{~J}$-Energy Checks-OK!

$\sum_{\text {is }=1}^{8}\left[2 \cdot \pi \cdot\left(\mathrm{rbB}_{1}\right.\right.$, is $) \cdot \delta \mathrm{rbB}_{1}$, is $]=0.48 \quad \mathrm{~cm}^{2} \quad \pi \cdot \mathrm{raB0}^{2}=0.43 \quad \mathrm{~cm}^{2}$. OK as converging beam sees $>2 \pi$

Now lets correct for ablation plasma in this ablation period of $\frac{\delta \mathrm{tB}_{1}}{-9}=32$

The first quarter ablated plasma mass expands in this time interval to a radius $10^{-9}$ uimpB $1:=2.6 \cdot 10^{6} \mathrm{~cm} / \mathrm{s}$

average expansion velocity $\quad \mathrm{rpB}_{1}:=\mathrm{raB} 0+\left(\mathrm{u}_{\mathrm{ex}}(1.5,0.2)-\mathrm{uimpB} 1\right) \cdot \delta \mathrm{tB}_{1} \quad \mathrm{~cm} \quad \mathrm{rpB} 1=0.98 \quad \mathrm{~cm}$ and to a mass density $\quad \rho p B_{1}:=\delta \mathrm{MaB}_{1} \cdot\left[\frac{4}{3} \cdot \pi \cdot\left[\left(\mathrm{rpB}_{1}\right)^{3}-\mathrm{raB}^{3}\right]\right]^{-1} \quad \rho B_{1}=9.1 \times 10^{-4} \mathrm{~g} / \mathrm{cm}^{-4}$ and to a rho-r $\quad \operatorname{prpB}_{1}:=\rho \mathrm{pB}_{1} \cdot\left(\operatorname{rpB}_{1}-\mathrm{raB} 0\right) \quad \rho r p B_{1}=5.51 \times 10^{-4} \quad \mathrm{~g} / \mathrm{cm}^{2}, \sim 8 \%$ of the ave first quarter beam range.

Lets make a first order correction to the exhaust plasma temperature, assuming half of the incremental beam energy deposited into the ablation plasma mass within the beam channel

$$
\Delta \mathbf{E b p B}_{1, \text { is }}:=\left(\mathbf{E}_{\mathbf{b o}}\left(\rho \operatorname{prpB}_{1}, \rho \mathrm{aB}_{1} \text {, is }, \rho \mathrm{B}_{1}, \rho \mathrm{BB}_{1}, 150,39, \mathbf{A}_{\mathbf{b}}, \mathbf{A}_{\mathbf{t}}, \mathbf{Z}_{\mathbf{b}}, \mathbf{Z}_{\mathbf{t}}\right)-\mathbf{E b B}_{1}, \text { is }\right) \cdot\left(\mathbf{E b B}_{1}, \text { is }\right)^{-1}
$$

goes into increased hydro motion (both radial and transverse), the other half into incremental thermal energy $3 \Delta T_{p}$, and for the moment lets assume the ablated plasma density does not change; then we have

$$
\begin{aligned}
\operatorname{TpB}_{1, \text { is }}:= & \frac{\operatorname{IdB}_{1, \text { is }} \cdot \delta \mathrm{tB}_{1} \cdot \Delta \mathrm{EbpB}_{1}, \text { is }}{\rho \operatorname{\rho pB} B_{1}} \cdot \frac{\mathrm{m}_{\mathbf{h}}}{6 \cdot \mathrm{e}} \ldots \\
& +\mathbf{T}_{\mathbf{e x}}(1.5,0.2)
\end{aligned}
$$

Table 20: Effects of beam heating of ablation plasma as a funtion of polar angle $\theta$ in the beam channel

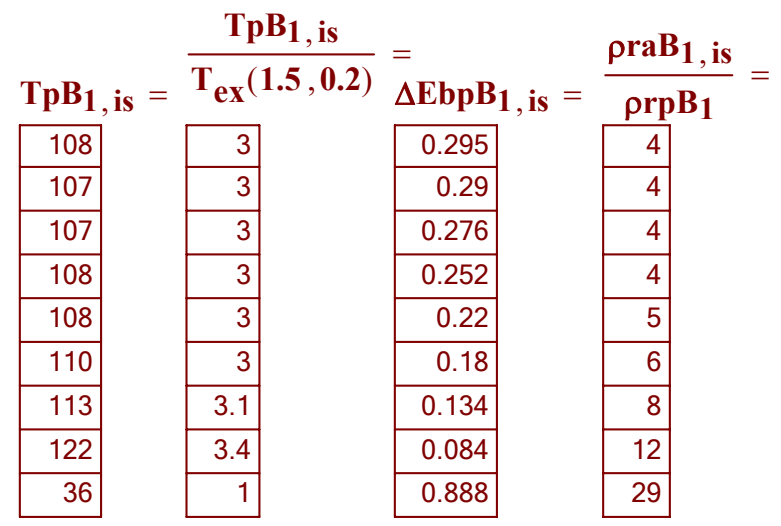

One can see in this table that the ablation plasma is substantially heated (Te increases several-fold) during the 1 st qtr of the pulse. 
The next question to ask is, does the heating (pressurization) cause significant enhanced expansion in the shorter transverse to the beam channel (polar axis) direction? One estimate is the fractional radial expansion of ablated plasma possible in the 1st qtr time of $\delta \mathrm{tB}$, following eq77:

$\operatorname{vperp}_{1} 1$, is $:=10^{2} \cdot \sqrt{\frac{5 \cdot\left[\left(\mathrm{TpB}_{1}, \text { is }\right)-\mathrm{T}_{\mathrm{ex}}(1.5,0.2)\right] \cdot \mathrm{e}}{3 \cdot \mathrm{m}_{\mathrm{h}} \cdot 10^{-3} \mathrm{~cm} / \mathrm{s}}}$

Table 21: Displacement of ablated plasma by beam heatin!

Table 15 showing vperp* $\delta t / \delta$ rb $\gg>1$ means that beam heating of ablation plasma will reach pressure equilibrium locally within the beam channel very quickly, and vperp* $\delta$ t/ $r a \sim 1$ means significant expansion transverse to the polar axis over the whole channel, justifying allocating half the incremental beam energy input going into hydro motion. The first effect of local pressure balance means the local

$\operatorname{vperp}_{1} \mathrm{~B}_{1}$ is $=$

\begin{tabular}{|r|}
\hline $1.07 \cdot 107$ \\
\hline $1.07 \cdot 107$ \\
\hline $1.07 \cdot 107$ \\
\hline $1.07 \cdot 107$ \\
\hline $1.07 \cdot 107$ \\
\hline $1.08 \cdot 107$ \\
\hline $1.11 \cdot 107$ \\
\hline $1.17 \cdot 107$ \\
\hline 0 \\
\hline
\end{tabular}

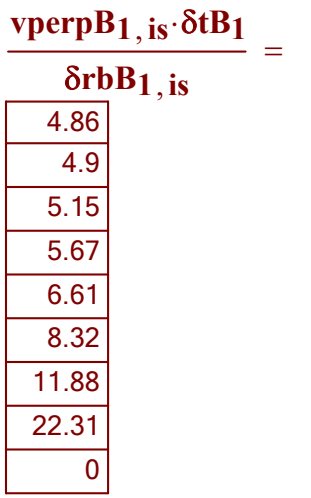

$\frac{\text { vperp } \mathbf{B}_{1}, \text { is } \cdot \delta \mathbf{t B}_{\mathbf{1}}}{\mathbf{r a B 0}}=$
\begin{tabular}{|r|}
\hline 0.92 \\
\hline 0.92 \\
\hline 0.92 \\
\hline 0.92 \\
\hline 0.93 \\
\hline 0.94 \\
\hline 0.96 \\
\hline 1.01 \\
\hline 0 \\
\hline
\end{tabular}

ablation plasma density will be depressed inversely with the local increase in beam temperature. The second effect will reduce the overall pressure withing the beam channel by roughly a factor of $(1+v p e r p * \delta t / r a)^{\wedge}-1$ (The net $T_{p}$ will stay roughly the same as beam energy $d E / d x$ transfer as "per electron"remains rougly the same. Using "pressure balance" and channel expansion factors, the corrected ablation plasma densities and associated rho-r's are reduced by beam heating are estimated by

$$
\begin{aligned}
& \rho_{\mathrm{pB}_{1, \text { is }}}:=\rho \mathrm{pB}_{1} \cdot\left(\frac{\mathrm{TpB}_{1,1}}{\mathrm{TpB}_{1, \text { is }}}\right) \cdot\left(1+\frac{\mathrm{vperpB}_{1}, \text { is } \cdot \delta \mathrm{tB}_{1}}{\mathrm{raB} 0}\right)^{-2} \quad \rho_{\mathrm{pB}_{1,9}}:=\rho \mathrm{pB}_{1} \\
& \text { and } \quad \rho \mathrm{pB}_{1, \text { is }}:=\operatorname{\rho rpB} B_{1} \cdot\left(\frac{\mathrm{TpB}_{1,1}}{\mathrm{TpB}_{1}, \text { is }}\right) \cdot\left(1+\frac{\mathrm{vperpB}_{1, \text { is }} \cdot \delta \mathrm{tB}_{1}}{\operatorname{raB} 0}\right)^{-2} \quad \rho \mathrm{p}_{\mathrm{pB}_{1,9}}:=\rho \operatorname{rpB}_{1}
\end{aligned}
$$

We can now plot all these adjusted beam requirements versus beam radius in the next Figure 35 . The corrected incident beam energy requirement adjusted for heated ablation plasma loss is

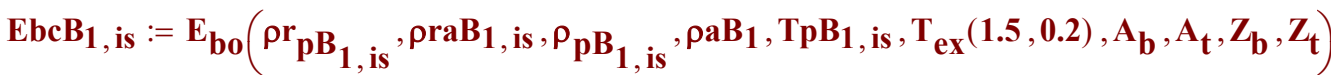

$$
\begin{aligned}
& \text { Page } 64 \\
& \operatorname{EbcB}_{1,9}:=\operatorname{EbcB}_{1,8}
\end{aligned}
$$




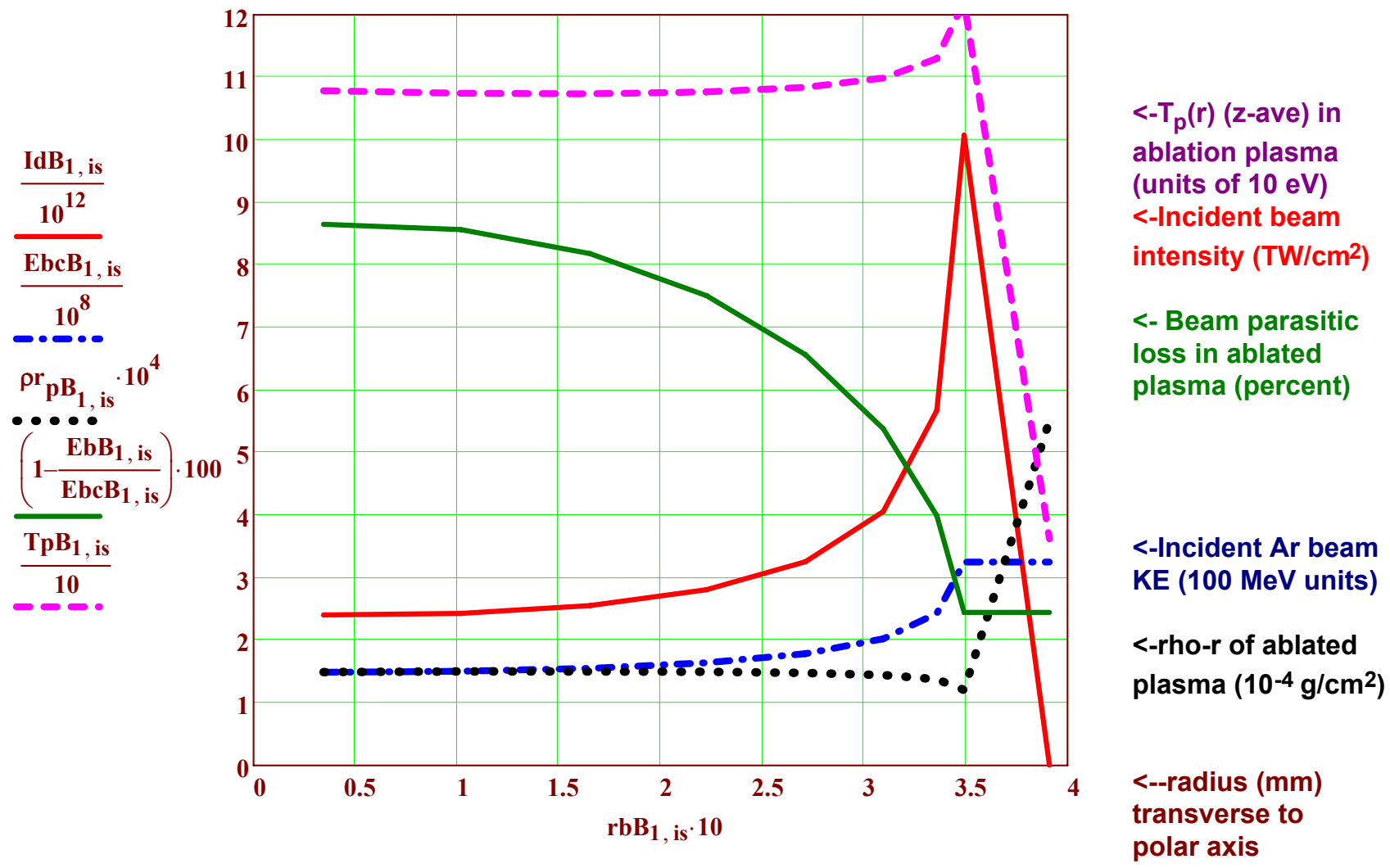

Figure 40: Plots of polar beam drive intensity (TW/ $\mathrm{cm}^{2}$, one of two sides), incident Ar beam energy (in $100 \mathrm{MeV}$ units), rho-r of ablated plasma column density $\left(10^{-4} \mathrm{~g} / \mathrm{cm}^{2}\right.$ units) (dotted black line), percent beam loss in ablated plasma, and the temperature $T_{p}$ of the ablation plasma(units of $10 \mathrm{eV}$ ), as

functions of radius in the beam channel, transverse to the polar axis near the target, during the first quarter of the ablation drive pulse, (the foot part of the pulse) for the DEMO case B (see Figs 25 to 27 above for case $B$ details). Note required beam intensity is sharply higher (peak is $\sim 4 X$ intensity on axis) in the beam channel "rim", as expected due to the polar drive geometry shown in Fig. 32. Also, note the local ablation temperature $\left(T_{p}\left(r_{b}\right)\right.$ increases with the beam intensity, resulting in pressurization digging a "hole" in ablated plasma rho-r just in the annulus through which most beam energy is delivered, reducing parasitic beam loss. This beneficial effect will increase later in the drive.

$\begin{aligned} & \text { 1st qtr beam input inc } \\ & \text { loss on ablation plasma }\end{aligned} \quad \mathrm{EdcB}_{1}:=\sum_{\text {is }=1}^{8}\left[\frac{\mathrm{EbcB}_{1}, \text { is }}{\mathrm{EbB}_{1}, \text { is }} \cdot 2 \cdot\left(\mathrm{WdB}_{1} \cdot \delta \mathrm{VaB}_{1}\right.\right.$, is $\left.\left.\cdot \rho \mathrm{aB}_{1}\right)\right] \quad \quad \mathrm{EdcB}_{1}=1.29 \times 10^{5} \mathrm{~J}$ Neglecting beam ablation loss $\quad \mathrm{EdB}_{1}=1.24 \times 10^{5} \quad-\rightarrow$ fractional loss $\left(\mathrm{EdcB}_{1}-\mathrm{EdB}_{1}\right) \cdot\left(\mathrm{EdcB}_{1}\right)^{-1}=0.04$

Fig. 40 shows a key feature of polar drive geometry-the local peaking of beam intensity and locally higher beam ion energy in the "rim" of the beam channel driving the limb of the ablator shell. This ideal beam variation provides symmetric ablation drive for a spheical implosion, but may prove difficult to achieve in practice, and so further work will explore ways to relax the locally-sharp, beam intensity "rim":

(a) Most important, add appropriate $\Delta \mathrm{ra}(\theta)$ capsule ablator shimming to accept a more uniform beam profile;

(b) Allow 20\% low mode-P2 asymmetries at large rho-r fuel (Steve Slutz at San Ramon IFE meeting);

(c) Possibly in conjuction with (a), overdrive the foot intensity at the rim for early 5 to $20 \%$ P2 prolate asymmetry with beam spill beyond the limb (small drive energy penalty, and then under-drive the rim later; (d) If ignition still fails in a too-asymmetric implosion, then add a powerful late shock. 
Polar drive parameters for Case $B$, 2nd quarter of ablation drive

$2^{\text {nd }}$ quarter ablator mass $\quad \delta \mathrm{MaB}_{2}=0.003 \quad \mathrm{tB}_{2}:=\delta \mathrm{tB}_{1} \quad \mathrm{tB}_{2}=3.2 \times 10^{-8} \quad \mathrm{~g}$, @ density $\quad \rho_{\mathrm{aB}}:=3 \cdot \rho_{\mathrm{Ho}}$ $2^{\text {nd }}$ qtr outer radius $\quad \mathrm{raB} 1:=0.325 \quad \mathrm{~cm}$, ablated over time interval $\delta \mathrm{tB}_{2}:=4.9 \cdot 10^{-9} \mathrm{~s}$

2nd qtr inner radius $\quad \mathrm{raB}_{2}:=\left[\mathrm{raB1}^{3}-\left(\frac{3 \cdot \delta \mathrm{MaB}_{2}}{4 \cdot \pi \cdot \rho \mathrm{B}_{2}}\right)\right]^{0.333} \quad \mathrm{raB}_{2}=0.317 \quad \delta \mathrm{raB}_{2}:=\mathrm{raB} 1-\mathrm{raB}_{2}$

$2^{\text {nd }}$ qtr layer thickness $\delta \mathrm{raB}_{2}=0.008 \quad \mathrm{~cm}$. Radial KE/exhaust energy efficiency $\eta_{\mathrm{ex}}(1.5,0.2)=0.63$

$2^{\text {nd }}$ qtr drive energy $\quad \mathrm{EdB}_{2}:=7.75 \cdot 10^{4} \cdot \eta_{\mathrm{ex}}(1.5,0.2)^{-1} \quad \mathrm{EdB}_{2}=1.24 \times 10^{5} \quad$ J. Power: $\quad \operatorname{PdB}_{2}:=\mathrm{EdB}_{2} \cdot\left(\delta \mathrm{tB}_{2}\right)^{-1}$ $\mathrm{PdB}_{2} \cdot 10^{-12}=25 \quad$ TW. 2nd qtr energy density $\quad \mathrm{WdB}_{2}:=\mathrm{EdB}_{2} \cdot\left(\delta \mathrm{MaB}_{2}\right)^{-1} \quad \mathrm{WdB}_{2}=3.69 \times 10^{7} \quad \mathrm{~J} / \mathrm{g}$ $2^{\text {nd }}$ quarter ablation front pressure $\quad \mathrm{WdB}_{2} \cdot \rho \mathrm{aB}_{2} \cdot 10^{6} \cdot 2^{-1} \cdot 10^{-11}=55.4 \quad \mathrm{MB} \quad \mathrm{WdB}_{2} \cdot \frac{\mathrm{m}_{\mathrm{h}}}{10 \cdot \mathrm{e}}=39 \quad \mathrm{eV}$ $2^{\text {nd }}$ qtr shell volume $\quad \delta \mathrm{VaB}_{2}$, is $:=2 \cdot \pi \cdot\left(\mathrm{raB}_{2}+\frac{\delta \mathrm{raB}_{2}}{2}\right)^{2} \cdot \delta \mathrm{raB}_{2} \cdot \sin \left(\theta_{\mathrm{is}}+\frac{\pi}{32}\right) \cdot \frac{\pi}{16} \quad \mathrm{~cm}^{3}$ 2. $\sum_{\text {is }=1}^{8} \delta \mathrm{VaB}_{2}$, is $=0.011 \quad \mathrm{~cm}^{3} \quad \frac{4}{3} \cdot \pi \cdot\left[\left(\mathrm{raB}_{2}+\delta \mathrm{raB}_{2}\right)^{3}-\left(\mathrm{raB}_{2}\right)^{3}\right]=0.011 \quad$ Shell volume checks OK! Beam convergence angle $\quad \theta b_{\text {is }}:=0.125 \cdot \sin \left(\theta_{\text {is }}\right) \quad$ Beam-pathlength-2nd qtr $^{\text {is }=1} \quad \delta z^{2} B_{2}$, is $:=\frac{\delta \operatorname{raB}_{2}}{\cos \left(\theta_{\text {is }}-\theta b_{\text {is }}\right)}$ Beam range $\quad \rho r B_{2}$, is $:=\rho a_{2} \cdot \delta z_{2} B_{2}$, is $\quad$ \& energy $\quad \mathbf{E b B}_{2}$, is $:=\mathbf{E}_{\mathbf{b f}}\left(\rho \mathbf{a B}_{2}, \rho r a B_{2}\right.$, is $\left., 0.01,39, \mathbf{A}_{\mathbf{b}}, \mathbf{A}_{\mathbf{t}}, \mathbf{Z}_{\mathbf{b}}, \mathbf{Z}_{\mathbf{t}}\right)$ Incident beam radius vs polar angle $\theta \quad \mathrm{rbB}_{2}$, is $:=\mathrm{raB}_{2} \cdot \sin \left(\theta_{\mathrm{is}}+\frac{\pi}{32}\right) \quad \quad \mathrm{rbB}_{2}, 9:=\operatorname{raB} 1+\delta \mathrm{zaB}_{2}, 9 \cdot \theta \mathrm{bg}_{9}$ Beam illumination width (cm) per $\theta$ increment $\quad \delta \mathrm{rbB}_{2}$, is $:=\left(\mathrm{raB}_{2}+\frac{\delta \mathrm{raB}_{2}}{2}\right) \cdot \sin \left(\theta_{\text {is }}+\frac{\pi}{16}\right)-\mathrm{raB}_{2} \cdot \sin \left(\theta_{\mathrm{is}}\right)$ Beam deposition intensity $\left(r_{b}\right) \quad I d B_{2}$, is := $\mathrm{WdB}_{2} \cdot \delta \mathrm{VaB}_{2}$, is $\cdot \rho \mathrm{aB}_{2} \cdot\left(\delta \mathrm{tB}_{2} \cdot 2 \cdot \pi \cdot \mathrm{rbB}_{2} \text {, is } \delta \mathrm{rbB}_{2} \text {, is }\right)^{-1} \quad \mathrm{IdB}_{2,9}:=0$ $\mathrm{EbB}_{2}, 9:=\mathrm{EbB}_{2}, 8$

Table 22: 2-D polar drive requirements for Case B, $2^{\text {nd }}$ quarter ablation period.

\begin{tabular}{|c|c|c|c|c|c|}
\hline$\theta_{\text {is }}=$ & $\operatorname{rbB}_{2}$, is $=$ & $\delta \mathrm{zaB}_{2}$, is $=$ & $\operatorname{praB}_{2}$, is $=$ & $\mathrm{IdB}_{2}$, is $\cdot 10^{-12}=$ & $\mathrm{EbB}_{2}$, is $\cdot 10^{-6}=$ \\
\hline 0 & 0.03 & 0.008 & 0.0025 & 19 & 147 \\
\hline 0.2 & 0.09 & 0.008 & 0.0025 & 20 & 148 \\
\hline 0.39 & 0.15 & 0.009 & 0.0026 & 21 & 154 \\
\hline 0.59 & 0.2 & 0.01 & 0.0029 & 23 & 164 \\
\hline 0.79 & 0.24 & 0.011 & 0.0032 & 28 & 180 \\
\hline 0.98 & 0.28 & 0.013 & 0.0039 & 36 & 207 \\
\hline \begin{tabular}{ll|}
1.18 \\
\end{tabular} & 0.3 & 0.017 & 0.0051 & 54 & 252 \\
\hline 1.37 & 0.32 & 0.026 & 0.0079 & 117 & 343 \\
\hline 1.57 & 0.33 & 0.066 & 0.0199 & 0 & 343 \\
\hline $\begin{array}{l}\text { Polar } \\
\text { angle (rad) }\end{array}$ & $\begin{array}{l}\text { Beam } \\
\text { radius }(\mathrm{cm})\end{array}$ & $\begin{array}{l}\text { Shell } \\
z \text { depth }(\mathrm{cm})\end{array}$ & $\begin{array}{l}\text { Beam } \\
\text { range } \mathrm{g} / \mathrm{cm}^{2}\end{array}$ & $\begin{array}{l}\text { Beam } \\
\text { Intensity } \mathrm{TW} / \mathrm{cm}^{2}\end{array}$ & $\begin{array}{l}\text { Argon Beam Energy } \\
\text { (MeV) (No parasitic } \\
\text { ablation plasma loss) }\end{array}$ \\
\hline
\end{tabular}


Now lets correct for ablation plasma in this ablation period of The second quarter ablated plasma mass expands in this time interval to a radius $\mathrm{u}_{\text {aveB2 } 2}:=0.8 \cdot 10^{7} \quad \mathrm{rpB}_{2}:=\operatorname{raB} 1+\left(\mathrm{u}_{\mathrm{ex}}(1.5,0.2)-\mathrm{u}_{\mathrm{aveB} 2}\right) \cdot \delta \mathrm{tB}_{2} \quad \mathrm{rpB}_{2}=0.4 \quad \mathrm{~cm}$ $\frac{\delta \mathrm{tB}_{2}}{=4.9}$ ns and to a mass density $\quad \rho p_{2}:=\delta \mathrm{MaB}_{2} \cdot\left[\frac{4}{3} \cdot \pi \cdot\left[\left(\mathrm{rpB}_{2}\right)^{3}-\mathrm{raB}^{3}\right]\right]^{-1} \quad \rho \mathrm{pB}_{2}=0.031 \quad \mathrm{~g} / \mathrm{cm} 3$ and to a rho-r $\quad \rho r p B_{2}$, is $:=\rho p B_{2} \cdot\left(r p B_{2}-r a B 1\right)+\rho r_{p B}, \quad \rho r p A_{2}, 8=0.0041$ (Note we add ablation rho-r from the first qtr!) $\mathrm{g} / \mathrm{cm}^{2}$, now $\sim 80 \%$ of the ave $2^{\text {nd }}$ qtr ablator range.

Lets make a first order correction to the exhaust plasma temperature, assuming input goes both to hydro

$$
\Delta \mathbf{E b p B}_{2}, \text { is }:=\left(\mathbf{E}_{\mathbf{b o}}\left(\rho r p B_{2}, \text { is }, \rho r a B_{2}, \text { is }, \rho p B_{2}, \rho B_{2}, 190,39, \mathbf{A}_{\mathbf{b}}, \mathbf{A}_{\mathbf{t}}, \mathbf{Z}_{\mathbf{b}}, \mathbf{Z}_{\mathbf{t}}\right)-\mathbf{E b B}_{2}, \text { is }\right) \cdot\left(\mathbf{E b B}_{2}, \text { is }\right)^{-1}
$$

and into incremental thermal energy $3 \Delta T_{p}$, and for the moment lets assume the ablated plasma density does not change; then we have, adding the last qtr beam input to heating on top:

$$
\begin{aligned}
& \mathrm{TpB}_{2, \text { is }}:=\frac{\mathrm{IdB}_{2}, \text { is } \cdot \delta \mathrm{tB}_{2} \cdot \Delta \mathrm{EbpB} \mathrm{B}_{2}, \text { is }}{\rho r p B_{2}, \text { is }} \cdot \frac{\mathrm{m}_{\mathbf{h}}}{6 \cdot \mathrm{e}} \ldots \\
& \mathrm{TpB}_{2, \text { is }}=\frac{\mathrm{TpB}_{2, \text { is }}}{\mathrm{T}_{\mathrm{ex}}(1.5,0.2)}= \\
& +\mathrm{TpB}_{1} \text {, is } \\
& T_{\text {ex }}(1.5,0.2)=36.18 \\
& \begin{array}{|r|}
\hline 163 \\
\hline 163 \\
\hline 164 \\
\hline 166 \\
\hline 169 \\
\hline 174 \\
\hline 185 \\
\hline 221 \\
\hline 36 \\
\hline
\end{array} \\
& \begin{array}{|r|}
\hline 4.5 \\
\hline 4.5 \\
\hline 4.5 \\
\hline 4.6 \\
\hline 4.7 \\
\hline 4.8 \\
\hline 5.1 \\
\hline 6.1 \\
\hline 1 \\
\hline
\end{array}
\end{aligned}
$$

\begin{tabular}{|l|}
\hline 0.751 \\
\hline 0.739 \\
\hline 0.704 \\
\hline 0.645 \\
\hline 0.565 \\
\hline 0.465 \\
\hline 0.347 \\
\hline 0.217 \\
\hline 1.006 \\
\hline
\end{tabular}

\begin{tabular}{|c|c|c|}
\hline $1.42 \cdot 107$ & 1.11 & 0.22 \\
\hline $1.42 \cdot 107$ & 1.14 & 0.22 \\
\hline $1.43 \cdot 107$ & 1.23 & 0.22 \\
\hline $1.44 \cdot 107$ & 1.38 & 0.22 \\
\hline $1.46 \cdot 107$ & 1.67 & 0.23 \\
\hline $1.49 \cdot 107$ & 2.2 & 0.23 \\
\hline $1.54 \cdot 107$ & 3.43 & 0.24 \\
\hline $1.72 \cdot 107$ & 8.23 & 0.27 \\
\hline 0 & 0 & 0 \\
\hline
\end{tabular}

\begin{tabular}{|r|}
\hline $2.47 \cdot 10^{8}$ \\
\hline $2.5 \cdot 108$ \\
\hline $2.58 \cdot 108$ \\
\hline $2.73 \cdot 108$ \\
\hline $2.98 \cdot 108$ \\
\hline $3.38 \cdot 108$ \\
\hline $4.06 \cdot 108$ \\
\hline $5.41 \cdot 108$ \\
\hline $5.41 \cdot 108$ \\
\hline
\end{tabular}

One can see in this table that the ablation plasma is substantially heated (Te increases $6 \mathrm{X}$ ) (more in the 2 nd qtr of the pulse). Check if heating (pressurization) can still cause significant enhanced expansion in the shorter transverse to the beam channel (polar axis) direction in the shorter time $\delta$ tB $_{2}$ :

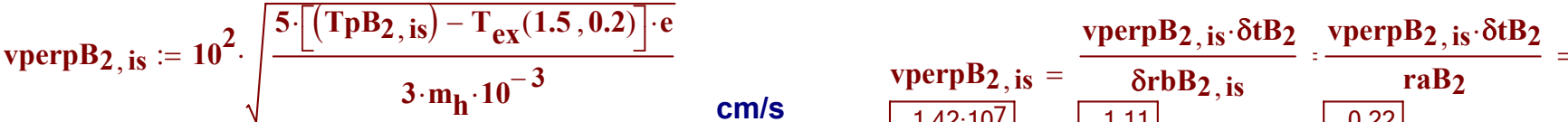

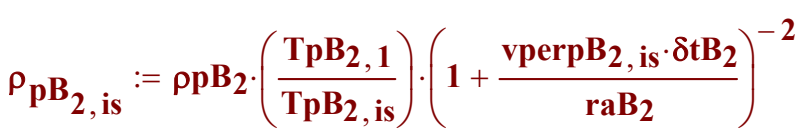

$$
\begin{aligned}
& \text { and } \quad \rho_{\mathrm{pB}_{2,9}}:=\rho \mathrm{pB}_{2} \\
& \operatorname{\rho r}_{\mathrm{pB}_{2, \text { is }}}:=\rho \operatorname{\rho rpB_{2},\text {is}} \cdot\left(\frac{\mathrm{TpB}_{2,1}}{\mathrm{TpB}_{2, \text { is }}}\right) \cdot\left(1+\frac{\operatorname{vperp}_{2}, \text { is } \cdot \delta \mathrm{tB}_{2}}{\mathrm{raB}_{2}}\right)^{-2} \\
& \rho \mathrm{pB}_{2,9}:=\rho \operatorname{prpB}_{2,9}
\end{aligned}
$$

The corrected incident beam energy requirement adjusted for heated ablation plasma loss is

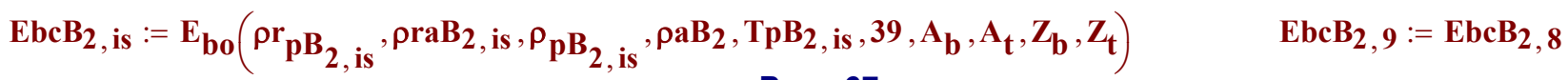




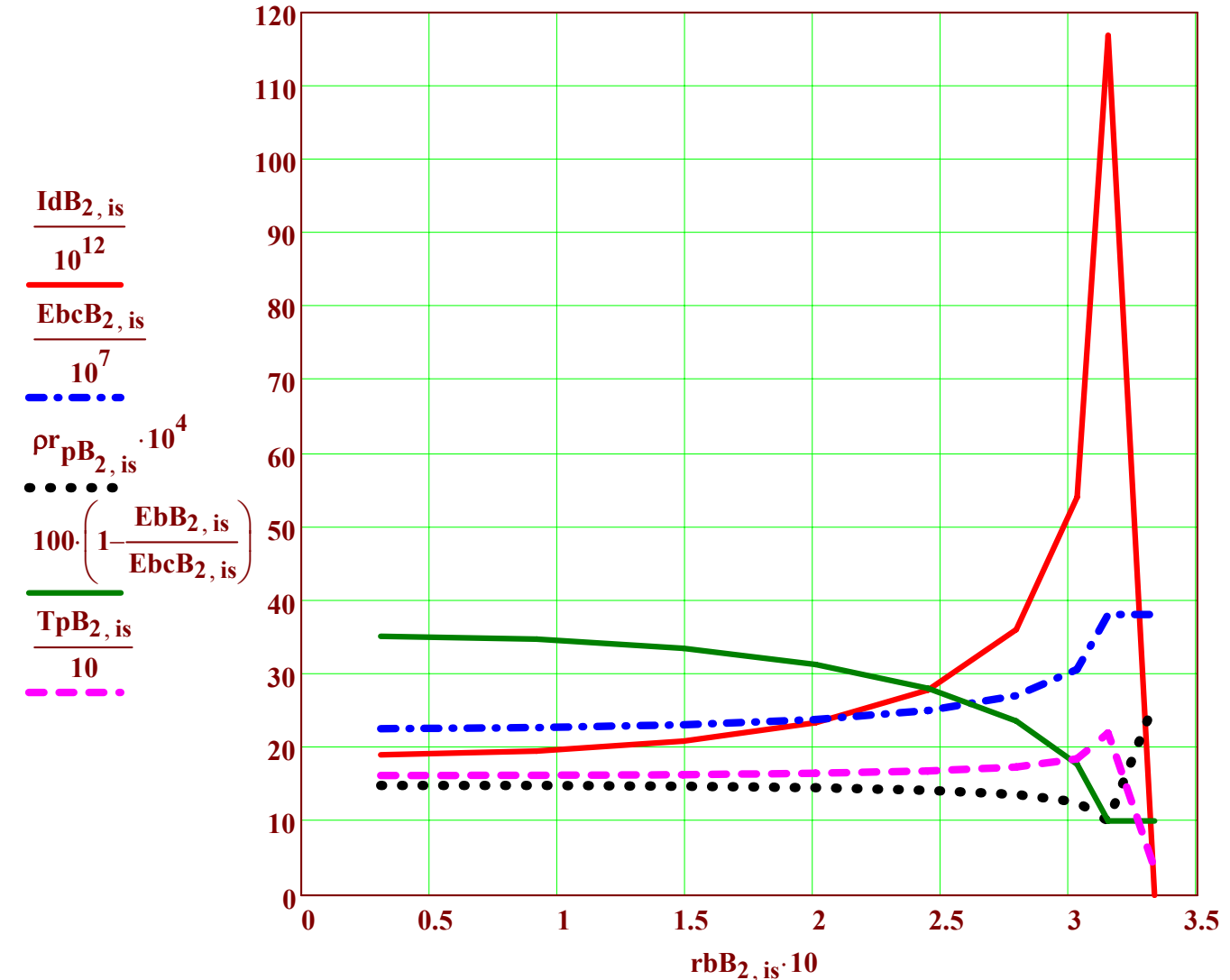

$<-I n c i d e n t$ beam intensity $\left(\mathrm{TW} / \mathrm{cm}^{2}\right.$ )

<- Beam parasitic loss in ablated plasma (percent) $<-$-Incident Ar beam KE (10 MeV units) $<-T_{p}(r)$ (z-ave) in ablation plasma (units of $10 \mathrm{eV}$ ) <-rho-r of ablated plasma $\left(10^{-4} \mathrm{~g} / \mathrm{cm}^{2}\right)$ $<-$ radius $(\mathrm{mm})$ transverse to polar axis

Figure 41: Plots of polar beam drive intensity (TW/ $\mathrm{cm}^{2}$, one of two sides), incident Ar beam energy (in $10 \mathrm{MeV}$ units), rho-r of ablated plasma column density $\left(10^{-4} \mathrm{~g} / \mathrm{cm}^{2}\right.$ units) (dotted black line), percent beam loss in ablated plasma, and the temperature $T_{p}$ of the ablation plasma (in $10 \mathrm{eV}$ units), as functions of radius in the beam channel, transverse to the polar axis near the target, during the second quarter of the ablation drive pulse for the DEMO case $B$. Note beam intensity peak is now $\sim 6 X$ intensity on axis, and percent beam loss on ablated plasma is higher. Also, note the local ablation temperature $\left(T_{p}\left(r_{b}\right)\right.$ has increased with the beam intensity, and a greater beam heating effect digging a hole in the density at the beam rim position, compared to the 1 st quarter ablation period.

$$
\begin{aligned}
& \begin{array}{l}
\text { 2nd qtr beam input inc } \\
\text { loss on ablation plasma }
\end{array} \quad \mathrm{EdcB}_{2}:=\sum_{\text {is }=1}^{8}\left[\frac{\mathrm{EbcB}_{2} \text {, is }}{\mathrm{EbB}_{2}, \text { is }} \cdot 2 \cdot\left(\mathrm{WdB}_{2} \cdot \delta \mathrm{VaB}_{2}, \text { is } \cdot \rho \mathrm{BB}_{2}\right)\right] \quad \quad \mathrm{EdcB}_{2}=1.57 \times 10^{5} \quad \mathrm{~J} \\
& \text { Neglecting beam ablation loss } \quad \mathrm{EdB}_{2}=1.24 \times 10^{5} \quad-\rightarrow \text { fractional loss }\left(\mathrm{EdcB}_{2}-\mathrm{EdB}_{2}\right) \cdot\left(\mathrm{EdcB}_{2}\right)^{-1}=0.21
\end{aligned}
$$


Polar drive parameters for Case $B, 3$ rd quarter of ablation drive $\quad \mathrm{tB}_{3}:=\delta \mathrm{tB}_{1}+\delta \mathrm{tB}_{2}$

3rd quarter ablator mass $\delta \mathrm{MaB}_{3}=0.0034 \quad \mathrm{~g} \quad \mathrm{tB}_{3}=3.69 \times 10^{-8} \mathrm{~s} \quad @$ density $\quad \rho^{\mathrm{B}} \mathrm{B}_{3}:=5 \cdot \rho_{\mathrm{Ho}}$

$3^{\text {rd }}$ qtr outer radius $\quad \mathrm{raB2}:=0.296 \quad \mathrm{~cm}, \delta \mathrm{MaA}_{3}$ ablated over time interval $\quad \delta \mathrm{tB}_{3}:=\mathbf{2 . 4 \cdot 1 0 ^ { - 9 }} \mathrm{s}$

3rd qtr inner radius $\quad \mathrm{raB}_{3}:=\left[\mathrm{raB}^{3}-\left(\frac{3 \cdot \delta \mathrm{MaB}_{3}}{4 \cdot \pi \cdot \rho \mathrm{aB}_{3}}\right)\right]^{0.333} \quad \mathrm{raB}_{3}=0.29 \quad \delta \mathrm{raB}_{3}:=\mathrm{raB}_{2}-\mathrm{raB}_{3}$

$3^{\text {rd }}$ qtr layer thickness $\quad \delta \mathrm{raB}_{3}=0.006 \quad \mathrm{~cm}$. Radial KE/exhaust energy efficiency $\eta_{\text {ex }}(1.5,0.2)=0.63$

$3^{\text {rd }}$ qtr drive energy $\quad \mathrm{EdB}_{3}:=7.75 \cdot 10^{4} \cdot \eta_{\mathrm{ex}}(1.5,0.2)^{-1} \quad \mathrm{EdB}_{3}=1.24 \times 10^{5} \quad$ J. Power: $\quad \mathrm{PdB}_{3}:=\mathrm{EdB}_{3} \cdot\left(\delta \mathrm{tB}_{3}\right)^{-1}$

$\mathrm{PdB}_{3} \cdot 10^{-12}=52 \quad$ TW. $3^{\text {rd }}$ qtr energy density $\quad \mathrm{WdB}_{3}:=\mathrm{EdB}_{3} \cdot\left(\delta \mathrm{MaB}_{3}\right)^{-1} \quad \mathrm{WdB}_{3}=3.69 \times 10^{7} \quad \mathrm{~J} / \mathrm{g}$

3rd quarter ablation front pressure $\quad \mathrm{WdB}_{3} \cdot \rho \mathrm{aB}_{3} \cdot 10^{6} \cdot 2^{-1} \cdot 10^{-11}=92 \quad \mathrm{MB} \quad \mathrm{WdB}_{3} \cdot \frac{\mathrm{m}_{\mathrm{h}}}{10 \cdot \mathrm{e}}=39 \mathrm{eV}$

$3^{\text {rd }}$ qtr shell volume $\quad \delta \mathrm{VaB}_{3}$, is $:=2 \cdot \pi \cdot\left(\mathrm{raB}_{3}+\frac{\delta \mathrm{raB}_{3}}{2}\right)^{2} \cdot \delta \mathrm{raB}_{3} \cdot \sin \left(\theta_{\text {is }}+\frac{\pi}{32}\right) \cdot \frac{\pi}{16} \quad \mathrm{~cm}^{3}$

2. $\sum_{\mathrm{is}}^{8} \delta \mathrm{VaB}_{3}$, is $=0.006 \quad \mathrm{~cm}^{3} \quad \frac{4}{3} \cdot \pi \cdot\left[\left(\mathrm{raB}_{3}+\delta \mathrm{raB}_{3}\right)^{3}-\left(\mathrm{raB}_{3}\right)^{3}\right]=0.006 \quad$ Shell volume checks OK!

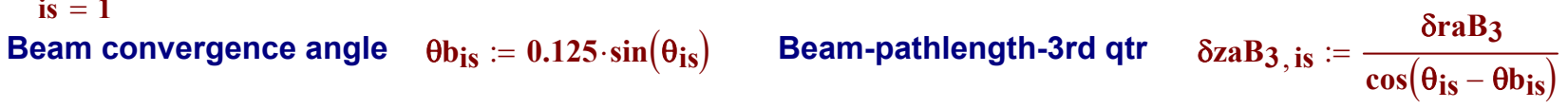

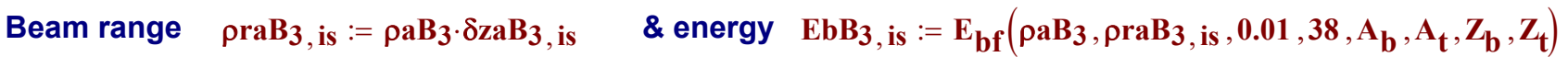

Incident beam radius vs polar angle $\theta \quad \mathrm{rbB}_{3}$, is $:=\mathrm{raB}_{3} \cdot \sin \left(\theta_{\mathrm{is}}+\frac{\pi}{32}\right) \quad \quad \mathrm{rbB}_{3}, 9:=\mathrm{raB} 2+\delta \mathrm{zaB}_{3}, 9 \cdot \theta \mathrm{b}_{9}$

Beam illumination width (cm) per $\theta$ increment $\quad \delta \mathrm{rbB}_{3}$, is $:=\left(\mathrm{raB}_{3}+\frac{\delta \mathrm{raB}_{3}}{2}\right) \cdot \sin \left(\theta_{\text {is }}+\frac{\pi}{16}\right)-\mathrm{raB}_{3} \cdot \sin \left(\theta_{\mathrm{is}}\right)$

Beam deposition intensity $\left(r_{b}\right) \quad I d B_{3}$, is $:=\mathrm{WdB}_{3} \cdot \delta \mathrm{VaB}_{3}$, is $\cdot \rho_{\mathrm{aB}} \cdot\left(\delta \mathrm{tB}_{3} \cdot 2 \cdot \pi \cdot \mathrm{rbB}_{3} \text {, is } \delta \mathrm{rbB}_{3} \text {, is }\right)^{-1} \quad \mathrm{IdB}_{3}, 9:=0$

$\mathrm{EbB}_{3}, \mathbf{9}:=\mathrm{EbB}_{3}, \mathbf{8}$

Table 23: 2-D polar drive requirements for Case B, $3^{\text {rd }}$ quarter ablation period.

\begin{tabular}{|c|c|c|c|c|c|}
\hline$\theta_{\text {is }}=$ & $\operatorname{rbB}_{3}$, is $=$ & $\delta \mathrm{zaB}_{3}$, is $=$ & $\operatorname{\rho raB}_{3}$, is $=$ & $\mathrm{IdB}_{3, \text { is }} \cdot 10^{-12}=$ & $\mathrm{EbB}_{3}$, is $\cdot 10^{-6}=$ \\
\hline 0 & 0.03 & 0.006 & 0.0029 & 46 & 160 \\
\hline 0.2 & 0.08 & 0.006 & 0.003 & 47 & 162 \\
\hline 0.39 & 0.14 & 0.006 & 0.0031 & 51 & 168 \\
\hline 0.59 & 0.18 & 0.007 & 0.0034 & 57 & 179 \\
\hline 0.79 & 0.22 & 0.008 & 0.0038 & 68 & 196 \\
\hline 0.98 & 0.26 & 0.009 & 0.0046 & 89 & 225 \\
\hline 1.18 & 0.28 & 0.012 & 0.006 & 135 & 274 \\
\hline 1.37 & 0.29 & 0.019 & 0.0093 & 308 & 372 \\
\hline 1.57 & 0.3 & 0.047 & 0.0235 & 0 & 372 \\
\hline $\begin{array}{l}\text { Polar } \\
\text { angle (rad) }\end{array}$ & $\begin{array}{l}\text { Beam } \\
\text { radius }(\mathrm{cm})\end{array}$ & $\begin{array}{l}\text { Shell } \\
z \text { depth }(\mathrm{cm})\end{array}$ & $\begin{array}{l}\text { Beam } \\
\text { range } \mathbf{g} / \mathrm{cm}^{2}\end{array}$ & $\begin{array}{l}\text { Beam } \\
\text { Intensity } \mathrm{TW} / \mathrm{cm}^{2}\end{array}$ & $\begin{array}{l}\text { Argon Beam Energy } \\
\text { (MeV) (Neglect parasitic } \\
\text { ablation plasma loss) }\end{array}$ \\
\hline
\end{tabular}

Page 69 
Now lets correct for ablation plasma in this ablation period of The third quarter ablated plasma mass expands in this time interval to a radius $\mathrm{u}_{\text {aveB3 } 3}:=1.55 \cdot 10^{7} \quad \operatorname{rpB}_{3}:=\operatorname{raB2}+\left(\mathrm{u}_{\mathrm{ex}}(1.5,0.2)-\mathrm{u}_{\mathrm{aveB} 3}\right) \cdot \delta \mathrm{tB}_{3} \quad \mathrm{rpB}_{3}=0.3 \quad \mathrm{~cm}$ and to a mass density $\rho \mathrm{pB}_{3}:=\delta \mathrm{MaB}_{3} \cdot\left[\frac{4}{3} \cdot \pi \cdot\left[\left(\mathrm{rpB}_{3}\right)^{3}-\mathrm{raB}^{3}\right]\right]^{-1}$

$$
\rho \mathrm{pB}_{3}=\mathbf{0 . 2 0 1} \mathrm{g} / \mathrm{cm} 3
$$

and to a rho-r $\quad \rho r p B_{3}$, is $:=\rho p B_{3} \cdot\left(\mathrm{rpB}_{3}-\mathrm{raB}_{2}\right)+\rho \mathrm{r}_{\mathrm{pB}_{2}, \text { is }} \quad \rho r p B_{3}, 8=0.0039$ (Note we add in ablation rho-r from the $2^{\text {nd }}$ qtr!) $\mathrm{g} / \mathrm{cm}^{2}$, now $\sim 95 \%$ of the ave $3^{\text {rd }}$ qtr ablator range.

Lets make a first order correction to the exhaust plasma temperature, assuming all beam energy deposited into the ablation plasma mass within the beam channel

$$
\Delta \mathbf{E b p B}_{3} \text {, is := }\left(\mathbf{E}_{\mathbf{b o}}\left(\rho r p B_{3} \text {, is }, \rho r B_{3}, \text { is }, \rho p B_{3}, \rho B_{3}, 200,36, A_{b}, A_{t}, Z_{b}, Z_{t}\right)-E_{b B}, \text { is }\right) \cdot\left(E_{b B}, \text { is }\right)^{-1}
$$

goes into incremental thermal energy $3 \Delta T_{p}$, and for the moment lets assume the ablated plasma density does not change; then we have, adding the last qtr beam input to heating on top :

$$
\begin{aligned}
& \mathrm{TpB}_{3}, \text { is }:=\frac{\mathrm{IdB}_{3}, \text { is } \cdot \delta \mathrm{tB}_{3} \cdot \Delta \mathrm{EbpB} \mathrm{B}_{3} \text { is }}{\rho \operatorname{rp} \mathrm{A}_{3}, \text { is }} \cdot \frac{\mathrm{m}_{\mathbf{h}}}{6 \cdot \mathrm{e}} \ldots \\
& +\mathrm{TpB}_{2} \text {, is } \\
& T_{\text {ex }}(1.5,0.2)=36.18
\end{aligned}
$$

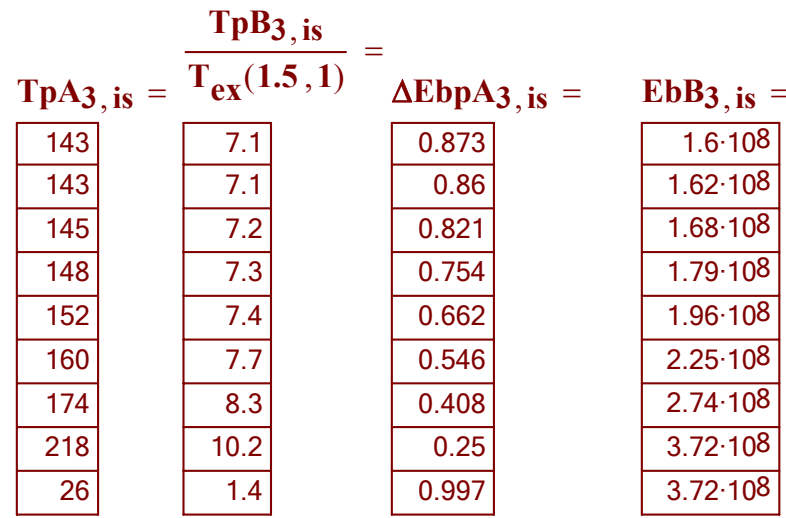

One can see in this table that the ablation plasma is substantially heated (Te increases 10X) (more in the 3rd qtr of the pulse). Check if heating (pressurization) can still cause significant enhanced expansion in the shorter transverse to the beam channel (polar axis) direction in the shorter time $\delta$ tB ${ }_{3}$ :

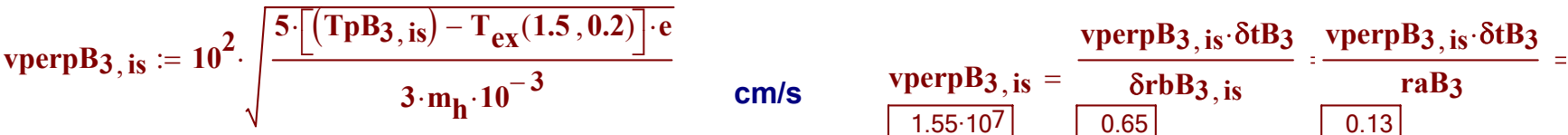

$$
\begin{aligned}
& \rho_{\mathbf{p B}_{3, \text { is }}}:=\rho p B_{3} \cdot\left(\frac{T B_{3}, 1}{T p B_{3}, \text { is }}\right) \cdot\left(1+\frac{\text { vperpB } B_{3, \text { is }} \cdot \delta B_{3}}{\operatorname{raB}_{3}}\right)^{-2} \\
& \text { and } \quad \rho_{p_{3,9}}:=\rho p B_{3} \\
& \operatorname{\rho r}_{\mathrm{pB}_{3, \text { is }}}:=\rho \operatorname{\rho rpB}_{3}, \text { is } \cdot\left(\frac{\mathrm{TpB}_{3}, \mathbf{1}}{\mathrm{TpB}_{3}, \text { is }}\right) \cdot\left(1+\frac{\operatorname{vperpB_{3},\text {is}} \cdot \delta \mathrm{tB}_{3}}{\mathrm{raB}_{3}}\right)^{-2} \\
& \rho \mathbf{p B}_{3,9}:=\operatorname{\rho rpB} B_{3,9}
\end{aligned}
$$

\begin{tabular}{|r|}
\hline 0.65 \\
\hline 0.67 \\
\hline 0.72 \\
\hline 0.82 \\
\hline 0.99 \\
\hline 1.33 \\
\hline 2.11 \\
\hline 5.44 \\
\hline 0 \\
\hline
\end{tabular}

\begin{tabular}{|r|}
\hline 0.13 \\
\hline 0.13 \\
\hline 0.13 \\
\hline 0.13 \\
\hline 0.13 \\
\hline 0.14 \\
\hline 0.14 \\
\hline 0.16 \\
\hline 0 \\
\hline
\end{tabular}

The corrected incident beam energy requirement adjusted for heated ablation plasma loss is

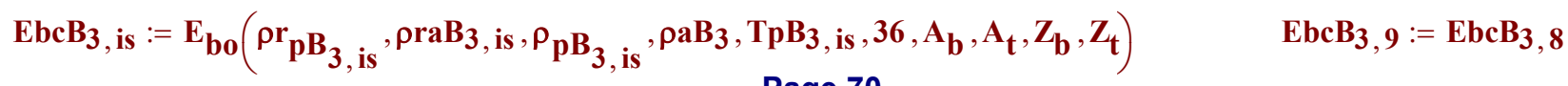




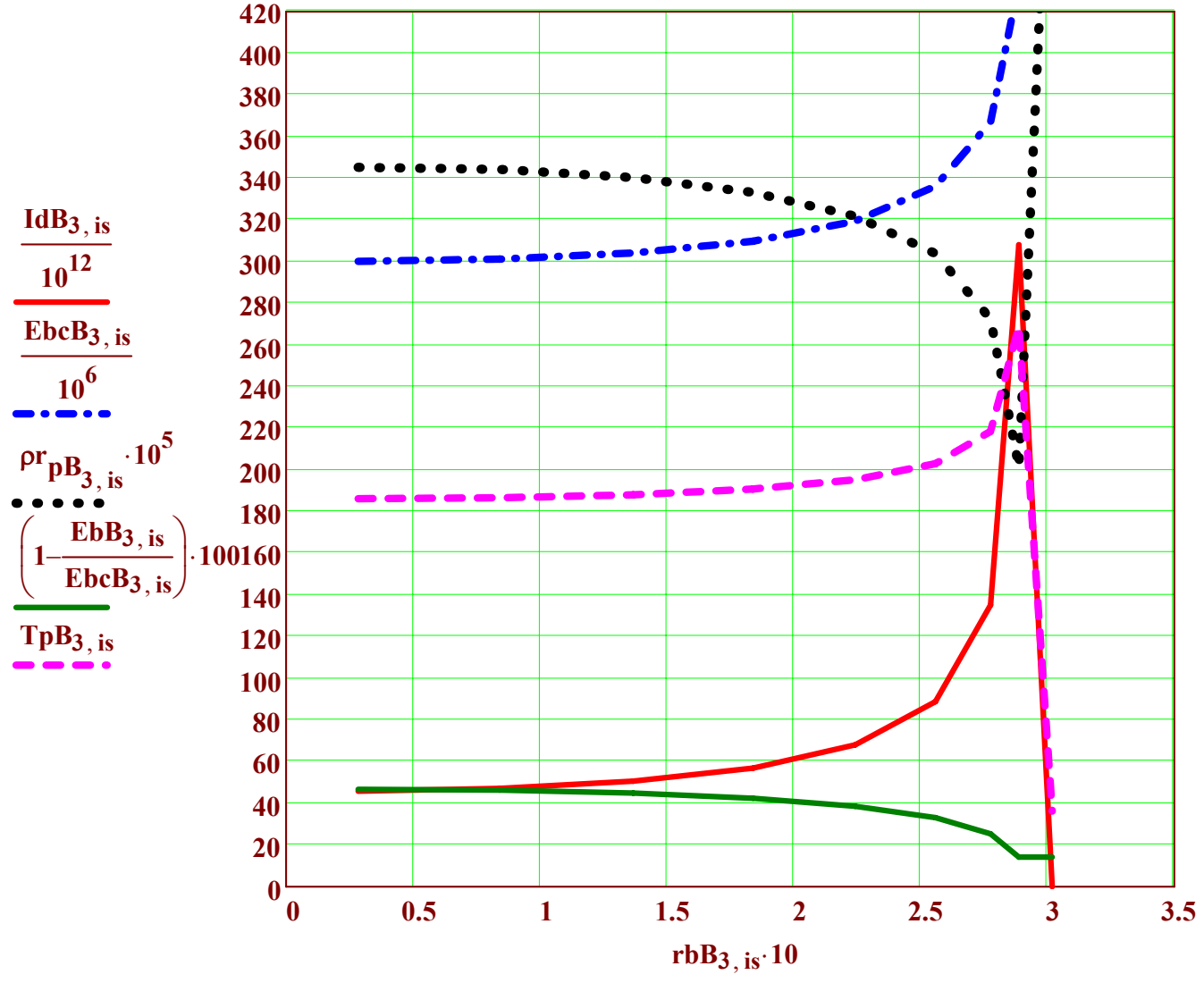

<-Incident Ar beam KE (MeV units)

<-rho-r of ablated plasma $\left(10^{-5} \mathrm{~g} / \mathrm{cm}^{2}\right)$

$<-T_{p}(r)(z-a v e)$ in ablation plasma (units of eV) <-Incident beam intensity $\left(\mathrm{TW} / \mathrm{cm}^{2}\right)$

<- Beam parasitic loss in ablated plasma (percent) <--radius $(\mathrm{mm})$ transverse to polar axis

Figure 42: Plots of polar beam drive intensity (TW/ $\mathrm{cm}^{2}$, one of two sides), incident Ar beam energy (in $\mathrm{MeV}$ units), rho-r of ablated plasma column density $\left(10^{-5} \mathrm{~g} / \mathrm{cm}^{2}\right.$ units) (dotted black line), percent beam loss in ablated plasma, and the temperature $T_{p}$ of the ablation plasma (in $\mathrm{eV}$ units), as functions of radius in the beam channel, transverse to the polar axis near the target, during the third quarter of the ablation drive pulse for the DEMO case $B$.

$$
\begin{aligned}
& \begin{array}{l}
\text { 3rd qtr beam input inc } \\
\text { loss on ablation plasma }
\end{array} \quad \mathrm{EdcB}_{3}:=\sum_{\text {is }=1}^{8}\left[\frac{\mathrm{EbcB}_{3} \text {, is }}{\mathrm{EbB}_{3}, \text { is }} \cdot 2 \cdot\left(\mathrm{WdB}_{3} \cdot \delta \mathrm{VaB}_{3}, \text { is } \cdot \rho \mathrm{aB} 3\right)\right] \quad \mathrm{EdcB}_{3}=1.76 \times 10^{5} \quad \mathrm{~J} \\
& \text { Neglecting beam ablation loss } \quad \mathrm{EdB}_{3}=1.24 \times 10^{5} \quad-->\text { fractional loss }\left(\mathrm{EdcB}_{3}-\mathrm{EdB}_{3}\right) \cdot\left(\mathrm{EdcB}_{3}\right)^{-1}=0.3
\end{aligned}
$$


$\underline{\text { Polar drive parameters for Case } B, 4 \underline{4 h}} \underline{\text { quarter of ablation drive }} \quad \mathrm{tB}_{4}:=\delta \mathrm{tB}_{1}+\delta \mathrm{tB}_{2}+\delta \mathrm{tB}_{3}$

$4^{\text {th }}$ quarter ablator mass $\quad \delta \mathrm{MaB}_{4}=0.003 \quad \mathrm{~g} \quad \mathrm{tB}_{4}=3.93 \times 10^{-8} \quad \mathrm{~s}$, @ density $\quad \rho \mathrm{BB}_{4}:=12 \cdot \rho_{\mathrm{Ho}}$ $4^{\text {th }}$ qtr outer radius $\quad \mathrm{raB3}:=0.25 \quad \mathrm{~cm}$, ablated over time interval $\quad \delta \mathrm{tB}_{4}:=2.4 \cdot 10^{-9} \mathrm{~s}$ $4^{\text {th }}$ qtr inner radius $\quad \mathrm{raB}_{4}:=\left[\mathrm{raB3}^{3}-\left(\frac{3 \cdot \delta \mathrm{MaB}_{4}}{4 \cdot \pi \cdot \mathrm{\rho aB}_{4}}\right)\right]^{0.333} \quad \mathrm{raB}_{4}=0.25 \quad \delta \mathrm{raB}_{4}:=\mathrm{raB}_{3}-\mathrm{raB}_{4}$ $4^{\text {th }}$ qtr layer thickness $\quad \delta \mathrm{raB}_{4}=0.003 \quad \mathrm{~cm}$. Radial KE/exhaust energy efficiency $\eta_{\text {ex }}(1.5,0.2)=0.63$ $4^{\text {th }}$ qtr drive energy $\quad \mathrm{EdB}_{4}:=7.75 \cdot 10^{4} \cdot \eta_{\mathrm{ex}}(1.5,0.2)^{-1} \quad \mathrm{EdB}_{4}=1.24 \times 10^{5} \quad \mathrm{~J}$ Power* $^{*} \quad \operatorname{PdB}_{4}:=\mathrm{EdB}_{4} \cdot\left(\delta \mathrm{tB}_{4}\right)^{-1}$ $\mathrm{PdB}_{4} \cdot 10^{-12}=52 \quad$ TW. $4^{\text {th }}$ qtr energy density $\quad \mathrm{WdB}_{4}:=\mathrm{EdB}_{4} \cdot\left(\delta \mathrm{MaB}_{4}\right)^{-1} \quad \mathrm{WdB}_{4}=3.69 \times 10^{7} \quad \mathrm{~J} / \mathrm{g}$ $4^{\text {th }}$ quarter ablation front pressure $\quad \mathrm{WdB}_{4} \cdot \rho \mathrm{aB}_{4} \cdot 10^{6} \cdot 3^{-1} \cdot 10^{-11}=148 \quad \mathrm{MB} \quad \mathrm{WdB}_{4} \cdot \frac{\mathrm{m}_{\mathrm{h}}}{10 \cdot \mathrm{e}}=39 \quad \mathrm{eV}$ (2) equiv. $4^{\text {th }}$ qtr shell volume $\delta \mathrm{VaB}_{4}$, is $:=2 \cdot \pi \cdot\left(\mathrm{raB}_{4}+\frac{\delta \mathrm{raB}_{4}}{2}\right)^{2} \cdot \delta \mathrm{raB}_{4} \cdot \sin \left(\theta_{\text {is }}+\frac{\pi}{32}\right) \cdot \frac{\pi}{16} \quad \mathrm{~cm}^{3}$ 2. $\sum_{\text {is }=1}^{8} \delta \mathrm{VaB}_{4}$, is $=0.003 \quad \mathrm{~cm}^{3} \quad \frac{4}{3} \cdot \pi \cdot\left[\left(\mathrm{raB}_{4}+\delta \mathrm{raB}_{4}\right)^{3}-\left(\mathrm{raB}_{4}\right)^{3}\right]=0.003 \quad$ Shell volume checks OK! Beam convergence angle $\quad \theta b_{\text {is }}:=0.125 \cdot \sin \left(\theta_{\text {is }}\right) \quad$ Beam-pathlength-4th qtr $\quad \delta z a B_{4}$, is $:=\frac{\delta \text { raB }_{4}}{\cos \left(\theta_{\text {is }}-\theta b_{\text {is }}\right)}$ Beam range $\quad \rho r a B_{4}$, is $:=\rho \mathrm{B}_{4} \cdot \delta \mathrm{zaB}_{4}$, is $\quad$ \& energy* $\quad \mathrm{EbB}_{4}$, is $:=\mathbf{E}_{\mathbf{b f}}\left(\rho \mathrm{B}_{4}, \rho \mathrm{raB} 4\right.$, is $\left., 0.01,39, \mathbf{A}_{\mathbf{b}}, \mathbf{A}_{\mathbf{t}}, \mathbf{Z}_{\mathbf{b}}, \mathbf{Z}_{\mathbf{t}}\right)$ Incident beam radius vs polar angle $\theta \quad \mathrm{rbB}_{4}$, is $:=\operatorname{raB}_{4} \cdot \sin \left(\theta_{\text {is }}+\frac{\pi}{32}\right) \quad \quad \operatorname{rbB}_{4}, 9:=\operatorname{raB} 3+\delta z_{3} B_{4}, 9 \cdot \theta b_{9}$ Beam illumination width (cm) per $\theta$ increment $\quad \delta \mathrm{rbB}_{4}$, is $:=\left(\mathrm{raB}_{4}+\frac{\delta \mathrm{raB}_{4}}{2}\right) \cdot \sin \left(\theta_{\text {is }}+\frac{\pi}{16}\right)-\mathrm{raB}_{4} \cdot \sin \left(\theta_{\text {is }}\right)$ Beam deposition intensity $\left(r_{b}\right) \quad \operatorname{IdB}_{4}$, is := $\mathrm{WdB}_{4} \cdot \delta \mathrm{VaB}_{4}$, is $\cdot \rho \mathrm{BB}_{4} \cdot\left(\delta \mathrm{tB}_{4} \cdot 2 \cdot \pi \cdot \mathrm{rbB}_{4} \text {, is } \delta \operatorname{rbB}_{4} \text {, is }\right)^{-1} \quad \operatorname{IdB}_{4}, 9:=0$ *Beam power, energy before taking beam losses into account

Table 24: 2-D polar drive requirements for Case B, $4^{\text {th }}$ quarter ablation period.

\begin{tabular}{|c|c|c|c|c|c|}
\hline$\theta_{\text {is }}=$ & $\operatorname{rbB}_{4, \text { is }}=$ & $\delta \mathrm{zaB}_{4}$, is $=$ & $\operatorname{\rho raB}_{4, \text { is }}=$ & $\mathrm{IdB}_{4, \text { is }} \cdot 10^{-12}=$ & $\mathrm{EbB}_{4}$, is $\cdot 10^{-6}=$ \\
\hline 0 & 0.02 & 0.003 & 0.0039 & 61 & 185 \\
\hline 0.2 & 0.07 & 0.003 & 0.004 & 63 & 188 \\
\hline 0.39 & 0.12 & 0.003 & 0.0042 & 68 & 194 \\
\hline 0.59 & 0.16 & 0.004 & 0.0045 & 77 & 207 \\
\hline 0.79 & 0.19 & 0.004 & 0.0051 & 92 & 227 \\
\hline 0.98 & 0.22 & 0.005 & 0.0061 & 122 & 260 \\
\hline 1.18 & 0.24 & 0.007 & 0.008 & 189 & 316 \\
\hline 1.37 & 0.25 & 0.01 & 0.0125 & 464 & 428 \\
\hline 1.57 & 0.25 & 0.026 & 0.0314 & 0 & 428 \\
\hline $\begin{array}{l}\text { Polar } \\
\text { angle (rad) }\end{array}$ & $\begin{array}{l}\text { Beam } \\
\text { radius }(\mathrm{cm})\end{array}$ & $\begin{array}{l}\text { Shell } \\
z \text { depth }(\mathrm{cm})\end{array}$ & $\begin{array}{l}\text { Beam } \\
\text { range } \mathrm{g} / \mathrm{cm}^{2}\end{array}$ & $\begin{array}{l}\text { Beam } \\
\text { Intensity } \mathrm{TW} / \mathrm{cm}^{2}\end{array}$ & $\begin{array}{l}\text { Argon Beam Energy } \\
\text { (MeV) (No parasitic } \\
\text { ablation plasma loss) }\end{array}$ \\
\hline
\end{tabular}

Page 58 
Now lets correct for ablation plasma in this ablation period of The fourth quarter ablated plasma mass expands in this time interval to a radius $\mathrm{u}_{\mathrm{aveB} 4}:=2.9 \cdot 10^{7} \quad \operatorname{rpB}_{4}:=\mathrm{raB3}+\left(\mathrm{u}_{\mathrm{ex}}(1.5,0.2)-\mathrm{u}_{\mathrm{aveB} 4}\right) \cdot \delta \mathrm{tB}_{4} \quad \mathrm{rpB}_{4}=0.2 \quad \mathrm{~cm} \quad \mathrm{raB} 4:=\mathrm{raB3}-\mathrm{u}_{\mathrm{aveB} 4} \cdot \delta \mathrm{tB} 4$

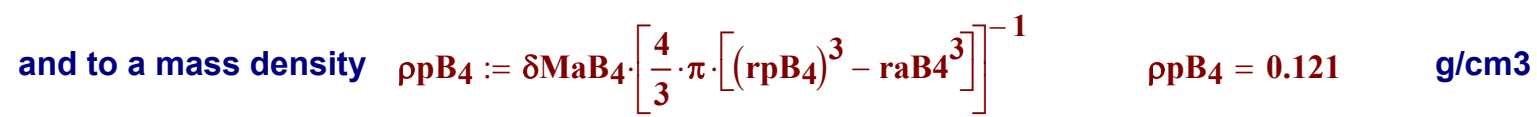
and to a rho-r $\quad \rho r p B_{4}$, is $:=\rho p B_{4} \cdot\left(r p B_{4}-r a B 4\right)+\rho r_{3} B_{3} \quad \rho r p B_{4}, 8=0.008 \quad \mathrm{~g} / \mathrm{cm}^{2}$, now $\sim e q u a l$ to the (Note we add ablation rho-r's accumulated up through the third qtr!) ave $4^{\text {th }}$ qtr ablator range.

Lets make a first order correction to the exhaust plasma temperature, assuming all beam energy deposited into the ablation plasma mass within the beam channel

$$
\Delta \mathbf{E b p B}_{4}, \text { is }:=\left(\mathbf{E}_{\mathbf{b o}}\left(\rho r p B_{4}, \text { is }, \rho r a B_{4}, \text { is }, \rho p B_{4}, \rho B_{4}, 250,39, \mathbf{A}_{\mathbf{b}}, \mathbf{A}_{\mathbf{t}}, \mathbf{Z}_{\mathbf{b}}, \mathbf{Z}_{\mathbf{t}}\right)-\mathbf{E b B}_{4}, \text { is }\right) \cdot\left(\mathbf{E b B}_{4}, \text { is }\right)^{-1}
$$

goes into incremental thermal energy $3 \Delta T_{p}$, and for the moment lets assume the ablated plasma density does not change; then we have, adding the last qtr beam input to heating on top:

$$
\begin{aligned}
& \mathrm{TpB}_{4} \text {, is }:=\frac{\mathrm{IdB}_{4}, \text { is } \cdot \delta \mathrm{tB}_{4} \cdot \Delta \mathrm{EbpB}_{4}, \text { is }}{\rho r p B_{4}, \text { is }} \cdot \frac{\mathrm{m}_{\mathbf{h}}}{6 \cdot \mathrm{e}} \ldots \\
& \mathrm{TpB}_{4, \text { is }}=\frac{\mathrm{TpB}_{4, \text { is }}}{\mathrm{T}_{\mathrm{ex}}(1.5,0.2)}= \\
& +\mathrm{TpB}_{3} \text {, is } \\
& T_{\text {ex }}(1.5,0.2)=36.18
\end{aligned}
$$

\begin{tabular}{|c|c|c|}
\hline & $\operatorname{vperp}_{4}$, is $\cdot \delta \mathrm{tB}_{4}$ & $\operatorname{vperpB}_{4}$, is $\cdot \delta \mathrm{tB}_{4}$ \\
\hline $\operatorname{vperpB}_{4}$, is $=$ & $\delta \mathrm{rbB}_{4}$, is & $\mathbf{r a B}_{4}$ \\
\hline $1.76 \cdot 107$ & 0.87 & 0.17 \\
\hline $1.77 \cdot 107$ & 0.9 & 0.17 \\
\hline $1.78 \cdot 107$ & 0.98 & 0.17 \\
\hline $1.8 \cdot 107$ & 1.12 & 0.18 \\
\hline $1.84 \cdot 107$ & 1.38 & 0.18 \\
\hline $1.89 \cdot 107$ & 1.87 & 0.18 \\
\hline $2 \cdot 107$ & 3.07 & 0.19 \\
\hline $2.34 \cdot 107$ & 8.82 & 0.23 \\
\hline 0 & 0 & 0 \\
\hline
\end{tabular}

\begin{tabular}{|r|}
\hline 231 \\
\hline 232 \\
\hline 235 \\
\hline 240 \\
\hline 248 \\
\hline 261 \\
\hline 287 \\
\hline 379 \\
\hline 36 \\
\hline
\end{tabular}

\begin{tabular}{|r|}
\hline 1.699 \\
\hline 1.673 \\
\hline 1.594 \\
\hline 1.463 \\
\hline 1.282 \\
\hline 1.054 \\
\hline 0.782 \\
\hline 0.47 \\
\hline 1.273 \\
\hline
\end{tabular}

\begin{tabular}{|r|}
\hline $1.85 \cdot 10^{8}$ \\
\hline $1.88 \cdot 108$ \\
\hline $1.94 \cdot 108$ \\
\hline $2.07 \cdot 108$ \\
\hline $2.27 \cdot 108$ \\
\hline $2.6 \cdot 108$ \\
\hline $3.16 \cdot 108$ \\
\hline $4.28 \cdot 108$ \\
\hline $4.28 \cdot 10^{8} 8$ \\
\hline
\end{tabular}

One can see in this table that the ablation plasma is substantially heated (Te increases 5-8X) (most in this 4th qtr of the pulse). Check if heating (pressurization) can still cause significant enhanced expansion in the shorter transverse to the beam channel (polar axis) direction in the shorter time $\delta$ tB $_{4}$ :

$$
\begin{aligned}
& \operatorname{vperpB}_{4} \text { is }:=10^{2} \cdot \sqrt{\frac{5 \cdot\left[\left(\mathrm{TpB}_{4}, \text { is }\right)-\mathrm{T}_{\mathrm{ex}}(1.5,0.2)\right] \cdot \mathrm{e}}{3 \cdot \mathrm{m}_{\mathrm{h}} \cdot 10^{-3}}} \\
& \rho_{p_{4, \text { is }}}:=\rho p B_{4} \cdot\left(\frac{T p B_{4,1}}{T_{p B} B_{4, \text { is }}}\right) \cdot\left(1+\frac{\text { vperpB } 4, \text { is } \cdot \delta B_{4}}{\operatorname{raB}_{4}}\right)^{-2} \\
& \text { and } \quad \rho_{\mathrm{pB}_{4,9}}:=\rho \mathrm{pB}_{4} \\
& \rho \mathrm{pB}_{4, \text { is }}:=\rho \mathrm{\rho pB}_{4}, \text { is } \cdot\left(\frac{\mathrm{TpB} 4,1}{\mathrm{TpB}_{4, \text { is }}}\right) \cdot\left(1+\frac{\mathrm{vperpB}_{4}, \text { is } \cdot \delta \mathrm{tB}_{4}}{\mathrm{raB}_{4}}\right)^{-2} \\
& \rho \mathbf{p B}_{4,9}:=\rho \operatorname{prpB}_{4,9}
\end{aligned}
$$

The corrected incident beam energy requirement adjusted for heated ablation plasma loss is

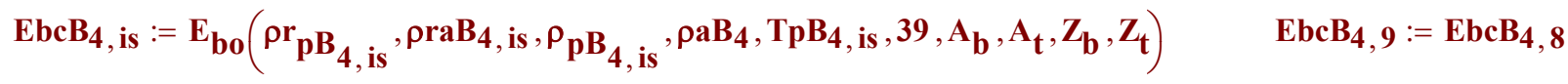




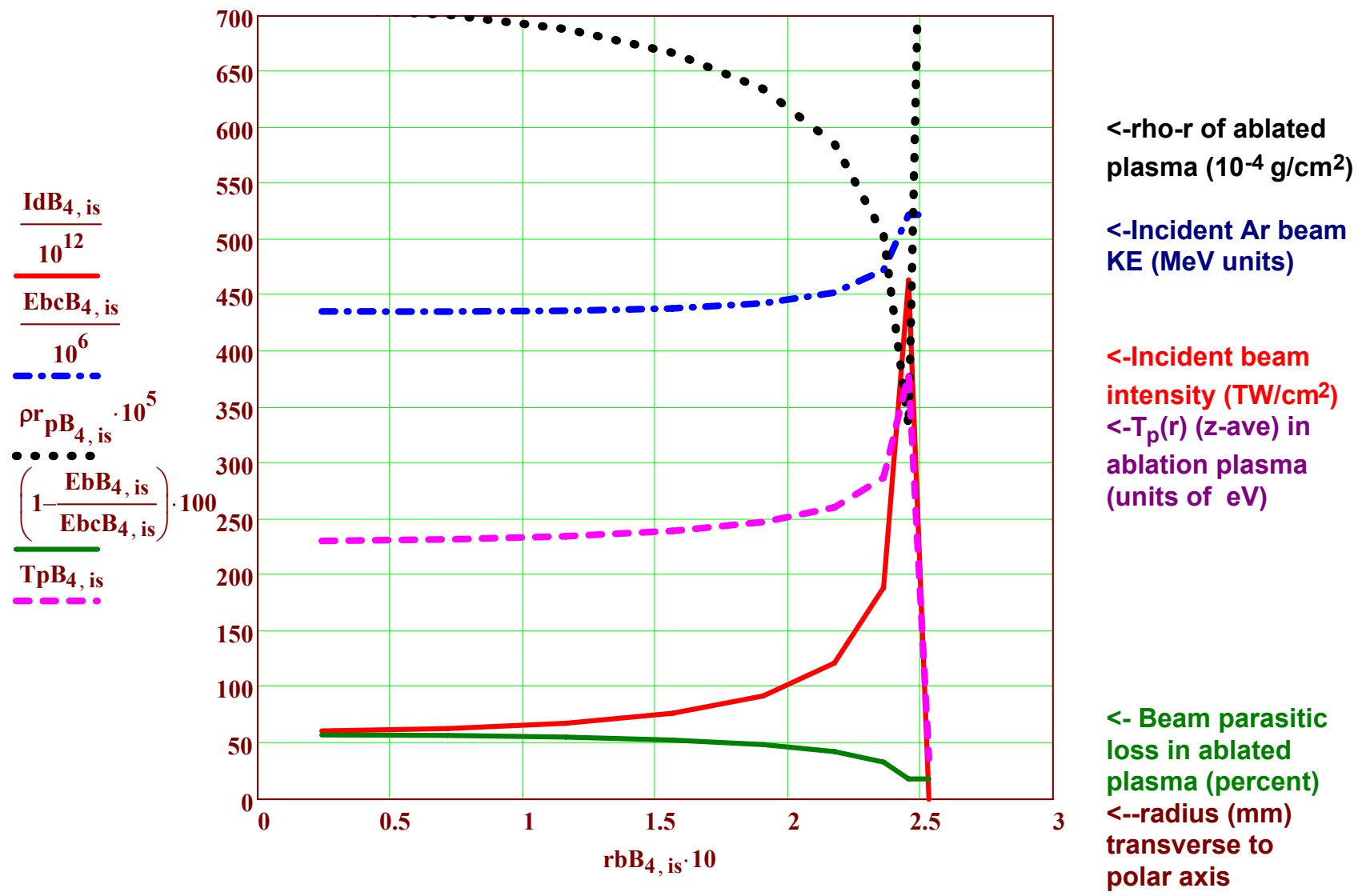

Figure 43: Plots of polar beam drive intensity (TW/ $\mathrm{cm}^{2}$, one of two sides), incident Ar beam energy (in MeV units), rho-r of ablated plasma column density $\left(10^{-5} \mathrm{~g} / \mathrm{cm}^{2}\right.$ units) (dotted black line), percent beam loss in ablated plasma, and the temperature $\mathrm{T}_{\mathrm{p}}$ of the ablation plasma (in $\mathrm{eV}$ units), as functions of radius in the beam channel, transverse to the polar axis near the target, during the fourth quarter of the ablation drive pulse for the small DEMO case $A$.

$4^{\text {th }}$ qtr beam input inc loss on ablation plasma

$$
\mathrm{EdcB}_{4}:=\sum_{\text {is }=1}^{8}\left[\frac{\mathrm{EbcB}_{4}, \text { is }}{\mathrm{EbB}_{4}, \text { is }} \cdot 2 \cdot\left(\mathrm{WdB}_{4} \cdot \delta \mathrm{VaB}_{4}, \text { is } \cdot \mathrm{\rho aB}_{4}\right)\right]
$$$$
\mathrm{EdB}_{4}=1.24 \times 10^{5}
$$
-->fractional loss

Neglecting beam ablation loss

$$
\text { EdriveB }_{\text {tot }}:=\sum_{\mathrm{js}=1}^{4} \text { EdcB }_{\mathrm{js}}
$$

Overall coupling efficiency

$$
\eta_{\text {dfBc }}:=\frac{E_{\text {fuelB }}}{\text { Edrive }_{\text {tot }}} \quad \eta_{\text {dfBc }}=0.303
$$

$\mathrm{EdcB}_{4}=1.98 \times 10^{5} \quad \mathrm{~J}$

$\left(\mathrm{EdcB}_{4}-\mathrm{EdB}_{4}\right) \cdot\left(\mathrm{EdcB}_{4}\right)^{-1}=\mathbf{0 . 3 7}$

Compressed fuel energy

$$
\mathrm{E}_{\text {fuelB }}:=\mathbf{2 \cdot 1 0 ^ { 5 }}
$$

(a record > three times laser direct drive (if it holds up)

This increased overall coupling efficiency of $30 \%$ for a complete modelling of 2-D beam interaction with 2-D ablation plasma expansion, compares to $24 \%$ taking 2-D effects only in expansion of ablation plasma (page 35). The highest ablation plasma temperatures $(350 \mathrm{eV})$ late in the drive pulse for the large targets (Case B) (Fig 43 above) is just high enough to cause a slight beam range increase in the $450 \mathrm{MeV} \mathrm{Ar}$ beams (in view of Fig. 33), so all of the improvements in coupling efficiency in 2-D is due to beam geometry and plasma expansion alone. However, $M_{0} / M_{f}=2$ leads to more range lengthening effect (next). 


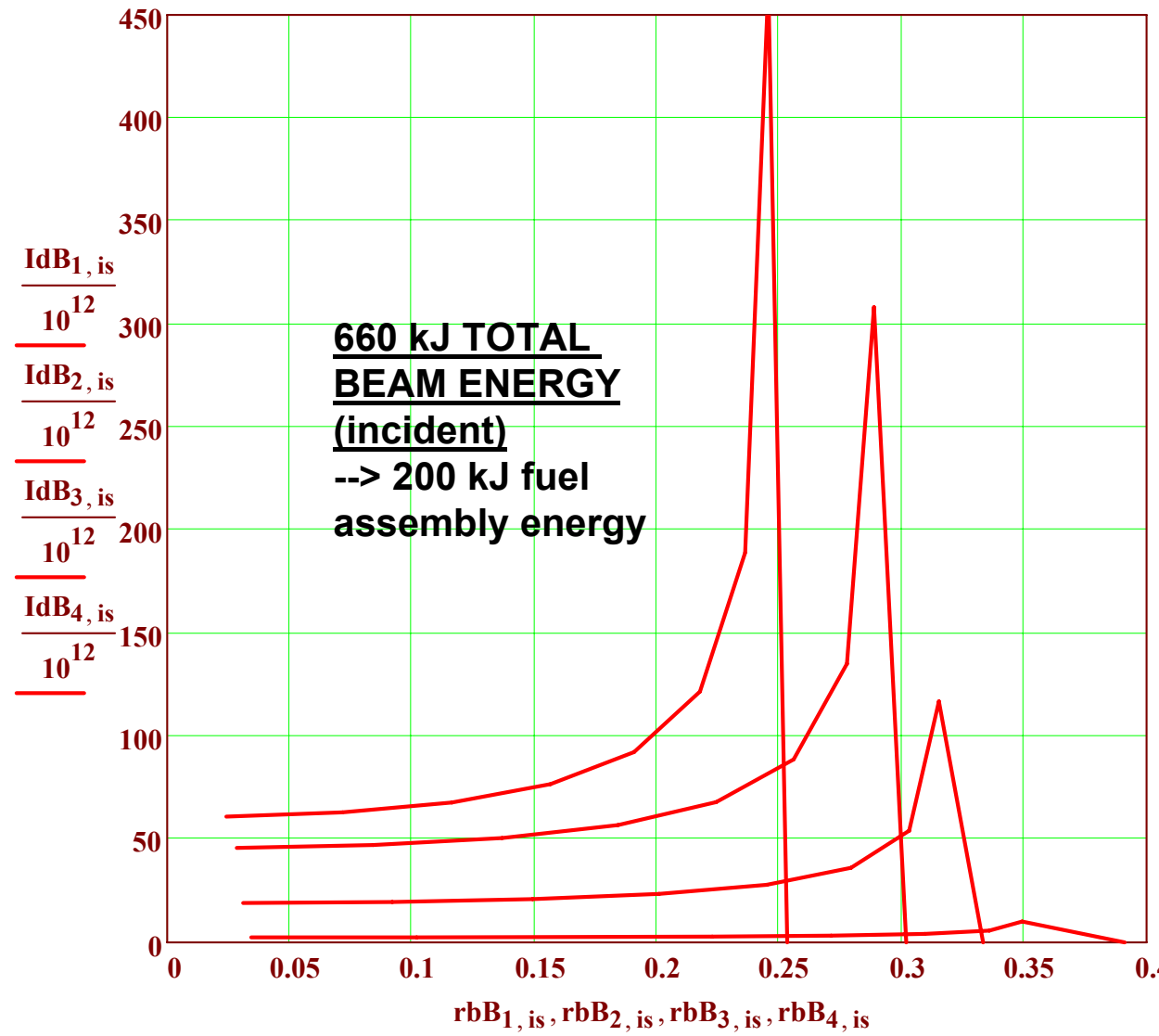

Beam intensity (one of two sides)

(TW/cm²)

$<-@ t=39$ ns

$<-@ \mathrm{t}=37$ ns

$<-@ \mathrm{t}=32$ ns

$<-@ \mathrm{t}=0$

$<--$ beam radius at the target

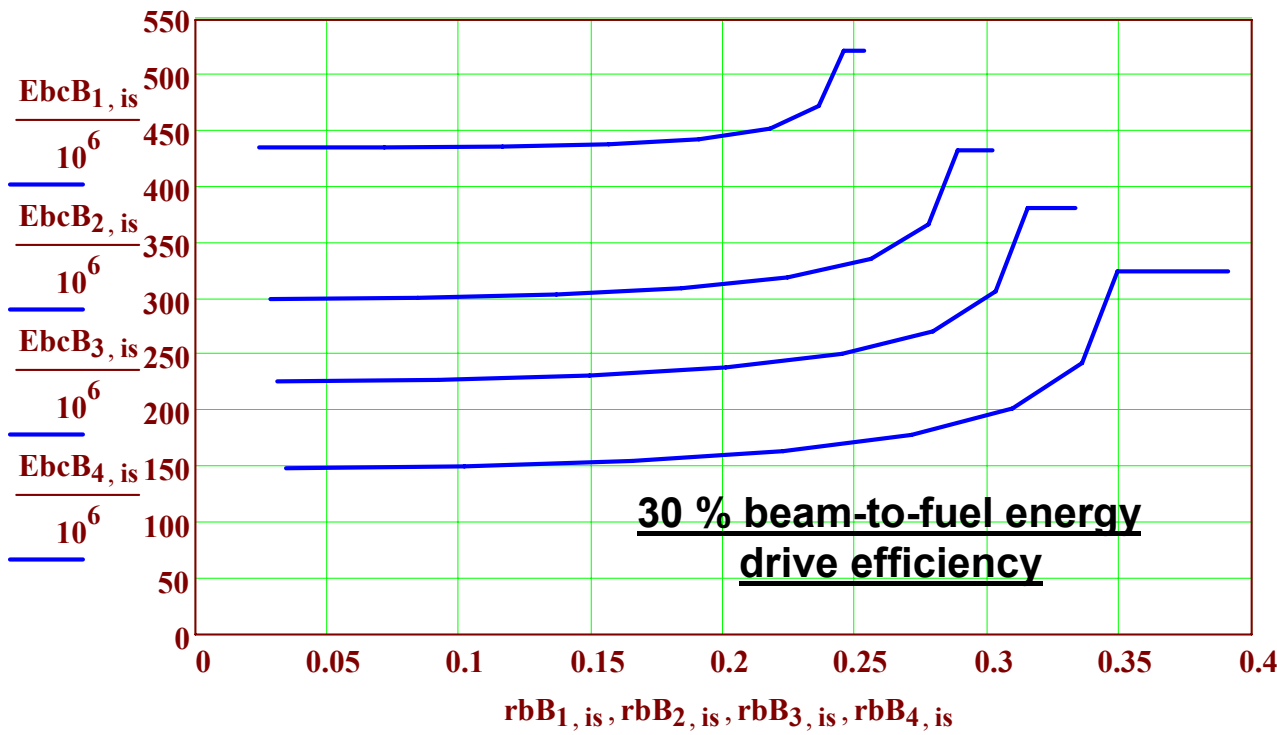

Incident beam ion energy ( $\mathrm{MeV}$ ) $<-@ \mathrm{t}=39$ ns

$<-@ \mathrm{t}=37$ ns

$<-@ \mathrm{t}=32 \mathrm{~ns}$

$<-@ \mathbf{t}=0$

<--beam radius at the target

Figure 44. Beam intensity profiles (TW/cm2) (one of two sides) (red curves-top) and beam ion energies for Argon (in MeV) (blue curves-bottom) vs radius for the small Case B DEMO target, required for symmetric polar (two sided) drive with spherically symmetric ablation, at four times during the implosion when the $\mathrm{H} 2$ ablator mass is reduced by $1 / 4$. Beam losses in ablated plasma are accounted for in the 2-D model including density reductions due to heating Tp and "hole-boring" effects. Note that local reduction of abaltion plasma column density in the high intensity beam rim tends to reduce the variation in ion energy (range) between the polar axis and the rim. Future work will seek use of P2 variations of ablator thickness $\Delta$ ra( $(\theta)$ [shims] to enable time-averaged symmetry using beam profiles with less peaked rims. 


\section{Range lengthening estimate for a lower ablator mass DEMO case.}

Estimated beam range lengthening effects for the a small DEMO $(E f=200 \mathrm{~kJ})$ case, but with less ablator mass $M_{o} / M_{f}=2$ (more laser like case for the small DEMO, and more like John Perkins example, except for hydrogen ablator.). Use same beam-ablation plasma interaction model model as in $M_{0} / M_{f}=5$. First note that there is four times less ablator mass for the same payload mass:

$$
\delta \mathrm{MaBc}:=\frac{\delta \mathrm{MaB}_{4}}{4} \quad \delta \mathrm{MaBc}=8.375 \times 10^{-4} \quad \mathrm{~g} \text {, and so there will also be }
$$

one fourth of the ablator shell range for the ion beams as before, still assuming the ion range is a quarter of the initial total ablator mass. Thus the indicent ion beam energy will be reduced to get the required $1 / 4$ ablator range. For the same payload mass and velocity (same implosion time), and same ablator $\mathrm{H} 2$ mass density at each stage, a comparison of Case $A$ for $M_{o} / M_{f}=5$ versus Case $C$ for $M_{\mathrm{o}} / \mathrm{M}_{\mathrm{f}}=2$ shows that the required incident ion beam intensity is three times higher, the shell radii are 0.81 times less, while the implosion time is half as long. Thus, roughly, we find:

$$
\mathrm{IdBc}_{4} \text {, is := 3. IdB } 4 \text {, is } \quad \operatorname{IdBc}_{4}, 9:=0 \quad \delta \mathrm{tBc}_{4}:=0.5 \cdot \delta \mathrm{tB}_{4} \quad \mathrm{rbBc}_{4}, \text { is }:=0.81 \cdot \mathrm{rbB}_{4} \text {, is }
$$

Beam range $\rho \mathrm{raBc}_{4}$, is $:=\rho \mathrm{BB}_{4} \cdot 0.25 \cdot \delta \mathrm{zaB}_{4}$, is

Energy (not corrected $\quad \mathbf{E b B c}_{4}$, is $:=\mathbf{E}_{\mathbf{b f}}\left(\rho \mathrm{BB}_{4}, \rho \mathrm{raBc} 4\right.$, is $\left., 0.01,100, \mathbf{A}_{\mathbf{b}}, \mathbf{A}_{\mathbf{t}}, \mathbf{Z}_{\mathbf{b}}, \mathbf{Z}_{\mathbf{t}}\right) \quad \mathbf{E b B c}_{4,9}:=\mathbf{E b B c}_{4,8}$ yet for ablation plasma

Now lets correct for ablation plasma at the $\ln 5 / \ln 2$ faster exhaust velocity

The fourth quarter ablated plasma mass expands in this time interval to a radius

$$
\frac{\delta \mathrm{tBc}_{4}}{10^{-9}}=1.2 \quad \mathrm{~ns}
$$

$$
\begin{aligned}
& \mathrm{u}_{\mathrm{anaBA}}:=2.9 \cdot 10^{7} \quad \operatorname{rpBc}_{4}:=\operatorname{raB}_{4}+\left(\mathrm{u}_{\mathrm{ex}}(1.5,0.2) \cdot \frac{\ln (5)}{\ln (2)}-\mathrm{u}_{\mathrm{aveB} 4}\right) \cdot \delta \mathrm{tBc}_{4} \quad \operatorname{rpBc}_{4}=0.28 \quad \mathrm{~cm} \\
& \operatorname{raBc} 4:=0.81 \cdot \operatorname{raB3}-\mathrm{u}_{\mathrm{aveB} 4} \cdot \delta \mathrm{tBc}_{4} \quad \operatorname{raBc} 4=0.17
\end{aligned}
$$

and to a mass density $\quad \operatorname{pBBc}_{4}:=\delta \mathrm{MaBc} \cdot\left[\frac{4}{3} \cdot \pi \cdot\left[\left(\operatorname{rpBc}_{4}\right)^{3}-\mathrm{raBc}^{3}\right]\right]^{-1} \quad \rho \mathrm{pBc}_{4}=0.0124 \quad \mathrm{~g} / \mathrm{cm} 3$

and to rho-r $\quad \rho \mathrm{pBc}_{4}$, is $:=\rho \mathrm{pBc}_{4} \cdot\left(\mathrm{rpBc}_{4}-\mathrm{raB} 4\right)+0.25 \cdot \rho \mathrm{pB}_{3}$, is

$$
\operatorname{\rho rpBc}_{4}, 8=0.0017 \quad \mathrm{~g} / \mathrm{cm}^{2}, \sim \text { equal to the } M_{\mathrm{o}} / \mathrm{M}_{\mathrm{f}}=2 \quad 4^{\text {th }} \text { qtr ablator. }
$$

Lets make a first order correction to the exhaust plasma temperature, assuming all beam energy deposited into the ablation plasma mass within the beam channel

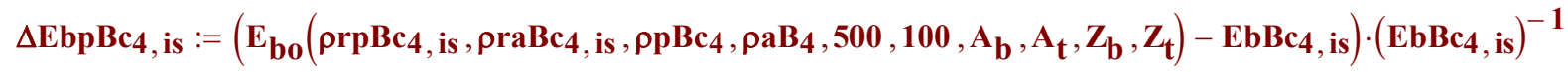

goes into incremental thermal energy $3 \Delta T_{p}$, and for the moment lets assume the ablated plasma density does not change; then we have, adding the last qtr beam input to heating on top:

$$
\begin{aligned}
\mathrm{TpBc}_{4}, \text { is }:= & \frac{\mathrm{IdBc}_{4}, \text { is } \cdot \delta \mathrm{tBc}_{4} \cdot \Delta \mathrm{EbpBc}_{4}, \text { is }}{\operatorname{\rho rpBc}_{4}, \text { is }} \cdot \frac{\mathrm{m}_{\mathrm{h}}}{6 \cdot \mathrm{e}} \ldots \\
& +3 \cdot \mathrm{TpB}_{3}, \text { is }
\end{aligned}
$$

Table 25 Ablation plasma for $M_{o} / M_{f}=2$ small DEMO

Note that Tp now with much higher intensities with lower beam range for the $M_{o} / M_{f}=2$ lower ablator mass DEMO Case $B$ *is* now high enough for a substantial beam range increase as ve is now > vbeam! (see Fig. 33). Note these dimensions, masses, beam energies and times are roughly comparable to John Perkins $1 \mathrm{MJ}$ example! 

Corrected exhaust temperature for the Mo/Mf=2 case $\quad$ Texc $:=T_{\text {ex }}(1.5,0.2) \cdot\left(\frac{\ln (5)}{\ln (2)}\right)^{2} \quad$ Texc $=195$
\[ \operatorname{TpBc}_{4,9}:=1.01 \cdot \mathrm{Texc} \]

Check if heating (pressurization) can still cause significant enhanced expansion in the shorter transverse to the beam channel (polar axis) direction in the shorter time $\delta \mathrm{tBc}_{4}$ :

$\operatorname{vperpBc}_{4}$, is $:=10^{2} \cdot \sqrt{\frac{5 \cdot\left(\mathrm{TpBc}_{4}, \text { is }-\mathrm{Texc}\right) \cdot \mathrm{e}}{3 \cdot \mathrm{m}_{\mathrm{h}} \cdot 10^{-3}}} \mathrm{~cm} / \mathrm{s}$

Table 26: Ablation plasma expansion parameters for the small $M_{o} / M_{f}=2 D E M O$ case ( Perkins 1 MJ case)

We see in Table 26 that the $\sim 2 \mathrm{x}$ higher exhaust velocites are offest by the shorter ablation times for $M_{o} / M_{f}=2$, such that ablation expansion ratios are about the same as in $M_{o} / M_{f}=5$, even though the plasma Tp and thermal speed is much higher.

\begin{tabular}{|c|c|c|}
\hline & $\operatorname{vperpB}_{4}$, is $\cdot \delta \mathrm{tBc}_{4}$ & $\operatorname{vperpBc}_{4}$, is $\cdot \delta \mathrm{tBc}_{4}$ \\
\hline $\operatorname{vperpBc}_{4}$, is $=$ & raBc4 & $0.81 \cdot \delta \mathrm{rbB}_{4}$, is \\
\hline $4.42 \cdot 107$ & 0.13 & 1.35 \\
\hline $4.39 \cdot 107$ & 0.13 & 1.39 \\
\hline $4.29 \cdot 10^{7}$ & 0.13 & 1.46 \\
\hline $4.2 \cdot 107$ & 0.13 & 1.61 \\
\hline $4.14 \cdot 107$ & 0.13 & 1.92 \\
\hline $4.17 \cdot 107$ & 0.14 & 2.54 \\
\hline $4.35 \cdot 10^{7}$ & 0.14 & 4.12 \\
\hline $5.15 \cdot 107$ & 0.17 & 11.98 \\
\hline \begin{tabular}{|c|}
$1.76 \cdot 106$ \\
\end{tabular} & 0 & -0.83 \\
\hline
\end{tabular}

Now finish correcting for the rho's and rho-r's:

$$
\begin{aligned}
& \rho_{\mathrm{pBc}_{4, \text { is }}}:=\rho \mathrm{pBc}_{4} \cdot\left(\frac{\mathrm{TpBc}_{4}, 1}{\mathrm{TpBc}_{4}, \text { is }}\right) \cdot\left(1+\frac{\operatorname{vperpBc}_{4}, \text { is } \cdot \delta \mathrm{tBc}_{4}}{\mathrm{raBc} 4}\right)^{-2} \\
& \text { and } \quad \rho_{\mathrm{pBc}_{4,9}}:=\rho \mathrm{pBc}_{4} \\
& \rho \mathrm{pBc}_{4, \text { is }}:=\rho \operatorname{prpB}_{4}, \text { is } \cdot\left(\frac{\operatorname{TpBc}_{4}, 1}{\operatorname{TpBc}_{4}, \text { is }}\right) \cdot\left(1+\frac{\operatorname{vperpBc} 4, \text { is } \delta \mathrm{tBc}_{4}}{\operatorname{raBc} 4}\right)^{-2} \\
& \rho \mathrm{pBc}_{4,9}:=\rho \mathrm{pBBc}_{4,9}
\end{aligned}
$$

The corrected incident beam energy requirement adjusted for heated ablation plasma loss is

$$
\mathrm{EbcBc}_{4} \text {, is }:=\mathbf{E}_{\mathbf{b o}}\left(\rho \mathrm{pBc}_{4, \text { is }}, \rho \operatorname{prBc}_{4}, \text { is }, \rho_{\mathrm{pBc}_{4}, \text { is }}, \rho \mathrm{BB}_{4}, \mathrm{TpBc}_{4}, \text { is }, 100, \mathrm{~A}_{\mathbf{b}}, \mathbf{A}_{\mathbf{t}}, \mathbf{Z}_{\mathbf{b}}, \mathbf{Z}_{\mathbf{t}}\right)
$$




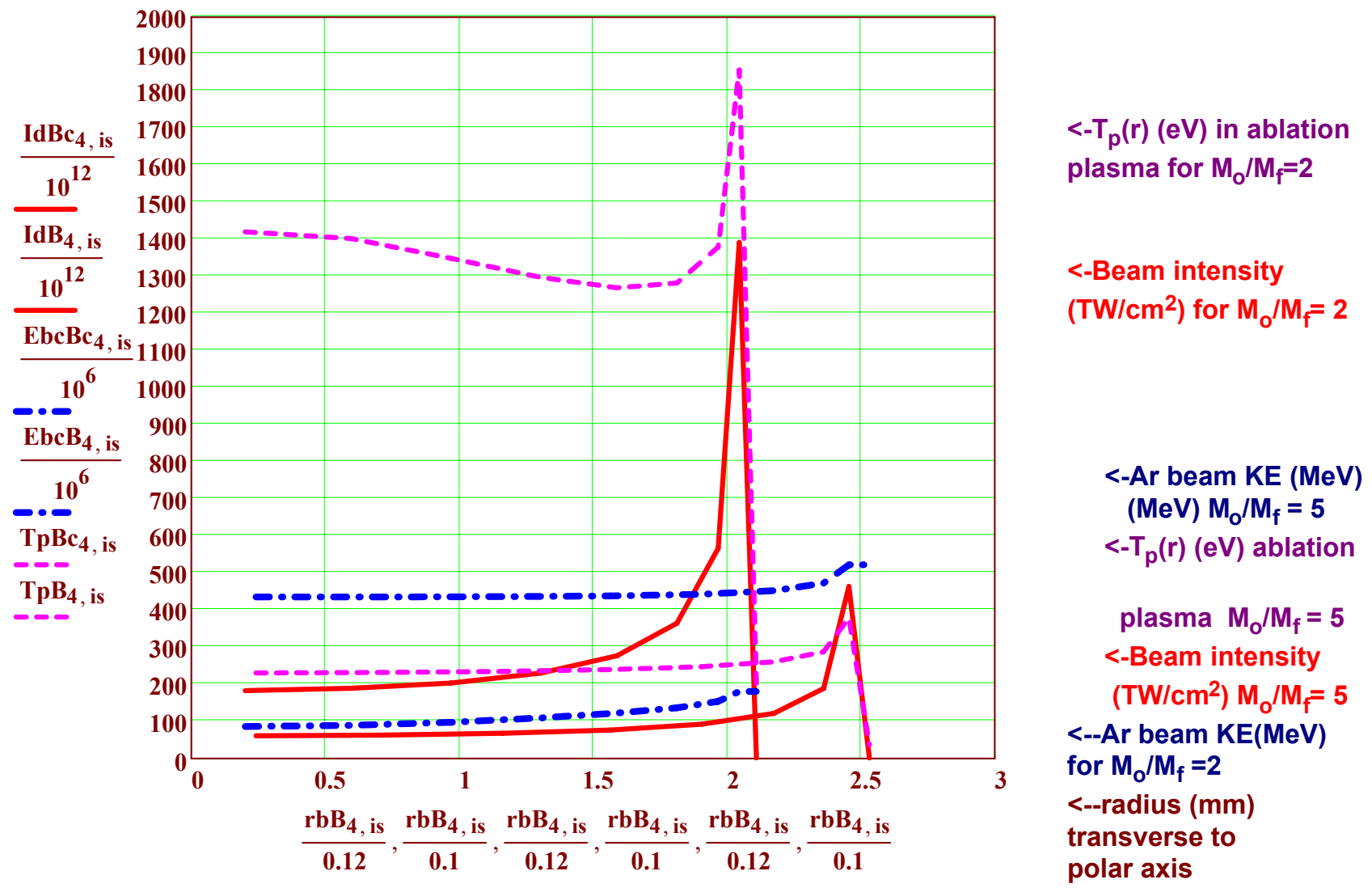

Figure 45: Comparison of polar beam drive intensity (TW/ $\mathrm{cm}^{2}$, one of two sides), incident Ar beam energy (in MeV units), , and the temperature $\mathrm{T}_{\mathrm{p}}$ of the ablation plasma (in $\mathrm{eV}$ units), as functions of radius in the beam channel, transverse to the polar axis near the target, during the fourth quarter of the ablation drive pulse for the small DEMO case $A$, for $M_{o} / M_{f}=2$ out to radius $2.15 \mathrm{~mm}$, compared to previous DEMO case (Fig. 43) with more $H 2$ ablator mass for $M_{o} / M_{f}=5$ out to the larger radius of $2.55 \mathrm{~mm}$.

$$
\text { iso }:=1 \text {.. } 8
$$

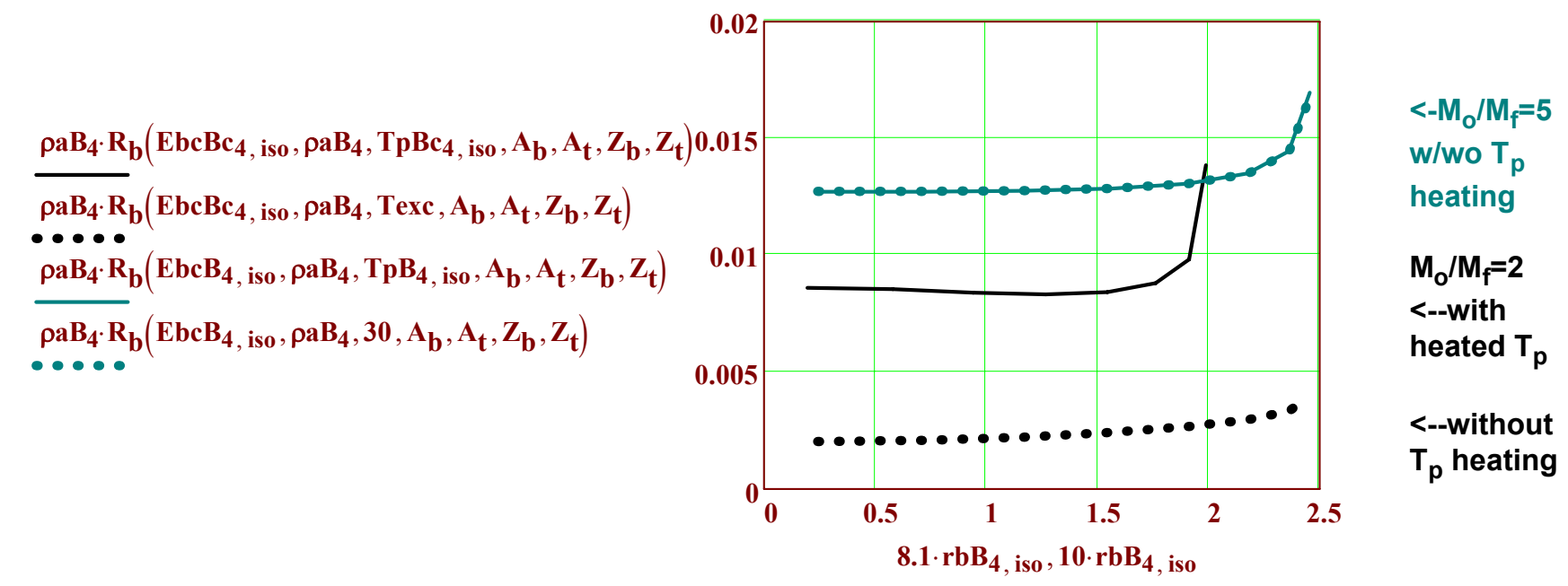

Figure 46: For the lower ablator mass case with $M_{o} / M_{f}=2$, closest to John Perkins 1 MJ example, beam heating vethermal > vbeam increases ion range sufficient to penetrate the lower density ablation cloud layers. John uses a fixed ion energy of $50 \mathrm{MeV}$ Argon, which has even more pronounced effect on Te ("bleaching" like increase of ion range through ablation cloud heating ). 
Estimating incident beam perveance, assuming no plasma neutralization, for the total incident drive beam current for Case A and Case B versus implosion time

$$
\begin{aligned}
& \text { Ibcur }_{\text {tot }_{\mathbf{j s}}}:=\sum_{\mathbf{i s}=1}^{8}\left(\frac{\mathrm{IdA}_{\mathbf{j s}, \text { is }}}{\mathbf{E b c A}_{\mathbf{j s}, \text { is }}} \cdot 2 \cdot \pi \cdot \mathbf{r b A _ { \mathbf { j s } } , \text { is }} \cdot \delta \mathrm{rbA}_{\mathbf{j s}, \text { is }}\right) \\
& \text { Ibcur }_{\text {totB }_{\mathbf{j s}}}:=\sum_{\mathrm{is}=1}^{8}\left(\frac{\mathrm{IdB}_{\mathbf{j s}, \text { is }}}{\mathrm{EbcB}_{\mathbf{j s}, \text { is }}} \cdot 2 \cdot \pi \cdot \mathrm{rbB}_{\mathrm{js}, \text { is }} \cdot \delta \mathrm{rbB}_{\mathrm{js}, \text { is }}\right)
\end{aligned}
$$

$$
\text { man }:=3.1 \cdot 10^{7}
$$

(Amps) -constant in beam perveance)

Incident beam perveance if there was no plasma neutralization of beam space charge and if the beam charge state was $\quad q:=1$

$$
\begin{aligned}
& \mathbf{K}_{\mathbf{b}}(\mathbf{T}, \mathbf{A}, \mathbf{I}, \mathbf{q}):=\frac{2 \cdot \mathbf{q} \cdot \mathbf{I}}{\mathbf{A} \cdot \mathbf{I}_{\mathbf{0}} \cdot(\beta(\mathbf{T}, \mathbf{A}) \cdot \gamma(\mathbf{T}, \mathbf{A}))^{3}} \\
& \mathbf{K}_{\mathbf{b A}}:=\mathbf{K}_{\mathbf{b} \mathbf{b}}\left(\frac{\mathbf{E b c A}_{\mathbf{j s}, 7}}{\mathbf{q}}, \mathbf{A}_{\mathbf{b}}, \mathbf{I b c u r}_{\text {tot }_{\mathbf{j s}}}, \mathbf{q}\right) \quad \mathbf{K}_{\mathbf{b B}}:=\mathbf{K}_{\mathbf{b} \mathbf{b}}\left(\frac{\mathbf{E b c B}_{\mathbf{j s}, 7}}{\mathbf{q}}, \mathbf{A}_{\mathbf{b}}, \text { Ibcur }_{\text {tot }} \mathbf{B}_{\mathbf{j s}}, \mathbf{q}\right)
\end{aligned}
$$

Table 27: Incident beam vacuum perveances for Case A power plant and Case B DEMO examples (assuming there were no local plasma to neutralize the beam space charge).

Case A 3.3 MJ target example

$$
\begin{array}{|r|}
\text { Ibcur }_{\text {tot }} \boldsymbol{A} \\
\hline 1.2 \cdot 104 \\
\hline 6 \cdot 10^{4} \\
\hline 9.5 \cdot 10^{4} \\
\hline 6.8 \cdot 10^{4} \\
\hline
\end{array}
$$

Amperes

$$
\begin{array}{|c|}
\mathbf{K}_{\mathbf{b A}} \mathbf{A}_{\mathbf{j s}} \\
\hline 0.007 \\
\hline 0.024 \\
\hline 0.028 \\
\hline 0.013 \\
\hline
\end{array}
$$

Volts

Case B 660 kJ DEMO target example

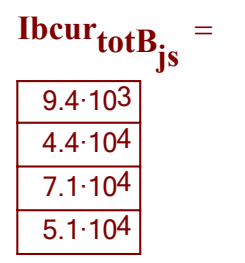

Amperes

\begin{tabular}{|c|} 
EbcB $_{\mathbf{j s}, \mathbf{7}}=$ \\
\begin{tabular}{|c|}
\hline $2.43 \cdot 108$ \\
\hline $3.07 \cdot 108$ \\
\hline $3.67 \cdot 108$ \\
\hline $4.73 \cdot 108$ \\
\hline
\end{tabular}
\end{tabular}

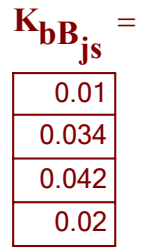

Comment: vaccum perveace is a measure of how much $\mathrm{Z}$ distance (in beam diameter units) it takes for uncompensated beam space charge to double an iniital beam size if propagating balistically in vacuum. Typically in past heavy ion beam fusion studies, a maximum value of vacuum beam perveance of $10^{-4}$ was required to focus beams at all, and $10^{-5}$ to $10^{-6}$ was generally necessary to get the minimum focal spots sizes set by beam emittance (micro-divergence). The values in Table 27 are too high for vacuum focusing by more than two to three orders of magnitude, as a result of constraining the ranges for ablative direct drive. Since beam currents for fixed range and power scale as $A^{-1}$ (and thus $K \sim A^{-2}$ ), one could reduce $K$ by a factor of 10 using Xenon at the expense of higher linac voltage, length and cost, for example, but doing so would still not enable vacuum focusing. Thus, neutralized beam compression and focusing within pre-established background plasma, such as has been demonstrated in the NDCX experiment is a requirement for ablative direct drive HIF. A benefit of using such a technique with ablative direct drive is much lower linac accelerator volyage, length, and cost, and the velocity chirp used in NDCX to longitudinally compress the ion pulses in plasma give rise to a natryally increasing ion energy incident on the target, which is synergistic with the requirment we find for increasing the ion range during the imploasion to minimise parasistic beam losses through the ablation plasma corona. 


\section{Summary of main points learned so far:}

1. This findings of this analytic work and first Lasnex results by John Perkins (LLNL, June 2007) indicate a potential for very high beam-to-compressed-fuel-energy coupling efficiencies (15 to $30 \%)$ using heavy ions in the ablative rocket regime, higher than any hohlraum or laser IFE direct drive Like fast ignition, much more detailed work is required before this ion direct drive potential can be counted on, but results so far are sufficient to justify and guide further detailed 2-D implosion calculations needed to benchmark and refine the physics that is unique to heavy-ion coupling in ablative direct drive implosions.

2. There is likely to be a good practical middle ground between opposing constraints on ion range: the ion range cannot be too large with respect to the ablator thickness, or ability to shape drive pulses for low adiabat implosions would be lost, and the ion range cannot be too small compared to the ablator thickness, lest ablation plasma clouds late in the pulse absorb too much of the incident beam energy. A beam range $=1 / 4$ of the initial ablator thickness, with the ion energy increasing a factor or 2 during the implosion, appears to support good performance, but more work is needed to find the optimum.

3. This analysis indicates that neutralized beam drift compression and final focusing in neutralizing background plasma such as employed in NDCX will be required for ablative direct drive HIF. because of neutralization, the velocity chirp used to compress the beam pulse must be compensated with active time dependant upstream transverse beam modulations on the $100 \mathrm{~ns}$ time scale, to compensate for the beam chromatic focusing errors. Such a technique is planned to be tested in NDCX by 2009. By employing such time-dependent corrections, one also gains the ability to zoom the focus on target with time, which is synergistic with the zooming implied in the two-sided direct drive geometry analyzed here.

4. Two-D Beam requirements on intensity $I_{b}(\theta)$ and ion energy $E b(\theta)$ derived for symmetric implosions with desired two-sided polar drive geometry are calculated to give even higher coupling efficiencies compared to spherically symmetric beam drive because of local reductions in ablated plasma density in the beam channels (a 'hole-boring" effect which reduces parasitic beam loss on ablation plasma clouds). However, these "ideal" incident beam intensity profiles exhibit very high and narrow peaks illuminating the rim of the target ablator, which may prove difficult to deliver in practice. Use of less "rim-peaked" beam profiles implies a departure from the ideal spherically uniform ablator deposition calculated here, and that implies time-dependant asymmetries would arise.

\section{What is most needed to be learned next:}

Two-D hydro calculations for two-sided polar ion drive are needed to evaluate time-dependant asymmetry amplitudes as functions of the degree of departure of "real" beam profiles from the ideal ones calculated here. Mitigation of resulting hot spot uniformities need to be studied by controlling the time-dependent zoom of various hollow beam profiles, so as to achieve the lowest time-averaged asymmetry. Assuming hot spot ignition needs to be made more robust to work with the residual asymmetries found, then pursue two recovery schemes (a) increase the implosion velocity (at the expense of increased drive energy and lower gain (there is enough gain to "burn" to allow this); or (b) add a powerful late shock, as John Perkins has already found in an ion beam driven example, to "kick-start" the central burn wave.

\section{The prize for success in this effort may be a high performance IFE target more attractive than fast ignition.}

